

\section{Optimal Design of Laminated Composites}

with Focus on Aircraft Structures 
Composition of the graduation committee:

Chairman and Secretary:

Prof. dr. ir. G.P.M.R. Dewulf University of Twente

Supervisors:

Dr. ir. H.J.M. Geijselaers University of Twente

Prof. dr. ir. R. Akkerman University of Twente

Co-supervisor:

Dr. I. Baran

University of Twente

Members:

Prof. dr. C. Bisagni

Delft University of Technology

Prof. dr. ing. A. Schumacher

University of Wuppertal

Prof. dr. ir. D.M. Brouwer

University of Twente

Prof. dr. I. Gibson

University of Twente

ISBN 978-90-365-4736-9

1st printing March 2019

Keywords: Composite panel, Stacking Sequence Table, Blended design, Buckling optimization, Level-set method

This thesis was prepared with $\mathrm{AT}_{\mathrm{E} X} \mathrm{X}$ by the author and printed by Gildeprint, Enschede, from an electronic document.

Copyright (c) 2019 by F. Farzan Nasab, Enschede, The Netherlands.

All rights reserved. No part of this publication may be reproduced by print or photocopy by any other means without the prior written permission from the copyright owner. 


\section{Optimal Design of Laminated Composites with Focus on Aircraft Structures}

\section{DISSERTATION}

to obtain

the degree of doctor at the University of Twente, on the authority of the rector magnificus, prof. dr. T.T.M. Palstra, on account of the decision of the graduation committee, to be publicly defended on Friday the 29th of March 2019 at 12:45 hours

by

Farshad Farzan Nasab

born on the 16th of June 1987

in Tehran, Iran 
This dissertation has been approved by:

Supervisors:

Dr. ir. H.J.M. Geijselaers and

Prof. dr. ir. R. Akkerman

Co-supervisor:

Dr. I. Baran

Advisor:

Prof. dr. ir. A. de Boer 


\section{Summary}

The demand for more efficient commercial aircraft with lower maintenance and operation cost has promoted more extensive use of composite materials for a significant reduction in weight and fuel consumption. Making a structure as light as possible suggests solving an optimization problem with the goal of mass minimization. To avoid failure modes related to composite structures, such as delamination and transverse matrix crack propagation, some design guidelines are commonly suggested by aircraft industries. These guidelines are: symmetry, covering ply, disorientation, percentage rule, balance, and contiguity of the layup.

The manufacturability of the final design with available technologies must be guaranteed. Different regions may be subject to different loads in a large-scale structure. Laminate thickness may vary throughout the structure depending on the distributed loads in an optimized design. Additionally, for large-scale composite structures, such as an aircraft wing or fuselage, stiffeners are added to enhance structural performance in carrying compressive and tensile loads. The stiffeners divide the structure into smaller panels. To ensure manufacturability of a composite structure, it is crucial for the plies to be continuous among adjacent panels while the laminate thickness varies. Continuity of plies in adjacent panels, which is commonly referred to as blending, is a particularly difficult constraint to deal with.

A feasible composite structure has to have sufficient stiffness and strength while complying with the aforementioned design and manufacturability guidelines. The feasibility of a composite structure is evaluated through the constraints added to the optimization problem.

The variables in the design of a composite structure include, but are not limited to, ply stacking sequences and thickness distribution. Depending on the application, it may also be desirable to design the shape of a structure. Holes may be required in the design of parts such as ribs of aircraft wings to reduce the weight. Therefore, the shape and the topology may be additional variables in the design of a composite structure.

Optimization of a composite structure subject to the design, manufacturing, and strength related guidelines is a very challenging problem. Fulfilling the manufacturability guideline in particular has been a major goal in recent studies. This confirms the interest of the experts in the field in the applicability of their proposed methods to real-life engineering problems. However, an investigation among the existing research, 
revealed that these methods require a significantly large number of computations and their inherent complexity makes them inadmissible for application to real structures. This motivated performing the present research with the goal of developing a design tool that can optimize complex fiber-reinforced composite structures in practice.

The present research consists of a design tool for the optimization of variable stiffness composite structures (where fibers are not steered), and a method which is developed mainly for the optimization of an aircraft wing. To optimize a variable stiffness composite structure, the proposed method separates the optimization of stacking sequences from the optimization of the thickness distribution. A set of laminates with optimized stacking sequences with respect to the problem at hand is generated and stored in a reference table known as the Stacking Sequence Table (SST). The laminates in an SST must satisfy the desired laminate design guidelines. As long as the ply stacks in a design are selected from the SST, manufacturability of the final design is guaranteed. Next, a novel level-set gradient based method is introduced for the global optimization of ply drop locations. The proposed method aims at turning the discrete optimization problem associated with the integer number of plies into a continuous problem. This is done through the way the problem is parametrized; the design variables are never rounded in this approach. The level-set function gives the optimum thickness distribution over the structure for a specific SST.

The developed method is verified by its application to the well-known horseshoe panel optimization problem. To investigate the performance of the method in dealing with a real problem, the proposed method is then applied to the layup optimization of a composite skin and rib structure of a wing. Local buckling and allowable strain are considered as the constraint of the problem and a standard finite element package is used to calculate buckling factors.

The structural optimization of an aircraft wing is a highly complex problem. This is due to the large number of variables as well as structural and aerodynamics constraints influencing the design of skins and stiffeners. To make it computationally more efficient, a large problem can be decomposed into several smaller subproblems (subsystems) while preserving the couplings among these subproblems. A special method is subsequently introduced for the optimization of interacting skins and ribs of an aircraft wing box. 


\section{Samenvatting}

Het streven naar efficiëntere commerciële vliegtuigen met lage onderhouds- en gebruikskosten heeft geleid tot een uitgebreider gebruik van vezel versterkte materialen omdat hiermee significant bespaard kan worden op onderhoud, gewicht en brandstofverbruik.

Om falen van composietconstructies, zoals delamineren en scheurgroei in de matrix, te voorkomen, worden een aantal ontwerprichtlijnen aanbevolen door vliegtuigindustrieën. Deze richtlijnen zijn: symmetrie, oriëntatie van de toplaag, beperking van oriëntatie verschillen tussen afzonderlijke lagen, balans en continuiteit van de lay-up.

De maakbaarheid van het eindontwerp met de beschikbare technologieën moet worden gegarandeerd. In grootschalige constructies kunnen verschillende zones onderhevig zijn aan verschillende belastingen. De dikte van het laminaat kan verschillen over de gehele constructie, afhankelijk van de verdeelde belastingen in een geoptimaliseerd ontwerp. Aan grootschalige composietconstructies, zoals een vleugel of romp van een vliegtuig, worden spanten of ribben en verstijvers toegevoegd om de draagkracht voor druk en trek belastingen te verbeteren. De ribben en verstijvers verdelen de constructie in kleinere panelen. Om de maakbaarheid van een composietconstructie te garanderen, moeten de lagen door lopen in aanliggende panelen. Continuïteit van lagen in aanliggende panelen, wat wordt aangeduid met 'blending', is een bijzonder moeilijke eis om te realiseren.

Het zo licht mogelijk maken van een constructie suggereert het oplossen van een optimalisatieprobleem met als doel massaminimalisatie. Een composietconstructie moet voldoende stijfheid en sterkte hebben en voldoen aan de bovengenoemde ontwerpen maakbaarheidsrichtlijnen. De toepasbaarheid van een composietconstructie wordt geëvalueerd aan de hand van de randvoorwaarden die aan het optimalisatieprobleem zijn toegevoegd.

De variabelen in het ontwerp van een composietconstructie omvatten stapelvolgorde en dikteverdeling. Afhankelijk van de toepassing kan het ook wenselijk zijn om de vorm van een constructie te ontwerpen. Bij het ontwerp van bij voorbeeld ribben van vliegtuigvleugels kunnen gaten aangebracht worden om het gewicht te verminderen. Daarom kunnen de vorm en de topologie aanvullende variabelen zijn in het ontwerp van een vezel versterkte constructie. 
Optimalisatie van een composietconstructie onderworpen aan de richtlijnen voor ontwerp, fabricage en sterkte is een zeer uitdagend probleem. Vooral het naleven van de maakbaarheidsrichtlijn is een belangrijk doel geweest in recente onderzoeken. Dit bevestigt de belangstelling van de experts op dit gebied voor de toepasbaarheid van door hun voorgestelde methoden op realistische technische problemen. Een doorlichting van het bestaande onderzoek toonde echter aan dat deze methoden een aanzienlijke hoeveelheid berekeningen vereisen en dat ze in de praktijk niet toepasbaar zijn op werkelijke constructies. Dit motiveerde om het huidige onderzoek uit te voeren met het doel om een ontwerpmethode te ontwikkelen waarmee complexe vezel versterkte constructies in de praktijk kan geoptimaliseerd kunnen worden.

Het resultaat van dit onderzoek is een ontwerptool voor de optimalisatie van composieten met variabele stijfheid (waar vezels niet worden gestuurd) en een methode die voornamelijk is ontwikkeld voor de optimalisatie van een vliegtuigvleugel. Om een samengeste- lde constructie met variabele stijfheid te optimaliseren, scheidt de voorgestelde methode de optimalisatie van stapelvolgorden van de optimalisatie van de dikteverdeling. Een set laminaten met geoptimaliseerde stapelvolgorden, die betrekking hebben op het beschouwde probleem, wordt vervolgens gegenereerd en opgeslagen in een referentietabel genaamd StapelVolgorde Tabel (Stacking Sequence Table, SST). De laminaten in een SST moeten voldoen aan de richtlijnen voor het gewenste laminaatontwerp. Zolang de stapelingen in een ontwerp worden geselecteerd uit de SST, is de maakbaarheid van het uiteindelijke ontwerp gegarandeerd. Vervolgens wordt een nieuwe niveaucontour-gebaseerde methode geïntroduceerd voor de globale optimalisatie van laagdikte verdeling. De voorgestelde methode is gericht op het veranderen van het discrete optimalisatieprobleem, dat is geassocieerd met het gehele aantal lagen, in een continu probleem, de beschrijving van de laagdikte contouren. Dit wordt gedaan door de manier waarop het probleem is geparametriseerd; de ontwerpvariabelen worden in deze benadering nooit afgerond. De niveaufunctie geeft de optimale dikteverdeling over de structuur voor een specifieke SST.

Om de prestaties van de methode in het omgaan met een werkelijk probleem te onderzoeken, wordt de voorgestelde methode toegepast op de lay-upoptimalisatie van een samengestelde huid- en ribstructuur van een vleugel. Lokale knik en toegestane belasting worden beschouwd als de beperking van het probleem en een standaard eindig elementenpakket wordt gebruikt om knikfactoren te berekenen.

De structurele optimalisatie van een vliegtuigvleugel is een zeer complex probleem. Dit komt door het grote aantal variabelen en structurele en aerodynamische beperkingen die van invloed zijn op het ontwerp van huiden en verstevigingsschotten. Om het computationeel efficiënter te maken, kan een groot probleem worden opgedeeld in verschillende kleinere subproblemen (subsystemen), terwijl de koppelingen tussen de niveaus worden behouden. Een speciale methode wordt geïntroduceerd voor de optimalisatie huiden en ribben van een vliegtuigvleugeldoos met in acht name van de interactie tussen beiden. 


\section{Contents}

Summary

Samenvatting vii

I Extended summary 1

1 Introduction 3

1.1 Composite materials . . . . . . . . . . . . . . . . . 3

1.2 Composite materials in aircraft structures . . . . . . . . . . . . 5

1.3 Design of composite structures for aircraft . . . . . . . . . . . . . 7

1.4 Optimization of composite structures . . . . . . . . . . . . . . . . . . . . . .

1.4.1 Heuristic methods . . . . . . . . . . . . . . . . . . 10

1.4 .2 Gradient-based methods . . . . . . . . . . . . . . . 10

1.5 Problem statement and objective of the thesis . . . . . . . . . . . . 11

1.6 Outline of the thesis . . . . . . . . . . . . . . . . . . . . . . . . . . . . .

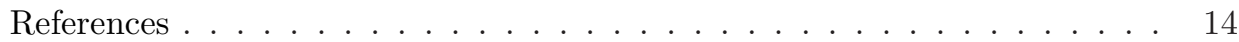

2 Optimization of variable stiffness composite structures $\quad 17$

2.1 Stacking Sequence optimization . . . . . . . . . . . . . . . . 18

2.1.1 Stacking sequence table (SST) . . . . . . . . . . . . . . . . 18

2.1.2 Generating an optimized SST . . . . . . . . . . . . 18

2.1.3 Optimized SST for a stiffened skin . . . . . . . . . . . . 20

2.2 Thickness optimization procedure . . . . . . . . . . . . . . 21

2.2.1 The proposed level-set-based method . . . . . . . . . . 23

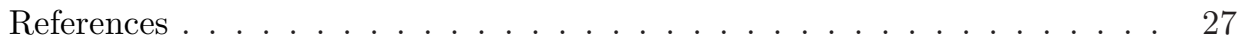

3 Optimization of interacting skins and ribs of a wing box 29

3.1 Rib-level optimization . . . . . . . . . . . . . . . . . 30

3.2 Skin-rib coupling . . . . . . . . . . . . . . . . . . . . . . . . . . . . . . . . .

3.3 Wing-level optimization . . . . . . . . . . . . . . . . . 31

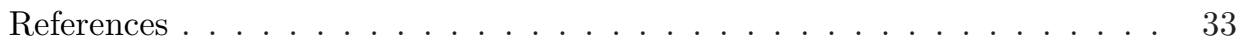


4 Discussion $\quad 35$

4.1 Efficiency of the optimization method . . . . . . . . . . . . . 35

4.1.1 A fully gradient-based method ... . . . . . . . . . . 35

4.1.2 Straightforward in handling the blending guideline . . . . . . . 36

4.1.3 Fast in evaluating the performance of SSTs . . . . . . . . . . 37

4.1.4 Parametrized independently from the ply numbers and possible fiber orientations . . . . . . . . . . . . . . . . . . 37

4.1.5 Flexible in adding extra design variables . . . . . . . . . . . 38

4.1.6 Independent from the (finite element) mesh size of the structure 38

4.1.7 Applicable to the optimization problem of the interacting skins and ribs of an aircraft wing by suggesting a special approach . 38

4.2 Accuracy of the optimization method . . . . . . . . . . . . 39

4.2.1 Global/local optimum . . . . . . . . . . . . . . . . . . 39

4.2.2 Choice of the initial design . . . . . . . . . . . . . . . . . . . . . . 40

4.2.3 Number of panels (or panel-sets) . . . . . . . . . . . . . . . . . 41

4.2.4 Generating the SST . . . . . . . . . . . . . . . 41

4.2.5 Mesh density . . . . . . . . . . . . . . . . . 41

4.2.6 Tracking buckling modes . . . . . . . . . . . . . . . . . . . 42

4.3 Additional remarks . . . . . . . . . . . . . . . . . . . . . . . . . . . . . . . . . .

References.......................... 43

5 Summary, conclusions and recommendations $\quad 45$

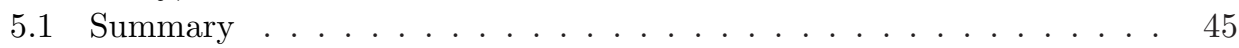

5.2 Conclusions . . . . . . . . . . . . . . . . . . . . . . . 45

5.3 Recommendations ...................... . . 47

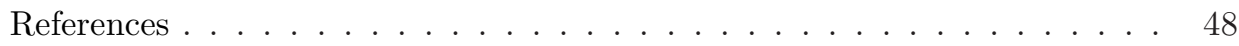

$\begin{array}{lll}\text { II Papers } & 49\end{array}$

\section{Paper A}

A level-set-based strategy for thickness optimization of blended com$\begin{array}{ll}\text { posite structures } & 51\end{array}$

1 Introduction . . . . . . . . . . . . . . . . . . 54

2 Generating a stacking sequence table (SST) . . . . . . . . . . . 56

2.1 Step 1: obtaining the optimized stiffness and thickness distribution (idealized design) . . . . . . . . . . . . . . . . 57

$2.2 \quad$ Step 2: fitting the stacking sequences . . . . . . . . . . . . 58

3 Level-set-based thickness optimization . . . . . . . . . . . . . 60

3.1 The proposed level-set method . . . . . . . . . . . . . . 60

3.2 Optimization objective . . . . . . . . . . . . . . 63

3.3 Constraint definition . . . . . . . . . . . . . . . 63

3.4 Sensitivity analysis . . . . . . . . . . . . . . . 66

3.5 Switching of the mode shapes . . . . . . . . . . . . 66

3.6 Mesh density . . . . . . . . . . . . . . . . 67

4 Results and discussion . . . . . . . . . . . . . . . 67 
4.1 Example 1, Horseshoe Problem . . . . . . . . . . . . . . 67

4.2 Example 2, torsion box skin . . . . . . . . . . . . . . . . . 74

5 Conclusion and outlook . . . . . . . . . . . . . . . . 81

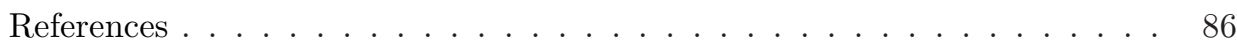

\section{Paper B}

Topology, shape, and thickness optimization of composite structures considering design, manufacturing, and strength related guidelines 91

1 Introduction . . . . . . . . . . . . . . . . . 94

1.1 Gradient based methods . . . . . . . . . . . . . . . . . 94

1.2 Heuristic methods . . . . . . . . . . . . . . . . . 96

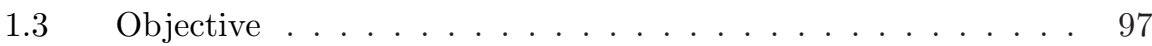

2 Stacking sequence table $(\mathrm{SST}) \ldots \ldots \ldots$. . . . . . . . . . . . 97

3 Thickness, shape, and topology optimization procedure . . . . . . . . . 99

3.1 The proposed level-set-based method . . . . . . . . . . . . 99

3.2 Optimization objective, constraint, and sensitivity analysis . . 102

4 Results and discussion . . . . . . . . . . . . . . . 105

4.1 Example 1, clamped plate . . . . . . . . . . . . . 105

4.2 Example 2, rib optimization . . . . . . . . . . . . . . . 113

5 Summary and conclusion . . . . . . . . . . . . . . 118

References ............................. 124

\section{Paper C}

A gradient-based strategy for the optimization of stiffened composite structures subject to multiple load cases and multiple failure criteria 129

1 Introduction . . . . . . . . . . . . . . . . . . . . 132

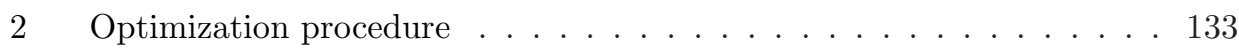

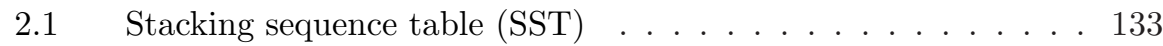

2.2 Thickness optimization . . . . . . . . . . . . . . . . 134

3 Results and discussion . . . . . . . . . . . . . . . . . . 135

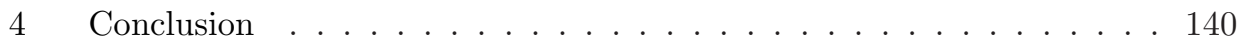

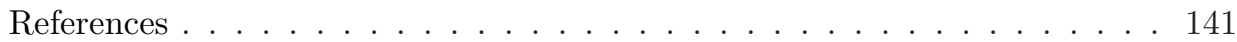

\section{Paper D}

Optimization of the interacting stiffened skins and ribs made of com$\begin{array}{lr}\text { posite materials } & 143\end{array}$

1 Introduction . . . . . . . . . . . . . . . . . . . . . 146

1.1 Strategies for solving the optimization of complex systems . . . 146

1.2 Motivation and aim of the present research . . . . . . . . . . . 147

2 Stacking sequence and thickness optimization . . . . . . . . . . . . 148

3 Quasi-separable decomposition strategy for the skin-rib coupled optimization . . . . . . . . . . . . . . . . 150

3.1 Rib-level optimization . . . . . . . . . . . . . . . . 150

$3.2 \quad$ Skin-rib coupling . . . . . . . . . . . . . . . . 151

3.3 Wing-level optimization . . . . . . . . . . . . . . . 152

4 A model reduction scheme for coupling sensitivities . . . . . . . . . . 153 
5 Application, results, and discussion . . . . . . . . . . . 156

$5.1 \quad$ Finite element model of the wing box . . . . . . . . . . . . 156

5.2 Used SSTs and optimization problem definition . . . . . . . . . 161

5.3 Results and discussion . . . . . . . . . . . . . . . . 165

6 Summary and conclusions . . . . . . . . . . . . . . . . . . . . . . . . . . . . . . . . . . . . . . . . .

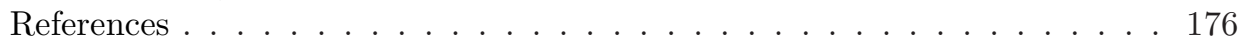

$\begin{array}{llr}\text { III Appendices } & 179\end{array}$

A Constrained steepest-descent method 181

1 Constrained steepest-descent algorithm . . . . . . . . . . . . . . . . 181

1.1 The Quadratic Programming subproblem . . . . . . . . . . . 183

1.2 Step size calculation . . . . . . . . . . . . . 183

B Horizontal stabilizer skin optimization $\quad 185$

$\begin{array}{lr}\text { Nomenclature } & 191\end{array}$

$\begin{array}{ll}\text { Publications } & 193\end{array}$

$\begin{array}{ll}\text { Acknowledgements } & 195\end{array}$ 


\section{Part I}

\section{Extended summary}





\section{1 Introduction}

This thesis concerns the optimization of composite aircraft structures. Composites are increasingly becoming the materials of choice for aircraft structures due to their superior properties. Laminated composites allow for great freedom of design, and both the number of plies and the ply orientation can be chosen to obtain the required properties. Due to the great design freedom offered by these materials, determination of the optimized configuration can be a difficult task. In the following, an introduction to the composite materials and their application in aircraft structures is provided.

\subsection{Composite materials}

Composite materials are formed by combining two or more distinct material phases which together produce enhanced properties that cannot be achieved with any of the constituents alone [1].

Typically, fiber reinforced polymer composites consist of a stiffer reinforcing phase known as Fibers, surrounded by a more compliant Matrix phase. An interphase may exist between the fibers and the matrix [2] to improve bonding between the two. The different phases in a typical composite material are shown in Figure 1.1.

The fibers (usually made of carbon or glass) are the principal load carrying members. The matrix material keeps the fibers together and acts as a load-transfer medium between fibers [1].

In general, the matrix material can be classified into two categories: thermoset and thermoplastic. In recent years, thermoplastic composites have drawn considerable attention thanks to their superior properties. For example, the thermoplastics have a better impact resistance than thermoset composites. This feature is specially important for aircraft structures as they are at high risk of low-velocity impact (such as a bird strike). The other considerable advantage is that thermoplastic composites can be reformed. For example, a pultruded thermoplastic rod can be heated and remolded to have a curvature. The thermoforming capability of thermoplastic composites allows 


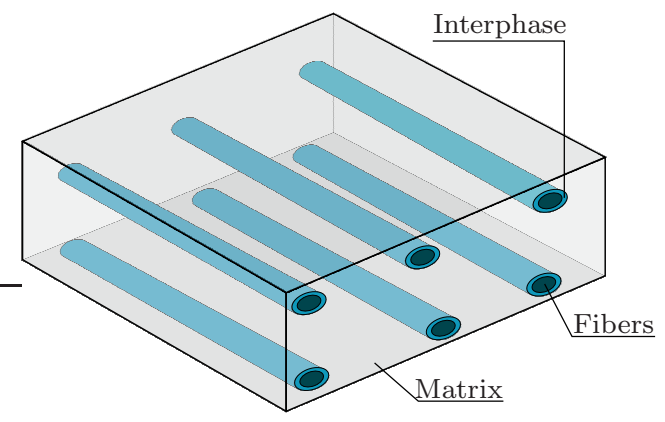

Figure 1.1: Three phases of a composite material.
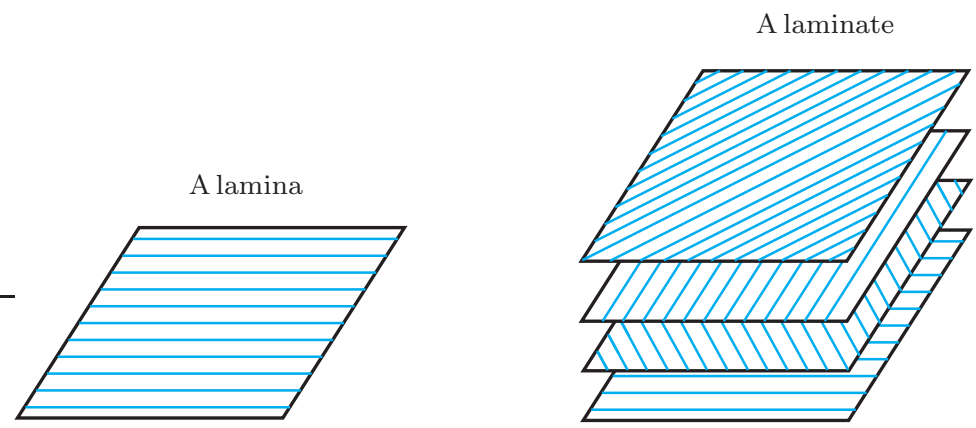

Figure 1.2: A laminate made of several laminae with different fiber orientations.

for the development of various joining techniques such as ultrasonic and induction welding [3]. The inherent recyclability of thermoplastic composites is another advantage of this type of composites over its thermoset counterpart. The promising capabilities of thermoplastic composites in aircraft industry led to the formation of the European consortium TAPAS (Thermoplastic Affordable Primary Aircraft Structure) to further investigate various technologies ranging from welding and pressing to knowledge-based modeling and structural optimization techniques.

Fiber reinforced composite materials are often made in the form of a laminate. A laminate consists of several thin layers called laminae (also known as plies). A lamina contains unidirectional reinforcing fibers. Figure 1.2 shows the build-up of a laminate made of several laminae with different fiber orientations. The stiffness of a lamina in the direction of the fibers $\left(E_{1}\right)$ is usually an order of magnitude larger than that in the transverse direction $\left(\mathrm{E}_{2}\right)$. The strength of a unidirectional lamina depends on the direction of the load with respect to the fiber orientation.

Among various techniques available for the fabrication of composite structures, Automated Fiber Placement (AFP) and Automated Tape Laying (ATL) are the ones that can deliver the high quality required for aircraft structures. A composite structure is made of a series of layers of carbon fiber tapes placed side by side using ATL. The tapes are unrolled from large spools. Figure 1.3 shows a machine supplied with spools 


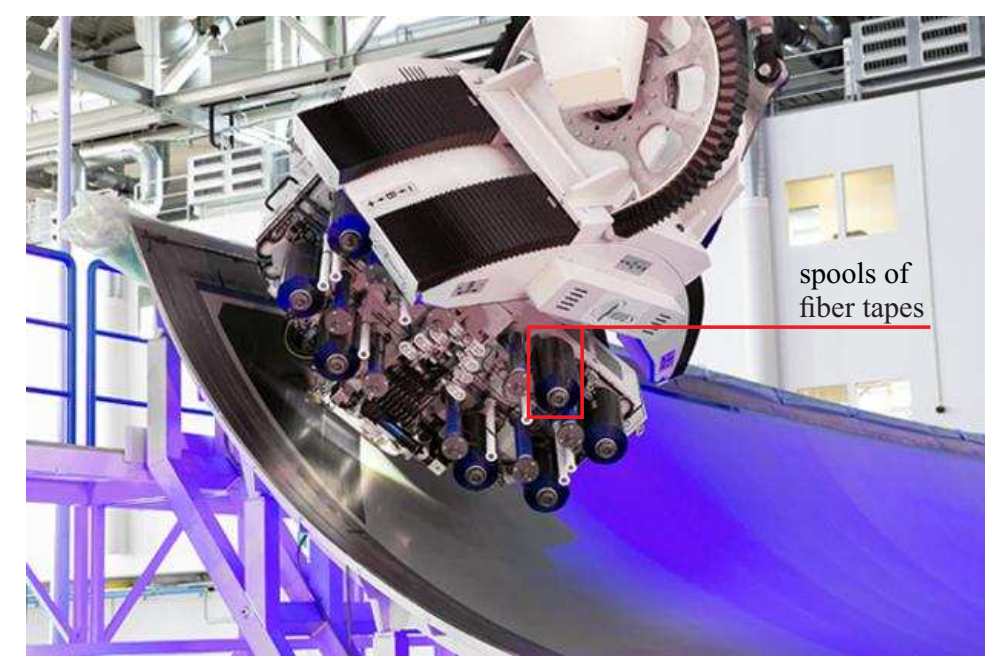

Figure 1.3: A machine supplied with spools of (carbon) fiber tapes.

of tapes.

To manufacture the skin of an aircraft wing, for example, the tapes are laid side by side from the root to the tip of the wing. When the required area has been covered with a layer with a specific fiber orientation, the next layer will be placed. The machine has the ability to place the fiber tapes at different angles. The number of layers and the sequence of the fiber orientations determine the stiffness and the strength of the structure. Figure 1.4 shows the ATL machine fabricating the wing skin of the Airbus A350.

\subsection{Composite materials in aircraft structures}

The demand for a significant reduction in weight and fuel consumption fosters a more extensive use of composite materials. Moreover, the application of composite materials improves the fatigue life and corrosion resistance in comparison with aluminum $[4]$.

The first significant application of composite materials in primary structures of a civil aircraft appeared in the 1980s with the Boeing 737, where the horizontal stabilizer was made out of carbon fiber composite. This was followed by a larger-scale application on the Airbus A320, where the horizontal and vertical stabilizer as well as the control surfaces were made of carbon fiber and glass fiber reinforced composite material. The application of composite materials in the fuselage and wing was performed in the Boeing 787 for the first time and then in the Airbus A350. Figure 1.5 shows the forward fuselage of Boeing 787, and Figure 1.6 shows the wing skin of the Airbus A350, manufactured with fiber reinforced composite materials. 


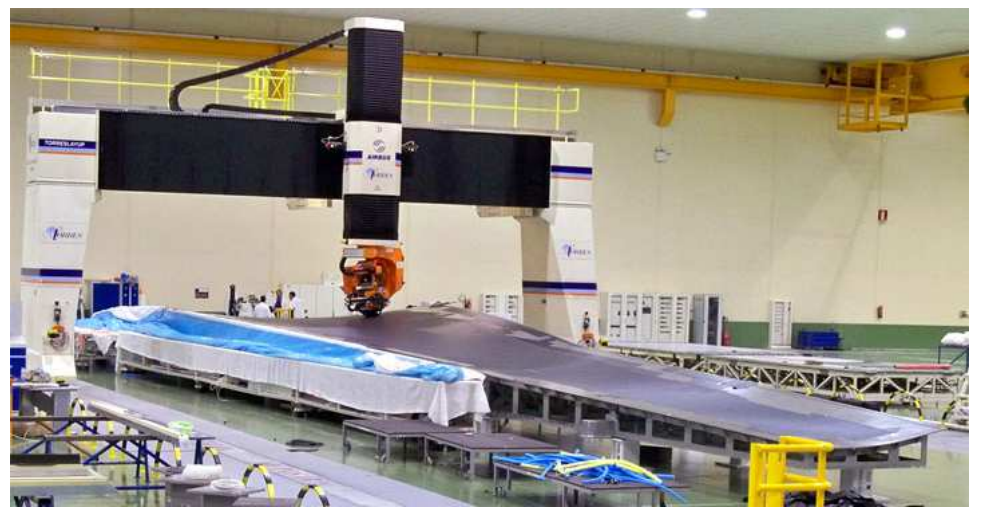

Figure 1.4: The ATL machine manufacturing the wing skin of the Airbus A350. Source: Airbus.

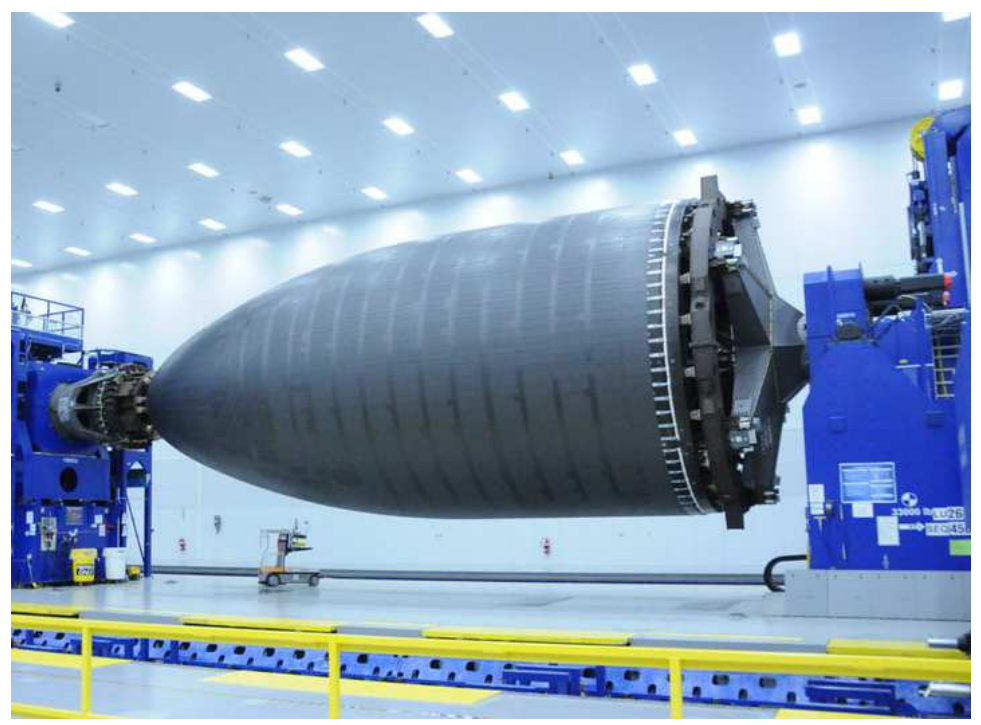

Figure 1.5: The forward fuselage of the Boeing 787 is made of composite materials (using AFP method). Photo by Daniel Terdiman. 


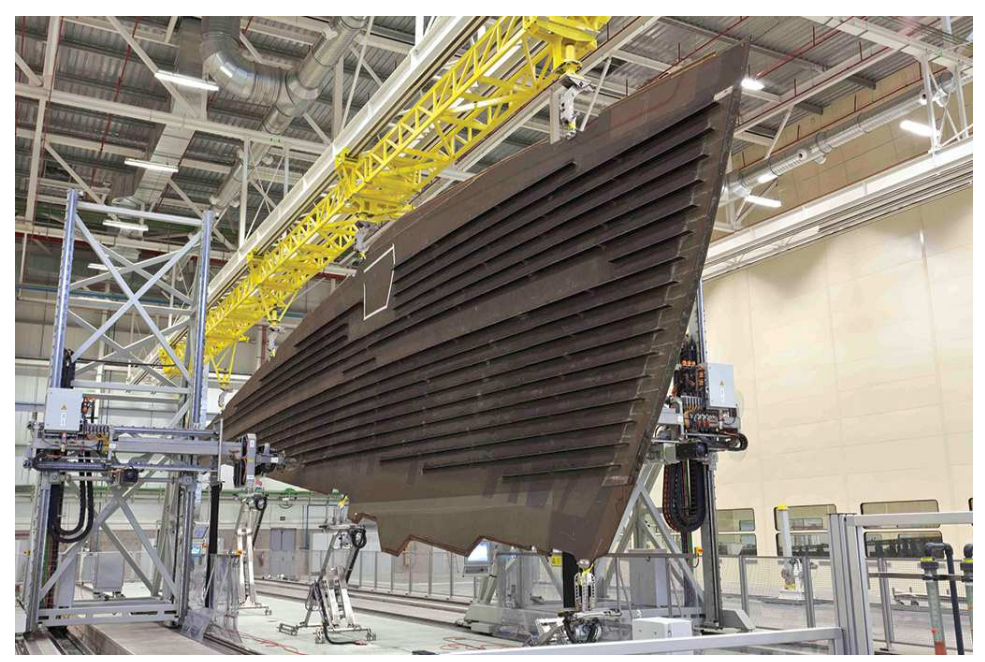

Figure 1.6: The wing skin of the Airbus A350 made of composite materials. Source: Airbus.

This wide application of composite materials in an aircraft structure (about $50 \%$ of the entire structure) provided the required efficiency improvement in terms of reduced operation and maintenance cost [4]. Figure 1.7 shows the history of the amount of composites (by weight) used in various civil aircraft models [4].

As seen in Figure 1.7, the composite material application started slowly and was followed by a rapid growth thanks to increased knowledge of the manufacturing techniques to fabricate reliable composite structures more efficiently.

\subsection{Design of composite structures for aircraft}

The major reason behind the application of composite materials in aircraft structures is to make the structure lighter. To avoid failure modes such as delamination and transverse matrix crack propagation, some design guidelines are commonly suggested. These guidelines, comprehensively discussed in $[5,6]$, are:

- Symmetry, the laminate should be symmetric with respect to its center line.

Reasoning: For a symmetric laminate, the extension/bending coupling is zero. The extension/bending coupling of unsymmetric laminates can reduce buckling loads [5].

- Covering plies, the outermost ply has to have the orientation of either $+45^{\circ}$ or $-45^{\circ}$.

Reasoning: The primary load carrying plies have to be shielded from the exposed surfaces. 


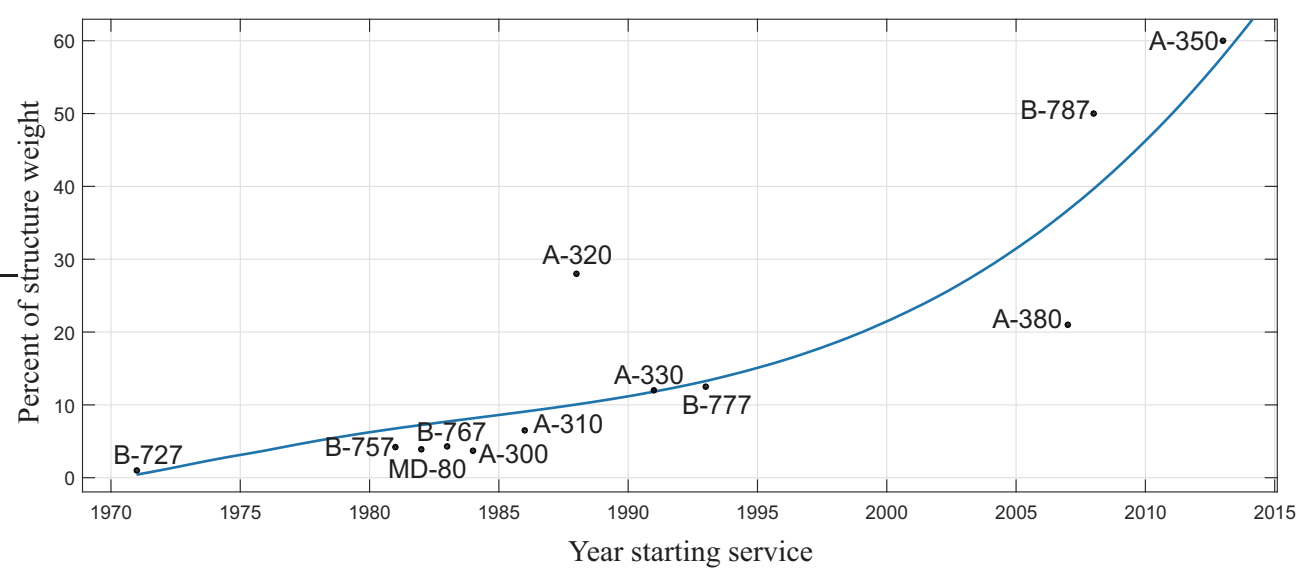

Figure 1.7: Application of composite materials in civil aircraft structures.

- Disorientation, the maximum orientation difference of two adjacent plies is $45^{\circ}$. Reasoning: This guideline aims at reducing the inter-laminar stresses at the edges, thus avoiding ply delamination.

- Percentage rule, the number of plies of a certain orientation has to be at least $10 \%$ of the total number of plies in a laminate.

Reasoning: This guideline guarantees a minimum strength for a laminate in different directions.

- Balance, the total number of plies with $+45^{\circ}$ orientation in a laminate is equal to the total number of plies with $-45^{\circ}$ orientation.

Reasoning: The purpose of using this guideline is to avoid shear/extension coupling and dimensional stability problem (e.g. warpage which affects component manufacturing tolerances [5]). Note that the shear/extension coupling may be desired for some applications such as aeroelastic tailoring.

- Contiguity, not more than four successive plies with the same orientation are allowed to stack together.

Reasoning: This guideline is employed to avoid transverse matrix crack propagation. If several plies with the same orientation are stacked together, a matrix crack forming in one of them can grow easily in the matrix and extend from one side of the identical ply stack to the other without being blocked [4].

In addition, the manufacturability of the final design with available technologies must be guaranteed. Different regions may be subject to different loads in a large-scale structure. A laminate thickness may vary over the structure depending on the distributed loads. Also, for large scale composite structures, such as an aircraft wing or fuselage, stiffeners are added to enhance structural performance in carrying compressive and tensile loads. The stiffeners divide the structure into smaller panels. 


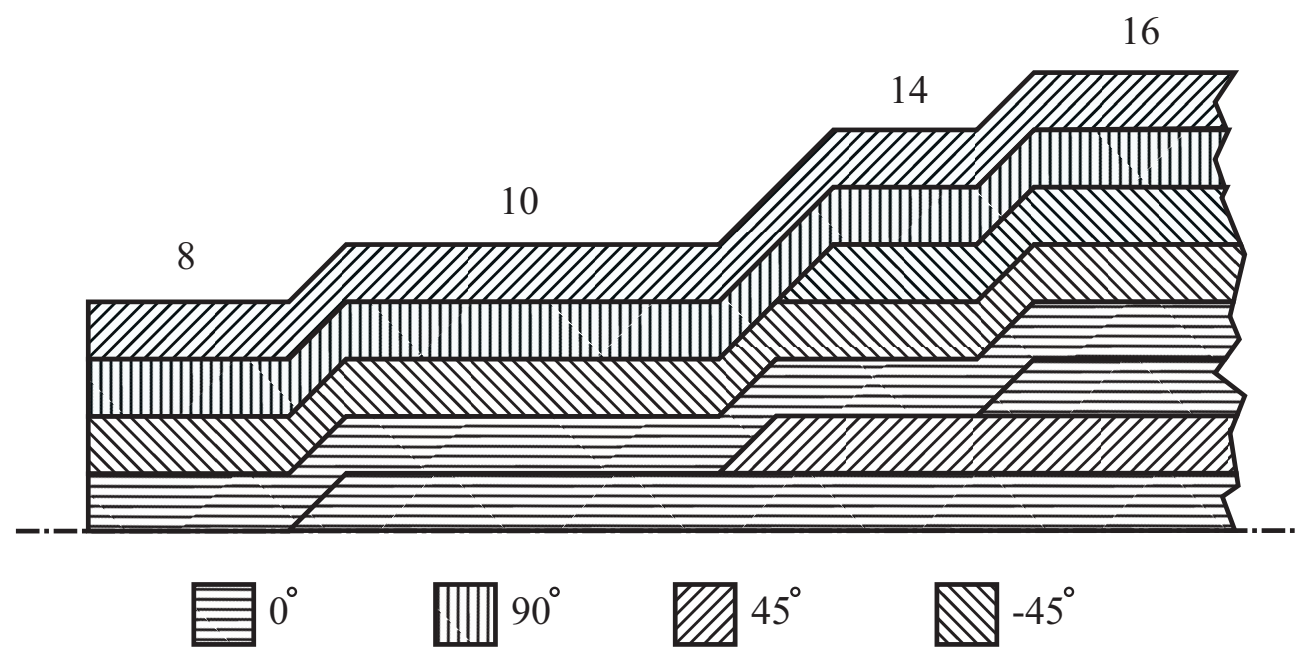

Figure 1.8: A blended structure. Due to symmetry, the plies in half laminates are shown.

Designing panels individually using local loads results in designs with significant manufacturing difficulties. The reason is that the resulting stacking sequences (placement order of the reinforcing fiber orientations) of laminates in adjacent panels may differ considerably [7]. To ensure manufacturability of a composite structure, it is crucial for the plies to be continuous among adjacent panels while the laminate thickness varies. Continuity of plies in adjacent panels, which is commonly referred to as blending [8], is a particularly difficult requirement to deal with [9]. Figure 1.8 shows schematically a blended structure where the thickness varies.

\subsection{Optimization of composite structures}

Achieving a light design requires solving an optimization problem with the goal of mass minimization. A feasible composite structure has to have sufficient stiffness and strength while complying with the aforementioned design and manufacturability guidelines. The feasibility of a composite structure is evaluated through the constraints added to the optimization problem. It is the task of an optimization method to provide a design with sufficient strength and stiffness for the structure with a minimal mass.

The variables in the optimization of a composite structure include, but are not limited to, ply stacking sequences and thickness distribution. Depending on the application, it may also be desirable to optimize the shape of a structure. Holes may be required in the design of, for example, ribs of aircraft wings to reduce the weight. Therefore, the shape and the topology may be additional variables in the design.

Optimization of a composite structure with the earlier mentioned variables subject 
to the design, manufacturing, and strength related guidelines is a very challenging problem [9-11].

The majority of the currently existing methods concerning the optimization of composite structures considering (some of) the aforementioned design and manufacturing guidelines as well as their strength and stiffness, can be divided into two categories, namely heuristic and gradient-based methods.

\subsubsection{Heuristic methods}

The first category involves methods that are based on heuristics such as evolutionary algorithms [12]. The most commonly used algorithm from this category is a Genetic Algorithm (GA). A GA is a search technique well suited for optimization problems with discrete design variables $[12,13]$. Due to the discrete nature of the design variables (number of plies, and discrete fiber angles) involved in the optimization of composite structures, GAs are popular and widely used to find an optimized design for these structures.

In practice, finite element analysis is frequently required to evaluate optimization objective and constraints. A typical finite element model of a complex structure such as an aircraft introduces a large number of degrees of freedom. This results in an expensive analysis even for simplified cases (e.g. simplified geometries or fewer load cases).

A GA requires a large number of analyses. When these analyses involve finite element solutions, this makes a GA-based method computationally very expensive [11] and, therefore, impractical for application to many real engineering problems.

\subsubsection{Gradient-based methods}

The second category includes methods that employ a gradient-based optimization algorithm. These algorithms require the derivatives of the objective and constraint functions with respect to the design variables of the problem. The derivatives are used to find a descent direction to iteratively improve an initial design towards an optimal design. In a gradient-based method, the computation cost is directly related to the number of design variables in the problem.

Various gradient-based optimization algorithms exist and are successfully applied to the design optimization of engineering problems. Although these algorithms require extra computation to derive the derivatives of the functions, their very fast convergence rate makes these algorithms considerably cheaper than the heuristic algorithms. A drawback of the gradient-based algorithms is that the convergence to a global optimum is not guaranteed.

In Paper A and Paper B, several GA-based and gradient-based methods are reviewed and their capabilities and deficiencies are discussed. 


\subsection{Problem statement and objective of the thesis}

A large number of studies in the field of optimization of composite structures had been performed before conducting the present research. In this section, the drawbacks in the existing methods that motivated performing the present research are explained.

Fulfilling the manufacturability guideline in particular, has been a major goal in recent studies (see e.g. [7, 14-17]). This confirms the interest of the experts in the field in the applicability of their proposed methods to real-life engineering problems.

However, an investigation among the existing research revealed that the available methods were applied primarily to excessively simplified examples. These simplifications concern both the mechanical aspects of the problem and the geometry of the model. Ignoring the load redistribution when changing the design of a structure or ignoring some of the required design guidelines which may lead to the failure of the structure are examples in this regard.

One may argue that the results presented in literature are just for the sake of demonstration and the methods behind them may be applicable to real engineering problems too. However, this is not generally the case as the computation cost of solving even those simplified problems is so high that the application of those methods to a realistic problem seems impossible.

As mentioned earlier, a GA is popular because it can be conveniently used for the optimization of composite structures. As will be discussed in Chapter 4, using a GAbased method, a large number of function evaluation is required before an optimized result is obtained for a problem where both geometry and mechanical aspects are extremely simplified (Horseshoe Problem studied in [7, 10, 17-22].) This raises the following question:

Can we really use a GA-based method for the optimization of a complex structure made of fiber reinforced polymer composites?

Gradient-based methods are preferred over the GA-based methods for the sake of computation efficiency. However, a gradient-based method is not necessarily efficient. For example, the well-known density-based method $[23,24]$ and its extension to account for the optimization of composite structures may become very expensive when applied to a real problem (details in this regard can be found in Paper B).

The large number of computations and the inherent complexity involved in the existing methods make them inadmissible for application to real structures and thus make the attempt to produce a manufacturable design meaningless.

In brief, the existing methods were incapable of optimizing a complex composite structure for practical applications where the required design, manufacturing, and strength related guidelines needed to be fulfilled. This was the motivation behind conducting the present research.

Following the research motivation, the goal of this $\mathrm{PhD}$ study is:

To develop a method that can optimize complex fiber-reinforced laminated composite structures in practice. 
Ideally, this optimization method has the following key characteristics:

- Fulfills the suggested rules relating to the design of a composite structure.

- Provides a manufacturable design.

- Can be easily adapted to any structural finite element package. This is required when functions' gradients cannot be easily obtained analytically.

- Is efficient in terms of computational cost.

Although the target design method has general application, particular attention is paid to design of aircraft structures.

\subsection{Outline of the thesis}

This thesis is divided into three parts: in Part I, an introduction is followed by an overview of the theories related to the developed optimization method as well as a discussion and conclusions. In Part II, four articles are appended to provide detailed information on the optimization method, validations, and examples. Figure 1.9 shows the aim of each article and the relation between the articles and the developed optimization method. Part III includes two appendices. The first one describes the used mathematical optimization algorithm and the second one introduces a special reference for the stacking sequences designed for the optimization of the skin of the horizontal stabilizer of an aircraft.

In Part I, Chapter 2 describes a method for the optimization of variable stiffness composite structures. First a Stacking Sequence Table (SST) and the method to optimize it are introduced in Section 2.1. The stacking sequence optimization part is concluded by presentation of the results related to optimization of an SST for the skins of a wing box. In Section 2.2, the developed level-set-based method for the optimization of the thickness distribution using an SST is explained. The application of the proposed method to shape and topology optimization of composite structures is discussed.

A method for the optimization of interacting skins and ribs of a wing box is introduced in Chapter 3. The coupling terms that relate the optimization of skins to the optimization of ribs are explained. A scheme based on Principal Component Analysis (PCA) to reduce the computation cost relating to the calculation of coupling sensitivities is presented in this chapter.

Chapter 4 provides an extensive discussion on the proposed optimization method. The accuracy and the efficiency of the proposed design method are discussed.

The main conclusions of the present research and some recommendations for future studies are presented in Chapter 5. 


\section{Papers in Part II}

\section{Aim of the paper}

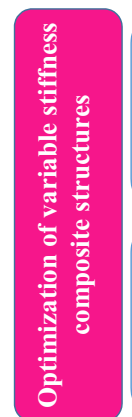

\section{Paper A}

A level-set-based strategy for thickness optimization of blended composite structures

\section{Paper B}

Topology, shape, and thickness optimization of composite structures considering design, manufacturing, and strength related guidelines
- Introduces the procedure for the optimization of stacking sequences.

- Introduces the level-set based thickness optimization method.

- Extends the level-set based thickness optimization to account for topology and shape optimization.

\section{Paper C}

A gradient-based strategy for the optimization of stiffened composite structures subject to multiple load cases and multiple failure criteria

\section{Paper D}

Optimization of the interacting stiffened skins and ribs made of composite materials
- Introduces a decomposition technique for the optimization of interacting skins and ribs of a wing.

- Introduces a reduction scheme for the calculation of the sensitivities.

Figure 1.9: Schematic overview of the articles in Part II. 


\section{References}

[1] J. N. Reddy. Mechanics of laminated composite plates : theory and analysis. Boca Raton : CRC Press, 1997.

[2] S. T. IJsselmuiden. Optimal Design of Variable Stiffness Composite Structures using Lamination Parameters. PhD thesis, TU Delft, 2011.

[3] F. C. Campbell. Manufacturing Processes for Advanced Composites. Elsevier Science, 2004.

[4] C. Kassapoglou. Design and Analysis of Composite Structures: With Applications to Aerospace Structures. John Wiley and Sons, second edition, 2013.

[5] Military Handbook - MIL-HDBK-17-3F: Composite Materials Handbook, Volume 3 - Polymer Matrix Composites Materials Usage, Design, and Analysis. U.S. Department of Defense, 2002.

[6] J. A. Bailie, R. P. Ley, and A. Pasricha. A summary and review of composite laminate design guidelines. Technical report NASA, NAS1-1934\%. Northrop Grumman-Military Aircraft Systems Division, 1997.

[7] D. B. Adams, L. T. Watson, Z. Gürdal, and C. M. Anderson-Cook. Genetic algorithm optimization and blending of composite laminates by locally reducing laminate thickness. Advances in Engineering Software, 35(1):35-43, 2004.

[8] H. Ghiasi, K. Fayazbakhsh, D. Pasini, and L. Lessard. Optimum stacking sequence design of composite materials part 2: Variable stiffness design. Composite Structures, 93(1):1 - 13, 2010.

[9] G. Allaire and G. Delgado. Stacking sequence and shape optimization of laminated composite plates via a level-set method. Journal of the Mechanics and Physics of Solids, 97:168 - 196, 2016.

[10] J. Yang, B. Song, X. Zhong, and P. Jin. Optimal design of blended composite laminate structures using ply drop sequence. Composite Structures, 135:30 - 37, 2016.

[11] Y. M. Meddaikar, F. X. Irisarri, and M. M. Abdalla. Laminate optimization of blended composite structures using a modified Shepard's method and stacking sequence tables. Structural and Multidisciplinary Optimization, 55(2):535-546, 2017.

[12] I. Baran, C. C. Tutum, and J. H. Hattel. Optimization of the thermosetting pultrusion process by using hybrid and mixed integer genetic algorithms. Applied Composite Materials, 20(4):449-463, Aug 2013.

[13] C. C. Tutum, I. Baran, and K. Deb. Optimum design of pultrusion process via evolutionary multi-objective optimization. The International Journal of Advanced Manufacturing Technology, 72(9):1205-1217, Jun 2014. 
[14] D. B. Adams, L. T. Watson, and Z. Gürdal. Optimization and blending of composite laminates using genetic algorithms with migration. Mechanics of Advanced Materials and Structures, 10:183-203, 2003.

[15] B. P. Kristinsdottir, Z. B. Zabinsky, M. E. Tuttle, and S. Neogi. Optimal design of large composite panels with varying loads. Composite Structures, 51:93-102, 2001.

[16] M. T. McMahon and L. T. Watson. A distributed genetic algorithm with migration for the design of composite laminate structures. Parallel Algorithms and Applications, 14:329-362, 2000.

[17] G. Soremekun, Z. Gürdal, C. Kassapoglou, and D. Toni. Stacking sequence blending of multiple composite laminates using genetic algorithms. Composite Structures, 56(1):53-62, 2002.

[18] F. X. Irisarri, A. Lasseigne, F. H. Leroy, and R. Le Riche. Optimal design of laminated composite structures with ply drops using stacking sequence tables. Composite Structures, 107:559-569, 2014.

[19] S. T. IJsselmuiden, M. M. Abdalla, O. Seresta, and Z. Gürdal. Multi-step blended stacking sequence design of panel assemblies with buckling constraints. Composites Part B: Engineering, 40(4):329 - 336, 2009.

[20] O. Seresta, M. M. Abdalla, and Z. Gürdal. A genetic algorithm based blending scheme for design of multiple composite laminates. In Collection of Technical Papers - AIAA/ASME/ASCE/AHS/ASC Structures, Structural Dynamics and Materials Conference, 2009.

[21] P. Jin, X. Zhong, J. Yang, and Z. Sun. Blending design of composite panels with lamination parameters. The Aeronautical Journal, 120(1233):1710-1725, 2016.

[22] P. Jin, Y. Wang, X. Zhong, J. Yang, and Z. Sun. Blending design of composite laminated structure with panel permutation sequence. Aeronautical Journal, 122(1248):333-347, 2018. cited By 0.

[23] M. P. Bendsøe and N. Kikuchi. Generating optimal topologies in structural design using a homogenization method. Computer Methods in Applied Mechanics and Engineering, 71(2):197 - 224, 1988.

[24] M. P. Bendsøe. Optimal shape design as a material distribution problem. Structural optimization, 1(4):193-202, Dec 1989. 



\section{Optimization of variable stiffness composite structures}

To optimize a variable stiffness composite structure, the proposed method separates the optimization of stacking sequences from the optimization of the thickness distribution. The stacking sequences are optimized based on an estimation of the optimized stiffness and thickness distribution over the structure. A set of laminates with optimized stacking sequences with respect to the problem at hand is generated and stored in a reference table known as Stacking Sequence Table (SST). The laminates in an SST must satisfy the desired laminated design guidelines. As long as the ply stacks in a design are selected from the same SST, manufacturability of the final design is guaranteed.

Next, a level-set gradient based method is introduced for the global optimization of ply drop locations. A single function determines the span of multiple levels where each level represents a ply in a stiffened composite skin. This stands in contrast to the studies where multiple level-set functions are used to represent multiple material phases $[1,2]$. The proposed method aims at turning the discrete optimization problem associated with the integer number of plies into a continuous problem. This is done through the way the problem is parametrized; the design variables are never rounded in this approach. The level-set function gives the optimum thickness distribution over the structure for a specific SST.

The developed method is verified by its application to the well-known Horseshoe panel optimization problem studied in [3-9] (see Paper A). To investigate the performance of the method in dealing with a real problem, the proposed method is then applied to the layup optimization of a composite skin and rib structure of a wing (see Paper A and Paper B). Local buckling is considered as the constraint of the problem and a standard finite element package is used to calculate buckling factors. 


\subsection{Stacking Sequence optimization}

\subsubsection{Stacking sequence table (SST)}

An SST is a reference table for the stacking sequences of laminates with different thicknesses. Each column of the SST represents the stacking sequence of a certain number of plies. First introduced by Carpentier et al. [10], the SST has been shown to be a convenient aid in obtaining a globally blended design. To guarantee the blending of a design, a thicker stacking sequence can be obtained only by adding plies to a thinner one [3]. To keep the design blended during the optimization process, the laminates across the structure are only allowed to be selected from the same SST. Industrial requirements demand that the ply angles should be selected from a limited set of angles $[6,11]$. In the present study, the ply orientations are limited to the set $\left\{0^{\circ}, \pm 45^{\circ}\right.$, and $\left.90^{\circ}\right\}[11,12]$. Every laminate in an SST may be required to satisfy a number of strength related guidelines. As described in Section 1.3, in this research, the following conventional guidelines proposed and applied in [6, 11-14], are imposed in an SST: symmetry, covering ply, disorientation, percentage rule, balance, and contiguity.

In general, imposing the aforementioned guidelines has a large influence on the complexity, computation cost, and quality of the optimum design (see e.g. $[15,16]$ ). Here, a method for generating an SST is proposed that can be simply adapted to ignore or relax any of the aforementioned strength related guidelines.

\subsubsection{Generating an optimized SST}

Generating an SST based only on the mentioned composite design guidelines results in several SSTs. Although these SSTs are all valid in terms of design rule fulfillment, they perform differently when used for the design of a structure. The SST that shows the best performance in terms of the quality of optimum design is called the optimized SST. In Paper A, a two-step procedure is introduced to obtain a unique optimized SST.

\section{Step 1: obtain the optimized stiffness and thickness distribution (idealized design)}

An optimized SST must be generated based on the applied loads, the constraints of the problem, and the objective function (e.g. mass, compliance, etc.). Thus to generate an SST, first the optimized stiffness and thickness distribution are estimated $[11,14,17-$ 19]. To this end, lamination parameters [20, 21], polar parameters $[18,19]$, or the smeared stiffness method [11] can be used. The smeared stiffness method requires fewest parameters and is computationally the least expensive. Therefore, it has been selected to obtain an estimate of the optimized stiffness and thickness distribution (idealized design [14]).

The smeared stiffness method estimates the extensional (A) and the bending (D) stiffness matrices without defining the stacking sequence of a laminate. This is achieved by assuming a homogeneous section for the layups [11]. 
Liu et al. [11] proposed solving the following optimization problem to obtain an estimation about thickness and stiffness distribution over the structure:

$$
\min f=\sum_{j=1}^{N_{\mathrm{p}}}\left(n_{0}^{j}+n_{45}^{j}+n_{90}^{j}\right) S_{j} t
$$

where $N_{\mathrm{p}}$ is the total number of panels in the entire structure, $S_{j}$ is the area of panel $j$, and $t$ is the ply thickness. The number of plies of each orientation in each panel (or a set of panels), $n_{0}^{j}, n_{45}^{j}, n_{90}^{j}, j=1, \ldots, N_{\mathrm{p}}$ are the design variables of this optimization problem. Due to the assumed balance guideline, the number of $+45^{\circ}$ plies has to be equal to the number of $-45^{\circ}$ plies. Therefore, $n_{45}^{j}$ is defined as the sum of $+45^{\circ}$ and $-45^{\circ}$ plies.

Here, constraints of the optimization problem in Equation (2.1) are defined as follows:

$$
\begin{array}{cl}
g_{i} \leq 0 & i=1 \text { to } N_{\mathrm{c}} \\
n_{0}^{j} / N^{j} \geq 0.1 & j=1 \text { to } N_{\mathrm{p}} \\
n_{45}^{j} / N^{j} \geq 0.2 & j=1 \text { to } N_{\mathrm{p}} \\
n_{90}^{j} / N^{j} \geq 0.1 & j=1 \text { to } N_{\mathrm{p}}
\end{array}
$$

where $g_{i}$ is a constraint related to the mechanical response of the structure (such as buckling, or strain), $N_{\text {c }}$ represents the number of required constraints, and $N^{j}=$ $n_{0}^{j}+n_{45}^{j}+n_{90}^{j}$. As can be seen in Equation (2.2), it is required that the percentage of the plies of each orientation is $\geq 10 \%$ in each panel.

Solving the optimization problem defined in Equation (2.1) gives an estimation of the 'idealized' stiffness and thickness distribution. The output of this step is a table called SST-data. Each thickness value in an SST-data table appears once and a unique vector of stiffness properties is assigned to every (rounded to the nearest integer) thickness value (see Paper A).

\section{Step 2: fit the stacking sequences}

In the second step, the SST-data table obtained in step 1 is used to generate stacking sequences of laminates with different thickness values. In the procedure of generating the stacking sequences, all required laminate design guidelines have to be satisfied.

All valid laminates (laminates that satisfy the strength related guidelines) with the thickness equal to that of the thinnest laminate obtained in the step 1 are generated. Among the set of the valid thinnest laminates, the one which has the closest stiffness values to those estimated for the same thickness laminate in step 1 , is selected.

To generate a thicker laminate, a ply (or two plies if the fiber orientations are $-45^{\circ}$ and $+45^{\circ}$ ) has to be added to a thinner laminate. All valid laminates, according to the required guidelines, are generated. From the set of the newly built laminates the one with stiffness values closest to those of the laminate with the same thickness in the SST-data table is selected. This procedure continues until a laminate with the thickness equal to that of the thickest laminate in the SST-data table is reached. Detailed information on generating an SST can be found in Paper A. 


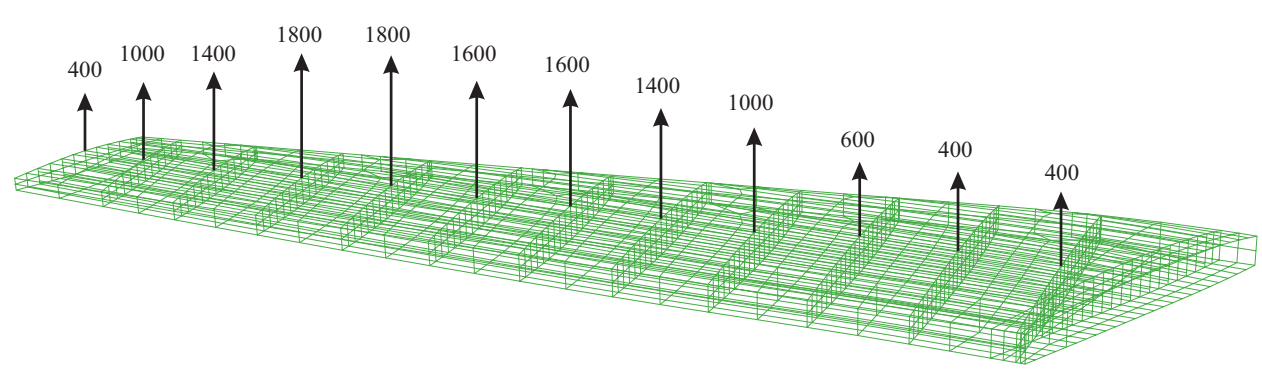

Figure 2.1: The wing box subjected to an upward bending load case. The loads are given in $[\mathrm{N}]$.

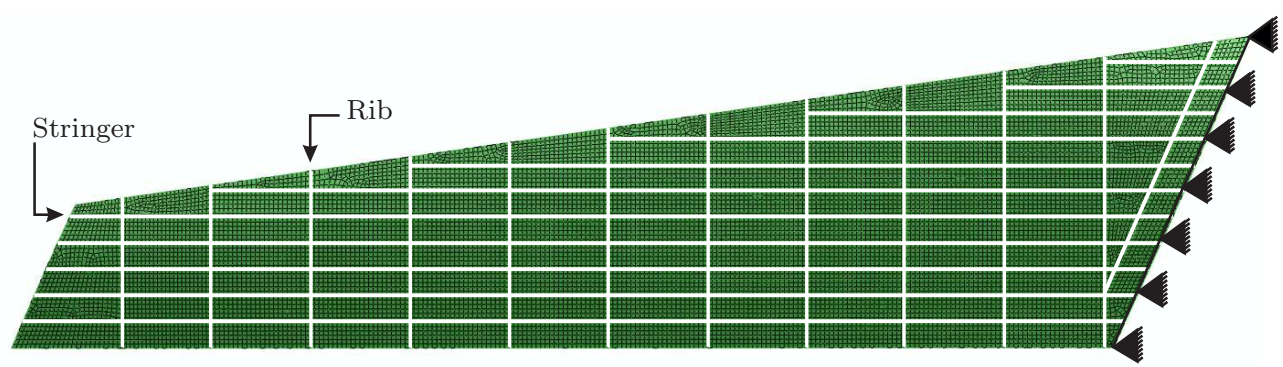

Figure 2.2: The stiffened top skin and the boundary condition applied to it. The skin is simply supported on the right edge and the out of plane translational degree of freedom for all edges and stiffeners (stringers, spars, and ribs) is set to be zero.

\subsubsection{Optimized SST for a stiffened skin}

The two-step method proposed for generating an SST is employed for the stacking sequence optimization of the top and the bottom skin of a wing box. The wing box is subject to an upward bending load case as shown in Figure 2.1. The free body diagram loads of the top and the bottom skin are derived from the wing box model. Figure 2.2 shows the stiffened top skin and the boundary condition applied to it (as the geometry of both skins is almost similar and the boundary conditions are identical for both skins, only the top skin is shown). The top skin is subject to compressive loads and the bottom skin is subject to tensile loads.

To obtain the idealized stiffness and thickness distribution, the optimization problem defined in Equation (2.1) is solved. Buckling and strain are considered as the constraint for the top and the bottom skin, respectively. The stiffeners divide the skin shown in Figure 2.2 into 99 panels. Assigning a set of design variables to each panel results in a large number of variables which makes it expensive to solve the optimization problem defined in Equation (2.1). As the purpose of solving this optimization problem is to only obtain an idealized design, a cheaper problem can be solved instead. Here, the 99 panels are divided into 8 larger sets of panels (called panel-sets). Figure 2.3 shows the division of the skin into the panel-sets. Three design variables 


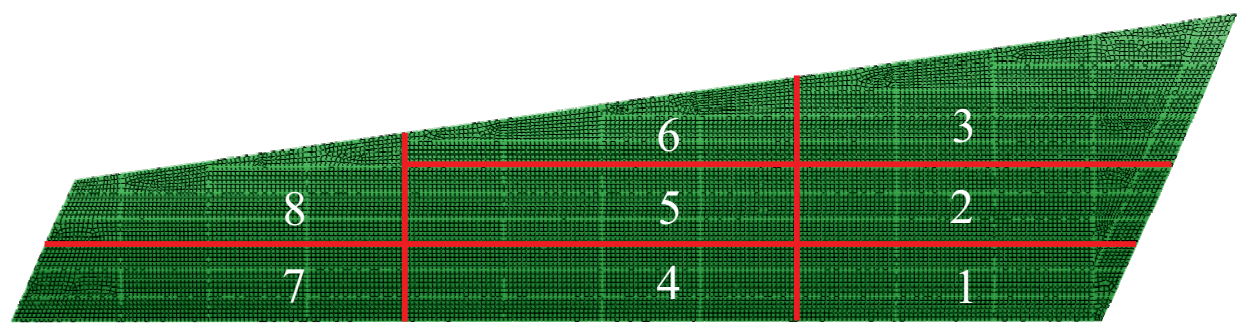

Figure 2.3: The division of the skin into eight panel-sets.

Table 2.1: The result of the optimization problem defined in Equation (2.1) for the top skin.

\begin{tabular}{ccccc}
\hline Panel-set & $n_{0}$ & $n_{45}$ & $n_{90}$ & $\begin{array}{c}\text { Idealized number of plies (rounded) } \\
2\left(n_{0}+n_{45}+n_{90}\right)\end{array}$ \\
\hline 1 & 1.1 & 4.7 & 1 & 14 \\
2 & 2 & 10.3 & 6.4 & 37 \\
3 & 3.1 & 16.7 & 8.2 & 56 \\
4 & 1 & 2 & 1 & 8 \\
5 & 1 & 2 & 1 & 19 \\
6 & 1 & 6.3 & 2 & 8 \\
7 & 1 & 2 & 1 & 8 \\
8 & 1 & 2 & 1 & \\
\hline
\end{tabular}

(corresponding to $n_{0}^{j}, n_{45}^{j}, n_{90}^{j}$ ) are assigned to each panel-set. The result of the optimization problem is shown in Table 2.1 and Table 2.2 for the top and the bottom skin, respectively.

As seen in Table 2.1, for panel-sets with larger number of plies, $n_{45}$ obtains the highest value among the design variables. The reason is that the top skin is subject to buckling constraint. To improve the buckling resistance of a plate, the torsional rigidity of the plate has to be improved [22]. For a laminate, the torsional rigidity is related to $D_{66}$ which can be enhanced by adding more $\pm 45^{\circ}$ plies.

In Table 2.2, panel-sets 1 and 4 have a relatively large number of plies. In these panel-sets, $n_{0}$ obtains the highest value among the other design variables. The reason is that the bottom skin is under tensile load. To improve the tensile strength of the skin, plies in the direction of the tensile load (here, $0^{\circ}$ plies) are required.

Using the aforementioned results, the SSTs shown in Figure 2.4 and Figure 2.5 for the top and the bottom skin, respectively, are obtained.

\subsection{Thickness optimization procedure}

Once the optimized SST is obtained, the optimization of the thickness distribution can be started. In this section, a level-set method is introduced for the optimization 
Table 2.2: The result of the optimization problem defined in Equation (2.1) for the bottom skin.

\begin{tabular}{ccccc}
\hline Panel-set & $n_{0}$ & $n_{45}$ & $n_{90}$ & $\begin{array}{c}\text { Idealized number of plies (rounded) } \\
2\left(n_{0}+n_{45}+n_{90}\right)\end{array}$ \\
\hline 1 & 15.9 & 6.7 & 2.8 & 51 \\
2 & 1.7 & 8.3 & 1.6 & 23 \\
3 & 3.3 & 4.2 & 1.3 & 18 \\
4 & 7.7 & 2.4 & 1.3 & 23 \\
5 & 1 & 2 & 1 & 8 \\
6 & 1 & 2 & 1 & 8 \\
7 & 1 & 2 & 1 & 8 \\
8 & 1 & 2 & 1 & \\
\hline
\end{tabular}

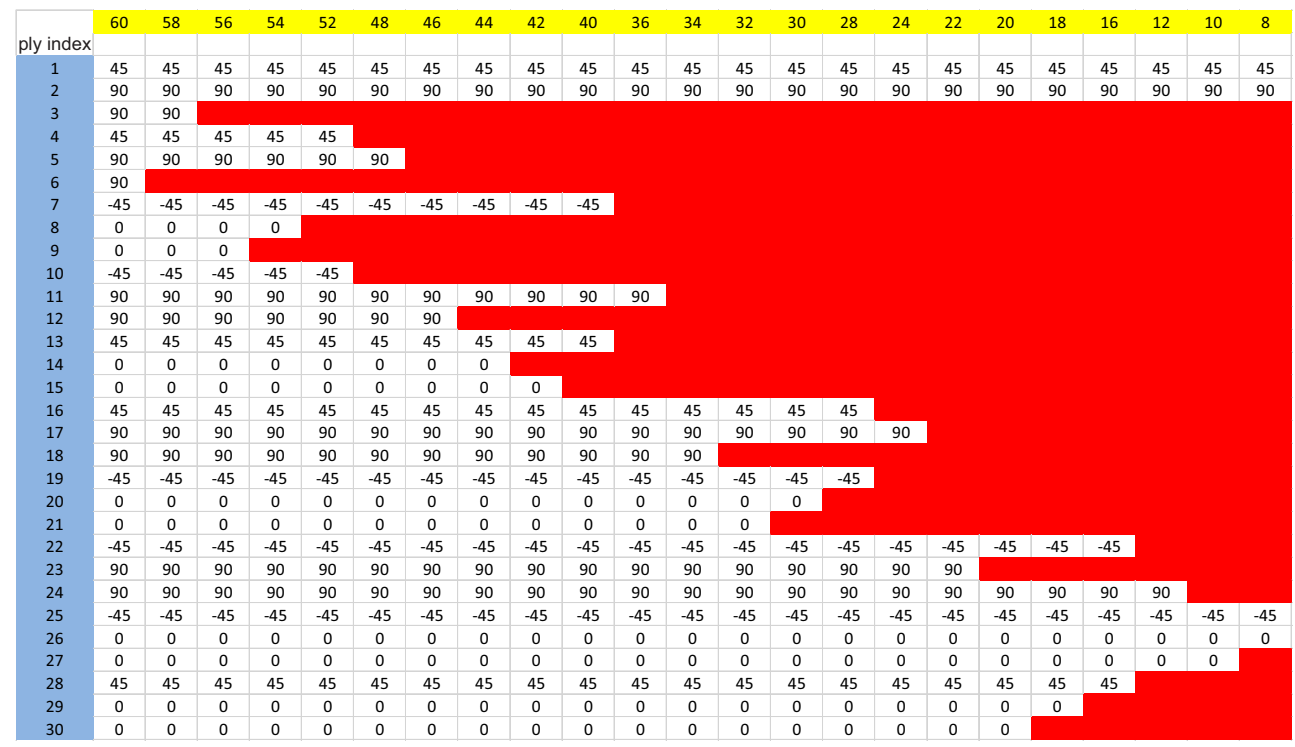

Figure 2.4: The optimized SST for the top skin. The first row shows the number of plies in each laminate. Due to symmetry, only the stacking sequences of half-laminates are shown. 


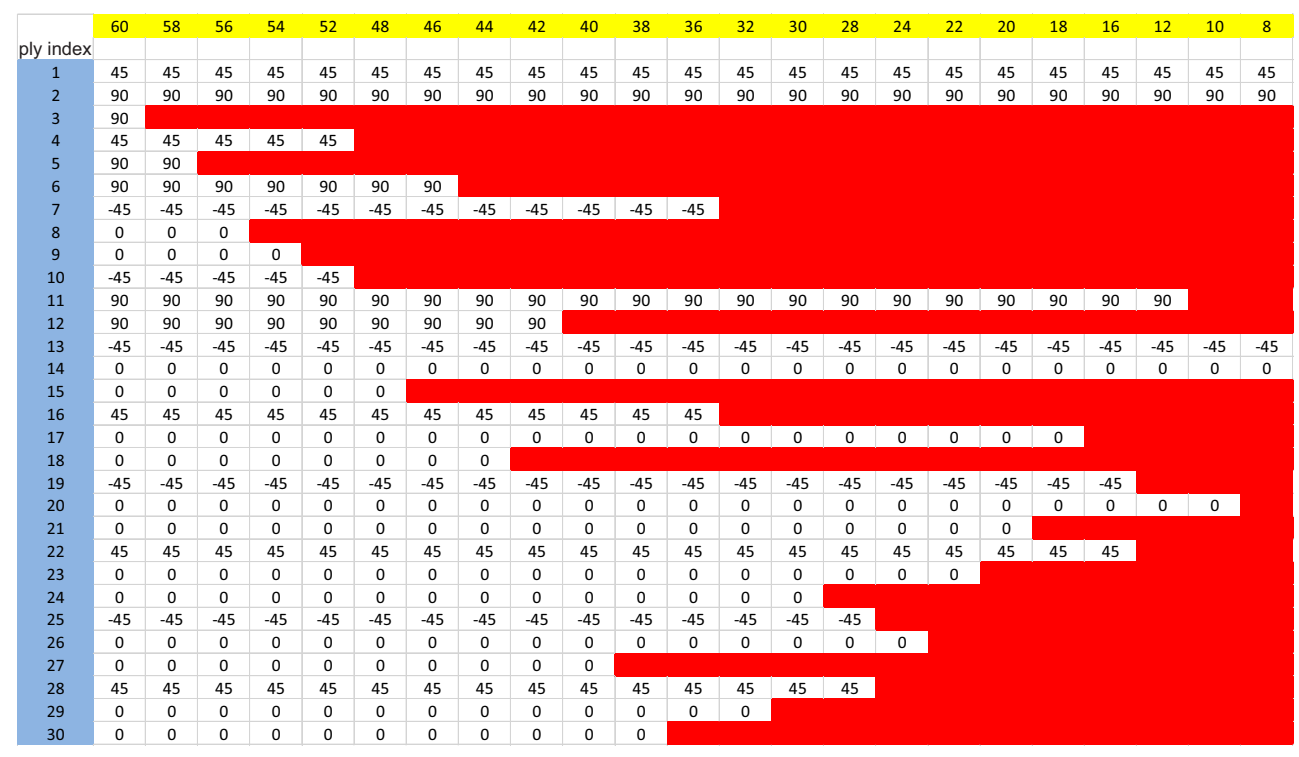

Figure 2.5: The optimized SST for the bottom skin. The first row shows the number of plies in each laminate. Due to symmetry, only the stacking sequences of halflaminates are shown.

of the thickness distribution using an SST.

\subsubsection{The proposed level-set-based method}

A single level-set function is used to determine the boundaries of laminates with different thicknesses in the composite structure. The level-set function $(\Phi)$ is discretized by interpolation among a limited set of $N_{\phi}$ design nodes with locations $X_{\phi}$. The design nodes are distributed over the structure. The values $\Phi\left(X_{\phi}\right)$ are the design variables in the optimization problem. The value of $\Phi$ at any location $X$ is obtained by the interpolation

$$
\Phi(X)=\sum_{n=1}^{N_{\Phi}} \Gamma^{n}(X) \Phi^{n}
$$

where $\Gamma^{n}(X)$ are interpolation functions and $\Phi^{n}$ represents $\Phi\left(X_{\phi}^{n}\right)$. In this research, we use bilinear interpolation functions on quadrilateral domains, spanning multiple finite elements. This means that the discretization of $\Phi$ is independent of the finite element discretization. The same $\Phi$ can be used on models of the same structure with different mesh size, e.g. a coarse model for compliance and a fine model for stress evaluation.

In the proposed method, the level-set function is used to select a ply stack from the (optimized) SST. The number of plies $N_{\text {plies }}$ of the laminate at any point $X$ in the 
design domain $\Omega$ is determined through the level-set function by:

$$
N_{\text {plies }}(X)=\left\{\max L V_{i} \mid L V_{i} \leq \Phi(X),\left(L V_{i} \in \mathrm{LV}\right)\right\}
$$

where LV represents the set of all ply stack values in the SST. According to Equation (2.4), the highest ply stack value below the level-set function at point $X$ gives the number of plies of the laminate covering point $X$. Figure 2.6(a) shows a level-set function covering a structure. The resulting thickness distribution is shown in Figure 2.6(b). The ply stacks are selected from the SST shown in Figure 2.4 or Figure 2.5. The LV set for the SST shown in Figure 2.4 is $\{8,10,12,16, \ldots, 60\}$ plies.

A larger number of design nodes can provide a more detailed design. However, this results in an increased computational time. The number and the location of the design nodes can be specified depending on the required details for the design.

Each finite element node will be assigned a level-set function value based on interpolation from the design node values. Within an element the interpolation of the value of the element nodes specifies the level-set function. Figure 2.7 shows this procedure schematically in three steps. Each finite element obtains area weighted stiffness data based on the area that each SST laminate covers in the element.

Using the proposed parametrization, the continuous change of a design variable influences the thickness distribution and the topology in the design. Therefore, a change in a design variable affects the elements stiffness matrices. This is the basis of the sensitivity analysis to iteratively update the design using any gradient based algorithm.

The validity of the proposed method is examined by solving the well-known Horseshoe Problem. This problem was first proposed by Soremekun et al. [5] and subsequently studied in $[3,4,6-9,23]$. Detailed information about the validation results can be found in Paper A.

Based on the proposed level-set-based method, different types of engineering problems can be solved. For detailed information on defining mass and compliance minimization problems, see Paper A, and Paper B, respectively.

The proposed level-set based method for the optimization of thickness distribution can also be employed for the shape and topology optimization of composite structures. Validation and examples in this respect are provided in Paper B. 


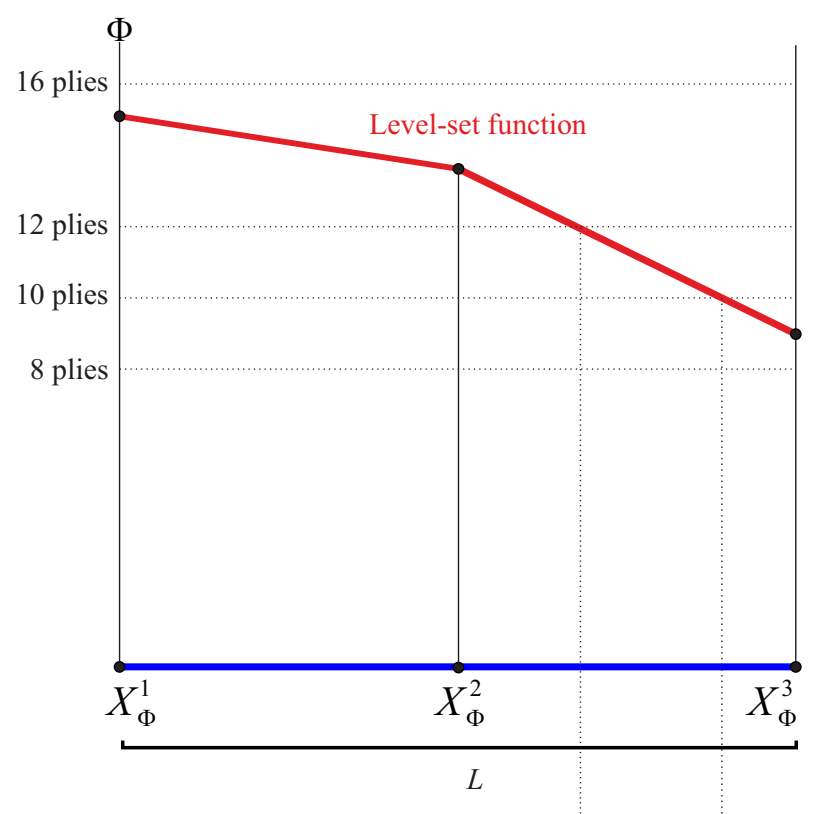

(a) Level-set function covering a 1D structure.

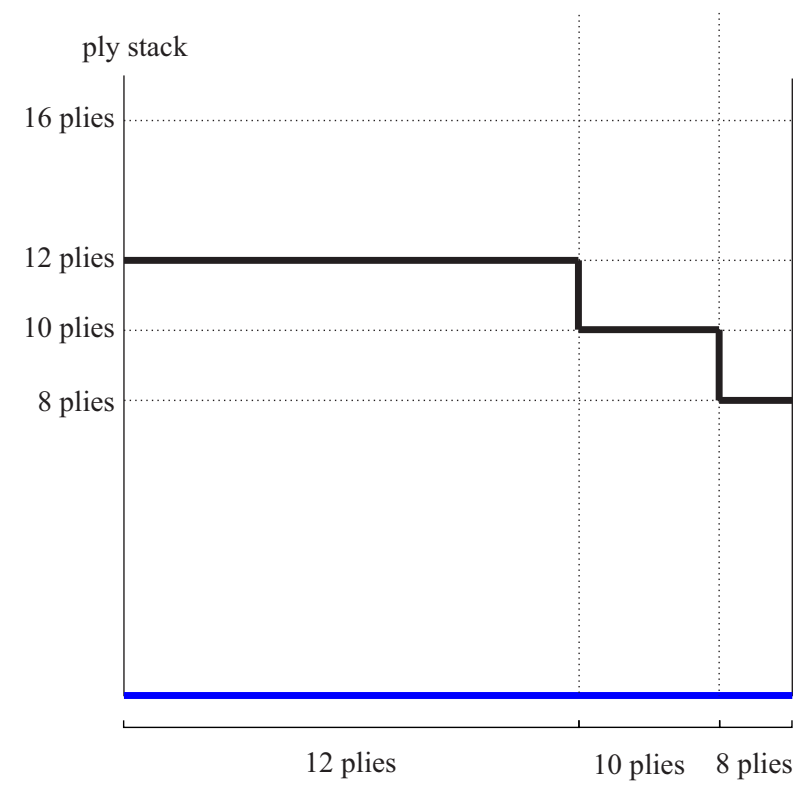

(b) Thickness distribution specified by the level-set function.

Figure 2.6: Determination of thickness distribution using a level-set function. The levels are the ply stack values in the SST shown in Figure 2.4. 


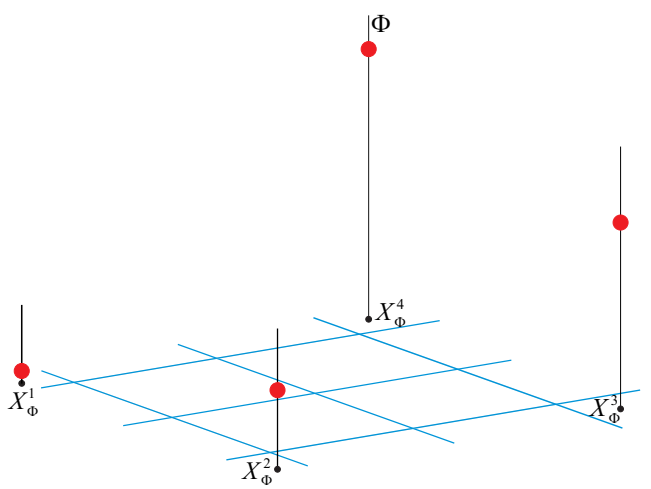

(a) Step 1: only the design nodes (at $X_{\phi}^{i}$ ) have levelset function values. These values are indicated by red dots.

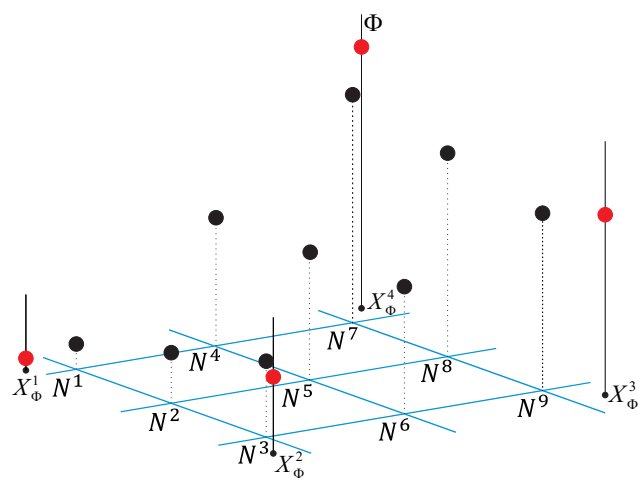

(b) Step 2: each finite element node $N^{i}$ obtains a level-set function value through the design node values interpolation.

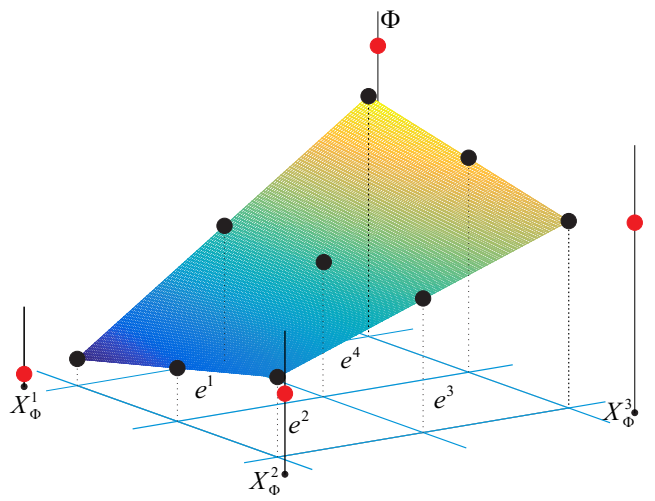

(c) Step 3: the level-set function within the element $e^{i}$ is defined by interpolating the element nodal values.

Figure 2.7: The interpolation procedure to define a level-set function using the design node values is shown in three steps. 


\section{References}

[1] J. Liu and Y. Ma. A new multi-material level set topology optimization method with the length scale control capability. Computer Methods in Applied Mechanics and Engineering, 329:444-463, 2018.

[2] L. A. Vese and T. F. Chan. A multiphase level set framework for image segmentation using the Mumford and Shah model. International Journal of Computer Vision, 50(3):271-293, 2002.

[3] J. Yang, B. Song, X. Zhong, and P. Jin. Optimal design of blended composite laminate structures using ply drop sequence. Composite Structures, 135:30 - 37, 2016.

[4] D. B. Adams, L. T. Watson, Z. Gürdal, and C. M. Anderson-Cook. Genetic algorithm optimization and blending of composite laminates by locally reducing laminate thickness. Advances in Engineering Software, 35(1):35-43, 2004.

[5] G. Soremekun, Z. Gürdal, C. Kassapoglou, and D. Toni. Stacking sequence blending of multiple composite laminates using genetic algorithms. Composite Structures, 56(1):53-62, 2002.

[6] F. X. Irisarri, A. Lasseigne, F. H. Leroy, and R. Le Riche. Optimal design of laminated composite structures with ply drops using stacking sequence tables. Composite Structures, 107:559-569, 2014.

[7] S. T. IJsselmuiden, M. M. Abdalla, O. Seresta, and Z. Gürdal. Multi-step blended stacking sequence design of panel assemblies with buckling constraints. Composites Part B: Engineering, 40(4):329 - 336, 2009.

[8] O. Seresta, M. M. Abdalla, and Z. Gürdal. A genetic algorithm based blending scheme for design of multiple composite laminates. In Collection of Technical Papers - AIAA/ASME/ASCE/AHS/ASC Structures, Structural Dynamics and Materials Conference, 2009.

[9] P. Jin, Y. Wang, X. Zhong, J. Yang, and Z. Sun. Blending design of composite laminated structure with panel permutation sequence. Aeronautical Journal, 122(1248):333-347, 2018. cited By 0.

[10] A. Carpentier, L. Michel, S. Grihon, and J. J. Barrau. Buckling optimization of composite panels via lay-up tables. In 3rd European Conference on Computational Mechanics Solids, Structures and Coupled Problems in Engineering, Lisbon, Portugal, June 2006.

[11] D. Liu, V. V. Toroporov, O. M. Querin, and D. C. Barton. Bilevel optimization of blended composite wing panels. Journal of Aircraft, 48(1):107-118, 2011.

[12] C. Kassapoglou. Design and Analysis of Composite Structures: With Applications to Aerospace Structures. John Wiley and Sons, second edition, 2013. 
[13] Y. M. Meddaikar, F. X. Irisarri, and M. M. Abdalla. Laminate optimization of blended composite structures using a modified Shepard's method and stacking sequence tables. Structural and Multidisciplinary Optimization, 55(2):535-546, 2017.

[14] F. X. Irisarri, D. M. J. Peeters, and M. M. Abdalla. Optimisation of ply drop order in variable stiffness laminates. Composite Structures, 152:791-799, 2016.

[15] M. Montemurro, A. Vincenti, and P. Vannucci. A two-level procedure for the global optimum design of composite modular structures-application to the design of an aircraft wing: Part 1: Theoretical formulation. Journal of Optimization Theory and Applications, 155(1):1-23, 2012.

[16] M. Montemurro, A. Vincenti, and P. Vannucci. A two-level procedure for the global optimum design of composite modular structures-application to the design of an aircraft wing: Part 2: Numerical aspects and examples. Journal of Optimization Theory and Applications, 155(1):24-53, 2012.

[17] S. T. IJsselmuiden, M. M. Abdalla, and Z. Gürdal. Optimization of variablestiffness panels for maximum buckling load using lamination parameters. AIAA Journal, 48(1):134-143, 2010.

[18] M. Montemurro and A. Catapano. A new paradigm for the optimum design of variable angle tow laminates. Springer Optimization and Its Applications, 116:375-400, 2016.

[19] M. Montemurro and A. Catapano. On the effective integration of manufacturability constraints within the multi-scale methodology for designing variable angle-tow laminates. Composite Structures, 161:145-159, 2017.

[20] S. W. Tsai and H. T. Hahn. Introduction of composite materials. Technomic Publishing Company, Lancaster, PA, 1980.

[21] V. B. Hammer, M. P. Bendsøe, R. Lipton, and P. Pedersen. Parametrization in laminate design for optimal compliance. International Journal of Solids and Structures, 34(4):415-433, 1997.

[22] F. Farzan Nasab, H. J. M. Geijselaers, I. Baran, and A. de Boer. Generating the best stacking sequence table for the design of blended composite structures. In Advances in Structural and Multidisciplinary Optimization: Proceedings of the 12th World Congress of Structural and Multidisciplinary Optimization (WCSMO12), pages 779-788, 2018.

[23] P. Jin, X. Zhong, J. Yang, and Z. Sun. Blending design of composite panels with lamination parameters. The Aeronautical Journal, 120(1233):1710-1725, 2016. 


\section{Optimization of interacting skins and ribs of a wing box}

The structural optimization of an aircraft wing is a highly complex problem. This is due to the large number of variables as well as structural and aerodynamics constraints influencing the design of skins and stiffeners. To make it computationally more efficient, a large problem can be decomposed into several smaller subproblems (subsystems) while preserving the couplings among these subproblems [1].

Quasi-separable decomposition [2], is a method that breaks a large-scale optimization problem into a system level (or global level) and several subsystems (or local level) optimizations where the subsystems are independent of each other. In this method, the design of a subsystem is performed by the local design variables and the global system variables, but no variable from the other subsystems [3]. A quasi-separable decomposition approach allows for solving the subsystems in parallel and thus enhances the efficiency of the optimization algorithm.

In present research, a quasi-separable decomposition approach is developed and applied to the optimization problem of the skins and ribs of a wing box (see Paper D for details). The proposed method decomposes each iteration of the wing box optimization into a system-level and subsystem optimization problem. At the system level, the wing box skins are optimized considering the effect of the design of the skins on the design of the ribs. The ribs are the subsystems of the optimization problem. Each rib has a local set of design variables and constraints which are influenced by the skin design variables, but not by the design variables from other ribs. The proposed strategy for the optimization of variable stiffness composite structures (see Chapter 2 ) is employed in both wing-level and rib-level optimization problems.

The rib-level and the wing-level optimization are coupled with each other via the crushing load (or Brazier load) [4,5] applied to the ribs (see Figure 3.1). The crushing load applied to the ribs is influenced by the wing box bending. The skin design affects the bending stiffness of the wing box and thus affects the crushing load applied to 


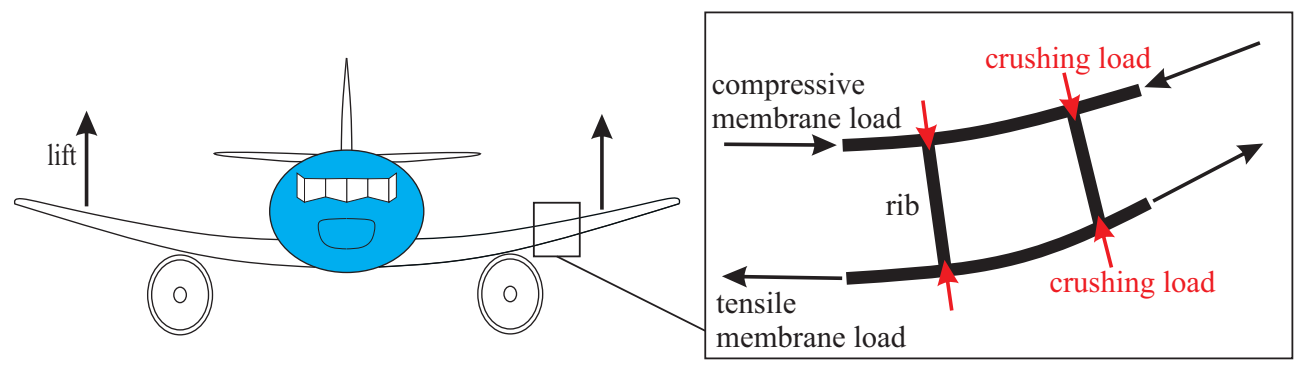

Figure 3.1: The components of the in-plane compressive and tensile loads cause crushing load on the ribs $[4,5]$.

the ribs. A set of coupling sensitivity calculations is required to represent the skinrib coupling. To enhance the numerical efficiency of this optimization problem, first the Lagrange multipliers of the optimized rib solution are used to obtain the design sensitivity of the ribs with respect to the loads. Second, a scheme based on Principal Component Analysis (PCA) is applied to compute the coupling sensitivities. The applicability of the proposed decomposition is examined by optimizing the skins and ribs of a wing box. In the following, the rib and the wing-level optimization and their coupling terms are discussed.

\subsection{Rib-level optimization}

The rib-level optimization is formulated as

$$
\begin{aligned}
\min & f_{\text {rib-level }}=W_{\text {rib }}\left(\Phi_{\text {rib }}\right) \\
\text { subject to } & g_{\text {rib }}^{i}\left(\Phi_{\text {rib }} ; \mathbf{L}_{\text {rib }}\right) \leq 0 \quad i=1 \text { to } m_{\text {rib }} \\
& \Phi_{\min } \leq \Phi^{n} \leq \Phi_{\max }
\end{aligned}
$$

where $W_{\text {rib }}$ represents the weight of the rib as a function of the rib design variables vector $\Phi_{\text {rib }} \cdot g_{\text {rib }}^{i}$ represents the $i^{\text {th }}$ constraint applied to the optimization problem of a rib, $\mathbf{L}_{\text {rib }}$ is the rib load vector, and $m_{\text {rib }}$ is the number of rib constraints. $\Phi_{\min }$ and $\Phi_{\max }$ denote respectively the lower bound and upper bound for the values at the design nodes. The load applied to each rib is kept constant in the rib-level optimization.

\section{$3.2 \quad$ Skin-rib coupling}

The following sensitivity of the optimized weight of a rib $\left(W_{\mathrm{rib}}^{*}\right)$ with respect to the skins' design variables needs to be calculated at the end of a rib-level optimization:

$$
\frac{\partial W_{\mathrm{rib}}^{*}}{\partial \Phi_{\mathrm{skin}}^{i}}=\frac{\partial W_{\mathrm{rib}}^{*}}{\partial \mathbf{L}_{\mathrm{rib}}} \frac{\partial \mathbf{L}_{\mathrm{rib}}}{\partial \Phi_{\mathrm{skin}}^{i}}
$$


According to Equation 3.2, for every perturbed skins' design variable, the rib has to be optimized with the updated rib load vector $\left(\mathbf{L}_{\mathrm{rib}}\right)$ so that the coupling sensitivities can be calculated. This requires a large number of computations. Here, the vector of Lagrange multipliers of the rib active constraints at the optimized design is used to calculate $\partial W_{\text {rib }}^{*} / \partial \mathbf{L}_{\text {rib }}$.

A number of constraint related finite element analyses (such as buckling or compliance), equal to the number of the skins' design variables is required for calculating the skin-rib coupling sensitivities. The analyses related to the coupling terms have to be performed in each full iteration (including rib-level and wing-level) of the optimization problem.

The sensitivity values of the rib crushing load with respect to the skins' design variables $\left(\partial \mathbf{L}_{\text {rib }} / \partial \Phi_{\text {skin }}^{i}\right)$ is required in the proposed decomposition method. As a result of performing such sensitivity analysis, a number of load sensitivity vectors equal to the number of design variables of the skins are obtained. The load sensitivity vectors might be correlated. If this correlation exists, it can be used to reduce the number of analyses required for calculating the coupling sensitivities. Here, a reduction technique based on the PCA is introduced to identify such correlation and use it to reduce the number of analyses required for calculating coupling sensitivities. Detailed information on calculating the coupling sensitivities can be found in Paper D.

\subsection{Wing-level optimization}

The wing-level optimization is formulated as:

$$
\begin{array}{lll}
\min & f_{\text {wing-level }}=W_{\text {Tskin }}\left(\Phi_{\text {Tskin }}\right)+ & W_{\text {Bskin }}\left(\Phi_{\text {Bskin }}\right)+\sum_{i=1}^{n_{\text {rib }}} W_{\text {rib }^{i}}^{*}\left(\mathbf{L}_{\text {rib }}\left(\Phi_{\text {skin }}\right)\right) \\
\text { s.t. } & g_{\text {Tskin }}^{i}\left(\Phi_{\text {Tskin }}, \mathbf{L}_{\text {Tskin }}\right) \leq 0 \quad i=1 \text { to } m_{\text {Tskin }} \\
& g_{\text {Bskin }}^{i}\left(\Phi_{\text {Bskin }}, \mathbf{L}_{\text {Bskin }}\right) \leq 0 \quad i=1 \text { to } m_{\text {Bskin }} \\
& \Phi_{\text {min }} \leq \Phi^{n} \leq \Phi_{\max }
\end{array}
$$

where $W_{\text {Tskin }}$ and $W_{\text {Bskin }}$ represent the weight of the top and the bottom skin, respectively, and $\Phi_{\text {skin }}=\left[\begin{array}{l}\Phi_{\text {Tskin }} \\ \Phi_{\text {Bskin }}\end{array}\right] . W_{\text {rib }^{i}}^{*}$ represents the optimized weight of the $i^{\text {th }}$ rib. The top skin and the bottom skin constraints are represented by $g_{\text {Tskin }}$ and $g_{\text {Bskin }}$, respectively. $\mathbf{L}_{\text {Tskin }}$ and $\mathbf{L}_{\text {Bskin }}$ represent the load vector applied to the top and the bottom skin, respectively. The number of the top and the bottom skin constraints is denoted by $m_{\text {Tskin }}$, and $m_{\text {Bskin }}$, respectively.

In the wing-level optimization, the effect of the skins' design variables on the optimized weight of the ribs needs to be calculated. Therefore, in Equation (3.3) the term $W_{\mathrm{rib}^{i}}$ is only a function of the skins' design variables.

The top and the bottom skin are also coupled through the loads. The effect of this coupling is implemented in the constraints of the optimization problem at the winglevel. Therefore, in Equation (3.3) the weight of either of the skins is only the function of that skin's design variables while the constraints of either of the skins are functions 
of both skins' design variables. Detailed information on calculation of the sensitivities of the objective and constraints functions can be found in Paper D. 


\section{References}

[1] B. Liu, R. T. Haftka, and L. T. Watson. Global-local structural optimization using response surfaces of local optimization margins. Structural and Multidisciplinary Optimization, 27(5):352-359, 2004.

[2] R. T. Haftka and L. T. Watson. Decomposition theory for multidisciplinary design optimization problems with mixed integer quasiseparable subsystems. Optimization and Engineering, 7(2):135-149, 2006.

[3] Q. Liu, M. Jrad, S. B. Mulani, and R. K. Kapania. Global/local optimization of aircraft wing using parallel processing. AIAA Journal, 54(11):3338-3348, 2016.

[4] L. S. Cecchini and P. M. Weaver. Brazier effect in multibay airfoil sections. AIAA Journal, 43(10):2252-2258, 2005.

[5] B. K. Stanford and P. D. Dunning. Optimal topology of aircraft rib and spar structures under aeroelastic loads. Journal of Aircraft, 52(4):1298-1311, 2015. 



\section{Discussion}

In this chapter, first the efficiency of the proposed optimization method in terms of computation cost is discussed, then the accuracy of the proposed method is addressed. Some additional remarks conclude this chapter.

\subsection{Efficiency of the optimization method}

The following characteristics make the proposed optimization method efficient in terms of computation cost.

\subsubsection{A fully gradient-based method}

Perhaps the most prominent reason for the efficiency of the proposed optimization method in terms of computation cost is that it is fully gradient-based. As mentioned earlier, due to the discrete nature of the variables involved in the design optimization of composite structures, GAs are often employed (see e.g. [1-7]). GAs require a large number of function evaluations which makes them inconvenient for practical applications. The proposed method for the optimization of a variable stiffness composite structure is, however, well suited for a gradient-based optimization algorithm. There are two reasons for this: 1- The optimization of the stacking sequences (which is a discrete problem due to the discrete values of the fiber orientations) is separated from the optimization of the thickness distribution. A gradient-based algorithm to obtain the idealized stiffness distribution is used for the optimization of the stacking sequences. The idealized stiffness distribution is then used by a deterministic algorithm to account for the design guidelines. 2- The proposed level-set-based method turns the discrete nature of the thickness distribution optimization problem into a continuous one through the way it parametrizes the problem. The thickness distribution optimization problem can be also solved using a gradient based optimization algorithm. 
To show the efficiency of the proposed method with respect to a GA, the number of required buckling analyses to solve the Horseshoe Problem (studied in Paper A) using the proposed method is compared with the number using a GA-based method from literature (Yang et al. [5]).

The total number of buckling analyses $\left(\Lambda_{\text {tot }}\right)$ in the proposed method is calculated as follows:

$$
\Lambda_{\text {tot }}=i t r \times\left(N_{\mathrm{D}}+1\right) \times \text { panel }+i t r \times \text { trial } \times \text { panel }
$$

where $i t r$ represents the number of iterations, $N_{\mathrm{D}}$ is the number of design nodes, panel is the number of panels, and trial denotes the number of trials in the line search procedure to find the proper step size. The number of step size trials in all iterations is no more than three. Thus trial is set at 3 and panel equals 18 in Equation (4.1). Equation (4.1) is first calculated for $i t r=6$ and $N_{\mathrm{D}}=12$ and then with $i t r=3$ and $N_{\mathrm{D}}=30$ (as discussed in Paper A). The summation of the two calculations gives the total number of 3564 buckling calculations for the whole optimization process using the proposed method.

In [5], the number of generations before convergence is reported to be 2500 for only balanced and symmetric laminates. Each generation includes 200 individuals. To evaluate each individual design, a number of buckling analyses equal to the number of panels must be performed. This means that the algorithm used in [5] requires 9 million buckling analyses which is almost 3000 times more than used in the proposed method. The buckling factor in [5] (and also in $[1-4,6]$ ) is obtained through an analytical equation for a simply supported panel under bi-axial compression. Every buckling calculation using the analytical method takes 0.001 second in an ordinary PC. Therefore, the 9 million calculations can be performed in a tolerable time (less than three hours). However, the Horseshoe Problem is a very simplified example. In reality, the load redistribution and the existence of the shear load require finite element calculation for buckling analysis. The finite element buckling analysis for each panel takes about four seconds on the same computer. Thus solving the Horseshoe optimization problem using the GA method in [5] and finite element calculations would take 10,000 hours for only symmetric and balanced laminates.

It is worth mentioning that increasing the number of laminate design guidelines, which was mentioned in Chapter 1 , has no effect on the number of calculations in the proposed method. This stands in contrast to the methods available in literature where the design guidelines are treated as constraints for the optimization problem. In [5], for example, 500 more generations are required when balance is added to the symmetry guideline. This can be translated into 1.8 million (500 generation $\times 200$ individuals $\times$ 18 panels) more buckling analyses by just adding one more constraint. An extremely high number of buckling analyses is expected if all guidelines are imposed for a real design.

\subsubsection{Straightforward in handling the blending guideline}

The proposed method for the optimization of the variable stiffness composite structure guarantees the manufacturability of the final design (blending) without extra 
computation cost for the optimization problem (see Paper A). This stands in contrast to the methods where blending is addressed by adding the continuity constraint to the optimization problem (see e.g. [8]).

\subsubsection{Fast in evaluating the performance of SSTs}

In the procedure of generating an optimized SST, as a result of solving the idealized thickness and stiffness distribution, some panels (or sets of panels) may obtain the same thickness value while having different values of $n_{0}^{j}, n_{45}^{j}$, and $n_{90}^{j}$. This means that, for a laminate with a certain thickness, different stiffness values are required in different panels (regions) of the structure. This suggests the existence of multiple SSTs (see Paper A for an example in this regard). A method is introduced to evaluate the performance of different SSTs quickly by defining a quality indicator (see Paper A). The advantage of using the proposed quality indicator is that different SSTs can be evaluated without performing a finite element analysis. This results in a cheap SST evaluation.

\subsubsection{Parametrized independently from the ply numbers and possible fiber orientations}

In the proposed method no restriction is imposed to the number of plies. Design variables can change continuously in the range of thinnest and the thickest laminates of the SST. This stands in contrast to the conventional density-based techniques for topology optimization of composite structures where a fixed design domain is required to be defined before starting the optimization. This means that a maximum number of layers has to be assumed and imposed on the problem. Therefore, if as a result of optimization, laminates thicker than the imposed maximum value are required, they cannot be prescribed by the optimizer. If the design domain were discretized into a larger number of layers, the number of design variables would have increased (as design variables are assigned to each element), resulting in a more expensive and complex optimization problem.

The conventional level-set method has been applied to the optimization of multiple material phases $[9,10]$. For that purpose, multiple level-set functions are required to be defined and assigned to each material phase. This results in an increased number of design variables corresponding to the number of material phases and consequently increases the computation cost related to the sensitivity analysis. In the proposed level-set method, however, only a single level-set function is defined to prescribe the boundary of multiple layers (phases). This results in a significantly reduced computation cost than when multiple level-set functions are used. Moreover, in the conventional level-set method topological derivatives are required to nucleate new holes $[11,12]$. Using the proposed method, the holes in the design appear without using topological derivatives (see Paper B). This also makes the proposed level-set method cheaper than the conventional level-set method. 


\subsubsection{Flexible in adding extra design variables}

A more detailed design is desirable as it ends up in a lighter design (when mass is the objective). A more detailed design requires more design variables in the proposed level-set method. As the computation cost in the proposed method is directly related to the number of design variables, a design engineer may prefer a cheaper problem at the cost of losing details in the design. To capture a detailed design while limiting the computation cost, the proposed level-set method allows the optimization problem to start with a reasonably small number of design variables. After some iterations, when the design has evolved towards the optimum design, more design variables can be added to the problem. This will result in a cheaper problem than when the optimization initially starts with a large number of design variables, because in the former the design is already close to the optimum point. The quantitative comparison in this regard is provided in Paper A and Paper B.

\subsubsection{Independent from the (finite element) mesh size of the structure}

During the optimization procedure, it may be required that a design is subjected to different types of (finite element) analyses (e.g. stress or stiffness). Adequate mesh density is required to obtain an accurate result from the finite element analysis. For example, stress analysis requires a finer mesh size than a stiffness analysis. As a dense mesh introduces more degrees of freedom and consequently more computation cost, it is desirable to use models with different mesh densities with respect to the type of analysis. In the proposed method, the level-set function can prescribe the same design for both fine and coarse models. Therefore, by selecting an appropriate finite element model, a cheaper optimization process can be achieved. To obtain the bending stiffness of a wing box, a coarse model is used in Paper D. On the other hand, a fine model for the skins is used to perform buckling and strain analyses. As a large number of bending stiffness analyses is required, a considerably cheaper problem has to be solved thanks to the proposed level-set-based parametrization.

\subsubsection{Applicable to the optimization problem of the interact- ing skins and ribs of an aircraft wing by suggesting a special approach}

Part of this research specifically addresses the optimization of an aircraft wing made of composite materials. A large number of design variables relating to the optimization of composite structures adds to the complexity and computation cost of a high dimensional wing optimization problem. Therefore, it is crucial to have an efficient design method. It has been discussed (in Paper D) that dividing a complex optimization problem into several smaller problems is in general easier to manage than when it is solved in one go. A decomposition technique for the optimization of interacting skins and ribs of a wing box is proposed in this research. Using the proposed method, the skins are optimized at the system-level, and the ribs are optimized as sub-systems 
of the optimization problem. The design of the ribs is affected by the design of the skins (through the crushing loads) while the ribs can be optimized independently of each other. This allows the ribs to be optimized in parallel or by using different computation sources. This, consequently, makes it possible to optimize such a complex problem in less time.

It is of high importance to preserve the relation between the optimization of the system (here, skins) and sub-systems (here, ribs) throughout the entire procedure. In the proposed decomposition method, the sensitivity of the optimized weight of the ribs to skin design variables needs to be calculated. The use of Lagrange multipliers of ribs active constraints is suggested to obtain the sensitivity of the optimized rib weight to a change in the ribs active constraints. In Paper D, it is discussed how the application of Lagrange multipliers reduces the number of required analyses.

A PCA reduction is used to approximate the skin-rib coupling sensitivities. In Paper $\mathrm{D}$, it is shown that the application of the proposed PCA method results in $33 \%$ fewer finite element analyses when calculating the sensitivity of one of the ribs' active constraints to the skins' design variables. It is discussed that the reduction in the number of analyses may increase when more design variables are assigned to skins.

\subsection{Accuracy of the optimization method}

Different aspects that influence the accuracy of the final design obtained using the proposed design method are discussed in this section.

\subsubsection{Global/local optimum}

A typical question about an optimized design obtained using a gradient-based algorithm is whether the final solution is a global optimum. Answering this question is in general a very challenging task. Nevertheless, one potential solution is to use the exhaustive search method. According to this method, all the local minimum points for the continuous objective function $f(X)$ in a closed and bounded feasible set must be found. The one which gives the lowest value is defined as the global minimum [13]. The difficulty in using this method is that there is no guarantee that all the local minima have been found. Apart from that, the optimization problem has to be started with many different initial designs such that all local minima are found. This is very costly for an engineering problem in practice. The other solution is to prove that the optimization problem is convex. If proved, any local minimum is also a global minimum. In that case, the Karush-Kuhn-Tucker (KKT) necessary conditions are sufficient for identification of the minimum point [13]. A convex optimization problem requires a convex objective function defined on a convex feasible design space. To have a convex design space, inequality constraints must be convex and equality constraints must be linear. To check the convexity of a function of $n$ variables, the Hessian matrix of the function must be calculated. The necessary and sufficient condition for the convexity of a function is that the Hessian matrix is positive semi-definite 
or positive definite. The convexity of the proposed method for the optimization of variable stiffness composite structures is discussed below.

As mentioned earlier, the proposed method for the optimization of variable stiffness composite structures consists of two steps: optimization of stacking sequences and optimization of the thickness distribution. To optimize the stacking sequences the optimization problem defined in Equation (2.1) has to be solved. The objective function for this problem is rewritten here:

$$
\min f=\sum_{j=1}^{N_{\mathrm{p}}}\left(n_{0}^{j}+n_{45}^{j}+n_{90}^{j}\right) S_{j} t
$$

where the design variables are $n_{0}^{j}, n_{45}^{j}, n_{90}^{j}$. As seen in Equation (4.2), the objective function is a linear function of design variables and thus convex. Therefore, the convexity of the optimization problem relies on the constraints. For the top skin, for example, buckling is considered as the constraint of the problem. The buckling factor of a shell-like structure is a function of the bending stiffness of the structure and the load distribution. The load distribution does not allow for a straightforward expression to determine whether a buckling problem is convex. Therefore, it is assumed here that both the stacking sequence optimization and the optimization of the thickness distribution have several local optima. Consequently, it is possible that the optimized designs presented in Part II of this thesis are local optima.

\subsubsection{Choice of the initial design}

As discussed before, the optimized solution may be trapped in a local optimum. Thus the choice of initial design variables may affect the quality of the optimized design. One possible solution for this issue, is to solve the entire optimization problem several times, each time with different initial values for the design variables. The design which provides the lowest value for the objective function is selected. This solution may be applicable when the optimization problem is simple. Solving the entire problem several times is a cumbersome task for complex optimization problems and there is still no guarantee of finding the global optimum.

Another solution can be prescribed particularly for the proposed parametrization for variable stiffness composite structures. The first part of the proposed method aims at obtaining the idealized stiffness and thickness distribution (see Paper A). The idealized thickness distribution provides a proper suggestion for the initial values for the design variables of the level-set-based thickness optimization. As the initial design of the level-set-based thickness optimization is close to the idealized thickness distribution (the initial design is already close to the optimized design), it can be expected that the final thickness distribution is a global optimum for the thickness distribution optimization problem. 


\subsubsection{Number of panels (or panel-sets)}

To obtain the idealized stiffness and thickness distribution a set of design variables (one design variable for each fiber orientation) per panel or per panel-set has to be defined. Therefore, panels or panel-sets (which includes several adjacent panels) must be defined first. As a result of solving the idealized optimization problem, a constant stiffness and thickness per panel (or panel-set) is obtained as the idealized design. Thus the size of the defined panels (or panel-sets) influences the idealized stiffness and thickness distribution results.

An exact solution to define the size of the panels cannot be prescribed. However, the load distribution over the structure provides a clue about the boundary of panels. For example, if a region in the structure is subjected to a tensile load and there is compressive load in its vicinity, separated panels should be assigned to the two regions. As the idealized design is not the final solution for the optimization problem, defining the panel boundaries based on the load distribution provides sufficient information to optimize stacking sequences to be used subsequently for the thickness optimization problem.

\subsubsection{Generating the SST}

After solving the optimization problem related to the idealized design, the stacking sequences must be found to match the idealized thicknesses and stiffnesses while blending and other design guidelines are fulfilled. As mentioned in Paper A and Paper $\mathrm{B}$, here, it is proposed to start from the thinnest laminate and add plies successively until the thickest laminate is reached. Due to the blending constraint, the stacking sequence of a newly generated laminate cannot be freely chosen to match the required idealized stiffnesses as it must share a large portion with the previously generated laminate. Therefore, generating the thickest laminate and successively dropping plies results in a different SST from when the thinnest laminate is generated and plies are added successively.

A substitute for the proposed method of generating the stacking sequences is to define an optimization problem with the goal of finding stacking sequences with the closest stiffness properties to the idealized ones. Blending as well as other suggested laminate design rules must be defined as constraints of this optimization problem. Although this procedure may result in an SST with a better quality, it is much more costly than the method proposed in this research.

\subsubsection{Mesh density}

In Paper D, it is mentioned that models with different element sizes can be used with respect to the analysis. For example, a coarser model is used for the stiffness analysis and a finer model is used for the buckling analysis of a wing box skin. As the proposed level-set method allows the same design to be applied to the models with different element sizes, it can reduce the computation cost of the optimization problem without losing accuracy. 


\subsubsection{Tracking buckling modes}

The order of the buckling mode shapes may change as the design of the structure changes. During the numerical sensitivity analysis, it is crucial that the two buckling factors being subtracted from each other, belong to the same mode shape. Therefore, when a new set of buckling factors is obtained after perturbing a design variable, their corresponding mode shapes must be extracted too. Eigenvectors represent the buckling mode shapes corresponding to each buckling factor. The eigenvectors include the values of the out of plane translational and rotational degrees of freedom. A modal assurance analysis is used to properly track the mode shapes (see Paper A).

\subsection{Additional remarks}

It may be desired to have a constant laminate thickness per each panel (the area limited by the stiffeners and ribs) in a stiffened structure. This means that the ply drop locations have to be limited to the boundaries of panels. Answering the question regarding the necessity of this requirement is outside the scope of the present study, but the proposed level-set based strategy cannot satisfy this guideline in its current form.

If several adjacent design variables obtain values close to each other, their incremental change does not change the thickness distribution. The reason is that the design variables with similar values are all between the same levels. Therefore, the resulting level-set function has no intersection with the levels. This results in zero sensitivity of the objective and constraint functions with respect to these design variables. Therefore, depending on the problem, a relatively large number of design variables may hinder the optimization procedure. 


\section{References}

[1] D. B. Adams, L. T. Watson, Z. Gürdal, and C. M. Anderson-Cook. Genetic algorithm optimization and blending of composite laminates by locally reducing laminate thickness. Advances in Engineering Software, 35(1):35-43, 2004.

[2] F. X. Irisarri, A. Lasseigne, F. H. Leroy, and R. Le Riche. Optimal design of laminated composite structures with ply drops using stacking sequence tables. Composite Structures, 107:559-569, 2014.

[3] S. T. IJsselmuiden, M. M. Abdalla, O. Seresta, and Z. Gürdal. Multi-step blended stacking sequence design of panel assemblies with buckling constraints. Composites Part B: Engineering, 40(4):329 - 336, 2009.

[4] O. Seresta, M. M. Abdalla, and Z. Gürdal. A genetic algorithm based blending scheme for design of multiple composite laminates. In Collection of Technical Papers - AIAA/ASME/ASCE/AHS/ASC Structures, Structural Dynamics and Materials Conference, 2009.

[5] J. Yang, B. Song, X. Zhong, and P. Jin. Optimal design of blended composite laminate structures using ply drop sequence. Composite Structures, 135:30 - 37, 2016.

[6] P. Jin, X. Zhong, J. Yang, and Z. Sun. Blending design of composite panels with lamination parameters. The Aeronautical Journal, 120(1233):1710-1725, 2016.

[7] P. Jin, Y. Wang, X. Zhong, J. Yang, and Z. Sun. Blending design of composite laminated structure with panel permutation sequence. Aeronautical Journal, 122(1248):333-347, 2018. cited By 0.

[8] B. Liu and R. T. Haftka. Composite wing structural design optimization with continuity constraints. In 19th AIAA Applied Aerodynamics Conference, March 2001.

[9] J. Liu and Y. Ma. A new multi-material level set topology optimization method with the length scale control capability. Computer Methods in Applied Mechanics and Engineering, 329:444-463, 2018.

[10] L. A. Vese and T. F. Chan. A multiphase level set framework for image segmentation using the Mumford and Shah model. International Journal of Computer Vision, 50(3):271-293, 2002.

[11] N. P. Van Dijk, K. Maute, M. Langelaar, and F. Van Keulen. Level-set methods for structural topology optimization: A review. Structural and Multidisciplinary Optimization, 48(3):437-472, 2013.

[12] V. J. Challis. A discrete level-set topology optimization code written in matlab. Structural and Multidisciplinary Optimization, 41(3):453-464, 2010.

[13] J. S. Arora. Introduction to optimum design. Academic Press, Boston, third edition, 2012. 



\section{Summary, conclusions and recommendations}

\subsection{Summary}

Section 2.1 and Section 2.2 of Chapter 2 describe the method for the optimization of variable stiffness composite structures. The details of this method can be found in Paper A and Paper B. The applicability of the proposed method in the optimization of large-scale structures subject to multiple load cases is investigated in Paper C. Chapter 3 summarizes a decomposition strategy that is particularly suitable for the optimization of an aircraft wing structure. The details on the decomposition strategy can be found in Paper D.

\subsection{Conclusions}

Based on the description provided in Chapter 2 and Chapter 3, and the results presented in Part II of this thesis, the following conclusions are drawn.

- A gradient-based method is highly recommended for the optimization of variable stiffness composite structures. The inherent complexity involved in the design of composite structures does not allow a GA-based method to be applied to real problems.

- The separation of the stacking sequence optimization from the thickness optimization is an effective approach for optimizing the variable stiffness composite structures where blending (manufacturability guideline) as well as several composite design guidelines must be satisfied. This conclusion is drawn based on the results presented in Paper A and Paper C. 
- The use of a predefined Stacking Sequence Table (SST) guarantees the blending guideline (in addition to other design guidelines) without adding extra constraints to the optimization problem. The procedure of generating an SST can be easily adjusted to ignore some or add extra guidelines. A predefined (optimized) SST is a significant aid when extra (non-generic) constraints for the stacking sequences are required (see Appendix B where a horizontal stabilizer, which includes a left and a right side, requires laminates in each side with plies extended to the opposite side of the structure).

- The proposed level-set method gives continuous ply drop locations; i.e. the ply drop locations are not limited to panel boundaries in a stiffened structure. A design with ply drop locations limited to panel boundaries can be obtained by modification of the optimization result.

- The proposed level-set method is flexible in terms of the number and the location of design nodes. If in a region of the structure more details are required to be captured, more design nodes can be added locally. The computation time in the proposed method is directly related to number of design nodes in the level-set-based thickness optimization problem. The overall convergence time of a problem can be decreased as part of the optimization procedure can be performed with a smaller number of design nodes.

- The choice of the initial design of the level-set-based optimization may affect the final design of the problem. As for the initial design, it is suggested to assign values to design variables such that the resulting (area weighted average) thickness distribution is close to the idealized thickness distribution.

- The proposed level-set method can be used for solving engineering problems where structural mass (or volume) is either the objective or the constraint of the problem. In this thesis, mass minimization subject to stability and stiffness constraints, and compliance minimization subject to structural volume constraint were investigated.

- Flat and curved composite structures can be optimized using the proposed levelset method (as long as fibers are not steered). In this thesis, aircraft skins as a collection of flat panels and ribs were optimized, but an aircraft fuselage or a wind turbine blade can be optimized using this method as well.

- The proposed method can optimize the shape and the topology of a composite structure. A detailed topology optimization requires more design variables.

- When optimizing the interacting skins and ribs of an aircraft wing, the entire optimization time is reduced considerably when ribs are optimized separately and in parallel. The proposed decomposition scheme allows for the ribs to be optimized separately and in parallel.

- When optimizing the interacting skins and ribs of an aircraft wing, the components of the rib load vector derivatives with respect to the skins' design variables may be correlated. If this is the case, the proposed reduction scheme based on 
Principal Component Analysis (PCA) can be used to reduce the number of analyses required for evaluating the skin-rib coupling.

\subsection{Recommendations}

The smeared stiffness method is used to estimate the extensional (A) and the bending (D) stiffness matrices without knowing the stacking sequence of a laminate. This is achieved by assuming a homogeneous section for the layups. This assumption results in less accurate values for the bending stiffness matrix. For a future work, it is recommended to employ the lamination parameters [1]. Using lamination parameters as design variables, the idealized design can be obtained more accurately with a gradient-based optimization algorithm.

The sensitivity analysis to find a search direction is performed using the numerical finite difference method in the present research. Depending on the type of constraint, the response of the system before and after perturbing each design variable is obtained using the finite element analysis. This requires solving the finite element analysis system a number of times equal to the number of design variables +1 , in each iteration of the optimization problem. An efficient alternative is to derive the updated stiffness matrix (from the perturbed design variable) and perform sensitivity analysis using the system stiffness matrix before and after perturbation. The finite element system is not required to be solved in this alternative method. This would result in a cheaper sensitivity calculation [2].

For the buckling constraint in particular, in addition to the stiffness matrix, the stress stiffness matrix of the system changes. This is due to the change in the load distribution. Deriving an updated stiffness matrix is simple. However, deriving the stress stiffness matrix from a commercial finite element package is not straightforward. Finding a way to derive the stress stiffness matrix efficiently can be pursued in a future study.

A model reduction scheme based on PCA is developed in the present research to reduce the number of finite element analyses required for obtaining the skin-rib coupling sensitivities (see Paper D). It is suggested to develop a similar model reduction scheme as a future work and apply it to reduce the number of load cases if a correlation exists among the load cases. 


\section{References}

[1] D. Liu, V. V. Toroporov, O. M. Querin, and D. C. Barton. Bilevel optimization of blended composite wing panels. Journal of Aircraft, 48(1):107-118, 2011.

[2] W. Korbee. Semi-analytical buckling sensitivity calculation for the optimization of composite structures, MSc thesis, University of Twente, 2018. 


\section{Part II}

\section{Papers}





\section{Paper A}

\section{A level-set-based strategy for thickness optimization of blended composite structures}

This chapter is based on the paper 'A level-set-based strategy for thickness optimization of blended composite structures', published in: 'Composite Structures', Vol. 206 (2018) 903-920. Copyright by (C2018 Elsevier Ltd, https://doi.org/10.1016/j.compstruct.2018.08.059. Readers must contact the publisher for reprint or permission to use the material in any form. 



\title{
A level-set-based strategy for thickness optimization of blended composite structures
}

\author{
F. Farzan Nasab ${ }^{1)}$, H.J.M. Geijselaers ${ }^{1)}$, I. Baran ${ }^{1)}$, R. Akkerman ${ }^{1)}$, A. \\ de Boer ${ }^{1)}$
}

Composite Structures, Volume 206, December 2018

${ }^{1)}$ Applied Mechanics, Engineering Technology, University of Twente, Enschede, The Netherlands

\begin{abstract}
An approach is presented for the thickness optimization of stiffened composite skins, which guarantees the continuity (blending) of plies over all individual panels. To fulfill design guidelines with respect to symmetry, covering ply, disorientation, percentage rule, balance, and contiguity of the layup, first a stacking sequence table is generated. Next, a level-set gradient-based method is introduced for the global optimization of the location of ply drops. The method aims at turning the discrete optimization associated with the integer number of plies into a continuous problem. It gives the optimum thickness distribution over the structure in relation to a specific stacking sequence table. The developed method is verified by application to the wellknown 18-panel Horseshoe Problem. Subsequently, the proposed method is applied to the optimization of a composite stiffened skin of a wing torsion box. The problem objective is mass minimization and the constraint is local buckling.
\end{abstract}




\section{Introduction}

The application of composite structures in aerospace industry has noticeably increased in recent years. Designing such structures with respect to necessary manufacturing and design guidelines with minimum mass involves a large number of design variables and constraints. This results in a highly challenging optimization problem $[1-3]$. To meet a specified strength with minimum mass, a laminate design procedure may require determination of the total number of plies and the sequence of fiber orientation angles as design variables. Also, several strength related guidelines have to be satisfied. These guidelines are discussed in detail in [4, 5]. Moreover, manufacturability of the designed structure must be guaranteed. In a large scale structure, different regions may be subject to different loads. In an optimized design a laminate thickness may vary all-over the structure depending on the distributed loads. Also, for large scale composite structures, such as an aircraft wing or fuselage, stiffeners are added to enhance structural performance in carrying compressive and tensile loads. The stiffeners divide the structure into smaller panels. To ensure manufacturability of the composite skin it is crucial for the plies to be continuous among adjacent panels while the laminate thickness varies. Continuity of plies in adjacent panels, which is commonly referred to as blending [6], is a particularly difficult constraint to deal with [3]. Blending has to be satisfied in addition to the earlier mentioned strength related guidelines. Designing panels individually using local loads results in designs with significant manufacturing difficulties. The reason is that the resulting stacking sequences of laminates in adjacent panels may differ considerably [7]. Therefore, various methods have been proposed to address the global optimization of composite skins taking blending into account, see e.g. [8-11]. Liu and Haftka [12] introduced blending as a constraint to the optimization problem. They proposed a two-level algorithm. At the global level optimization, the continuous number of each ply in each panel is obtained. At the local level, those continuous numbers are rounded such that a genetic algorithm (GA) can prescribe blended stacking sequences for each panel. The rounding of the ply stack numbers, however, can cause internal panel load redistribution and subsequently causes constraints such as strain or buckling to be violated [12]. Adams et al. [8] introduced the 'edit distance' method in combination with a GA. In this study, a set of panel populations evolves to converge to a globally blended solution. In their research, the 'edit distance' is a blending indicator of the stacking sequences in adjacent panels. Designs with a higher degree of blending are rewarded via a fitness function. However, a large number of constraints was required to enforce blending. This made the optimization problem very difficult to be solved with any optimization algorithm [7]. In their later works [7, 13], Adams et al. introduced the concept of the guide-based blending in which the thickest stacking sequence is called the guide laminate and the stacking sequence of the laminates in all panels is obtained by dropping plies from the guide laminate. The method ensures the continuity of plies across neighboring panels without adding extra blending constraint. However, being restricted to a certain trend of dropping plies, which results in either outwardly or inwardly blended laminates, it ignores parts of the feasible region in the design space [1]. Zehnder and Ermanni [14, 15] introduced the patch concept for a globally blended design. In their work, instead of panel wise optimization of each laminate, 
the structure is treated as several globally extended layers that will be assembled together. The globally extended layers are called patches and are characterized by their geometry, material, and material orientation. Studying the structure globally has an advantage over panel wise optimization. A change of the stiffness of a panel also changes the local load distribution over the panels [16]. This invalidates the panel wise optimized design in terms of global constraint satisfaction and raises the need for a multi-level procedure. The patch concept is also attractive as the globally blended design can be obtained without narrowing the feasible region of the design space as happens when the ply drop is restricted to a specific pattern. Nevertheless, the patch concept suggests a large number of design variables which makes the optimization problem difficult to be solved [17].

Density-based topology optimization was originally introduced to solve for a twophase solid-void problem [18, 19]. The interpolation schemes such as SIMP (Solid Isotropic Material with Penalization) [19, 20] (see [21, 22] for recent advancements in the SIMP method) and RAMP (Rational Approximation of Material Properties) [23] are used to relax the discrete nature of the solid-void optimization problem. The interpolation schemes were subsequently extended to account for multiple phases in a design domain, see e.g. [24-27]. The application of topology optimization to laminated composites considering manufacturing constraints is investigated in Sørensen and Lund [28], Sørensen et al. [29], Sørensen and Stolpe [30], and Lund [31]. Recently, Allaire and Delgado [3] investigated the optimal design of a composite laminate by introducing the shape and the topology of each ply as design variables in addition to the fiber orientation angles and the sequence of the stack. Their proposed algorithm is, however, unable to prescribe a blended design[3].

A convenient aid in globally blended design optimization is the notion of the stacking sequence table (SST) introduced by Carpentier et al. [32]. The SST is a reference table for the stacking sequence of laminates with different thicknesses where a thicker laminate is obtained by adding plies to a thinner one resulting in admissible stacking sequences. The SST idea using a GA and the concept of the sequence of ply drops are investigated in $[2,17]$ and [1], respectively. Meddaikar et al. [2] combined a GA for SST optimization with a structural and a load approximation scheme. In their work, the approximation scheme is shown to be efficient in terms of computation cost.

The idea of the SST is shown to be practical to obtain optimized blended designs $[2,17]$. However, the ply drop locations in a fully GA-based final design are restricted to pre-specified locations. The reason is that the discrete nature of a GA does not allow for continuous ply drop locations. Irisarri et al. [33] have recently carried out a study to develop an optimization algorithm based on SST in which the location of the ply drop is not pre-specified but obtained using topology optimization.

The majority of the algorithms dedicated to global optimization of blended stiffened composite structures are fully or partially dependent on evolutionary algorithms $[1,7,12,17,34-37]$, typically the GAs, to deal with the discrete nature of the variables in designing a composite laminate (the use of evolutionary algorithms for the optimization of stacking sequences is reviewed in $[6,38]$ ). However, a GA is generally more time consuming than a gradient-based algorithm as there is a large number of designs to be analyzed [2]. Furthermore, to avoid narrowing the feasible region of the 
design space, ply drop locations do not have to be pre-specified. The ply drop location can be a continuous variable which suggest a gradient-based optimization algorithm. For the aforementioned reasons, the current research aims at investigating a problem parametrization that is suitable for the application of a gradient-based optimization algorithm. The proposed method is capable of generating a globally blended design at a limited computation cost while all other manufacturing guidelines are also satisfied.

The proposed approach separates the optimization of the stacking sequences from the optimization of the thickness distribution. A method has been developed by the authors to generate laminates with desired stacking sequences with respect to the optimization problem [39].

The present research is mainly focused on optimizing the thickness distribution with a given (fixed) set of stacking sequences. To this end, first, an SST is generated based on an estimation about the optimized stiffness and thickness distribution over the structure. The laminates in an SST satisfy symmetry, covering ply, disorientation, percentage rule, balance, and contiguity of the layup. Next, a novel level-set gradient-based method is introduced for the global optimization of the locations of the ply drops. A single function delineates the span of multiple levels where each level represents a ply in a stiffened composite skin. This stands in contrast to the studies where multiple level-set functions are used to represent multiple material phases $[40,41]$. The proposed method aims at turning the discrete optimization problem associated with the integer number of plies into a continuous problem. This is done through the way the problem is parametrized; the design variables are never rounded in this approach. The level-set function gives the optimum thickness distribution over the structure for a specific SST. The idea of combining an SST with an optimization algorithm to obtain the span of each ply is close to that introduced in [33]. However, the presented level-set method benefits from a straightforward procedure compared to rather complex topology optimization technique used in [33]. This allows convenient application of the method to the optimization of large scale structures. The developed method is verified by its successful application to the well-known horseshoe panel optimization problem studied in $[1,7,11,17,34-37]$. To investigate the performance of the method in dealing with a real problem, the proposed method is then applied to the layup optimization of a composite skin of a wing. Local buckling is considered as the constraint of the problem and a standard finite element package is used to calculate buckling factors.

\section{Generating a stacking sequence table (SST)}

An SST is a reference table for the stacking sequences of laminates with different thicknesses. Each column of the SST represents the stacking sequence of a certain number of plies. To guarantee the blending of a design, a thicker stacking sequence can only be obtained through adding plies to a thinner one [1]. To keep the design blended during the optimization process, the laminates across the structure are only allowed to be selected from the SST. Industrial requirements impose that the ply angles should be selected from a limited set $[17,42]$. In the present study, the ply orientations are 
limited to the set $\left\{0^{\circ}, \pm 45^{\circ}\right.$, and $\left.90^{\circ}\right\}$ of angles [42, 43]. Every laminate in an SST may be required to satisfy a number of strength related guidelines. In the present study, the following conventional guidelines proposed and applied in [2, 17, 33, 42, 43], are imposed in an SST:

- Symmetry, the laminate should be symmetric with respect to its center line.

- Covering plies, the outermost ply has to have the orientation of either $+45^{\circ}$ or $-45^{\circ}$.

- Disorientation, the maximum orientation difference of two adjacent plies is $45^{\circ}$.

- Percentage rule, the number of plies of a certain orientation has to be at least $10 \%$ of the total number of plies in a laminate.

- Balance, the total number of plies with $+45^{\circ}$ orientation in a laminate is equal to the total number of plies with $-45^{\circ}$ orientation.

- Contiguity, not more than 4 successive plies with a same orientation are allowed to stack together.

In general, imposing the aforementioned guidelines has a large influence on the complexity, computation cost, and the quality of the optimum design of the optimization problem (see e.g. $[44,45]$ ). The proposed method to generate an SST can be simply adapted to ignore or relax any of the aforementioned strength related guidelines.

A 2-step method was presented by the authors to generate an SST where the fiber orientations have to be selected from a limited set of angles [39]. As generating the stacking sequences is a key part of the optimization problem of composite structures, the (modified) 2-step approach is described in the following.

\subsection{Step 1: obtaining the optimized stiffness and thickness distribution (idealized design)}

To generate an SST, first the optimized stiffness and thickness distribution are estimated [33, 42, 46-48]. To this end, lamination parameters [49, 50], polar parameters $[47,48]$, or the smeared stiffness method [42] can be used. The smeared stiffness method requires fewest parameters and is computationally least expensive. Therefore, it has been selected to obtain an estimate of the optimized stiffness and thickness distribution (idealized design [33]).

The smeared stiffness method is used to estimate the extensional (A) and the bending (D) stiffness matrices without knowing the stacking sequence of a laminate. This is achieved by assuming a homogeneous section for the layups [42]. According to the classical laminate theory [51], the matrix $\mathbf{A}$ is defined as:

$$
\mathbf{A}=h\left(\sum_{k=1}^{N} \frac{\left(\bar{Q}_{i j}\right)_{k}}{N}\right), \quad i=j=1,2,6
$$


where $N$ and $h$ represent the total number of plies and the total thickness of the laminate, respectively. $\bar{Q}_{i j}$ represents the transformed plane stiffness [51].

Using the material homogeneity assumption [42], the bending stiffness matrix (D) can be obtained through:

$$
\mathbf{D} \approx \mathbf{A} h^{2} / 12
$$

Liu et al. [42] proposed solving the following optimization problem to obtain an estimation about the thickness and the stiffness distribution over the structure:

$$
\min f=\sum_{j=1}^{N_{\mathrm{p}}}\left(n_{0}^{j}+n_{45}^{j}+n_{90}^{j}\right) S_{j} t
$$

where $N_{\mathrm{p}}$ is the total number of panels, $S_{j}$ is the area of panel $j$, and $t$ is the ply thickness. The number of plies of each orientation in each panel (or a set of panels), $n_{0}^{j}, n_{45}^{j}, n_{90}^{j}, j=1, \ldots, N_{\mathrm{p}}$ are the design variables of this optimization problem. Due to the assumed balance guideline, the number of $+45^{\circ}$ plies has to be equal to the number of $-45^{\circ}$ plies. Therefore, $n_{45}^{j}$ is defined to be the sum of $+45^{\circ}$ and $-45^{\circ}$ plies.

Here, the constraints of the optimization problem in Equation (3) are defined as follows:

$$
\begin{array}{cl}
g_{i} \leq 0 & i=1 \text { to } N_{\mathrm{c}} \\
n_{0}^{j} / N^{j} \geq 0.1 & j=1 \text { to } N_{\mathrm{p}} \\
n_{45}^{j} / N^{j} \geq 0.2 & j=1 \text { to } N_{\mathrm{p}} \\
n_{90}^{j} / N^{j} \geq 0.1 & j=1 \text { to } N_{\mathrm{p}}
\end{array}
$$

where $g_{i}$ is a buckling constraint (defined in Section 3.3), $N_{\mathrm{c}}$ represents the number of required buckling constraints, and $N^{j}=n_{0}^{j}+n_{45}^{j}+n_{90}^{j}$. As it can be seen in Equation (4), it is required that the percentage of the plies of each orientation is $\geq 10 \%$ in each panel.

Solving the optimization problem defined in Equation (3) gives an estimation about the 'idealized' stiffness and thickness distribution. The output of this step is a table called SST-data. In an SST-data table, each thickness value appears once and a unique AD stiffness vector is assigned to every (rounded to the nearest integer) thickness value. Some panels (or sets of panels) may obtain the same thickness value while having different values of $n_{0}^{j}, n_{45}^{j}$, and $n_{90}^{j}$. This means that, for a laminate with a certain thickness, different stiffness values are required in different panels (regions) of the structure. This suggests the existence of multiple SST-data tables.

\subsection{Step 2: fitting the stacking sequences}

In the second step, the SST-data table(s) obtained in step 1, is used to generate the stacking sequence of laminates with different thickness values. In the procedure of generating the stacking sequences, all required laminate design guidelines have to be satisfied. 
All valid laminates (laminates that satisfy the strength related guidelines) with the thickness equal to that of the thinnest laminate obtained in the step 1 are generated (see Figure 1 for details on this procedure).

Among the set of the valid thinnest laminates, the one which has the closest stiffness values to those estimated for the same thickness laminate in step 1, is selected. A Root Mean Square Error (RMSE) of the components of the $\mathbf{A}$ and the $\mathbf{D}$ matrices was used to identify the laminate with the desired stiffness values.

To generate a thicker laminate, a ply (or two plies in case the fiber orientations are $-45^{\circ}$ and $+45^{\circ}$ ) has to be added to a thinner laminate. All valid laminates, according to the required guidelines, are generated. From the set of the newly built laminates the one with stiffness values closest to those of the laminate with the same thickness in the SST-data table is selected. This procedure continues until a laminate with the thickness equal to that of the thickest laminate in the SST-data table is reached.

It is possible that as a result of adding a ply to a thinner laminate, the thickness of the newly built laminates does not exist among the thickness values in the SST-data table. In this case, the stiffness values of the laminate with the closest thickness values in the SST-data table are (linearly) interpolated and used to select the fittest laminate.

Here, the SST was generated by successively adding plies to the thinnest laminate. Alternatively, the thickest laminate could have been generated from which plies had to be dropped successively. However, as the number of valid thinnest laminates is smaller than that of thickest laminates, it is cheaper to start with the thinnest laminate. The advantage of fitting an SST the way discussed is that it can be generated quite cheaply.

In case multiple SST-data tables are obtained from step 1, multiple SSTs (corresponding to each SST-data table) can be generated quickly using the proposed fitting method. To have an indication about the performance of the $k^{\text {th }}$ SST, using the idealized thickness distribution, the structure has to be covered with the stacking sequences of the $k^{\text {th }}$ SST. For this structure, the following expression has to be calculated:

$$
\eta_{i}^{k}=w_{i}^{T} \mathbf{K}_{\mathrm{B}}^{k} w_{i} \quad, \quad i=1 \text { to } N_{\mathrm{c}}
$$

where $w_{i}$ represents an eigenvector obtained as a result of solving the optimization problem defined in Equation (3). $\mathbf{K}_{\mathrm{B}}^{k}$ represents the bending stiffness of the structure when it is covered with the stacking sequences of the $k^{\text {th }}$ SST and the idealized thickness distribution is applied. The SST which maximizes the minimal $\eta_{i}^{k}$ is selected as the best generated SST.

Laminates with the same thickness but in different SSTs are required to satisfy the percentage rule guideline. Therefore, the number of plies of each orientation angle is almost equal for the laminates with the same thickness value. This means that, the in-plane stiffness for equally thick laminates in different SSTs is almost similar. Thus it can be assumed that the load redistribution is negligible as a result of using the same thickness distribution but stacking sequences from different SSTs.

The advantage of using the quality indicator defined in Equation (5) is that different SSTs can be evaluated without performing a finite element analysis. This results in a cheap SST evaluation. 
Figure 1 shows the flowchart of the 2-step procedure of generating an SST.

As mentioned before, some of the aforementioned strength related guidelines may not be required. As the major part of generating an SST concerns searching for laminates that satisfy the required guidelines (see Figure 1), the search criteria can be easily adapted to only select the required laminates.

An SST, in general, can include symmetric laminates with even and odd number of plies. However, only symmetric laminates with even number of plies are used.

An SST, as an example, is shown in Figure 2. Fields marked red indicate dropped plies and ply indices (first column from left) are in the ascending order from the outer most ply towards the laminates center.

\section{Level-set-based thickness optimization}

Using an SST, the optimization problem will be simplified to determine the optimum value of the thickness (from the SST) that has to be placed in each region of the structure. The boundaries of the stacks from the SST have to be determined and as long as laminates are selected from the SST, it is guaranteed that all guidelines are satisfied and also the final design is blended. Nevertheless, working with an SST demands dealing with discrete variables which cannot be done in the framework of gradient-based algorithms. In the following we introduce an efficient level-set method which turns the discrete nature of the above optimization problem into a continuous problem.

\subsection{The proposed level-set method}

An auxiliary level-set function is used to determine the boundary of each stacking sequence of an SST. The level-set function $(\Phi)$ is discretized by interpolation among a limited set of $N_{\phi}$ design nodes $X_{\phi}^{n}$ distributed over the structure. The values $\Phi^{n}=\Phi\left(X_{\phi}^{n}\right)$ are the variables in the optimization. The value of $\Phi$ at any location $X$ can then be found by the interpolation

$$
\Phi(X)=\sum_{n=1}^{N_{\Phi}} \Gamma^{n}(X) \Phi^{n}
$$

where $\Gamma^{n}(X)$ are interpolation functions. Here, bilinear interpolation functions on quadrilateral domains, spanning multiple finite elements are used. The discretization of $\Phi$ is independent of the finite element discretization. The same $\Phi$ can be used on models of the same structure with different mesh sizes, e.g. a coarse model for stiffness and a fine model for stress evaluation.

In the proposed method, the level-set function is used to select a ply stack from the SST. The number of plies $N_{\text {plies }}$ of the laminate at point $X$ is determined through the level-set function by:

$$
N_{\text {plies }}(X)=\left\{\max L V_{i} \mid L V_{i} \leq \Phi(X),\left(L V_{i} \in \mathrm{LV}\right)\right\}
$$




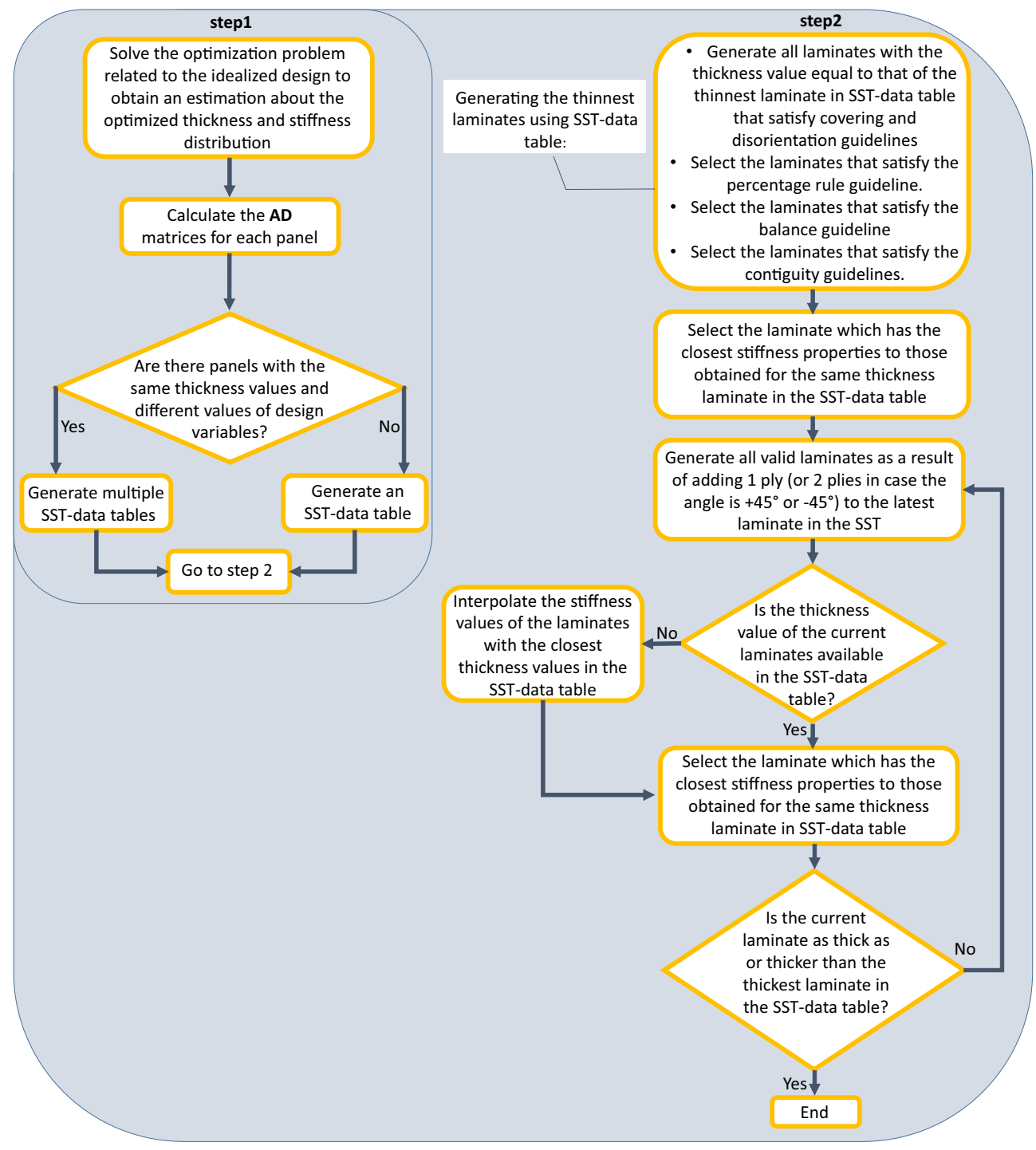

Figure 1: The flowchart of the 2-step procedure of generating an SST. 


\begin{tabular}{|c|c|c|c|c|c|c|c|c|c|c|}
\hline $\begin{array}{c}\text { Number } \\
\text { of plies }\end{array}$ & 32 & 30 & 26 & 24 & 22 & 18 & 16 & 14 & 10 & 8 \\
\hline \multicolumn{11}{|l|}{ ply index } \\
\hline 1 & 45 & 45 & 45 & 45 & 45 & 45 & 45 & 45 & 45 & 45 \\
\hline 2 & 90 & 90 & 90 & 90 & 90 & 90 & 90 & 90 & 90 & 90 \\
\hline 3 & -45 & -45 & -45 & -45 & -45 & & & & & \\
\hline 4 & -45 & -45 & -45 & -45 & -45 & -45 & -45 & -45 & & \\
\hline 5 & 90 & 90 & 90 & 90 & 90 & 90 & & & & \\
\hline 6 & -45 & -45 & & & & & & & & \\
\hline 7 & 90 & & & & & & & & & \\
\hline 8 & -45 & -45 & -45 & -45 & -45 & -45 & -45 & -45 & -45 & -45 \\
\hline 9 & 0 & 0 & 0 & 0 & 0 & 0 & 0 & 0 & 0 & 0 \\
\hline 10 & 0 & 0 & 0 & 0 & & & & & & \\
\hline 11 & 45 & 45 & 45 & 45 & 45 & 45 & 45 & 45 & & \\
\hline 12 & 0 & 0 & 0 & 0 & 0 & 0 & 0 & & & \\
\hline 13 & 0 & 0 & 0 & 0 & 0 & 0 & 0 & 0 & 0 & \\
\hline 14 & 45 & 45 & & & & & & & & \\
\hline 15 & 45 & 45 & 45 & 45 & 45 & & & & & \\
\hline 16 & 0 & 0 & 0 & & & & & & & \\
\hline
\end{tabular}

Figure 2: A generated SST using the 2-step method. Fields marked red indicate dropped plies. Due to symmetry, only the stacking sequences of half-laminates are shown. This SST is generated for the second example of Section 4.

where LV represents the set of all ply stack values in the SST. According to Equation (7) the highest ply stack value below the level-set function at point $X$ gives the number of plies of the laminate covering point $X$. Figure 3 shows a level-set function covering a $1 \mathrm{D}$ structure with length $L$, and the resulting thickness distribution where the ply stack thicknesses are selected from the SST in Figure 2. According to the SST, LV is: $\{8,10,14, \ldots, 32\}$ plies.

Each finite element node will be assigned a level-set function value based on interpolation from the design node values. Within an element the interpolation of the value of the element nodes specifies the level-set function. Figure 4 schematically shows this procedure in three steps. The element obtains area weighted stiffness data based on the area that each stacking sequence covers in the element.

Using the introduced method, the discrete nature of the SST-based optimization problem is turned into a continuous one as the design variables change continuously. The "max" function used in Equation (7) determines the location of the ply drop. It is the continuous change of the location of the ply drop with respect to the (continuous) change of a design variable which is differentiable and gives sensitivities. Figure 5 schematically shows the continuous change of the ply drop location (thickness distribution) as a result of continuous change of design variables. Continuous change of the design variables allows using any linear or quadratic programming algorithm to solve the optimization problem. In the present study, the 'constrained steepest-descent' algorithm [52] is used. Figure 6 schematically shows the optimization framework. 


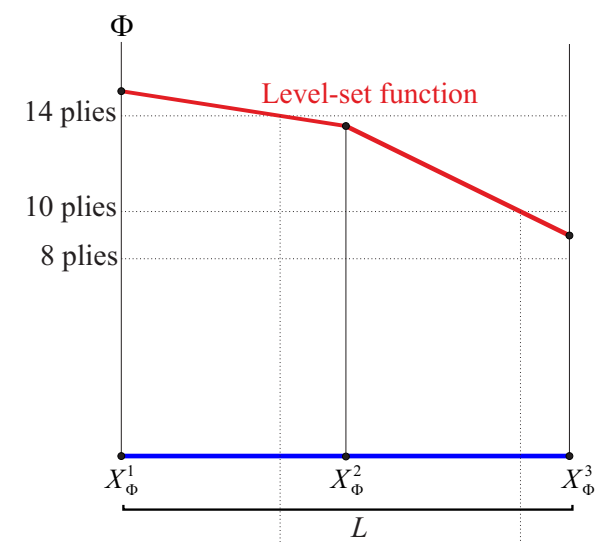

(a) Level-set function covering a 1D structure.

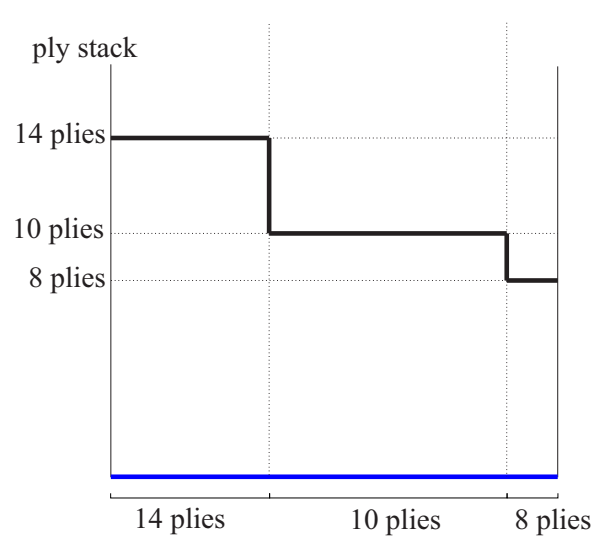

(b) Thickness distribution specified by the level-set function.

Figure 3: Determining thickness distribution using a level-set function

\subsection{Optimization objective}

In the present study, the optimization objective is mass minimization. As the level-set function specifies the thickness distribution all over the structure, it can be concluded that the structure's mass and stiffness can be implicitly derived with a given level-set function by determining the number of plies covering different regions of the structure as discussed earlier. Moreover, since the ply thickness is directly related to the value of the level-set function, a good approximation of the mass $W$ can be obtained by integration of the level-set function over the structural domain $\Omega$ :

$$
W \approx \rho \int \Phi(X) \mathrm{d} \Omega=\rho \sum_{n=1}^{N_{\phi}} A^{n} \Phi^{n}
$$

where $\rho$ is the ply density and $A^{n}$ is connected to the area belonging to design node $n$. This means that the objective function is taken as linear in the design variables.

\subsection{Constraint definition}

In this section we discuss how to minimize the structural mass while subjected to local buckling constraints. The following eigenvalue equation gives the buckling loads $[53]$ :

$$
\left(\mathbf{K}_{\mathrm{B}}-\lambda_{i} \mathbf{K}_{\mathrm{G}}\right) \cdot w_{i}=0
$$

where $\mathbf{K}_{\mathrm{B}}$ is the global bending stiffness matrix, $\mathbf{K}_{\mathrm{G}}$ is the global stress stiffness matrix, and the eigenvalue $\lambda_{i}$ is the $i^{\text {th }}$ load multiplier or the buckling factor. The solution of Equation (9) gives a number of eigenvalues $\lambda$ equal to the rank of $\mathbf{K}_{\mathrm{G}}$. The vector $w_{i}$ is the mode shape corresponding to the $i^{\text {th }}$ buckling factor. The constraints 


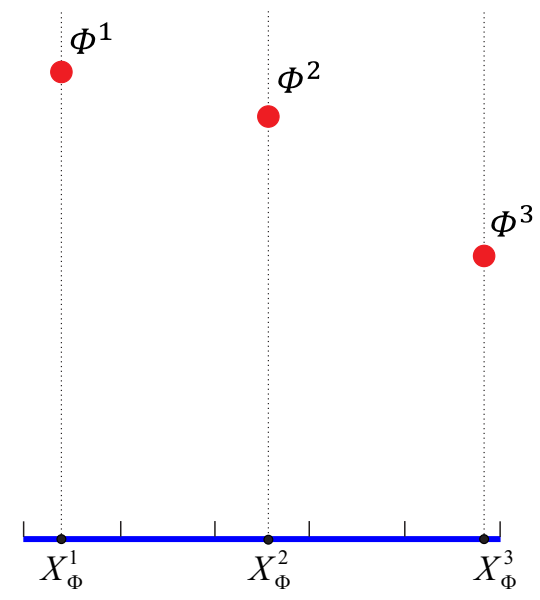

(a) Step 1: only the design nodes $X_{\phi}^{i}$ have level-set function values. These values are indicated by red dots.

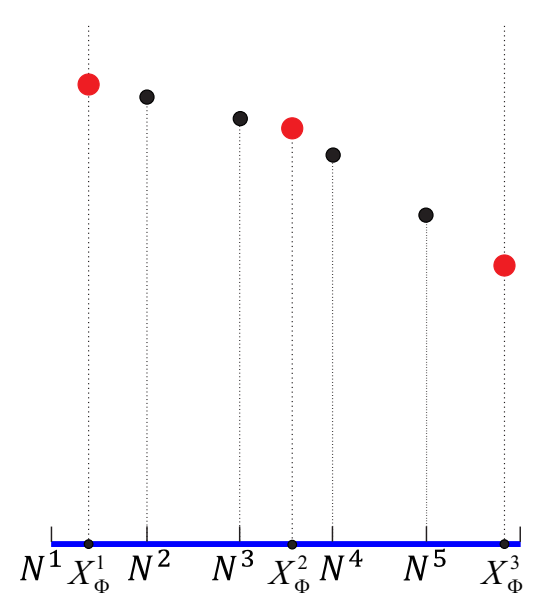

(b) Step 2: each finite element node $N^{i}$ is assigned a level-set function value by interpolating the design node values.

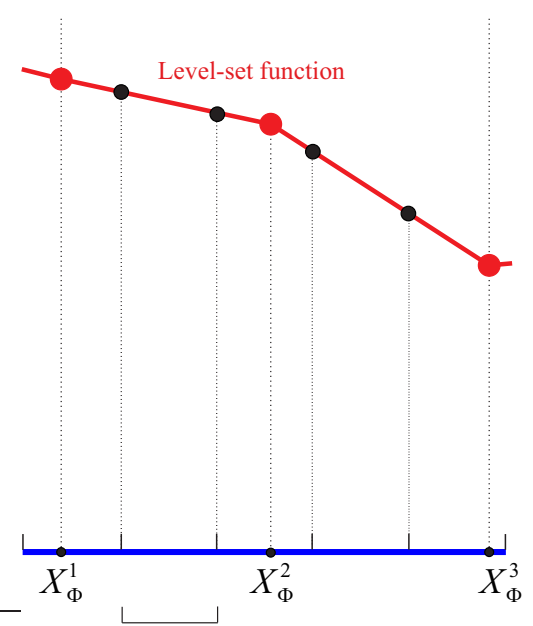

A finite element

(c) Step 3: the level-set function within an element is defined by interpolating the element nodal values.

Figure 4: A 3-step interpolation procedure towards defining a level-set function given the design node values. 


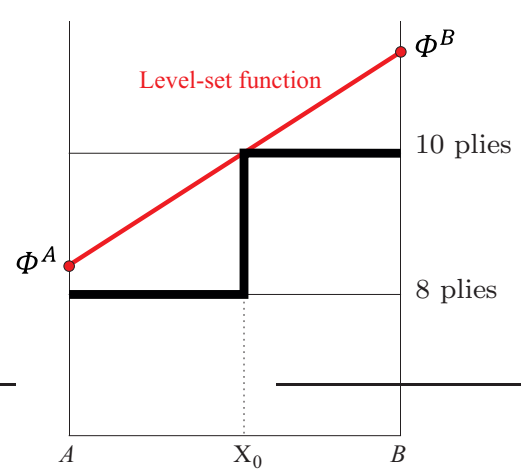

(a) The $\Phi^{A} \Phi^{B}$ line represents a level-set function. The resulting thickness distribution is shown with the black solid line. $\mathrm{X}_{0}$ indicates the ply drop location.

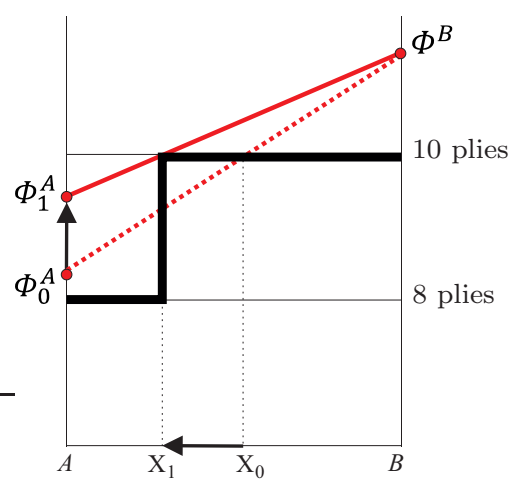

(b) As a result of the continuous change of the design variable $\Phi_{0}^{A}$ towards $\Phi_{1}^{A}$, the ply drop location changes continuously from $\mathrm{X}_{0}$ towards $\mathrm{X}_{1}$.

Figure 5: Continuous change of the thickness distribution as a result of continuous change of design variables.

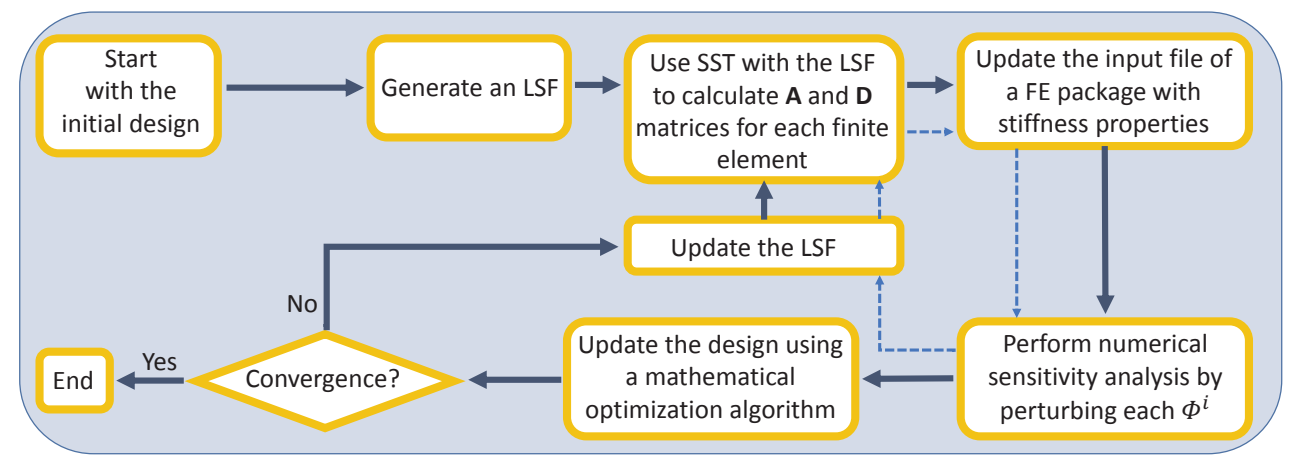

Figure 6: Flowchart of the optimization procedure. The dashed line represents the internal loop for the numerical sensitivity analysis in each iteration of the optimization problem. 
of the problem are defined as [12]:

$$
g_{i}: 1-\lambda_{i} \leq 0 \quad i=1 \text { to } N_{\mathrm{c}}
$$

\subsection{Sensitivity analysis}

As the objective function (Equation (8)) is a linear function of design variables, the sensitivity of the objective function with respect to the design variable $\Phi^{i}$ can be simply obtained through:

$$
\frac{\mathrm{d} W}{\mathrm{~d} \Phi^{i}}=\rho A^{i}
$$

To obtain the sensitivity of constraints, numerical forward difference scheme is used. At a given design, there is a set of $N_{\mathrm{c}}$ buckling factors. Each corresponds to a constraint. The sensitivity of a buckling constraint in (3) can be translated to the sensitivity of the buckling factors. As a result of perturbing a design variable, a new set of $N_{\mathrm{c}}$ buckling factors will be obtained through a finite element analysis. The sensitivity of each buckling factor $\lambda_{i}$ to the $j^{\text {th }}$ design variable is calculated through:

$$
\frac{\mathrm{d} \lambda_{i}}{\mathrm{~d} \Phi^{j}}=\frac{\lambda_{i}\left(\Phi+\delta \Phi^{j}\right)-\lambda_{i}(\Phi)}{\delta \Phi^{j}}
$$

\subsection{Switching of the mode shapes}

The order of the buckling mode shapes may change as the design of the structure changes. During the numerical sensitivity analysis, it is crucial that in (12) the two buckling factors being subtracted from each other, belong to the same mode shape. Therefore, when a new set of buckling factors is obtained after perturbing a design variable, their corresponding mode shapes must be extracted too. As mentioned earlier, eigenvectors in (3) represent the buckling mode shapes corresponding to each buckling factor. The eigenvectors include the values of the out of plane translational and rotational degrees of freedom. For two eigenvectors $w_{i}$ and $w_{j}$, obtained from two different buckling analyses, we can calculate $\mathrm{MAC}_{i j}=\frac{\left|w_{i}^{T} \cdot w_{j}\right|}{\left|w_{i}\right|\left|w_{j}\right|}$. If $\mathrm{MAC}_{i j} \approx 1$, the eigenvectors are correlated; otherwise, $w_{i}$ and $w_{j}$ represent different mode shapes. Detailed information on tracking mode shapes can be found in [54].

In the present study, the linearized 'constrained steepest-descent' algorithm [52] is used to solve the optimization problem. If, alternatively, a non-linear programming algorithm (e.g. Sequential Quadratic Programming (SQP) [52]) is used, the mode shapes also have to be tracked between the iterations of the optimization problem. The reason is that the second derivative information is required in these algorithms. The second derivative information is usually approximated using the first derivative information of the iterations of the optimization problem [52]. Therefore, it is crucial to make sure that the first derivative information of the buckling constraints also belong to the same mode shape.

A linearized optimization algorithm (although less efficient compared to e.g. an SQP algorithm) does not require the second derivative information and thus is selected for the present study. 


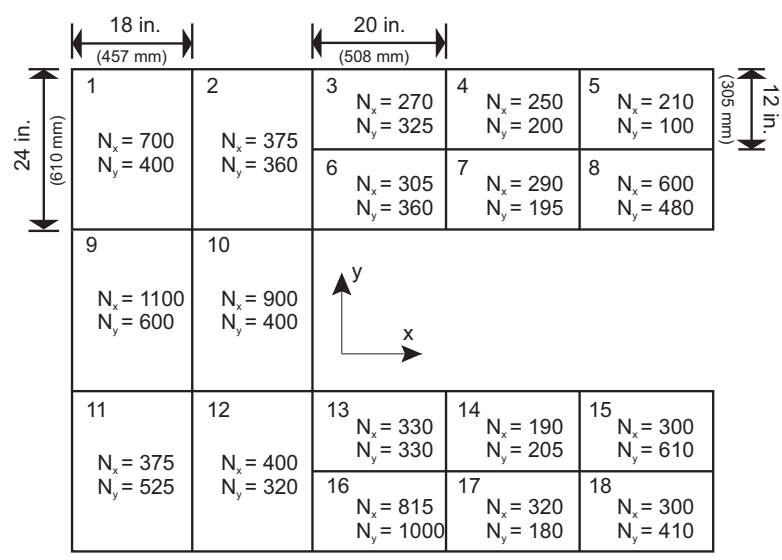

Figure 7: 18-panel horseshoe configuration, all loads in lbf/in $(\times 175.1$ for $\mathrm{N} / \mathrm{m})$

\subsection{Mesh density}

A reliable buckling analysis requires sufficient density of the finite element mesh. To obtain a proper element size, a target structure has to be subjected to buckling analysis, each time with a finer mesh size until the difference in the critical buckling factor as the result of remeshing is negligible. A mesh study is performed for the examples presented in Section 4.

\section{Results and discussion}

In this section, two examples are presented to show the performance of the proposed level-set method. In the first example it is applied to the well-known 18-panel problem in a horseshoe configuration as shown in Figure 7. This problem was first proposed by Soremekun et al. [11] and subsequently studied in [1, 7, 17, 34-37].

In the Horseshoe Problem, the load redistribution is ignored. This results in a too simplified problem. To examine the capability of the method in dealing with real problems, in the second example the proposed method is applied to the optimization of a multi-panel composite skin of a torsion box.

Four-node, quadrilateral shell elements with 6 degrees of freedom per node are used for the finite element analysis in both examples. As mentioned earlier, to form the level set function $\Phi$, as defined in Equation (6), bilinear interpolation functions are used.

\subsection{Example 1, Horseshoe Problem}

The optimization objective in this problem is mass minimization of the whole structure without individual panel failure under buckling. The final solution in this problem has 


\begin{tabular}{|c|c|c|c|c|c|c|c|c|c|c|c|c|c|c|c|c|c|c|}
\hline & 48 & 46 & 44 & 42 & 40 & 36 & 34 & 32 & 30 & 28 & 24 & 22 & 20 & 18 & 16 & 12 & 10 & 8 \\
\hline \multicolumn{19}{|c|}{ ply index } \\
\hline 1 & 45 & 45 & 45 & 45 & 45 & 45 & 45 & 45 & 45 & 45 & 45 & 45 & 45 & 45 & 45 & 45 & 45 & 45 \\
\hline 2 & 90 & 90 & 90 & 90 & 90 & 90 & 90 & 90 & 90 & 90 & 90 & 90 & 90 & 90 & 90 & 90 & 90 & 90 \\
\hline 3 & 90 & & & & & & & & & & & & & & & & & \\
\hline 4 & -45 & -45 & -45 & -45 & -45 & & & & & & & & & & & & & \\
\hline 5 & 90 & 90 & 90 & 90 & 90 & 90 & & & & & & & & & & & & \\
\hline 6 & 90 & 90 & & & & & & & & & & & & & & & & \\
\hline 7 & 45 & 45 & 45 & 45 & 45 & 45 & 45 & 45 & 45 & 45 & & & & & & & & \\
\hline 8 & 0 & 0 & 0 & 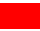 & & & & & & & & & & & & & & \\
\hline 9 & 0 & 0 & 0 & 0 & & & & & & & & & & & & & & \\
\hline 10 & 45 & 45 & 45 & 45 & 45 & & & & & & & & & & & & & \\
\hline 11 & 90 & 90 & 90 & 90 & 90 & 90 & 90 & & & & & & & & & & & \\
\hline 12 & 90 & 90 & 90 & 90 & 90 & 90 & 90 & 90 & 90 & 90 & 90 & 90 & 90 & 90 & 90 & 90 & 90 & \\
\hline 13 & -45 & -45 & -45 & -45 & -45 & -45 & -45 & -45 & -45 & -45 & & & & & & & & \\
\hline 14 & 0 & 0 & 0 & 0 & 0 & 0 & 0 & 0 & & & & & & & & & & \\
\hline 15 & 0 & 0 & 0 & 0 & 0 & 0 & 0 & 0 & 0 & & & & & & & & & \\
\hline 16 & -45 & -45 & -45 & -45 & -45 & -45 & -45 & -45 & -45 & -45 & -45 & -45 & -45 & -45 & -45 & & & \\
\hline 17 & 90 & 90 & 90 & 90 & 90 & 90 & 90 & 90 & 90 & 90 & 90 & 90 & 90 & & & & & \\
\hline 18 & 90 & 90 & 90 & 90 & 90 & 90 & 90 & 90 & 90 & 90 & 90 & 90 & 90 & 90 & & & & \\
\hline 19 & -45 & -45 & -45 & -45 & -45 & -45 & -45 & -45 & -45 & -45 & -45 & -45 & -45 & -45 & -45 & -45 & -45 & -45 \\
\hline 20 & 0 & 0 & 0 & 0 & 0 & 0 & 0 & 0 & 0 & 0 & 0 & 0 & 0 & 0 & 0 & 0 & 0 & 0 \\
\hline 21 & 0 & 0 & 0 & 0 & 0 & 0 & 0 & 0 & 0 & 0 & 0 & 0 & 0 & 0 & 0 & 0 & & \\
\hline 22 & 45 & 45 & 45 & 45 & 45 & 45 & 45 & 45 & 45 & 45 & 45 & 45 & 45 & 45 & 45 & & & \\
\hline 23 & 90 & 90 & 90 & 90 & 90 & 90 & 90 & 90 & 90 & 90 & 90 & & & & & & & \\
\hline 24 & 90 & 90 & 90 & 90 & 90 & 90 & 90 & 90 & 90 & 90 & 90 & 90 & & & & & & \\
\hline
\end{tabular}

Figure 8: The SST generated for the Horseshoe Problem.

to be blended. Here, the formerly mentioned set of $\left\{0^{\circ}, \pm 45^{\circ}\right.$, and $\left.90^{\circ}\right\}$ is used as fiber orientations. For the construction of each ply a Graphite/Epoxy IM7/8552 material is used where $E_{1}=141 \mathrm{GPa}(20.5 \mathrm{MSi}), E_{2}=9.03 \mathrm{GPa}(1.31 \mathrm{MSi}), G_{12}=4.27 \mathrm{GPa}$ (0.62 MSi), $\nu_{12}=0.32$, and ply thickness is $0.191 \mathrm{~mm}$ (0.0075 in.). The optimization problem is formulated as follows:

$$
\begin{aligned}
\min & W\left(\Phi^{N}\right) \\
\text { subject to } & g_{i j} \leq 0 \quad i=1 \text { to } 5 \quad \text { and } \quad j=1 \text { to } 18 \\
& \Phi_{\min } \leq \Phi^{n} \leq \Phi_{\max }
\end{aligned}
$$

where $W$ represents the overall mass of the structure as a function of design node vector $\Phi^{N}$. $\Phi_{\min }$ and $\Phi_{\max }$ denote lower bound and upper bound for the values at the design nodes, respectively. $\Phi_{\min }$ and $\Phi_{\max }$ has to be determined based on the idealized design. $g_{i j}$ represents a constraint on the $i^{\text {th }}$ buckling factor of the $j^{\text {th }}$ panel. As it can be seen in Equation (13), five mode shapes are considered as buckling constraints for each panel. Thus the total number of 90 buckling constraints is imposed to this problem.

The SST shown in Figure 8 is used for the thickness optimization of the Horseshoe Problem. A detailed description on generating this SST, as an example, is provided in Appendix A.

For the optimization of the thickness distribution, 12 design nodes are distributed as shown in Figure 9. As each panel may have a varying thickness, finite element analysis is performed to calculate the buckling factors for each panel. To have a valid mesh size, a mesh study, as described in Section 3.6, is performed on a typical panel. Based on the mesh study, shell elements with mesh size of $0.0254 \times 0.0254 \mathrm{~m}(1 \times 1 \mathrm{in}$. $)$ are adopted for the simulations. According to the generated SST, shown in Figure 8, 


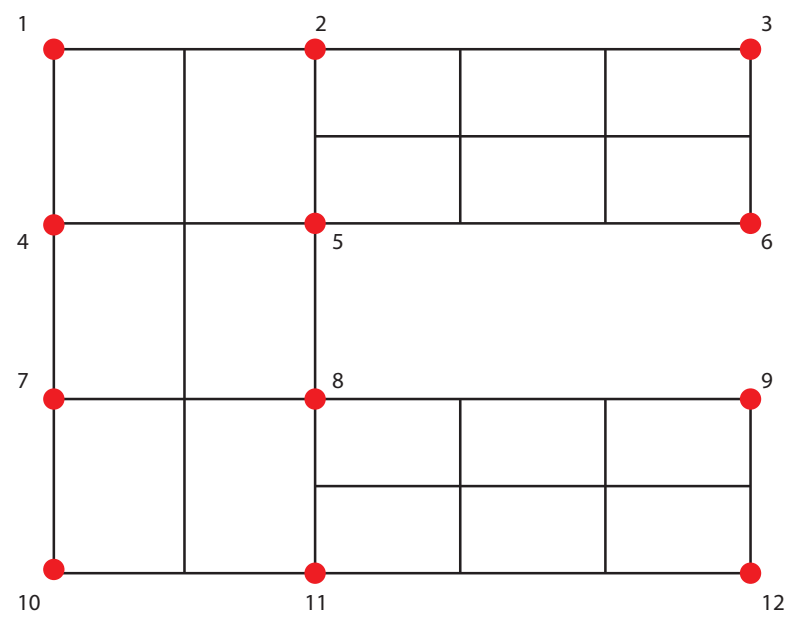

Figure 9: The distribution and the numbering of 12 design nodes.

Table 1: The initial design used for the level-set-based thickness optimization for the Horseshoe Problem. The distribution of the design variables is shown in Figure 9

\begin{tabular}{ccccccccccccc}
\hline Design variable index & 1 & 2 & 3 & 4 & 5 & 6 & 7 & 8 & 9 & 10 & 11 & 12 \\
Initial value (plies) & 38 & 25 & 20 & 44 & 40 & 26 & 42 & 38 & 28 & 35 & 32 & 24 \\
\hline
\end{tabular}

the thickness value of the thickest laminate is $0.191 \times 48=9.17 \mathrm{~mm}$. As this value is small compared to the smallest panel dimension $(305 \mathrm{~mm})$, laminates are considered to be thin and the kinematics of Kirchhoff theory is used for the shell elements. The entire Horseshoe Problem is discretized into 5472 shell elements and the total number of 5701 nodes.

As described before, the values of the design variables prescribe a thickness distribution over the structure. As for the initial design, it is reasonable to assign values to design variables such that the resulting thickness distribution is close to the idealized thickness distribution. Table 1 shows the initial design used for the level-set-based thickness optimization (refer to Appendix A for the idealized design information related to the Horseshoe Problem).

Figure 10 shows the contour plot of thickness distributions at the point of optimum. The optimization problem converges in 4 iterations where each iteration takes about 15 min on a regular PC (CPU: $2.6 \mathrm{GHz}, \mathrm{RAM}: 8 \mathrm{~GB})$.

Table 2 shows the average number of plies in each panel. As the thickness distribution varies over the surface of each panel, to show the final result, an average thickness is used to have an indication of the number of plies in each panel. The overall weight of the structure in this case is $32.64 \mathrm{~kg}$. Buckling factors obtained for each panel at the point of optimum are also shown in Table 2. As it can be seen in the table, panels $6,7,13$, and 14 have eigenvalues much higher than their critical value. The 


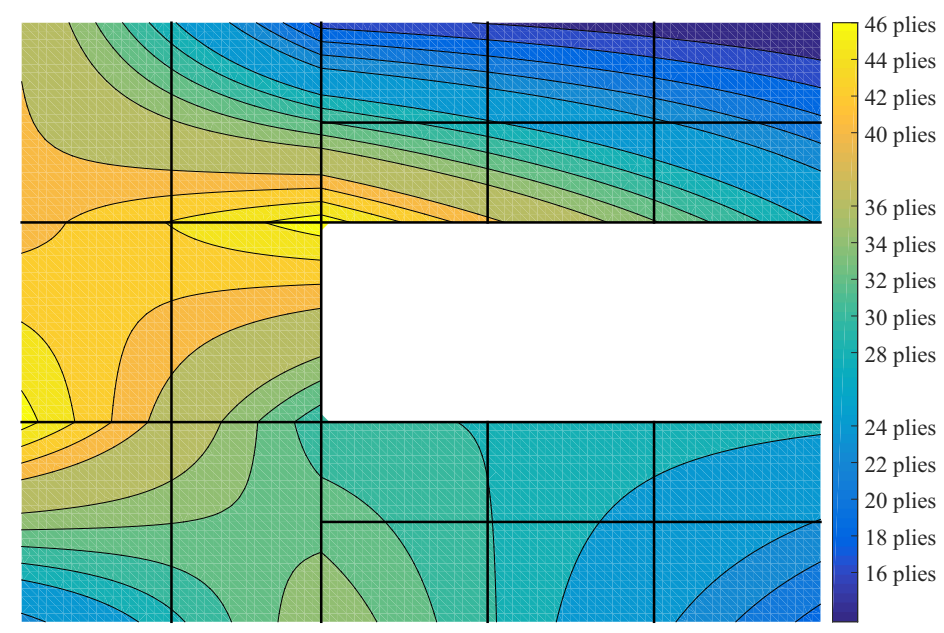

Figure 10: Contour plot of the thickness distribution for the optimization problem with 12 design nodes.

reason is that the number of design nodes shown in Figure 9 is not sufficient to prescribe thickness distributions such that all panels become critical with respect to buckling. For example, the thicknesses of panels 13-18 are all interpolated through only 4 design nodes (design nodes 8, 9, 11, and 12 in Figure 9). As panels 15 and 16 have eigenvalues very close to their critical value, the mentioned design nodes do not allow for thinner laminates. Therefore, some panels inevitably remain thicker than their potential optimum thickness.

To overcome this, more design nodes are added as shown in Figure 11. As it can be seen in the figure, the design nodes do not have to be placed on panels corners. The design nodes can be placed wherever a more detailed design is required. Figure 12 shows the contour plot of the optimized thickness distribution with 30 design nodes. The same trend of thickness distribution (left side of the horseshoe with thicker laminates than the right side) shown in Figure 10 is observed in Figure 12 as well. However, due to the increased number of design nodes, the ply drop locations are more freely prescribed over the surface of each panel.

It is interesting to mention that the optimum design of the problem with 12 design nodes is used as the initial design of the optimization problem with 30 design nodes. As the initial design is already reasonably close to the optimum point, it takes only 4 iterations until the optimization problem with 30 design nodes converged. The final mass of the structure in this case is $30.60 \mathrm{~kg}$. Compared to the problem with 12 design nodes, a further reduction of $2.04 \mathrm{~kg}$ in mass is obtained by increasing the number of design nodes. As indicated by the results, a lighter design may require more design nodes. A problem with more design nodes is computationally more 
Table 2: Average number of plies and the obtained buckling factors for each panel at the optimum design with 12 design nodes.

\begin{tabular}{ccc}
\hline Panel & Buckling factor & Average number of plies \\
\hline 1 & 1.035 & 36.73 \\
2 & 1.060 & 32.87 \\
3 & 1.141 & 22.08 \\
4 & 1.110 & 19.53 \\
5 & 1.037 & 16.77 \\
6 & 4.064 & 35.76 \\
7 & 3.860 & 30.54 \\
8 & 1.000 & 25.23 \\
9 & 1.034 & 41.75 \\
10 & 1.000 & 38.74 \\
11 & 1.012 & 33.13 \\
12 & 1.211 & 32.54 \\
13 & 2.661 & 30.13 \\
14 & 3.625 & 27.67 \\
15 & 1.007 & 25.14 \\
16 & 1.004 & 31.40 \\
17 & 2.456 & 26.16 \\
18 & 1.028 & 22.57 \\
\hline
\end{tabular}

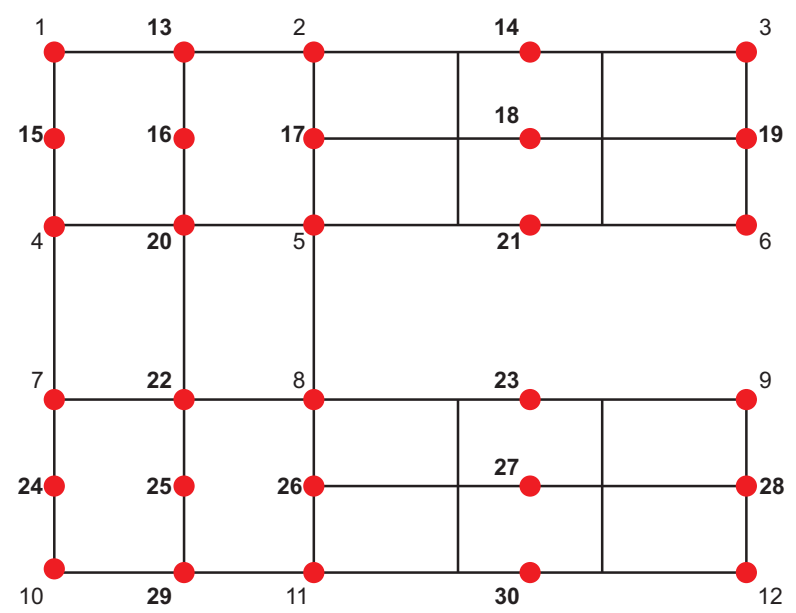

Figure 11: The distribution and the numbering of 30 design nodes. The bold labels are related to the design nodes added to the configuration shown in Figure 9. 


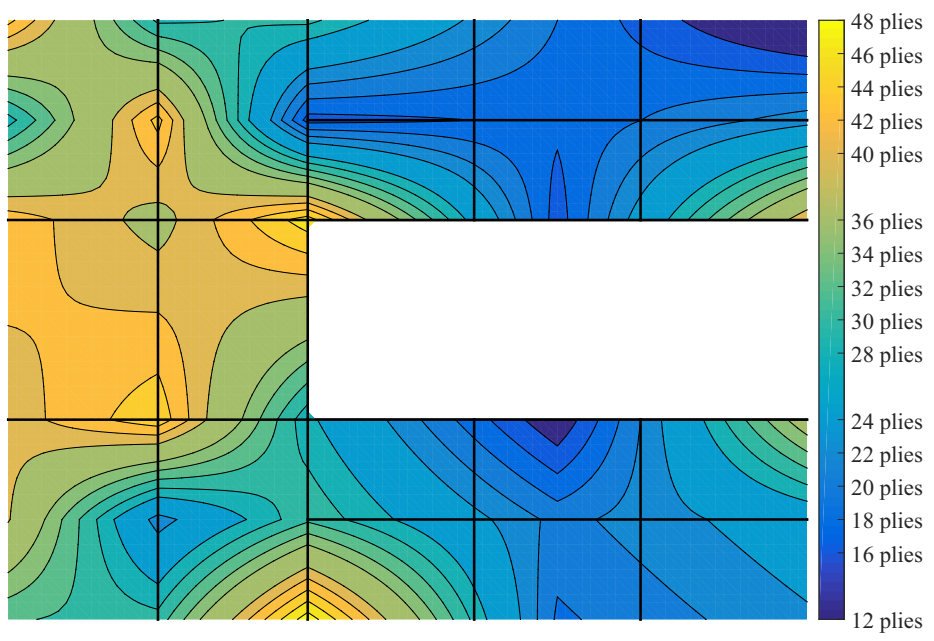

Figure 12: Contour plot of the thickness distribution for the optimization problem with 30 design nodes.

expensive because of more required sensitivity calculations. However, here it is shown that the optimization problem can start with a reasonably small number of design nodes until the initial design is improved towards the optimum point in that design space. Then design nodes can be added locally wherever a more detailed design is required. As a result, only a small part of the optimization problem proceeds with a relatively high number of design nodes. This reduces the overall computation time of the optimization problem. Figure 13 shows the mass evolution for the optimization of both 12 and 30 design nodes in a single graph.

Table 3 shows the average ply number and the buckling factor of each panel after optimization with 30 design nodes. The average number of plies in each panel is compared to those reported in [1]. As it can be seen in Table 3, the obtained ply number for each panel is in a good agreement with those reported in literature.

The final weight of the structure obtained using the proposed method is higher than that reported in $[1,35]$. This is due to two reasons. Firstly, in $[1,35]$ there are more options for ply orientations than used in the current study. More options for ply orientations result in a more optimal placement of fibers which may finally cause a lower number of plies to provide sufficient stiffness in a specific region of the structure. Secondly, the reported results in $[1,35]$ are only valid for symmetric and balanced laminates while the presented results in the current study are valid for all design guidelines of laminates mentioned in Section 2. Naturally, as more design guidelines are added to the optimization problem, a heavier feasible design is obtained. For example, in [1] the reported mass for only symmetric laminate is $28.44 \mathrm{~kg}$. But, this value increases to 28.82 when balance is added. 


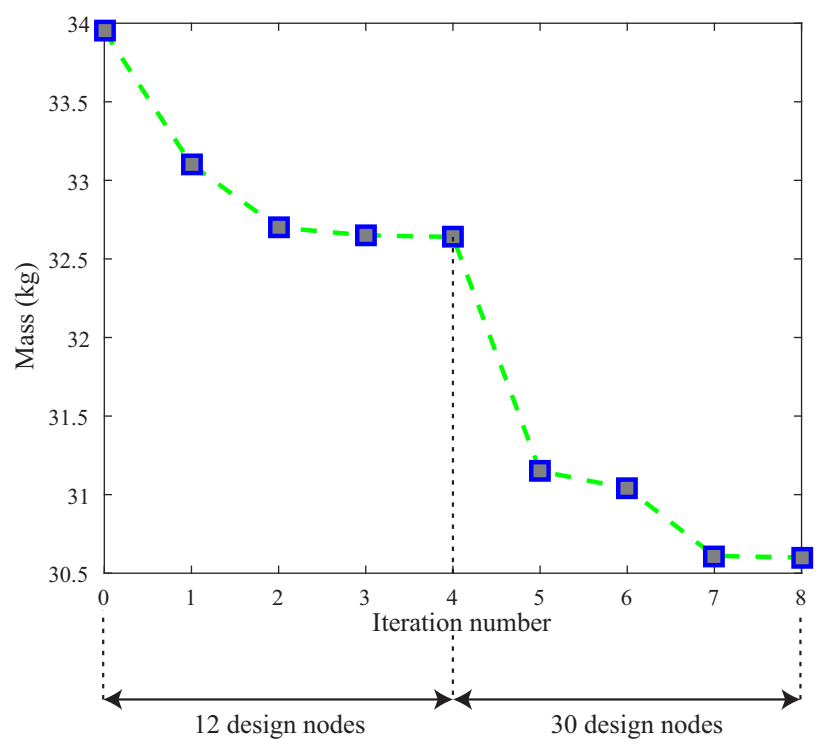

Figure 13: Mass evolution for the optimization of both 12 and 30 design nodes. The final design of the optimization with 12 design nodes is used as the initial design for optimization with 30 design nodes.

Table 3: Average number of plies and the obtained eigenvalues for each panel at the optimum design with 30 design nodes.

\begin{tabular}{cccc}
\hline Panel & Buckling factor & Average number of plies & $\begin{array}{c}\text { Number of plies } \\
\text { Yang et al. [1] }\end{array}$ \\
\hline 1 & 1.015 & 36.61 & 34 \\
2 & 1.006 & 32.43 & 28 \\
3 & 1.033 & 21.14 & 22 \\
4 & 1.001 & 18.48 & 20 \\
5 & 1.092 & 16.82 & 16 \\
6 & 1.448 & 26.04 & 22 \\
7 & 1.014 & 19.18 & 20 \\
8 & 1.000 & 26.21 & 26 \\
9 & 1.007 & 41.22 & 36 \\
10 & 1.002 & 38.71 & 30 \\
11 & 1.011 & 33.12 & 28 \\
12 & 1.017 & 31.61 & 22 \\
13 & 1.413 & 24.45 & 26 \\
14 & 1.006 & 18.82 & 32 \\
15 & 1.004 & 25.70 & 20 \\
16 & 1.009 & 32.39 & 24 \\
18 & 1.378 & 21.35 & \\
\hline
\end{tabular}


Regarding the result comparison, it is important to mention that the results reported in $[1,35]$ are obtained for panels with constant laminate thickness. However, using the proposed method, the laminate thickness can vary in each panel. As the purpose of the result comparison is to investigate the validity of the proposed method, an area weighted average of thicknesses in each panel is presented.

\subsection{Example 2, torsion box skin}

The stiffened skin of a wing torsion box (Figure 14) is the target structure for optimization in this example. The wing torsion box is subjected to a load case, which has been analyzed with a reasonably coarse mesh (Figure 14). From this analysis the free body diagram of the skin has been isolated and the loads applied on it have been extracted. Figure 15 shows the loads applied to the skin. In the figure, arrows parallel to each edge represent shear loads in the direction of the arrow. The skin is simply supported on the right edge and the out of plane translational degree of freedom for all edges and stiffeners is set to be zero. To obtain proper values for local buckling, the finite element model has been modified such that each (slightly curved) panel is modeled as flat. To obtain a proper mesh density for buckling analysis, a panel between two ribs and two stringers was studied as described in Section 3.6. A typical panel is discretized into 22 by 6 elements. The entire structure is discretized into 13311 shell elements with 13624 nodes. ABAQUS 6.12 finite element package is used for analysis. Following the procedure shown in Figure 1, the SST shown in Figure 2 is obtained. According to the SST, the LV set is $\{8,10,14,16, \ldots\}$ plies, where the ply thickness is equal to $0.13 \mathrm{~mm}$. According to the generated SST (shown in Figure 2 ), the thickness value of the thickest laminate is $0.13 \times 32=4.16 \mathrm{~mm}$. As this value is small compared to the smaller dimension of a typical panel (143 mm), laminates are considered to be thin and the kinematics of Kirchhoff theory is used for the shell elements. For each individual element individual values of the $\mathbf{A}$ (extensional stiffness) and the $\mathbf{D}$ (bending stiffness) matrices can be specified according to the classical laminated plate theory. To optimize the structure, 9 nodes have been appointed to be the design nodes of the problem with locations as can be seen in Figure 16. Thirty eigenvalues are considered as constraints of the problem. The initial values of the design variables are given such that the resulting level-set function prescribes a laminate with constant thickness equal to 24 plies all-over the structure. The purpose for this choice is to investigate the capability of the proposed method in thickness optimization when the designer has no clue about the optimized configuration.

Figure 17 shows the contour plot of the thickness distribution of the optimized structure. As it can be seen in the figure, the laminates are thicker in the aft-root region of the structure. The front-root corner of the structure is covered with a relatively thin laminate. This can be expected because according to Figure 15, for this load case the front-root corner of the structure is under less compression thus is less critical for buckling.

In a well-posed optimization problem it is expected to have active constraints not more than the number of design variables. Thirty eigenvalues are considered as constraints 


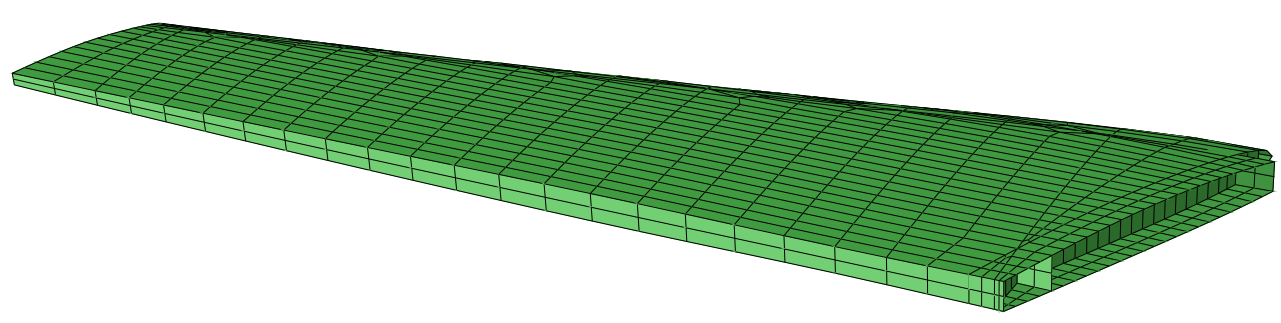

Figure 14: Finite element model of a wing torsion box. The torsion box is subjected to a load case and has been analyzed with a reasonably coarse mesh.

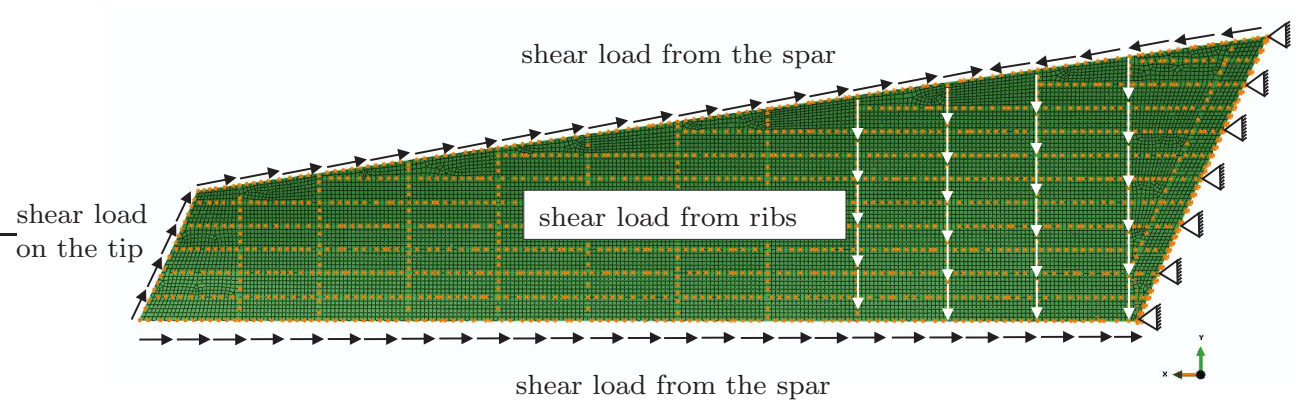

Figure 15: The geometry, loads, and supports of the torsion box skin.

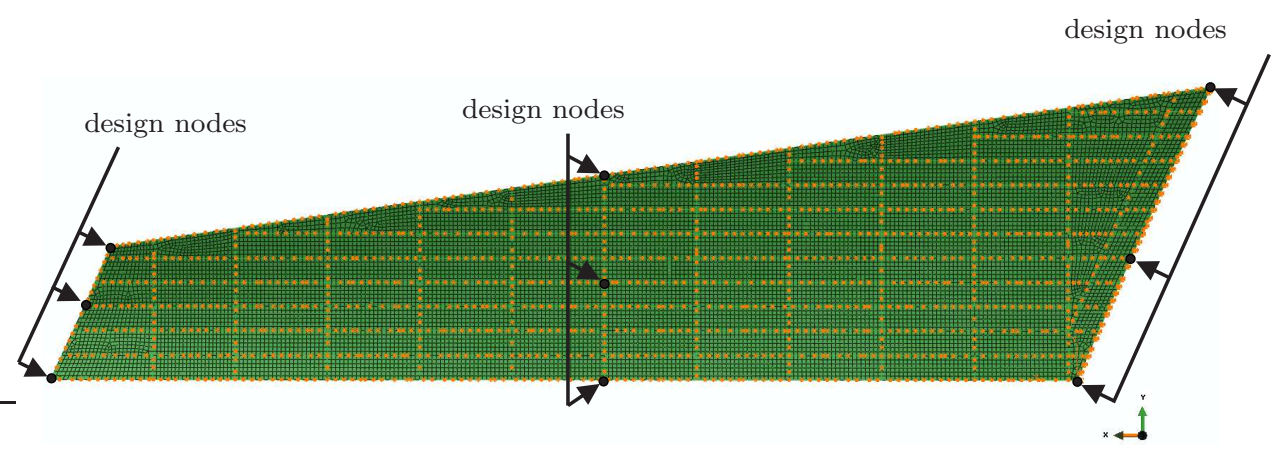

Figure 16: Design nodes' locations are shown with pointed dots. 


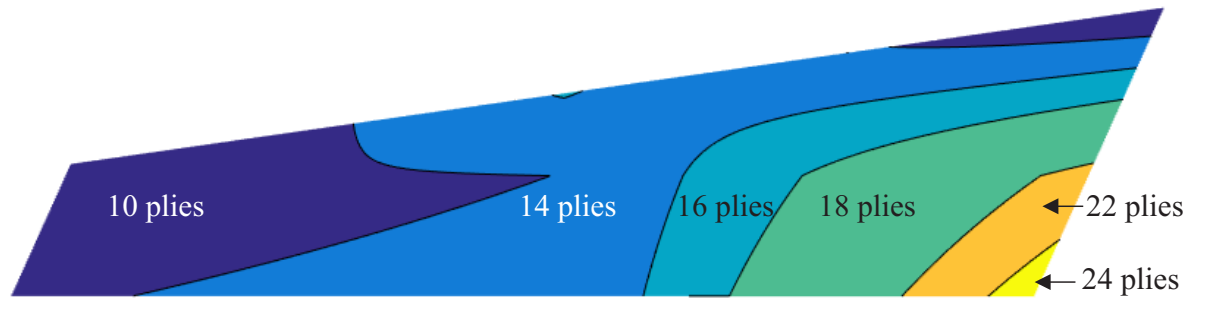

Figure 17: Contour plot of the thickness distribution of the optimized skin, the stacking sequences can be read from the SST in Figure 2.

Table 4: The first 9 eigenvalues of the optimized structure.

\begin{tabular}{ccc}
\hline Eigenvalues & Eigenvalues & Eigenvalues \\
1 to 3 & 4 to 6 & 7 to 9 \\
\hline 1.0043 & 1.0661 & 1.1719 \\
1.0358 & 1.0962 & 1.1897 \\
1.0489 & 1.1360 & 1.2031 \\
\hline
\end{tabular}

of the optimization problem. Table 4 shows the first 9 eigenvalues at the point of optimum.

To show the convergence speed of the algorithm, evolution of the first 30 eigenvalues at each iteration is shown in Figure 18. As the design improves towards the optimum point, the structure becomes more critical with respect to buckling. As it can be seen in Figure 18, it takes only 4 iterations until eigenvalues of the problem become very close to 1 . Figure 19 shows the history of the mass converging to the minimized value. The computation time for each iteration of the optimization process on a regular PC is about 40 minutes and the convergence to the lightest design takes about 450 minutes. Here, the convergence criterion is defined as $\left\|\mathbf{d}^{(k)}\right\| \leq \epsilon_{1}$ and the maximum constraint violation $V_{k} \leq \epsilon_{2}$, where $\left\|\mathbf{d}^{(k)}\right\|$ denotes the norm of the search direction at iteration $k$ and $\epsilon_{1}$ and $\epsilon_{1}$ are two small numbers larger than zero [52].

According to Figure 15, the aft-root corner of the structure is under compression from both the spar and the ribs, thus is covered with the highest number of plies relative to the other regions of the skin. This means that the aft-root corner was critical for buckling before optimization. For the optimum design, however, this region is no longer critical for buckling. This can be verified in Figure 20 where the first five buckling mode shapes together with the $30^{\text {th }}$ one are shown. As it can be seen in the figure, the critical modes occur in regions other than the aft-root corner of the skin.

Only 9 design nodes are used for the design shown in Figure 17. However, as described in example 1 , the proposed method is flexible to add more design nodes to obtain a more detailed design which results in a lower mass. To verify this, 3 more design nodes are added to the optimization problem as can be seen in Figure 21.

Figure 22 shows the contour plot of the thickness distribution of the new optimum 


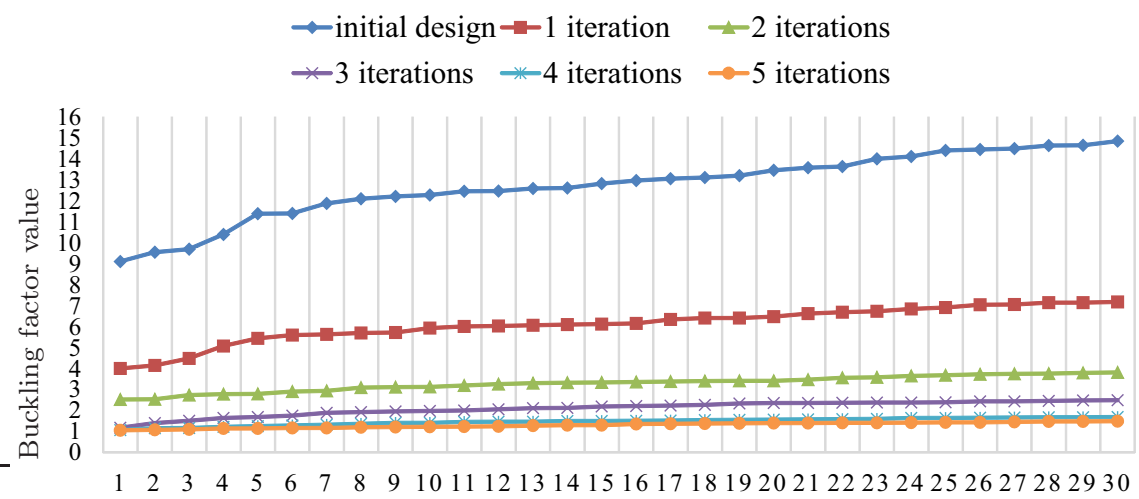

Eigenvalue index

Figure 18: Improvement of the eigenvalues from the initial design towards their critical value at the point of optimum.

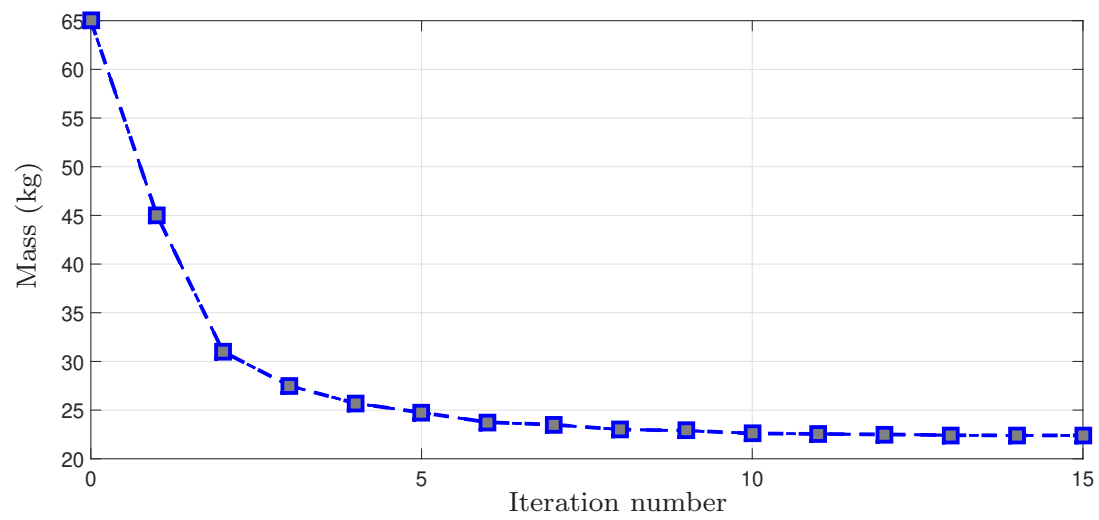

Figure 19: Mass history towards the minimized value. 


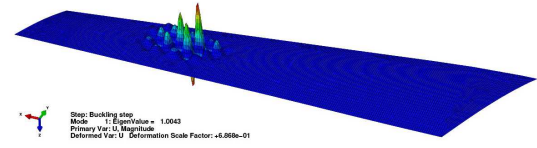

(a) Mode 1

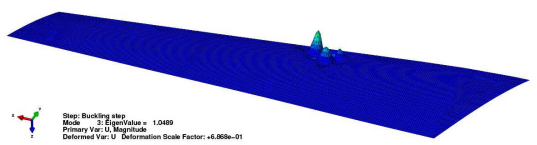

(c) Mode 3

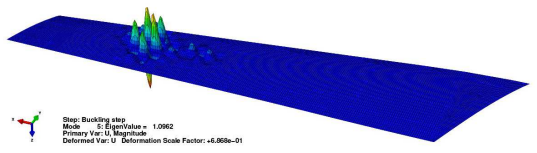

(e) Mode 5

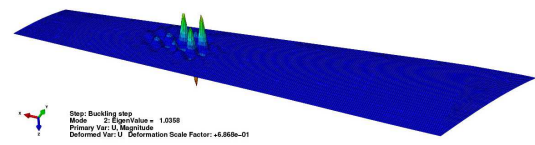

(b) Mode 2

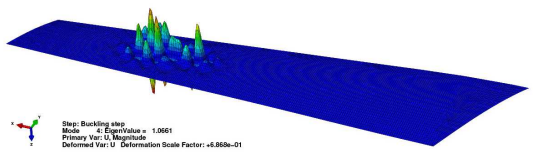

(d) Mode 4

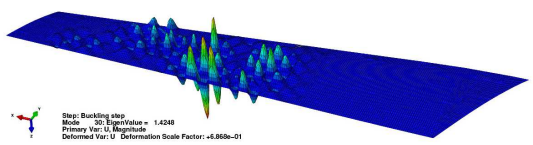

(f) Mode 30

Figure 20: The first five buckling mode shapes together with the $30^{\text {th }}$ one where the structure is covered with the optimized configuration and is subjected to loads as shown in Figure 15.

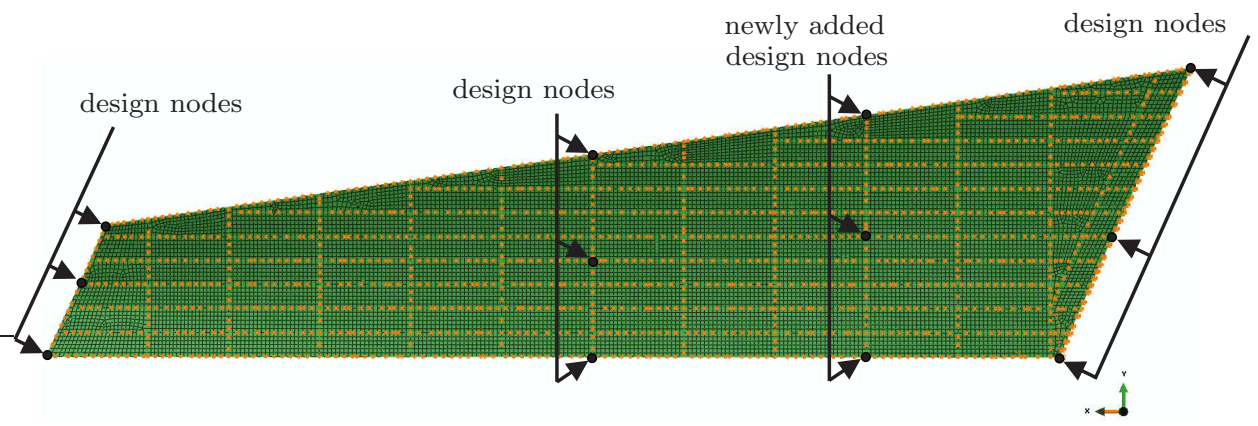

Figure 21: Updated design nodes' locations are shown with pointed dots. 


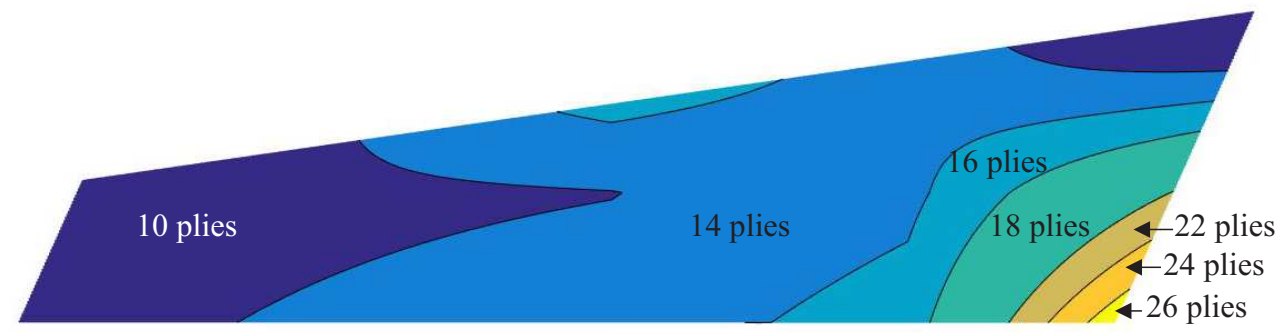

Figure 22: Contour plot of the thickness distribution of the optimized skin using 12 design nodes, the stacking sequences can be read from the SST in Figure 2.

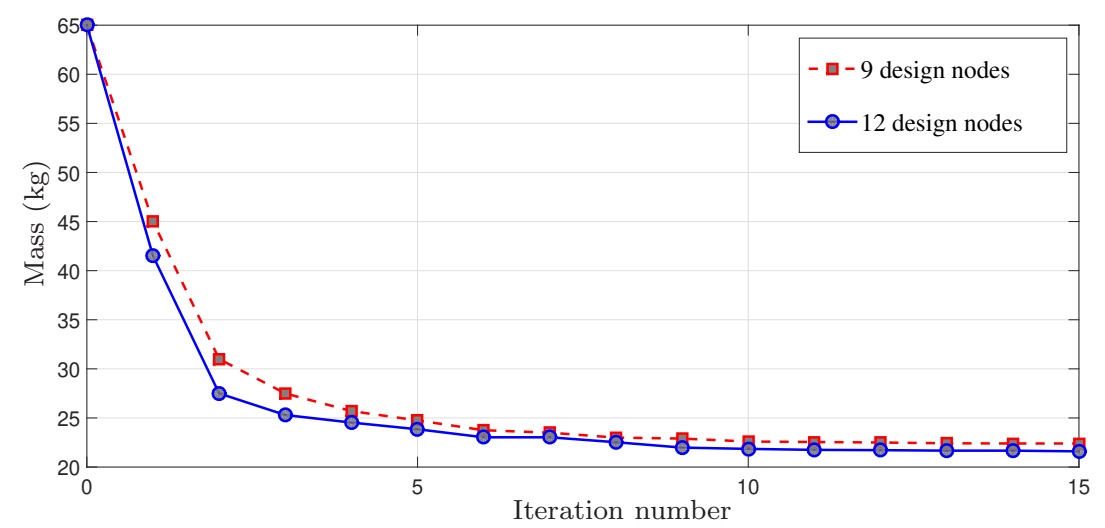

Figure 23: Mass history comparison of the optimization using 9 design nodes with that using 12 design nodes.

design. As more design nodes are added, the optimization program has more freedom to minimize the mass while local buckling is prevented. This can be seen in Figure 23 where the mass histories of the optimizations with 9 and 12 design nodes are compared.

As described in example 1, an interesting feature of the proposed method is that the optimization can start with reasonably few design nodes and continue until a design close to the optimum is reached. Then, the design obtained with few design nodes is used as the initial design of the optimization problem with more design nodes to obtain a more detailed design resulting in a lower mass. This can be seen in Figure 24 where the design with 9 design nodes (Figure 16) after 5 iterations is used as the initial design of the optimization with 3 additional design nodes (Figure 21). Table 5 shows the first 12 eigenvalues at the point of optimum.

As the optimization can partly proceed using less design nodes, the overall convergence time is reduced compared to the case where the optimization starts with 12 design nodes. 
Table 5: The first 12 eigenvalues of the optimized structure with 12 design nodes.

\begin{tabular}{ccc}
\hline Eigenvalues & Eigenvalues & Eigenvalues \\
1 to 4 & 5 to 8 & 9 to 12 \\
\hline 1.0000 & 1.0791 & 1.1224 \\
1.0096 & 1.0848 & 1.1235 \\
1.0554 & 1.0960 & 1.1314 \\
1.0709 & 1.0963 & 1.1384 \\
\hline
\end{tabular}

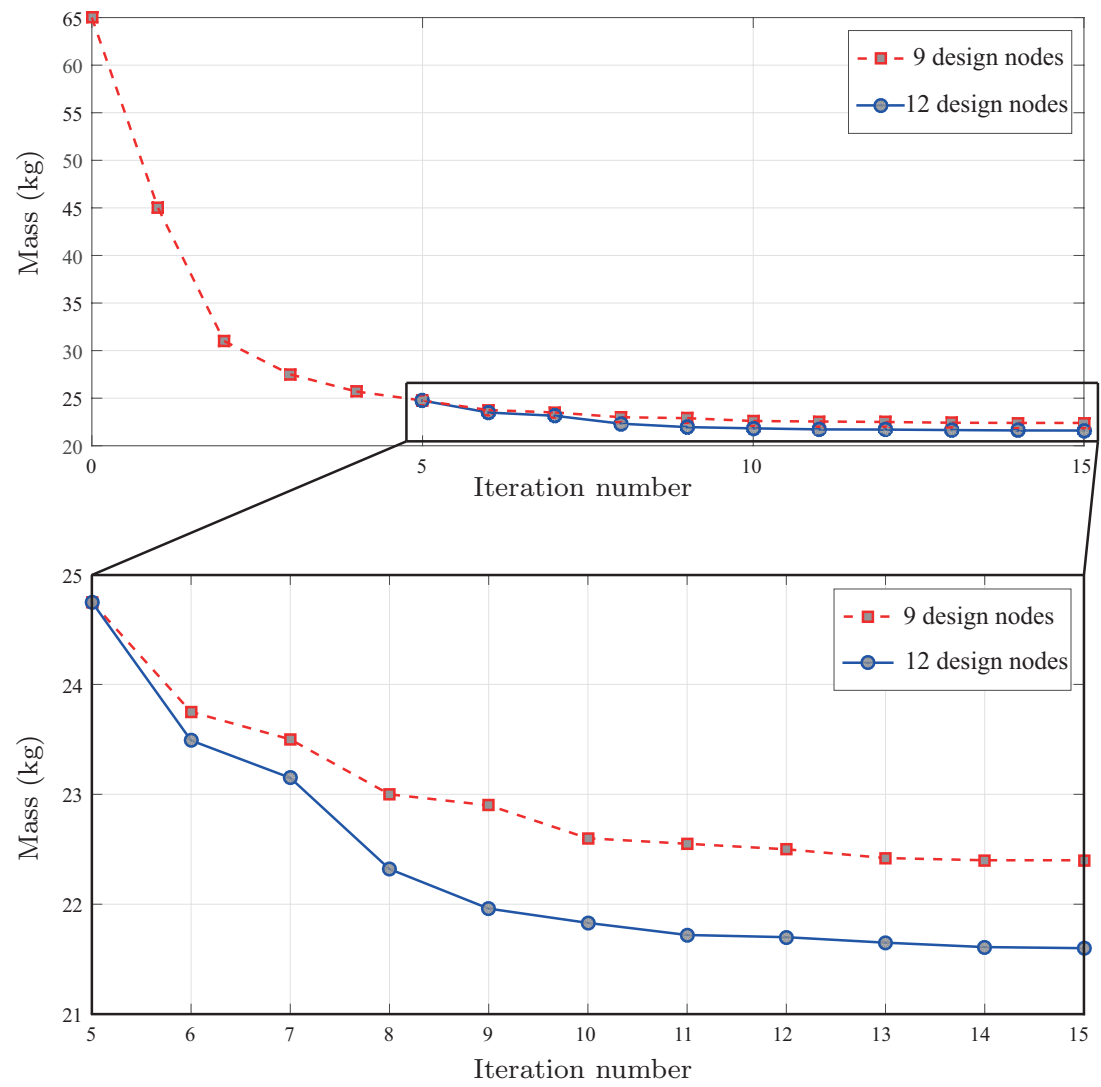

Figure 24: Mass history comparison of the optimization problem with 9 design nodes with that using 12 design nodes. The design of the optimization with 9 design nodes after 5 iterations is used as the initial design of the optimization problem with 12 design nodes. 


\section{Conclusion and outlook}

Optimization of large scale stiffened structures with the goal of mass minimization under local buckling constraint is addressed in the current research. The proposed method separates the optimization of the stacking sequences from the optimization of the thickness distribution. A Stacking Sequence Table (SST) is generated first. A gradient-based optimization is performed to obtain an estimate about the optimal stiffness and thickness distribution over the structure. Using this information optimized stacking sequences that satisfy blending and other required laminate design guidelines were generated. In particular, symmetry, covering ply, disorientation, percentage rule, balance, and contiguity guidelines are addressed in this study.

Next, an auxiliary level-set function is introduced to specify the boundaries of the laminates with different thicknesses from the obtained SST over the structure. The value of the level-set function specifies the boundaries of regions with equal thickness over the structure. As long as the laminates covering the structure are selected from the SST, the blending of the design is guaranteed and the required laminate design guidelines are fulfilled without adding extra constraints to the optimization problem.

Separating the optimization problem is in general cheaper compared to when the stacking sequences and the thickness distribution are optimized simultaneously where blending as well as other laminate design guidelines are added to the structural response (e.g buckling) as constraints of the optimization problem.

The proposed method is efficient as it is straightforward, fast, and compatible with any standard finite element package. The proposed level-set method allows continuous change of the location of the ply drop over the structure using a straightforward approach. As the number of design nodes is independent from the number of finite element nodes, no matter how dense the mesh is, the selection of a very limited number of design nodes makes a large finite element model to be optimized cheaply. Since the finite element model remains unchanged during the optimization process, the generated program (as shown in Figure 6) only updates the input file of a finite element program with element stiffness values; therefore, it can be easily connected to any commercial finite element package.

The proposed level-set method is flexible in terms of the number and the location of design nodes. If in a region of the structure more details are required to be captured, more design nodes can be added locally.

The computation time in the proposed method is directly related to number of design nodes in the level-set-based thickness optimization problem. As described in Section 4, the overall convergence time of a problem can be decreased as part of the optimization procedure can be performed with a smaller number of design nodes.

The choice of the initial design of the level-set-based optimization may result in the final design of the problem to be trapped in a local minimum. As for the initial design, it was suggested to assign values to design variables such that the resulted (area weighted average) thickness distribution is close to the idealized thickness distribution.

In the procedure of generating an SST, a gradient-based optimization algorithm was used. Thanks to the proposed level-set parametrization, the discrete thickness opti- 
mization problem was also solved using a gradient-based algorithm. Solving the entire optimization problem using a gradient-based algorithm is in general faster and less expensive compared to the application of a genetic algorithm.

As an SST determines the stacking sequence of the laminates, the optimum design of the structure is strongly dependent on the generated SST. Using a method more accurate than the smeared stiffness to obtain an idealized design is the subject of future research.

\section{Acknowledgements}

The support of this research by partners in TAPAS2 project is gratefully acknowledged.

\section{Appendix A. SST generation for the Horseshoe Prob- lem}

According to the step 1 procedure shown in Figure 1, the optimization problem defined in Equation (3) was solved using the MATLAB optimization toolbox. As the laminate thickness is constant in each panel and shear loads are excluded, the following equation $[1,17]$ is used to calculate the buckling load in each panel during the procedure of optimization problem in Equation (3).

$$
\lambda(m, n)=\frac{\pi^{2}\left[D_{11}(m / a)^{4}+2\left(D_{12}+2 D_{66}\right)(m / a)^{2}(n / b)^{2}+D_{22}(n / b)^{4}\right]}{(m / a)^{2} N_{x}+(n / b)^{2} N_{y}}
$$

where $m$ and $n$ are the number of half-waves in $\mathrm{x}$ and $\mathrm{y}$ directions, respectively. Here, $m=1,2$ and $n=1,2$ are considered. The dimensions of the panel are $a$ and $b$ in the $x$ and $y$ directions, respectively. $N_{x}$ and $N_{y}$ are the in-plane loads along the $x$ and the $y$ directions, respectively. $D_{11}, D_{12}, D_{22}$, and $D_{66}$ are the components of the bending stiffness matrix.

The sensitivity of the objective function was calculated according to:

$$
\frac{\mathrm{d} f}{\mathrm{~d} n_{r}^{j}}=S_{j} t, \quad j=1 \text { to } N_{\mathrm{p}}, \quad r=0,45,90
$$

where $n_{r}^{j}$ represents the design variable related to the ply with fiber orientation $r$, for the $j^{\text {th }}$ panel. $S_{j}$ and $t$ represent the area of the $j^{\text {th }}$ panel and the ply thickness, respectively. $N_{\mathrm{p}}$ represents the total number of panels.

Considering Equation (2), the sensitivity of a buckling factor to a design variable was analytically calculated as follows:

$$
\begin{aligned}
\frac{\mathrm{d} \lambda(m, n)}{\mathrm{d} n_{r}^{j}} & =\frac{\mathrm{d} \lambda(m, n)}{\mathrm{d} \mathbf{D}}\left(\frac{\mathrm{d} \mathbf{D}}{\mathrm{d} \mathbf{A}} \frac{\mathrm{d} \mathbf{A}}{\mathrm{d} n_{r}^{j}}+\frac{\mathrm{d} \mathbf{D}}{\mathrm{d} h^{j}} \frac{\mathrm{d} h^{j}}{\mathrm{~d} n_{r}^{j}}\right) \\
j & =1 \text { to } N_{\mathrm{p}}, \quad r=0,45,90
\end{aligned}
$$


Table 6: The result of the optimization problem defined in Equation (3). The obtained (rounded) thickness values of the idealized design are compared with those reported as the optimized solution in [1].

\begin{tabular}{|c|c|c|c|c|c|}
\hline Panel & $n_{0}$ & $n_{45}$ & $n_{90}$ & $\begin{array}{l}\text { Conceptual ply number (rounded) } \\
2\left(n_{0}+n_{45}+n_{90}\right)\end{array}$ & $\begin{array}{l}\text { Ply number } \\
\text { Yang [1] }\end{array}$ \\
\hline $\begin{array}{l}1 \\
2 \\
3 \\
4 \\
5 \\
6 \\
7 \\
8 \\
9 \\
10 \\
11 \\
12 \\
13 \\
14 \\
15 \\
16 \\
17 \\
18\end{array}$ & $\begin{array}{l}1.65 \\
1.41 \\
1.04 \\
1 \\
1 \\
1.07 \\
1 \\
1.22 \\
1.91 \\
1.76 \\
1.48 \\
1.41 \\
1.06 \\
1 \\
1.23 \\
1.5 \\
1.11\end{array}$ & $\begin{array}{l}13.21 \\
11.29 \\
2.08 \\
2 \\
2 \\
2.15 \\
2 \\
2.45 \\
15.30 \\
14.10 \\
11.87 \\
11.33 \\
2.12 \\
2 \\
2.47 \\
3.01 \\
2 \\
2.22\end{array}$ & $\begin{array}{l}1.65 \\
1.41 \\
7.27 \\
6.19 \\
4.84 \\
7.53 \\
6.29 \\
8.58 \\
1.91 \\
1.76 \\
1.48 \\
1.41 \\
7.42 \\
6.02 \\
8.67 \\
10.55 \\
6.24 \\
7.78\end{array}$ & $\begin{array}{l}34 \\
28 \\
20 \\
18 \\
16 \\
22 \\
18 \\
24 \\
38 \\
36 \\
30 \\
28 \\
22 \\
18 \\
24 \\
30 \\
18 \\
22\end{array}$ & $\begin{array}{l}34 \\
28 \\
22 \\
20 \\
16 \\
22 \\
20 \\
26 \\
38 \\
36 \\
30 \\
28 \\
22 \\
20 \\
26 \\
32 \\
20 \\
24\end{array}$ \\
\hline
\end{tabular}

where $h^{j}$ represents the thickness of the laminate in the $j^{\text {th }}$ panel: $h^{j}=2 t\left(n_{0}^{j}+n_{45}^{j}+\right.$ $\left.n_{90}^{j}\right)$. As each design variable represents the number of its corresponding plies in a half laminate, the sum of the design variables in each panel is multiplied by 2 .

Table 6 shows the result of the optimization problem defined in Equation (3). To have an indication of the quality of the idealized design, the obtained (rounded to the nearest integer) thickness values are compared with those reported as the optimized solution in [1].

As it can be seen in Table 6 , the thickness value for each panel in the idealized design is in a good agreement with those reported in [1]. According to Table 6, only panels 11 and 16 have the same thickness value, while having considerably different values of design variables. The majority of the plies in panel 11 have $\pm 45^{\circ}$ fiber orientation while panel 16 mainly consists of plies with $90^{\circ}$ fiber orientation. Thus using the idealized design, 2 different SSTs (called SST-A and SST-B) can be generated. Figure 25 shows the two SSTs resulting from the idealized design. Only the stacking sequences of half laminates are shown.

The idealized design gives a constant thickness value per panel. Using the proposed level-set method, however, each panel may include various thickness values. The area weighted average of various thicknesses in each panel is expected to be close to the idealized thickness value obtained for each panel (see Section 4.1). Therefore, as a result of the level-set-based thickness optimization, laminates thicker and thinner than the idealized laminate are also expected in each panel. For this reason, the SSTs shown in Figure 25 include thinner and thicker laminates compared to those obtained in the idealized design (see Table 6) to avoid unnecessarily restricting the design variables [39]. The AD stiffness values of the thickest and the thinnest laminates in 


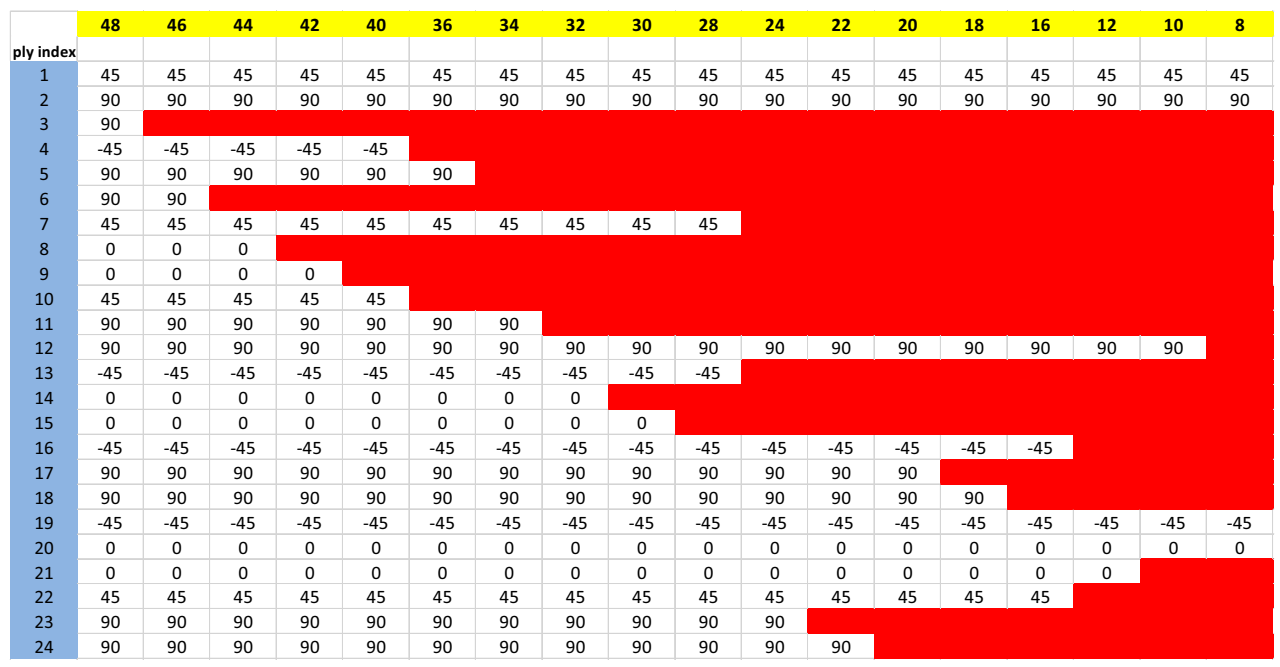

(a) SST-A

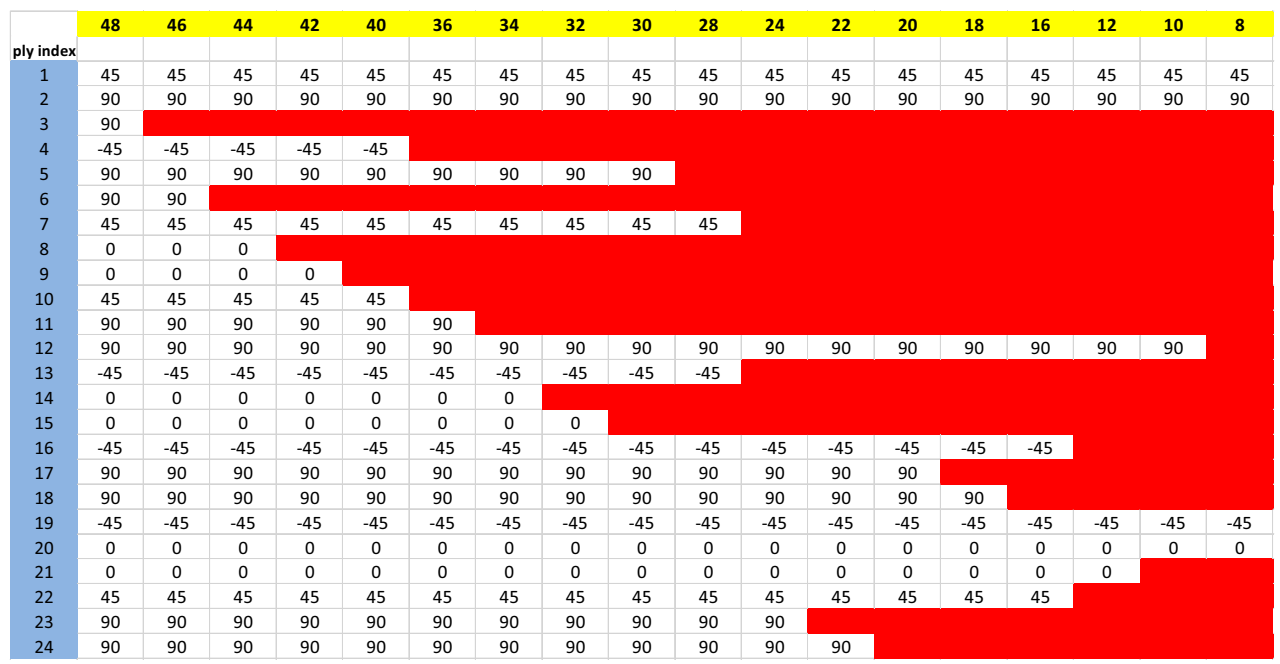

(b) SST-B

Figure 25: Two different SSTs resulting from the idealized design where fields marked red indicate dropped plies. Ply indices (first column from left) are in the ascending order from the outer most ply towards the laminates center. 
Table 7: The result of the buckling analysis for panels 1, 11, and 16 using SST-A and SST-B.

\begin{tabular}{|c|c|c|}
\hline Panel & $\lambda_{\text {cr }}$ using SST-A & $\lambda_{\text {cr }}$ using SST-B \\
\hline $\begin{array}{l}1 \\
11 \\
16\end{array}$ & $\begin{array}{l}0.85 \\
0.84 \\
0.91\end{array}$ & $\begin{array}{l}0.83 \\
0.77 \\
0.99\end{array}$ \\
\hline Min & 0.84 & 0.77 \\
\hline
\end{tabular}

the idealized design were extrapolated to have an estimate of the stiffness values of laminates that are not suggested by the idealized design, but do exist in the SST. The extrapolated stiffness values were used in the selection procedure of the fittest laminate as discussed in Section 2.

For the Horseshoe Problem, Equation (14) can be used to easily calculate the buckling factors. To evaluate the performance of the SSTs shown in Figure 25, Equation (14) is used to directly calculate the buckling factors instead of using the quality indicator defined in Equation (5). Using the idealized thickness distribution (shown in Table 6 ), the structure is subject to buckling analysis. First, stacking sequences are selected from SST-A (see Figure 25), and then the stacking sequences in SST-B are used. As it can be seen in Figure 25, the stacking sequences of laminates with 30, 32, and 34 plies are different between SST-A and SST-B. Therefore, only the stacking sequence of panels 1, 11, and 16 differs as a result of using the two different SSTs. Thus here, only these panels are subjected to buckling analysis (considering the fact that load redistribution is ignored in the Horseshoe Problem). Table 7 shows the result of the buckling analysis for panels 1, 11, and 16 using SST-A and SST-B.

As the minimum critical buckling factor using SST-A is larger than the one obtained using SST-B, SST-A in Figure 25 is selected to be used for thickness optimization. 


\section{References}

[1] J. Yang, B. Song, X. Zhong, and P. Jin. Optimal design of blended composite laminate structures using ply drop sequence. Composite Structures, 135:30 - 37, 2016.

[2] Y. M. Meddaikar, F. X. Irisarri, and M. M. Abdalla. Laminate optimization of blended composite structures using a modified shepard's method and stacking sequence tables. Structural and Multidisciplinary Optimization, 55(2):535-546, 2017.

[3] G. Allaire and G. Delgado. Stacking sequence and shape optimization of laminated composite plates via a level-set method. Journal of the Mechanics and Physics of Solids, 97:168 - 196, 2016.

[4] Military Handbook - MIL-HDBK-17-3F: Composite Materials Handbook, Volume 3 - Polymer Matrix Composites Materials Usage, Design, and Analysis. U.S. Department of Defense, 2002.

[5] J. A. Bailie, R. P. Ley, and A. Pasricha. A summary and review of composite laminate design guidelines. Technical report NASA, NAS1-19347. Northrop Grumman-Military Aircraft Systems Division, 1997.

[6] H. Ghiasi, K. Fayazbakhsh, D. Pasini, and L. Lessard. Optimum stacking sequence design of composite materials part 2: Variable stiffness design. Composite Structures, 93(1):1 - 13, 2010.

[7] D. B. Adams, L. T. Watson, Z. Gürdal, and C. M. Anderson-Cook. Genetic algorithm optimization and blending of composite laminates by locally reducing laminate thickness. Advances in Engineering Software, 35(1):35-43, 2004.

[8] D. B. Adams, L. T. Watson, and Z. Gürdal. Optimization and blending of composite laminates using genetic algorithms with migration. Mechanics of Advanced Materials and Structures, 10:183-203, 2003.

[9] B. P. Kristinsdottir, Z. B. Zabinsky, M. E. Tuttle, and S. Neogi. Optimal design of large composite panels with varying loads. Composite Structures, 51:93-102, 2001.

[10] M. T. McMahon and L. T. Watson. A distributed genetic algorithm with migration for the design of composite laminate structures. Parallel Algorithms and Applications, 14:329-362, 2000.

[11] G. Soremekun, Z. Gürdal, C. Kassapoglou, and D. Toni. Stacking sequence blending of multiple composite laminates using genetic algorithms. Composite Structures, 56(1):53-62, 2002.

[12] B. Liu and R. T. Haftka. Composite wing structural design optimization with continuity constraints. In 19th AIAA Applied Aerodynamics Conference, March 2001. 
[13] D. B. Adams, L. T. Watson, O. Seresta, and Z. Gürdal. Global/local iteration for blended composite laminate panel structure optimization subproblems. Mechanics of Advanced Materials and Structures, 14(2):139-150, 2007.

[14] N. Zehnder and P. Ermanni. A methodology for the global optimization of laminated composite structures. Composite Structures, 72(3):311 - 320, 2006.

[15] N. Zehnder and P. Ermanni. Optimizing the shape and placement of patches of reinforcement fibers. Composite Structures, 77(1):1 - 9, 2007.

[16] J. H. Starnes and R. T. Haftka. Preliminary design of composite wings for buckling, strength, and displacement constraints. Journal of Aircraft, 16(8):564$570,1979$.

[17] F. X. Irisarri, A. Lasseigne, F. H. Leroy, and R. Le Riche. Optimal design of laminated composite structures with ply drops using stacking sequence tables. Composite Structures, 107:559-569, 2014.

[18] M. P. Bendsøe and N. Kikuchi. Generating optimal topologies in structural design using a homogenization method. Computer Methods in Applied Mechanics and Engineering, 71(2):197 - 224, 1988.

[19] M. P. Bendsøe. Optimal shape design as a material distribution problem. Structural optimization, 1(4):193-202, Dec 1989.

[20] G. I. N. Rozvany, M. Zhou, and T. Birker. Generalized shape optimization without homogenization. Structural optimization, 4(3):250-252, Sep 1992.

[21] G. Costa, M. Montemurro, and J. Pailhès. A 2D topology optimisation algorithm in NURBS framework with geometric constraints. International Journal of Mechanics and Materials in Design., 2017.

[22] X. Qian. Topology optimization in B-spline space. Computer Methods in Applied Mechanics and Engineering, 265:15-35, 2013.

[23] M. Stolpe and K. Svanberg. An alternative interpolation scheme for minimum compliance topology optimization. Structural and Multidisciplinary Optimization, 22(2):116-124, Sep 2001.

[24] O. Sigmund and S. Torquato. Design of materials with extreme thermal expansion using a three-phase topology optimization method. Journal of the Mechanics and Physics of Solids, 45(6):1037 - 1067, 1997.

[25] L. V. Gibiansky and O. Sigmund. Multiphase composites with extremal bulk modulus. Journal of the Mechanics and Physics of Solids, 48(3):461 - 498, 2000.

[26] J. Thomsen. Topology optimization of structures composed of one or two materials. Structural optimization, 5(1):108-115, Mar 1992.

[27] C. F. Hvejsel and E. Lund. Material interpolation schemes for unified topology and multi-material optimization. Structural and Multidisciplinary Optimization, 43(6):811-825, Jun 2011. 
[28] S. N. Sørensen and Erik Lund. Topology and thickness optimization of laminated composites including manufacturing constraints. Structural and Multidisciplinary Optimization, 48(2):249-265, Aug 2013.

[29] S. N. Sørensen, R. Sørensen, and E. Lund. DMTO - a method for discrete material and thickness optimization of laminated composite structures. Structural and Multidisciplinary Optimization, 50(1):25-47, Jul 2014.

[30] S. N. Sørensen and M. Stolpe. Global blending optimization of laminated composites with discrete material candidate selection and thickness variation. Structural and Multidisciplinary Optimization, 52(1):137-155, 2015.

[31] E. Lund. Discrete material and thickness optimization of laminated composite structures including failure criteria. Structural and Multidisciplinary Optimization, Dec 2017.

[32] A. Carpentier, L. Michel, S. Grihon, and J. J. Barrau. Buckling optimization of composite panels via lay-up tables. In 3rd European Conference on Computational Mechanics Solids, Structures and Coupled Problems in Engineering, Lisbon, Portugal, June 2006.

[33] F. X. Irisarri, D. M. J. Peeters, and M. M. Abdalla. Optimisation of ply drop order in variable stiffness laminates. Composite Structures, 152:791-799, 2016.

[34] S. T. IJsselmuiden, M. M. Abdalla, O. Seresta, and Z. Gürdal. Multi-step blended stacking sequence design of panel assemblies with buckling constraints. Composites Part B: Engineering, 40(4):329 - 336, 2009.

[35] O. Seresta, M. M. Abdalla, and Z. Gürdal. A genetic algorithm based blending scheme for design of multiple composite laminates. In Collection of Technical Papers - AIAA/ASME/ASCE/AHS/ASC Structures, Structural Dynamics and Materials Conference, 2009.

[36] P. Jin, X. Zhong, J. Yang, and Z. Sun. Blending design of composite panels with lamination parameters. The Aeronautical Journal, 120(1233):1710-1725, 2016.

[37] P. Jin, Y. Wang, X. Zhong, J. Yang, and Z. Sun. Blending design of composite laminated structure with panel permutation sequence. Aeronautical Journal, 122(1248):333-347, 2018.

[38] H. Ghiasi, D. Pasini, and L. Lessard. Optimum stacking sequence design of composite materials part 1: Constant stiffness design. Composite Structures, 90(1):1-11, 2009.

[39] F. Farzan Nasab, H. J. M. Geijselaers, I. Baran, and A. de Boer. Generating the best stacking sequence table for the design of blended composite structures. In Advances in Structural and Multidisciplinary Optimization: Proceedings of the 12th World Congress of Structural and Multidisciplinary Optimization (WCSMO12), pages 779-788, 2018. 
[40] J. Liu and Y. Ma. A new multi-material level set topology optimization method with the length scale control capability. Computer Methods in Applied Mechanics and Engineering, 329:444-463, 2018.

[41] L. A. Vese and T. F. Chan. A multiphase level set framework for image segmentation using the Mumford and Shah model. International Journal of Computer Vision, 50(3):271-293, 2002.

[42] D. Liu, V. V. Toroporov, O. M. Querin, and D. C. Barton. Bilevel optimization of blended composite wing panels. Journal of Aircraft, 48(1):107-118, 2011.

[43] C. Kassapoglou. Design and Analysis of Composite Structures: With Applications to Aerospace Structures. John Wiley and Sons, second edition, 2013.

[44] M. Montemurro, A. Vincenti, and P. Vannucci. A two-level procedure for the global optimum design of composite modular structures-application to the design of an aircraft wing: Part 1: Theoretical formulation. Journal of Optimization Theory and Applications, 155(1):1-23, 2012.

[45] M. Montemurro, A. Vincenti, and P. Vannucci. A two-level procedure for the global optimum design of composite modular structures-application to the design of an aircraft wing: Part 2: Numerical aspects and examples. Journal of Optimization Theory and Applications, 155(1):24-53, 2012.

[46] S. T. IJsselmuiden, M. M. Abdalla, and Z. Gürdal. Optimization of variablestiffness panels for maximum buckling load using lamination parameters. AIAA Journal, 48(1):134-143, 2010.

[47] M. Montemurro and A. Catapano. A new paradigm for the optimum design of variable angle tow laminates. Springer Optimization and Its Applications, 116:375-400, 2016.

[48] M. Montemurro and A. Catapano. On the effective integration of manufacturability constraints within the multi-scale methodology for designing variable angle-tow laminates. Composite Structures, 161:145-159, 2017.

[49] S. W. Tsai and H. T. Hahn. Introduction of composite materials. Technomic Publishing Company, Lancaster, PA, 1980.

[50] V. B. Hammer, M. P. Bendsøe, R. Lipton, and P. Pedersen. Parametrization in laminate design for optimal compliance. International Journal of Solids and Structures, 34(4):415-433, 1997.

[51] J. N. Reddy. Mechanics of laminated composite plates : theory and analysis. Boca Raton : CRC Press, 1997.

[52] J. S. Arora. Introduction to optimum design. Academic Press, Boston, third edition, 2012.

[53] R. T. Haftka and Z. Gürdal. Elements of structural optimization. Springer, 1992.

[54] R. J. Allemang and D. L. Brown. A correlation coefficient for modal vector analysis. In 1st International Modal Analysis Conference, 1982. 



\section{Paper B}

\section{Topology, shape, and thickness optimization of composite structures considering design, manufacturing, and strength related guidelines}

This chapter is based on the paper 'Topology, shape, and thickness optimization of composite structures considering design, manufacturing, and strength related guidelines', submitted to 'Structural and Multidisciplinary Optimization' (2018). 



\title{
Topology, shape, and thickness optimization of composite structures considering design, manufacturing, and strength related guidelines
}

\author{
F. Farzan Nasab ${ }^{1)}$, H.J.M. Geijselaers ${ }^{1)}$, I. Baran ${ }^{1)}$, R. Akkerman ${ }^{1)}$, A. \\ de Boer ${ }^{1)}$
}

Submitted to Structural and Multidisciplinary Optimization

${ }^{1)}$ Applied Mechanics, Engineering Technology, University of Twente, Enschede, The Netherlands

\begin{abstract}
In the present study a method for the simultaneous optimization of the thickness, the shape, and the topology of (thin) laminated composite structures is investigated. A method for the optimization of the stacking sequences and thickness distribution has been recently introduced by the authors. The objective of the present work is to advance the approach to account for shape and topology optimization as well. Design, manufacturing, and strength related guidelines are guaranteed to be satisfied. To have a valid laminate, design guidelines such as symmetry, covering ply, disorientation, percentage rule, balance, and contiguity rule of the layup have to be satisfied. To have a manufacturable design, plies have to be continuous all-over the structure while the laminate thickness and topology varies (design has to be blended). Strength related criteria in relation to failure modes such as buckling are also accounted for. A stacking sequence table (SST) is generated and optimized first. This SST gives valid laminates with optimized fiber orientations with respect to the applied loads. The SST is then used with a level-set function (LSF). The LSF prescribes optimized shape, topology and thickness distributions. Shape and thickness optimization using the proposed method are examined by its successful application to the mass constrained compliance minimization problem studied in literature. Topology and thickness optimization using the proposed method are examined by minimizing the mass of a wing box rib subject to buckling constraint.
\end{abstract}




\section{Introduction}

The high strength to weight ratio of laminated composite structures makes them very attractive specially for aerospace, wind energy, and automotive industry. The design of a composite structure primarily involves the proper determination of the ply stacking sequences and the thickness distribution. Depending on the application, it may also be desirable to design the shape of a structure. Holes may be required in the design of e.g. ribs of aircraft wings to reduce the weight. Therefore, the shape and the topology may be additional variables in the design of a composite structure.

To avoid failure modes such as delamination and transverse matrix crack propagation, some design guidelines are commonly suggested. These guidelines, comprehensively discussed in $[1,2]$, are e.g. symmetry, covering ply, disorientation, percentage rule, balance, and contiguity rule of the layup. When it comes to designing thin laminates, strength related failure modes such as buckling or structural compliance must be considered as well. In addition to the design and the strength related guidelines, manufacturability of the final design has to be guaranteed. To have a manufacturable design, it is crucial that plies are continuous over the structure while the laminate thickness varies. Optimization of a composite structure with all the earlier mentioned variables subject to the design, manufacturing, and strength related guidelines is a very challenging problem $[3-5]$.

The existing literature concerning material and thickness optimization of composite structures considering some of the design and manufacturing guidelines as well as strength related criteria can be divided into two major categories, namely gradient based methods and heuristic methods.

\subsection{Gradient based methods}

The first category consists of studies that provide a fully gradient based approach to solve the optimization problem. Research based on interpolation schemes with penalization can be placed in this category. Interpolation schemes have been widely used in density based topology optimization (see e.g. [6-8]). Density based topology optimization was originally introduced to solve for a two-phase solid-void problem $[6,9]$ with the goal of mass constrained compliance minimization. The interpolation schemes such as SIMP (Solid Isotropic Material with Penalization) [6, 10] and RAMP (Rational Approximation of Material Properties) [7] are used to relax the discrete nature of the solid-void optimization problem. Using SIMP and RAMP, the design variables are allowed to change continuously between 0 and 1 while intermediate densities are penalized. The interpolation schemes were subsequently extended to account for multiple phases in a design domain, see e.g. [11-14]. In Sigmund and Torquato [11] and Gibiansky and Sigmund [12] the SIMP method was extended to design material micro structures.

DMO (Discrete Material Optimization) was introduced by Stegmann and Lund [8] and Lund and Stegmann [15] to obtain a distinct choice of material among a finite set of candidate materials with different stiffnesses, strengths, and densities. The DMO 
allows for the application of gradient based optimization algorithms by relaxation of the discrete material optimization problem. Hvejsel and Lund [14] introduced a new interpolation scheme for DMO by generalizing SIMP and RAMP. The classical DMO method is applicable for optimization of laminated composites with constant thickness [16]. Sørensen and Lund [16] introduced a topology variable to allow for the occurrence of 'no material' in the design domain. Once the optimizer decides to drop a ply in a region in the design domain, 'no material' is assigned to the volume elements of that region. Thus topology variables can change the thickness of laminates. Topology and candidate material variables form a bilinear stiffness parametrization [16]. In [16], the manufacturability is accounted for by enforcing certain predefined large areas, called patches, to be identical.

The DMTO (Discrete Material and Thickness Optimization) approach was introduced in Sørensen et al. [17]. Discrete material and laminate thickness distribution were optimized towards structural mass minimization subjected to constraints on structural criteria such as buckling load factor and eigenfrequencies. However, a downside of the DMTO is that the symmetry guideline cannot be enforced during the optimization process [18].

Penalization schemes that suppress intermediate densities cause the problem to be non-convex and may result in a large number of local minima [7]. The bilinear stiffness parametrization introduced in [16], as mentioned before, also causes the optimization problem to be non-convex even without penalization [19]. Sørensen and Stolpe [19] proposed an alternative parametrization to that used in [16]. Their proposed formulation follows from defining a (mixed) binary non-linear problem instead of introducing penalization to push design variables towards 0 or 1 . This resulted in a convex problem with a guaranteed global optimum.

Stress constrained topology optimization was investigated in e.g. [20-22] for a single material. Multi-material topology optimization problems considering strength related failure criteria such as maximum stress or strain was subsequently studied in Lund [18]. Manufacturing constraints such as contiguity (defined as a limitation on the number of plies with a same orientation being stacked together) and a limitation on the number of dropped plies were taken into account.

Level-set based topology optimization in its original form can be categorized as a fully gradient based method. In level-set based topology optimization a level-set function determines the interface between voids and material. To update a level-set function throughout the optimization procedure, the sensitivity of a response function to the change of the shape of the material domain has to be obtained [23]. Holes in the design can grow and merge using a level-set function. However, topological derivatives are required to nucleate new holes $[23,24]$. The application of the level-set method in the optimization of multiple materials was investigated in $[25,26]$, in which multiple level-set functions were defined and assigned to each material phase. As a result, the number of design variables in the optimization problem increased corresponding to the number of material phases. Level-set based topology optimization of composite structures considering design and strength guidelines was investigated by Allaire and Delgado [5]. The shape and the topology of each ply were introduced as design variable in addition to the fiber orientation and the sequence of the stack. The 
proposed algorithm is, however, unable to account for the global continuity constraint (blending) [5].

\subsection{Heuristic methods}

The second category involves methods that are fully or partially dependent on metaheuristics such as evolutionary algorithms, typically a genetic algorithm (GA). A GA is a search technique well-suited for various optimization problems with discrete design variables [27, 28]. Liu and Haftka [29] proposed a two-level algorithm with blending treated as a constraint to the optimization problem. At the global level optimization, the material composition of each laminate was determined. At the local level, the GA was used to specify the stacking sequence of the plies. The continuity of stacking sequences (manufacturing guideline) among adjacent panels was considered in the local level design. Guide based continuity (blending) introduced by Adams et al. $[30,31]$ is a method that guarantees the continuity of plies all-over a structure. In their method, the thickest laminate was called the guide laminate. Different laminate thicknesses in a design were obtained by dropping plies from the guide laminate. The method, however, restricted the ply drop to be either from the outermost or the innermost ply. Restricting the ply drop to a specific pattern, it ignores a significant part of the design space [3]. The ply drop sequence (PDS) method, introduced by Yang et al. [3], further advanced the guide based method by relaxing the ply drop pattern.

Another approach to obtain globally continuous layers is the patch concept introduced by Zehnder and Ermanni $[32,33]$. Each globally extended layer was called a patch. A patch was optimized by its geometry, material, and material orientation. The patches were finally assembled to give a varying thickness laminate. However, using the patch concept makes it very difficult to solve the optimization problem as the method introduces a large number of variables [34].

Carpentier et al. [35] introduced the idea of the stacking sequence table (SST) to account for design and manufacturing guidelines. An SST is a reference for the stacking sequence of the laminates with different thicknesses. A thinner laminate in an SST is obtained by selectively dropping plies from a thicker laminate. Unlike the guide based approach [30,31], the ply drop does not have to be restricted to a specific pattern. Irisarri et al. [34] and Meddaikar et al.[4] used the SST in combination with a GA. The method proposed in [34] is capable of satisfying all earlier mentioned design and manufacturing guidelines as well as strength related guidelines in the form of buckling constraints.

Although GAs are well suited for optimization problems involving discrete design variables, they are in general more time consuming than gradient-based algorithms [4]. In practice, often finite element analysis is required to evaluate optimization objective and/or constraints. This makes a GA impractical for application to many design problems in practice as a GA requires a large number of function evaluations. 


\subsection{Objective}

An efficient fully gradient-based method has been recently introduced by the authors that separates the optimization of the stacking sequences from the optimization of the thickness distribution [36]. First, an SST was generated based on the idealized stiffness and thickness distribution over the structure. The laminates in an SST satisfy symmetry, covering ply, disorientation, percentage rule, balance, and contiguity of the layup. Then, a level-set function (LSF), in combination with the generated SST, was used to optimize the ply drop locations over the structure.

The objective of the present work is to advance the approach proposed in [36] to account for shape and topology optimization as well. This is achieved by allowing zero thickness laminates in the design. The LSF prescribes the optimized thickness distribution, topology, and shape of the structure such that the constraints of the optimization problem are satisfied. The LSF is defined on the base of design variables that change continuously. Thus the method is well suited for the application of any gradient based optimization algorithm. Only a single LSF is used in the proposed method. This stands in contrast to the level-set based topology optimization methods that apply multiple LSFs to account for multiple material phases [25, 26]. Defining a single LSF to account for the optimization of multiple plies, considerably reduces the number of required design variables.

The validity of the proposed method is examined by its successful application to the mass constrained compliance minimization of a clamped plate. This problem was previously studied by Sørensen and Lund [16] using a fully gradient based algorithm. The proposed method is then applied to the mass minimization problem of a wing box rib subject to buckling constraints while allowing for holes to reduce the weight of the rib.

The rest of this paper is organized as follows: in Section 2, the SST is briefly discussed. In Section 3, optimization of the shape, topology, and thickness distribution is addressed. The results and discussion are provided in Section 4. The work is concluded in Section 5.

Generating an SST is not the main focus of the present work and can be referred to a previous work of the authors (see [36]). However, as optimizing the stacking sequences is a key part in the optimization of composite structures, detailed information on generation of an SST is provided in Appendix A. The procedure of generation of an SST for the second example in Section 4 is given in Appendix B.

\section{$2 \quad$ Stacking sequence table (SST)}

An SST describes the stacking sequence of laminates with different thicknesses. The laminates in an SST have the potential to be used in the design of a structure. To ensure the ply continuity, a thicker laminate can only be obtained by adding plies to a thinner laminate. A ply with the orientation from the set $\left\{0^{\circ},+45^{\circ},-45^{\circ}\right.$, and $\left.90^{\circ}\right\}$ can only be added if the resulting laminate complies with the following design guidelines $[1,2]$ : 


\begin{tabular}{|c|c|c|c|c|c|c|}
\hline $\begin{array}{c}\text { Number } \\
\text { of plies }\end{array}$ & 20 & 18 & 16 & 12 & 10 & 8 \\
\hline \multicolumn{7}{|l|}{ ply index } \\
\hline 1 & 45 & 45 & 45 & 45 & 45 & 45 \\
\hline 2 & 90 & 90 & 90 & 90 & 90 & 90 \\
\hline 3 & -45 & -45 & -45 & & & \\
\hline 4 & 90 & 90 & 90 & 90 & 90 & 90 \\
\hline 5 & -45 & -45 & -45 & -45 & -45 & -45 \\
\hline 6 & 0 & 0 & 0 & 0 & 0 & \\
\hline 7 & 0 & 0 & 0 & 0 & & \\
\hline 8 & 45 & 45 & 45 & & & \\
\hline 9 & 0 & 0 & & & & \\
\hline 10 & 0 & & & & & \\
\hline
\end{tabular}

Figure 1: A generated SST for the second example studied in Section 4. Fields marked 'red' indicate dropped plies. Due to symmetry, only the stacking sequences of half-laminates are shown.

- Symmetry, the laminate should be symmetric with respect to its center line. This guideline is automatically satisfied as only half-laminates are generated.

- Covering plies, the outermost ply has to have the orientation of either $+45^{\circ}$ or $-45^{\circ}$.

- Disorientation, the maximum difference in the orientation of each two adjacent plies is $45^{\circ}$.

- Percentage rule, at least $10 \%$ of the total number of plies in each laminate have to be assigned to each orientation from the set $\left\{0^{\circ},+45^{\circ},-45^{\circ}\right.$, and $\left.90^{\circ}\right\}$.

- Balance, the total number of plies with $+45^{\circ}$ orientation is equal to the total number of plies with $-45^{\circ}$ orientation in a laminate.

- Contiguity, not more than 4 successive plies with a same orientation are allowed to stack together.

It is advantageous to use an SST because then all design and manufacturing guidelines are satisfied without adding extra constraints to the optimization problem. The stacking sequences of the laminates in an SST have to be optimized with respect to the applied loads and the constraints of the optimization problem. Detailed information on generating an SST with optimized stacking sequences can be found in Appendix A. Figure 1 provides an illustration of an SST generated for the second example studied in Section 4. As the laminates are symmetric, only the stacking sequences of half-laminates are shown. The Ply indices (the leftmost column) are in ascending order from the outermost ply towards the laminate's center. 


\section{Thickness, shape, and topology optimization pro- cedure}

An SST provides valid laminates with optimized fiber orientations with respect to the applied loads. However, the optimized ply drop locations on the structure still need to be identified. In this section, an efficient level-set method is introduced for the optimization of the shape, topology and thickness distribution using an SST. The proposed level-set-based method turns the discrete thickness optimization into a continuous problem.

\subsection{The proposed level-set-based method}

A single level-set function is used to determine the boundaries of laminates with different thicknesses in the composite structure. The same level-set function can prescribe shape and topology as well. The level-set function $(\Phi)$ is discretized by interpolation among a limited set of $N_{\phi}$ design nodes with locations $X_{\phi}$. The design nodes are distributed over the structure. The values $\Phi\left(X_{\phi}\right)$ are the design variables in the optimization problem. The value of $\Phi$ at any location $X$ is obtained by the interpolation

$$
\Phi(X)=\sum_{n=1}^{N_{\Phi}} \Gamma^{n}(X) \Phi^{n}
$$

where $\Gamma^{n}(X)$ are interpolation functions and $\Phi^{n}$ represents $\Phi\left(X_{\phi}^{n}\right)$. Here, we used bilinear interpolation functions on quadrilateral domains, spanning multiple finite elements. This indicates that the discretization of $\Phi$ is independent of the finite element discretization. The same $\Phi$ can be used on models of the same structure with different mesh size, e.g. a coarse model for compliance and a fine model for stress evaluation.

In the proposed method, the level-set function is used to select a ply stack from the SST. The number of plies $N_{\text {plies }}$ of the laminate at any point $X$ in the design domain $\Omega$ is determined through the level-set function by:

$$
N_{\text {plies }}(X)= \begin{cases}\max L V_{i} \mid L V_{i} \leq \Phi(X), & \text { if } \Phi(X) \geq \min L V_{i}, L V_{i} \in \mathrm{LV} \\ 0, & \text { otherwise }\end{cases}
$$

where LV represents the set of all ply stack values in the SST. According to Equation (2) the highest ply stack value below the level-set function at point $X$ gives the number of plies of the laminate covering point $X$. Also, if the level-set function at any point in the design domain $\Omega$ falls below the thinnest ply stack of the SST, no material will be assigned to that point and a void occurs in the design. Figure 2 shows a level-set function covering a structure and the resulting thickness distribution. As it can be seen in Figure 2, part of the level-set function which is below the first level (8 plies) prescribes a void in the design. The ply stacks are selected from the SST e.g. Figure 1. The LV set for the SST shown in Figure 1 is: $\{8,10,12,16, \ldots\}$ plies. 


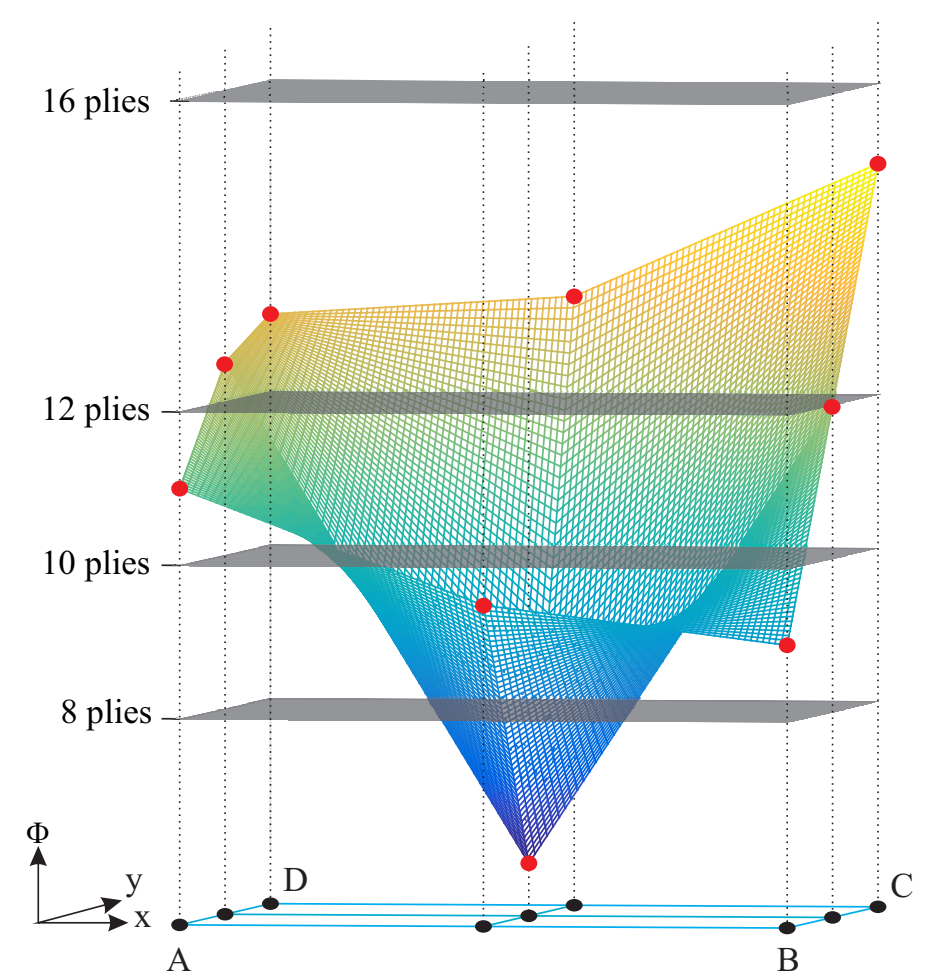

(a) The surface shows a level-set function covering a structure. The red dots indicate the design variables and the black dots indicate the design nodes.

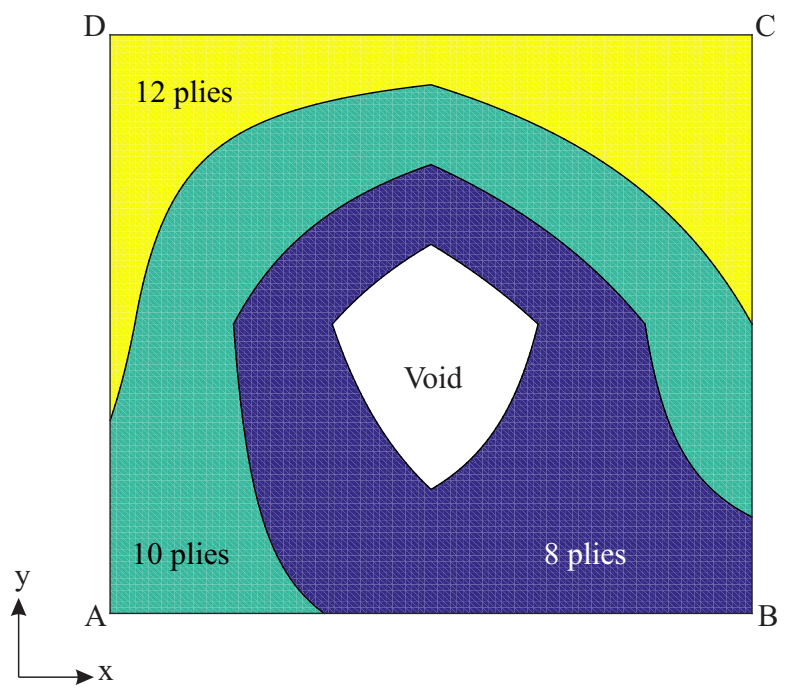

(b) Thickness distribution and the void specified by the levelset function.

Figure 2: Determining thickness distribution and voids using a level-set function. The levels are the ply stack values in the SST shown in Figure 1. 


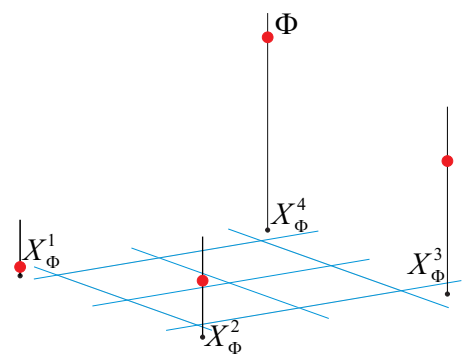

(a) Step 1: only the design nodes (at $X_{\phi}^{i}$ ) have level-set function values. These values are indicated by 'red' dots.

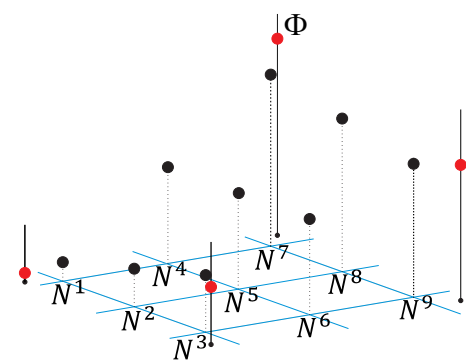

(b) Step 2: each finite element node $N^{i}$ obtains a level-set function value through the design node values interpolation.

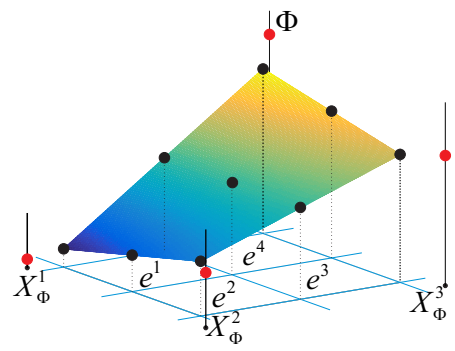

(c) Step 3: the level-set function within the element $e^{i}$ is defined by interpolating the element nodal values.

Figure 3: The interpolation procedure to define a level-set function using the design node values is shown in 3 steps.

The number and the location of the design nodes can be specified depending on the required details for the design. A larger number of design nodes can provide a more detailed design. The number of design nodes directly affects the computation time at each iteration and the number of iterations of the optimization problem. Therefore, a reasonable number of design nodes is suggested to obtain a desirable objective value.

Each finite element node will be assigned a level-set function value based on interpolation from the design node values. Within an element the interpolation of the value of the element nodes specifies the level-set function. Figure 3 schematically shows this procedure in three steps. Each finite element obtains area weighted stiffness data based on the area that each SST laminate covers in the element.

Using the proposed parametrization, the continuous change of a design variable changes the thickness distribution and the topology in the design. Therefore, a change in a design variable changes the elements stiffness matrices. This is the basis of the sensitivity analysis to iteratively update the design using any gradient based algorithm. Here, the 'constrained steepest-descent' algorithm [37] is used to solve the optimization problem. Figure 4 schematically shows the change of the thickness dis- 


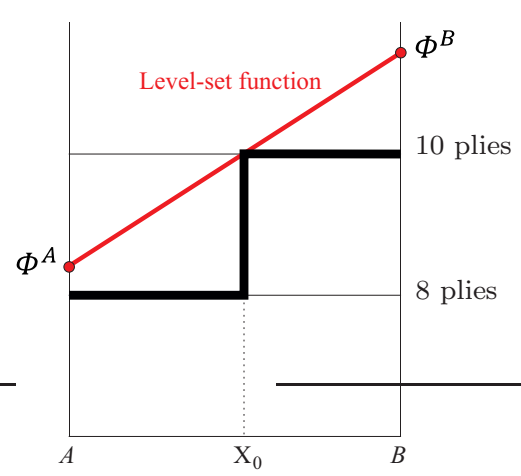

(a) The pq line represents a level-set function. The resulting thickness distribution is shown with the black solid line. $\mathrm{X}_{0}$ indicates the ply drop location.

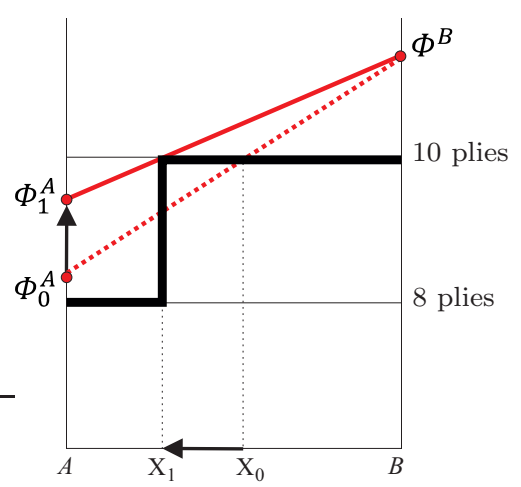

(b) As a result of the continuous change of the design variable $\mathrm{p}_{0}$ by $\mathrm{dp}$, the ply drop location changes continuously from $\mathrm{X}_{0}$ towards $\mathrm{X}_{1}$.

Figure 4: Continuous change of the thickness distribution as a result of continuous change of design variables.

tribution as a result of the continuous change of design variables. The flowchart of the optimization procedure using the level-set method is shown in Figure 5.

\subsection{Optimization objective, constraint, and sensitivity analy- sis}

Here, two common types of engineering problems in aircraft structural optimization are discussed and the obtained results are presented in Section 4. First, mass constrained compliance minimization is discussed. Then, mass minimization subject to buckling constraints is addressed.

\section{Compliance minimization problem}

The compliance minimization problem subject to a mass constraint is formulated as follows:

$$
\begin{aligned}
\min & C\left(\Phi^{N}\right)=f^{T} \mathbf{K}\left(\Phi^{N}\right)^{-1} f=f^{T} \mathbf{u}\left(\Phi^{N}\right) \\
\text { subject to } & M\left(\Phi^{N}\right) \leq M_{\mathrm{a}} \\
& \Phi_{\min } \leq \Phi^{n} \leq \Phi_{\max }
\end{aligned}
$$

where $C, \mathbf{K}, \mathbf{u}$, and $M$ represent, respectively, the system compliance, the system stiffness matrix, the vector of degrees of freedom of the system, and the structural mass as functions of the design variables vector $\Phi^{N}$. The load vector is represented by $f$ and $M_{\mathrm{a}}$ is the allowable mass. $\Phi_{\min }$ and $\Phi_{\max }$ denote lower bound and upper bound for the values at the design nodes, respectively. A finite element simulation is performed to calculate the system compliance at each design. 


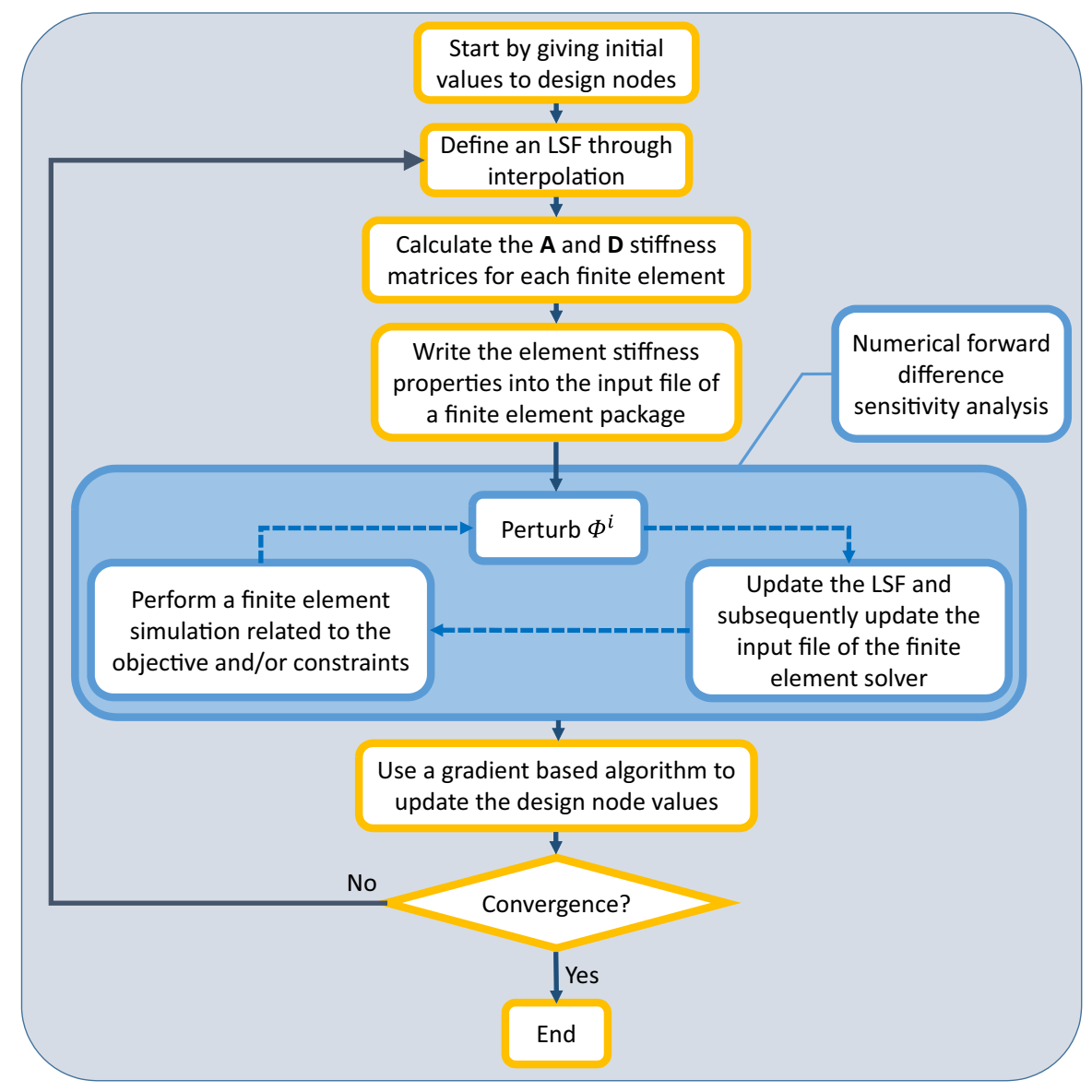

Figure 5: The level-set-based optimization framework. 
The numerical forward difference scheme is used to calculate the compliance sensitivity with respect to a change in the $i^{\text {th }}$ design variable:

$$
\frac{\partial C}{\partial \Phi^{i}}=\frac{C\left(\Phi^{N}+\delta \Phi^{i} \cdot \Phi_{0}^{i}\right)-C\left(\Phi^{N}\right)}{\delta \Phi^{i}}
$$

where $\Phi_{0}^{i}$ is a vector with the same size as $\Phi^{N}$ with the $i^{\text {th }}$ component (corresponding to the $i^{\text {th }}$ design variable) equal to 1 and the rest equal to 0 .

The sensitivity of the mass constraint in Equation (3) can be translated into the sensitivity of the structural mass to a change in the $i^{\text {th }}$ design variable and is calculated in the same fashion as the compliance sensitivity.

\section{Mass minimization problem}

The mass minimization problem subject to buckling constraint can be formulated as follows:

$$
\begin{aligned}
\min & M\left(\Phi^{N}\right) \\
\text { subject to } & g_{i}\left(\Phi^{N}\right) \leq 0 \quad i=1 \text { to } N_{\mathrm{c}} \\
& \Phi_{\min } \leq \Phi^{n} \leq \Phi_{\max }
\end{aligned}
$$

where $M$ represents the overall mass of the structure as a function of design variables vector $\Phi^{N}$. The $i^{\text {th }}$ (buckling) constraint of the problem is represented by $g_{i}$ and $N_{\mathrm{c}}$ is the total number of constraints. The structural mass is calculated as follows:

$$
M=\rho \sum_{i=1}^{e} \sum_{j=1}^{L V} A_{i j} \cdot N_{i j} \cdot t
$$

where $\rho$ is the ply density, $e$ is the total number of elements, $L V$ is the total number of levels in an element, $A_{i j}$ is the area that level $j$ covers in element $i, N_{i j}$ is the number of plies corresponding to the level $j$ in element $i$, and $t$ is the ply thickness.

As the thickness distribution is obtained through the LSF value, a good approximation of the structural mass can be obtained by the integration of the LSF over the structural domain $\Omega$ :

$$
M \approx \rho \int \Phi(X) \mathrm{d} \Omega
$$

Substituting $\Phi(X)$ from Equation (1) into Equation (7), one can write:

$$
M \approx \rho \int \sum_{n=1}^{N_{\Phi}} \Gamma^{n}(X) \Phi^{n} \mathrm{~d} \Omega=\rho \sum_{n=1}^{N_{\Phi}}\left(\int \Gamma^{n}(X) \mathrm{d} \Omega\right) \Phi^{n}=\rho \sum_{n=1}^{N_{\phi}} S^{n} \Phi^{n}
$$

where $S^{n}$ represents the integral of the shape function of design node $n$. According to Equation (8) the optimization objective is a linear function of design variables. Therefore, the sensitivity of the objective function with respect to the $i^{\text {th }}$ design variable can be simply obtained through:

$$
\frac{\partial M}{\partial \Phi^{i}}=\rho S^{i}
$$


The buckling constraint in Equation 5 can be written as:

$$
g_{i}: 1-\lambda_{i} \leq 0 \quad i=1 \text { to } N_{\mathrm{c}}
$$

where $\lambda_{i}$ is the $i^{\text {th }}$ load multiplier or the buckling factor. Solving the following eigenvalue equation gives $\lambda_{i}$ :

$$
\left(\mathbf{K}_{\mathrm{B}}-\lambda_{i} \mathbf{K}_{\mathrm{G}}\right) \cdot w_{i}=0
$$

where $\mathbf{K}_{\mathrm{B}}$ is the global bending stiffness matrix and $\mathbf{K}_{\mathrm{G}}$ is the global stress stiffness matrix. The solution of Equation (11) gives a number of eigenvalues $\lambda$ equal to the rank of $\mathbf{K}_{\mathrm{G}}$. The vector $w_{i}$ is the mode shape corresponding to the $i^{\text {th }}$ buckling factor.

The constraint sensitivity in Equation (5) can be translated into the sensitivity of a buckling factor $\lambda_{i}$ to a change in the $j^{\text {th }}$ design variable and can be obtained through:

$$
\frac{\partial \lambda_{i}}{\partial \Phi^{j}}=\frac{\lambda_{i}\left(\Phi^{N}+\delta \Phi^{i} \cdot \Phi_{0}^{i}\right)-\lambda_{i}\left(\Phi^{N}\right)}{\delta \Phi^{j}}
$$

In Equation (12) it is crucial that the two eigenvalues (eigenvalues before and after perturbation) being subtracted from each other belong to the same mode shape. To ensure this, modal assurance has to be performed. Detailed information on modal assurance analysis can be found in [38].

\section{Results and discussion}

To examine the validity and the performance of the proposed method it is applied to the shape, topology, and thickness optimization of two problems. The cantilever plate problem studied by Sørensen and Lund [16] is examined in the first example. Shape and thickness optimization using the proposed method are investigated in this example.

In the second example, a wing box rib is the target structure for optimization. A rib typically includes lightening holes. Thus the capability of the proposed method to obtain topology as well as thickness distribution is examined in the second example. In both examples, the optimization convergence criterion is defined as $\left\|\mathbf{d}^{(k)}\right\| \leq \epsilon_{1}$ and the maximum constraint violation $V_{k} \leq \epsilon_{2}$, where $\left\|\mathbf{d}^{(k)}\right\|$ denotes the norm of the search direction at iteration $k$ and $\epsilon_{1}$ and $\epsilon_{2}$ are two small positive numbers [37].

\subsection{Example 1, clamped plate}

Mass constrained compliance minimization of a clamped plate, shown in Figure 6, is studied in this example. Following [16], the plate is discretized with 1452 square shell elements with side length $l=0.01 \mathrm{~m}$. ABAQUS 6.12 finite element package is used to calculate the compliance of this structure. Each ply is made of Glass Fiber Reinforced Polymer (GFRP) material where $E_{1}=34 \mathrm{GPa}, E_{2}=E_{3}=8.2 \mathrm{GPa}, G_{12}=G_{13}=4.5$ $\mathrm{GPa}, G_{23}=4.0 \mathrm{GPa}, \nu_{12}=0.29$, and $\rho=1910.0 \mathrm{~kg} / \mathrm{m}^{3}$. The material properties 


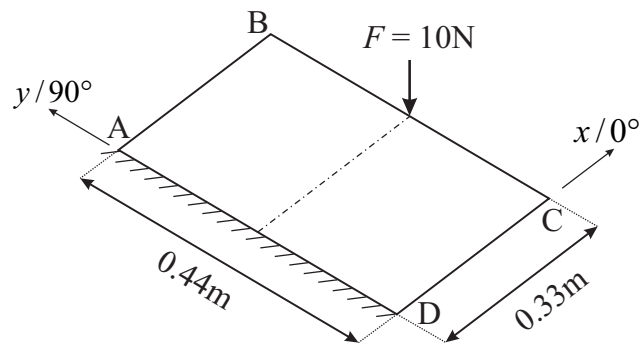

Figure 6: The clamped plate configuration studied in Example 1. The plate is subject to the load $F=10 \mathrm{~N}$.

Table 1: The optimized fiber orientations of Example 1 reported in [16]. Layer 1 represents the innermost ply.

\begin{tabular}{cccccccc}
\hline Layer & 1 & 2 & 3 & 4 & 5 & 6 & 7 \\
\hline Fiber orientation & 0 & 0 & 0 & 90 & 0 & 0 & 0 \\
\hline
\end{tabular}

representing void, are specified as an isotropic material with $E=10^{-6} E_{1}$ and the same Poisson's ratio, $\nu_{12}=0.29$. As in [16], the ply thickness is set to $t=0.001 \mathrm{~m}$ and the allowable mass is $M_{\mathrm{a}}=1 / 2 M_{\max }$ where $M_{\max }$ is the mass corresponding to a domain with dimensions $0.33 \times 0.44 \times 0.007 \mathrm{~m}$.

Two different approaches are examined to solve this problem:

\section{Approach 1}

The purpose of Approach 1 is to obtain an optimized thickness distribution that results in a minimized compliance. The resulting compliance value will be compared with that reported in Sørensen and Lund [16]. They reported the optimized fiber orientations for each layer as shown in Table 1. The SST used in this approach is generated based on the optimized fiber orientations found in [16] (see Table 1). This SST is shown in Figure 7. As seen in Figure 7, the thickest laminate to be used for the proposed level-set method has 9 layers while the thickest laminate reported in [16] has 7 layers. The reason why thicker laminates are included in the SST, will be discussed later in this section.

The optimization problem is formulated as described in Equation (3). $\Phi_{\min }$ is set to be 1 ply and $\Phi_{\max }$ is set to be 9 plies.

The geometry of the structure in Figure 6 is symmetric with respect to the load. Due to the limited set of $\left\{0^{\circ}, 90^{\circ}\right\}$ of fiber orientations, the material properties are also symmetric with respect to the structural symmetry line. Therefore, the concentrated load causes a symmetric deformation in the plate. The regions close to the center of the clamped support (edge AD in Figure 6) are subject to a relatively large bending moment. Thus thicker laminates are expected in those regions. However, around the 


\begin{tabular}{|c|c|c|c|c|c|c|c|c|c|}
\hline $\begin{array}{l}\text { Number } \\
\text { of plies }\end{array}$ & 9 & 8 & 7 & 6 & 5 & 4 & 3 & 2 & 1 \\
\hline \multicolumn{10}{|l|}{ layer } \\
\hline 9 & 0 & & & & & & & & \\
\hline 8 & 0 & 0 & & & & & & & \\
\hline 7 & 0 & 0 & 0 & & & & & & \\
\hline 6 & 0 & 0 & 0 & 0 & & & & & \\
\hline 5 & 0 & 0 & 0 & 0 & 0 & & & & \\
\hline 4 & 90 & 90 & 90 & 90 & 90 & 90 & & & \\
\hline 3 & 0 & 0 & 0 & 0 & 0 & 0 & 0 & & \\
\hline 2 & 0 & 0 & 0 & 0 & 0 & 0 & 0 & 0 & \\
\hline 1 & 0 & 0 & 0 & 0 & 0 & 0 & 0 & 0 & 0 \\
\hline
\end{tabular}

Figure 7: The SST used in Approaches 1 and 2 of Example 1. Fields marked 'red' indicate dropped plies.

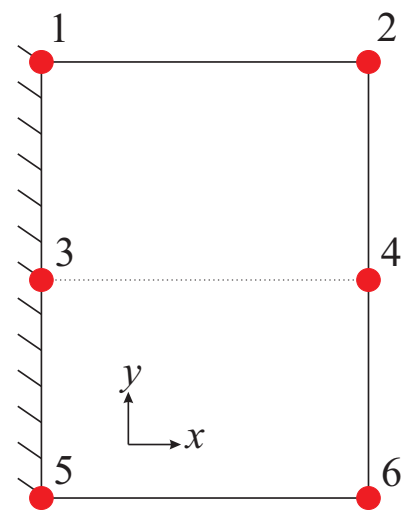

Figure 8: The distribution and the numbering of 6 design nodes.

corners B and C, no significant bending stiffness is required. Thus these regions are expected to be covered with laminates with least thicknesses.

Six design nodes are used to optimize this problem. Figure 8 shows the locations of the design nodes.

Figure 9 shows the contour plot of the thickness distribution at the point of optimum. The optimization problem converges in 4 iterations where each iteration takes about 4 minutes on a regular PC (CPU: $2.6 \mathrm{GHz}, \mathrm{RAM}: 8 \mathrm{~GB}$ ). The optimized compliance value for the problem with 6 design nodes is $C=6.866 \mathrm{e}-3$. This value is larger than that $(C=5.715 \mathrm{e}-3)$ reported in [16]. An explanation is that the assigned number of design nodes is not enough to accurately prescribe a design. To give more freedom to the optimization problem to prescribe thicker laminates on the support edge, two more design nodes are assigned to the edge $\mathrm{AD}$ (see Figure 6). Also, two more design nodes are assigned to the edge BC so that thinner laminates can be assigned locally 


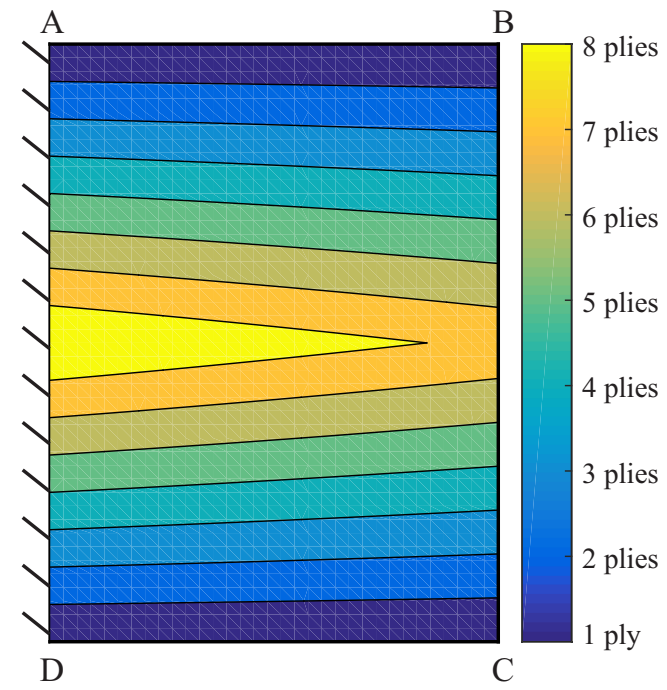

Figure 9: Contour plot of the thickness distribution for the optimization problem in Approach 1 with 6 design nodes.

and more accurately. The new configuration of the design nodes distribution is shown in Figure 10. The optimum design of the problem with 6 design nodes is used for the initial design of the optimization problem with 10 design nodes. This represents an interesting aspect of the proposed optimization method: an optimization problem can start with a small number of design nodes. After some iterations when the design is close to optimum, more design nodes can be added locally to capture a more detailed design. Compared to the optimization problem starting with a larger number of design nodes, less computation is required since part of the optimization problem proceeds with smaller number of design nodes. Figure 11 shows the contour plot of the thickness distribution at the optimized point of the problem with 10 design nodes. As it can be seen in Figure 11, a more detailed contour lines appear close to the regions where the new design nodes are added. Figure 12 shows the compliance evolution for the optimization of both 6 and 10 design nodes.

Table 2 shows the compliance values obtained using 6 design nodes, 10 design nodes, and that reported in Sørensen and Lund [16]. As it can be seen in the table, the compliance value obtained with 10 design nodes is noticeably smaller than that obtained with 6 design nodes. Also, the optimized design using 10 design nodes is less compliant than that obtained in [16]. As the stacking sequence of the laminates is identical to that reported in [16], the resulting compliance is achieved only thanks to the proper thickness distribution prescribed by the level-set function.

There is a significant difference between the proposed parametrization and the parametrization used in the density based topology optimization. In the latter, the design domain is fixed. The design domain is discretized with 7 layers in [16]. Therefore, thicker laminates cannot be prescribed even if they are required. If the design domain were 


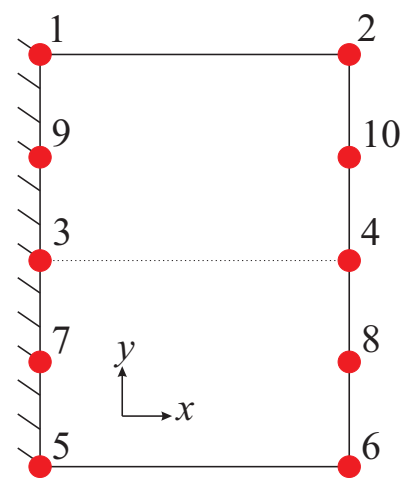

Figure 10: The distribution and the numbering of 10 design nodes.

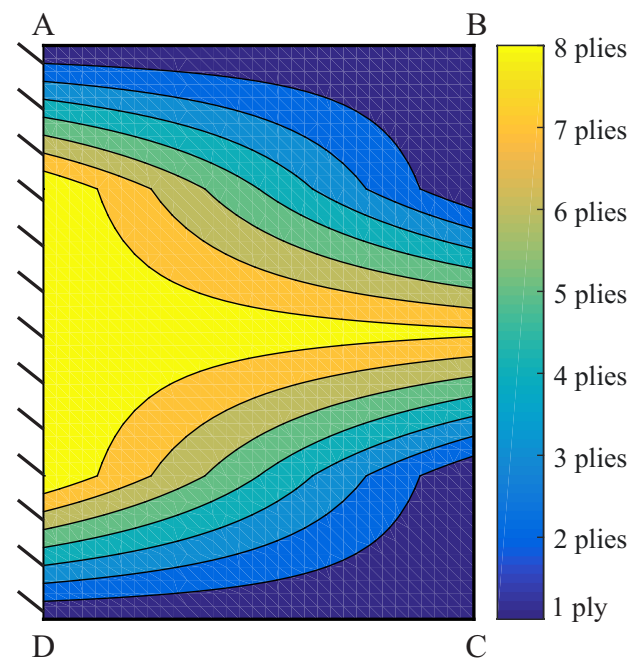

Figure 11: Contour plot of the thickness distribution for the optimization problem in Approach 1 with 10 design nodes. 


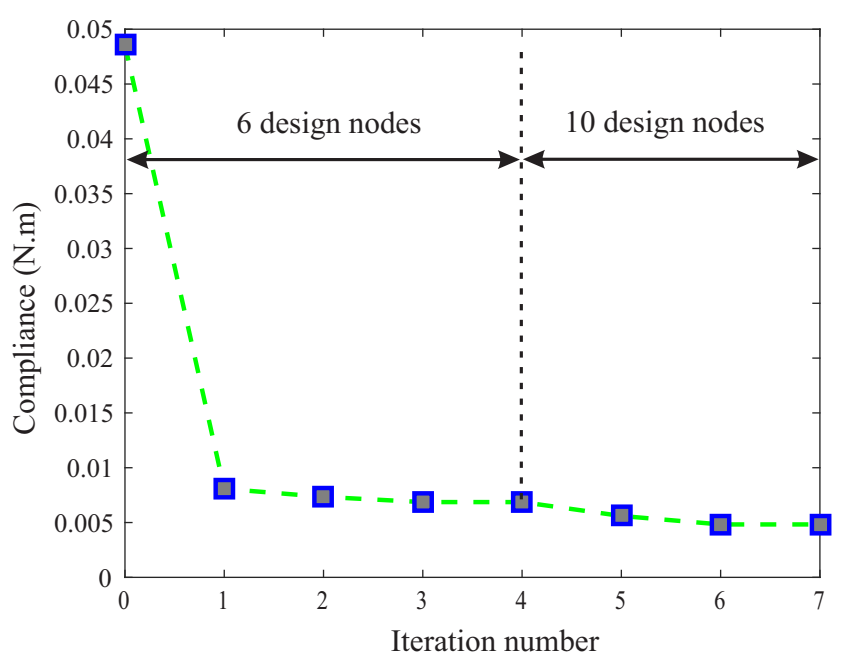

Figure 12: Compliance evolution for the optimization of 10 design nodes in Approach 1. The optimized design of the problem with 6 design nodes (iteration 4 ) is used as the initial design for the optimization problem with 10 design nodes.

Table 2: The compliance value obtained in Approach 1 and that reported in[16].

\begin{tabular}{ccc} 
Solution method & Compliance (N.m) & Method \\
\hline 6 design nodes & $6.866 \mathrm{e}-3$ & Proposed level-set method \\
10 design nodes & $4.827 \mathrm{e}-3$ & Proposed level-set method \\
Sørensen-Lund $[16]$ & $5.715 \mathrm{e}-3$ & Density based topology optimization \\
\hline
\end{tabular}

discretized into a larger number of layers, the number of design variables would have increased which would result in a more expensive and complex optimization problem. In the proposed method, however, an SST can be generated without restricting the laminate thickness values (see Appendix A). Design variables can continuously change in the range of thinnest and the thickest laminates of the SST. As it can be seen in Figure 11, a laminate with 8 layers appears in the design. Allowing design variables to prescribe thicker laminates results in a more optimized mass distribution. This is an explanation for the less compliant design obtained with the same allowable mass using the proposed method.

\section{Approach 2}

In Approach 2, the capability of the proposed method in shape optimization as well as thickness optimization is investigated. Shape optimization using the level-set-based method, i.e. presence of empty space or void, is achieved by allowing design variables on the boundary of the structure to obtain values smaller than the value of the first level in the SST. Shape optimization provides more freedom to remove mass from 


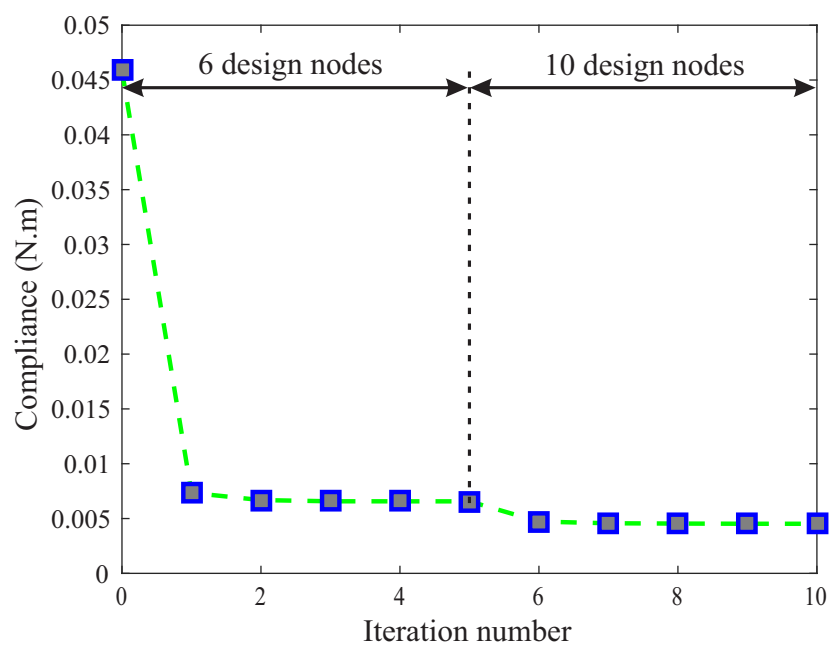

Figure 13: Compliance evolution for the optimization of 10 design nodes in Approach 2. The optimized design of the problem with 6 design nodes (iteration 5 ) is used as the initial design for the optimization problem with 10 design nodes.

the regions where it is not required and assign it to other critical regions. Therefore, it is expected that an even less compliant design will be obtained compared to that presented in Approach 1.

The SST used in Approach 1 is also used in Approach 2. The optimization starts with 6 design nodes with the configuration shown in Figure 8. Design variables are allowed to obtain values smaller than the value of the first level in the SST. The optimization problem converges in 5 iterations. Then, the obtained level-set function is interpolated to 10 design nodes with the configuration shown in Figure 10. Figure 13 shows the progress of the compliance towards the optimum point with both 6 and 10 design nodes. The contour plot of the optimized shape and thickness distribution is shown in Figure 14. As it is seen, as a result of optimization, no material is assigned to the regions around the corners $\mathrm{B}$ and $\mathrm{C}$ of the plate in Figure 6 . The thickest laminate in the optimized design is found to have 8 plies. This thickness also appeared in the design of Approach 1 (see Figure 11). However, the laminate with 8 plies has a larger spread over the structure compared to that of the laminate with the same thickness in Approach 1. This is caused by a more optimized mass distribution when shape optimization is allowed. A design with shape optimization (as well as thickness optimization) results in a less compliant design compared to the case where only the thickness distribution was optimized. Table 3 summarizes the final compliances obtained in Approaches 1 and 2. 


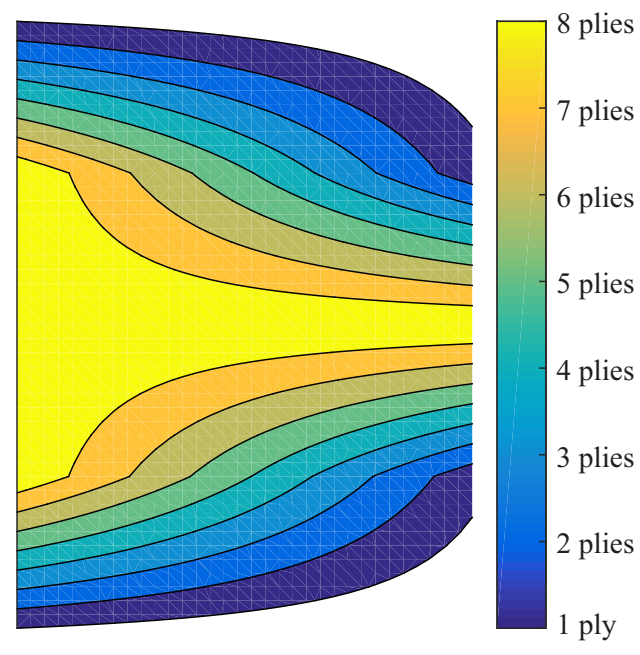

Figure 14: Contour plot of the optimized shape and thickness distribution for the optimization problem in Approach 2.

Table 3: The final compliance values obtained in Approaches 1 and 2.

\begin{tabular}{cc} 
& Compliance (N.m) \\
\hline Approach 1 & $4.827 \mathrm{e}-3$ \\
Approach 2 & $4.524 \mathrm{e}-3$ \\
\hline
\end{tabular}




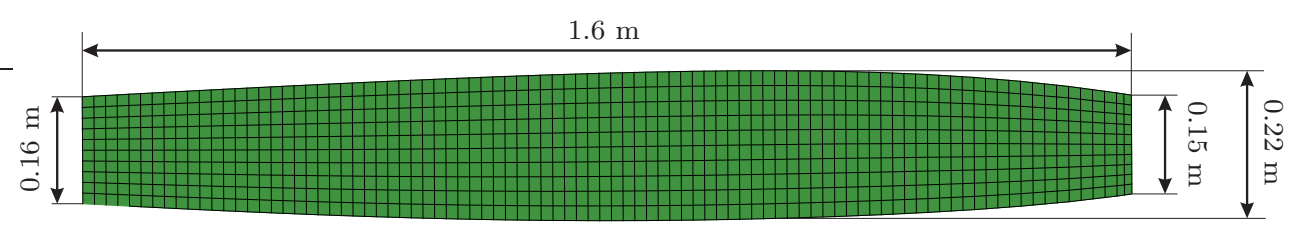

Figure 15: The geometry and the finite element discretization of the rib in Example 2 .

\subsection{Example 2, rib optimization}

Mass minimization of a wing box rib is studied in Example 2. This rib is subject to a compressive crushing load as a result of the torsion box deflection [39, 40]. The crushing load may cause buckling of the rib. Therefore, buckling is considered as the constraint of the optimization problem. As a rib may contain lightening holes, the capability of the proposed method in prescribing topology with optimized thickness distribution is investigated in this example.

The rib geometry is shown in Figure 15. The entire rib is discretized into 900 four node shell elements. The ABAQUS 6.12 finite element package is used for buckling analyses. In addition to the earlier mentioned crushing loads, shear loads are also applied to the rib from the top and the bottom skins of a wing box. The distribution of the compressive and the shear load applied to the rib is shown in Figure 16. The rib is simply supported and the out of plane translational degree of freedom of all boundary nodes is suppressed.

The SST shown in Figure 1 is used as a reference for the stacking sequences in this example. According to the SST the LV set is $\{8,10,12,16,18,20\}$ plies. This SST is optimized for an improved buckling performance under the applied loads shown in Figure 16 (see Appendix B).

The optimization problem is formulated as described in Equation (5). $\Phi_{\min }$ is set to be 8 plies for design nodes on the boundary of the structure. The rest of the design nodes are allowed to obtain values smaller than the value of the first level. $\Phi_{\max }$ is set to be 20 plies. 10 mode shapes are considered as buckling constraints of the problem.

For the construction of each ply in this example Carbon Fiber Reinforced Polymer $(\mathrm{CFRP})$ with the following mechanical properties is used: $E_{1}=126.1 \mathrm{GPa}, E_{2}=11.2$ $\mathrm{GPa}, G_{12}=5.46 \mathrm{GPa}$, and $\nu_{12}=0.3$. The ply thickness is set to $0.13 \mathrm{e}-3 \mathrm{~m}$. The stiffness properties of the void region is set to be $10^{-6}$ times the aforementioned properties where the Poisson's ratio remains the same.

Figure 17 shows the first four buckling mode shapes of the rib when it is covered with a constant thickness laminate (here, 20 plies). It is expected that the regions where buckling is dominant will be covered with relatively thicker laminates.

The rib is initially aimed to be optimized with 12 design nodes as shown in Figure 18. A laminate with a constant thickness equal to 18 plies is used for the initial design. 


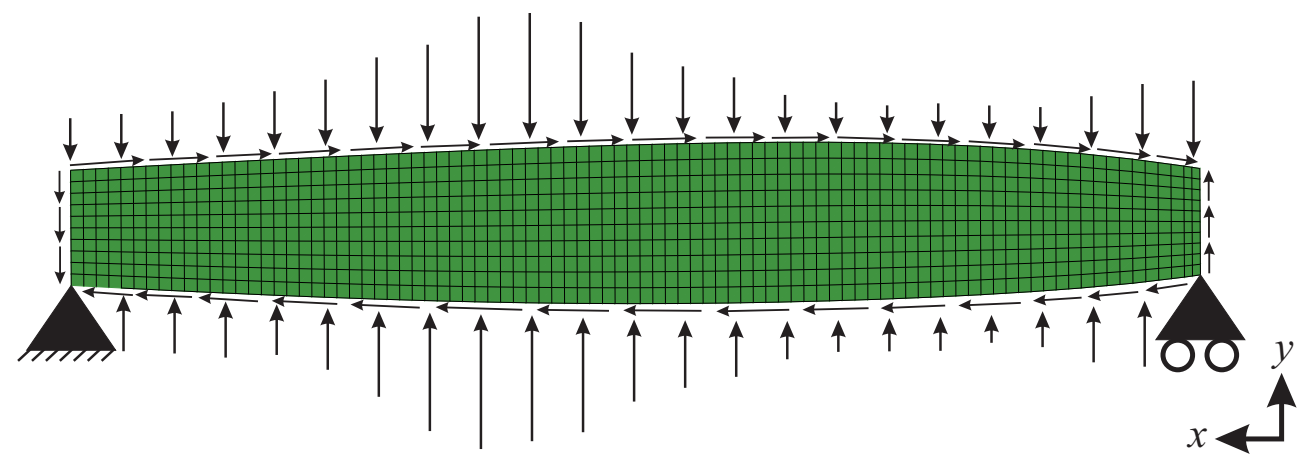

Figure 16: The compressive and the shear loads applied to the rib. The length of the arrows corresponds to the relative magnitude of the load. The rib is simply supported as can be seen. The out of plane translational degree of freedom of all boundary nodes are suppressed.

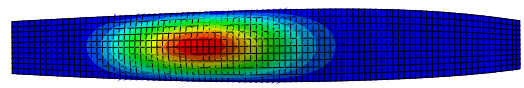

(a) Mode 1

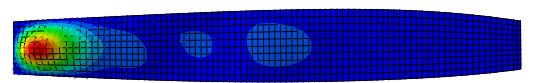

(c) Mode 3

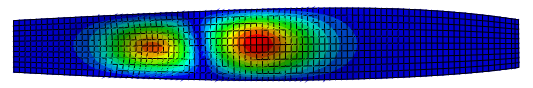

(b) Mode 2

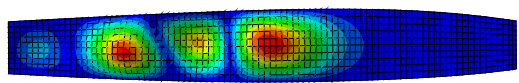

(d) Mode 4

Figure 17: The first four buckling mode shapes where the rib is covered with a constant thickness laminate of 20 plies. The stacking sequence of this laminate is selected from the SST shown in Figure 1. The rib is subjected to loads with a configuration shown in Figure 16. 


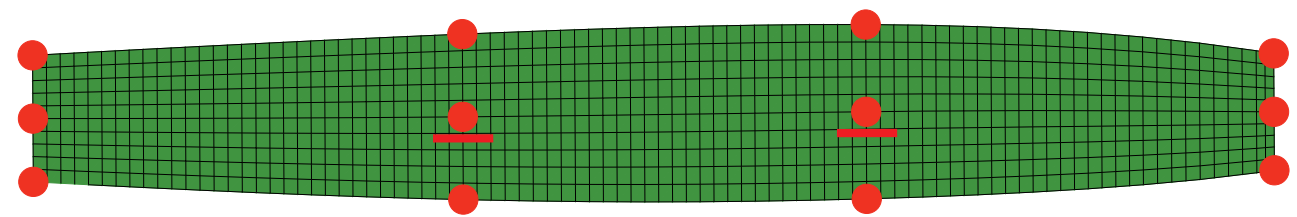

Figure 18: The distribution of the 12 design nodes. The design nodes that are allowed to obtain values smaller than the value of the first level are indicated with an underline.

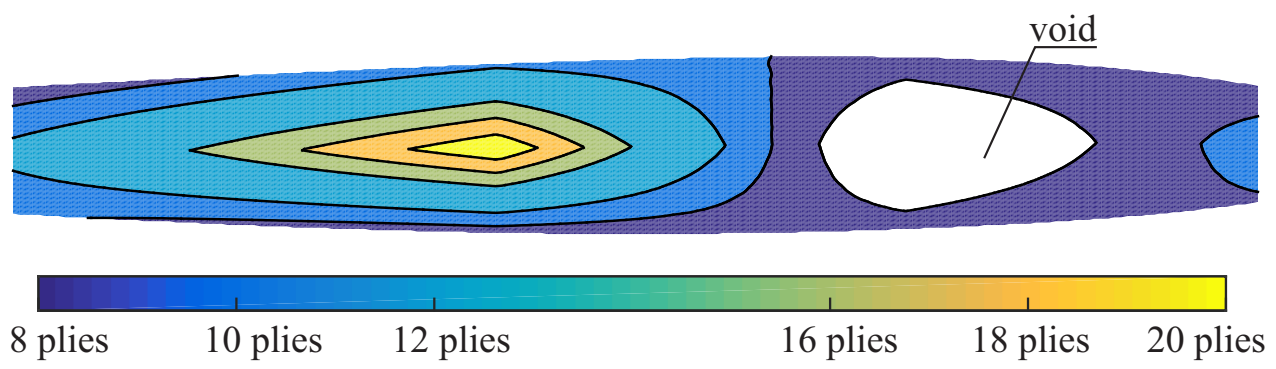

Figure 19: The contour plot of the thickness distribution and the topology at the optimized point where 12 design nodes are used.

The contour plot of the thickness distribution at the point of optimum is shown in Figure 19.

To be able to capture a more accurate design, the structure is also optimized with 21 design nodes. Figure 20 shows the distribution of the 21 design nodes. The evolution of the mass using 12 and 21 design nodes is shown in Figure 21.

As it can be seen in Figure 21 a smaller mass value is obtained using 21 design nodes. However, a larger number of design nodes resulted in more iterations before convergence as can be seen in Figure 21. Also, using 21 design nodes, it requires more computation cost related to the sensitivity analysis in each iteration. In particular, solving the problem using 21 design nodes required 350 buckling calculations while

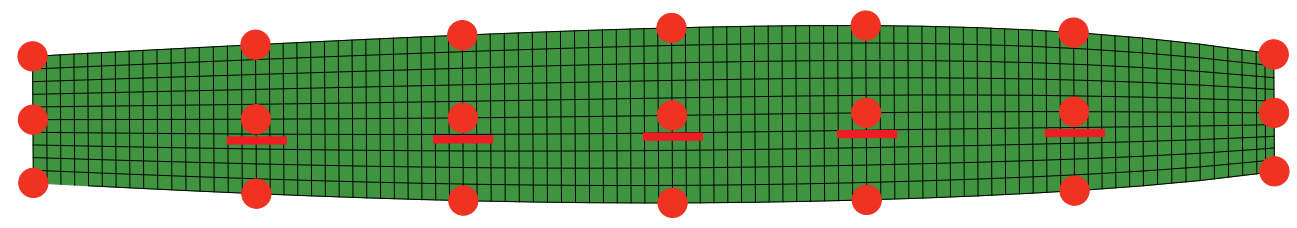

Figure 20: The distribution of the 21 design nodes. The design nodes that are allowed to obtain values smaller than the value of the first level are indicated with an underline. 


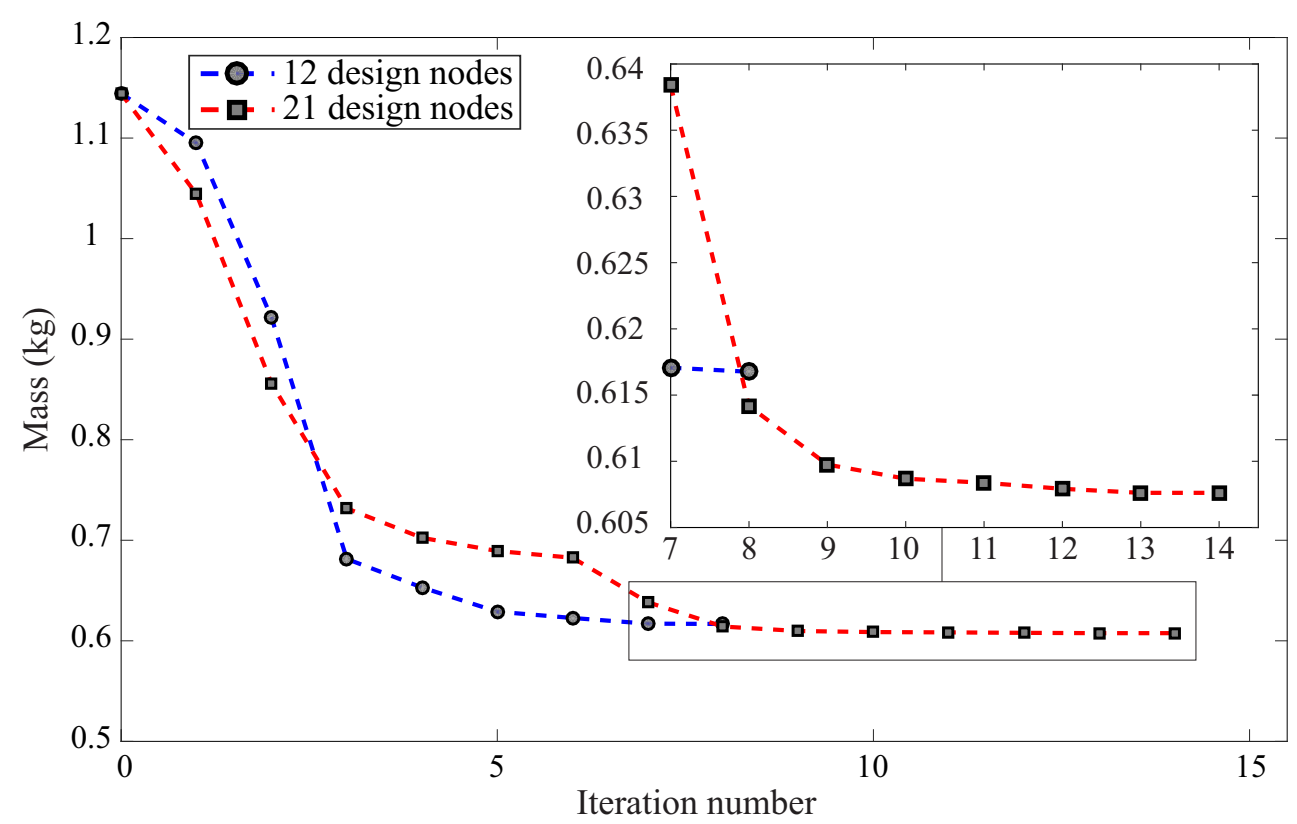

Figure 21: The evolution of the rib mass using 12 and 21 design nodes.

solving the problem with 12 design nodes required 128 buckling calculations. To reduce the overall computation cost while reaching a structure as light as that obtained using 21 design nodes, the design of the optimization problem with 12 design nodes after 4 iterations (where the obtained design is close to the optimum) was used as the initial design of the problem with 21 design nodes. As mentioned in Example 1, an initial design closer to the optimum, requires less iterations before convergence. Figure 22 shows the resulting mass evolution. Six additional iterations are performed with 21 design nodes before convergence. In Table 4 the overall number of function evaluations (related to the sensitivity analysis) for the optimization problem with 21 design nodes is compared for two cases: Case 1, a laminate with 18 plies is used as the initial design of the problem with 21 design nodes, Case 2, a laminate with 18 plies is used as the initial design of the problem with 12 design nodes, after 4 iterations the design is used as the initial design of the optimization problem with 21 design nodes. As it can be seen in Table 4, compared to Case 1, 124 fewer function evaluations were required until convergence in Case 2 . This significantly improves the efficiency of the proposed method in terms of computation cost.

The obtained mass using 12 and 21 design nodes is compared in Table 5. According to the table, the structural mass can be further reduced by $1.8 \%$ when 21 design nodes are used instead of 12 design nodes. The resulting contour plot of thickness distribution obtained using 21 design nodes is shown in Figure 23. As it can be seen in Figure 23 the hole emerges in the region where buckling does not occur.

It should be noted that the topology in the design is obtained without any predefined void. This stands in contrast to the conventional level-set based topology optimization 
Table 4: A comparison between the total number of iterations and the overall number of function evaluations (related to sensitivity analysis) for Case 1 and Case 2.

Case 1 Case 2

\begin{tabular}{ccc}
\hline Iterations with 12 design nodes & 0 & 4 \\
Iterations with 21 design nodes & 14 & 6 \\
Total number of iterations before convergence & 14 & 10 \\
Number of function evaluations & 308 & 184 \\
\hline
\end{tabular}

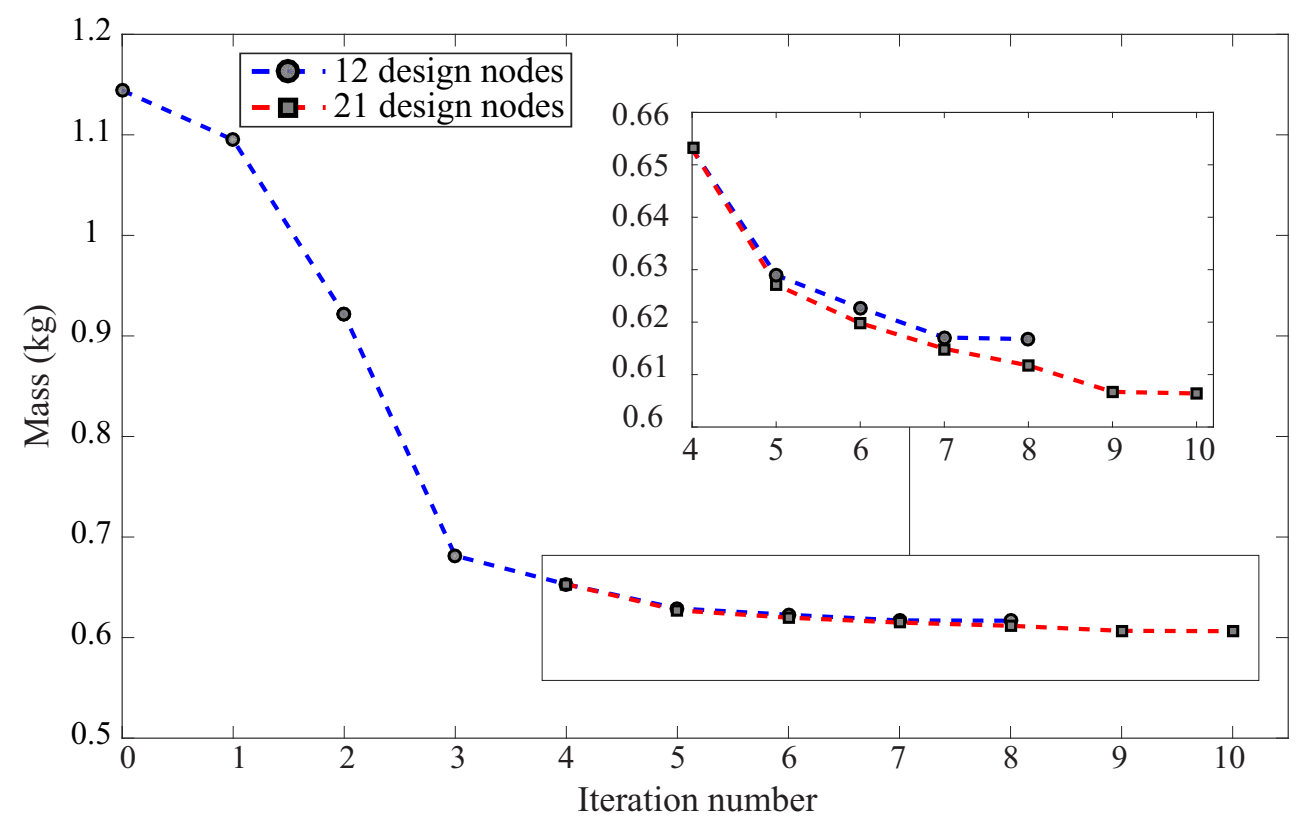

Figure 22: The design obtained in the $4^{\text {th }}$ iteration of the optimization problem with 12 design nodes is used as the initial design of the optimization problem with 21 design nodes.

Table 5: The mass obtained using the proposed method with 12 and 21 design nodes. 12 design nodes 21 design nodes

\begin{tabular}{lcc}
\hline Mass $(\mathrm{kg})$ & 0.617 & 0.606 \\
\hline
\end{tabular}



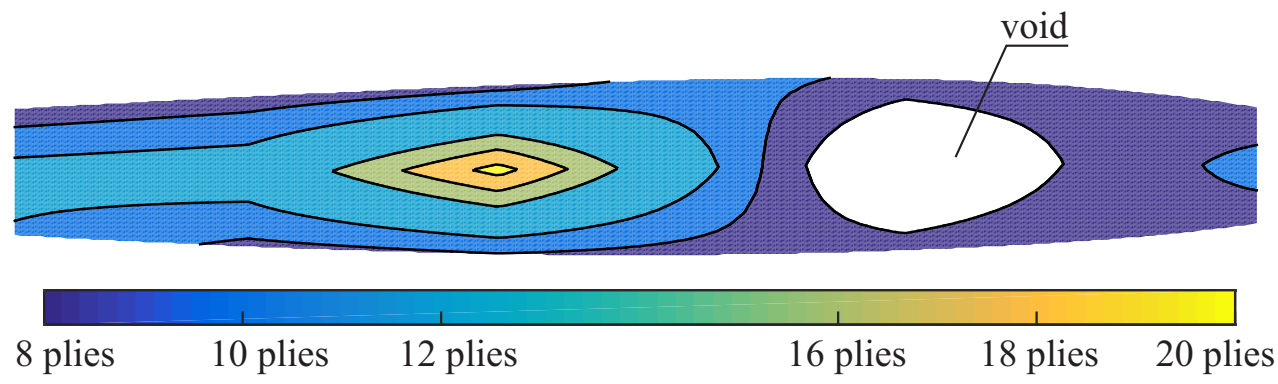

Figure 23: Contour plot of the optimized topology and thickness distribution for the optimization problem solved using 21 design nodes.

where topological derivatives of infinitesimal voids are required for the emergence of voids in the design.

\section{Summary and conclusion}

A method for the simultaneous optimization of the thickness, the topology, and the shape of a composite structure is presented in this work. All design, manufacturing, and strength related guidelines are satisfied using the proposed method. This is accomplished by developing a fully gradient based method that is capable of handling all the aforementioned guidelines using a simple concept.

An SST (Stacking Sequence Table) with optimized stacking sequences has to be generated first. As long as laminates in a design are selected from the SST, it is guaranteed that the design and the manufacturing guidelines are fulfilled. Next, the distribution of the laminates in the SST is determined by an auxiliary level-set function (LSF) using the proposed method. The LSF is defined through the interpolation of the values of a limited number of design nodes. The design node values can change continuously while optimizing the topology, shape, and thickness distribution. The continuous change of the design node values allows the application of any gradient based optimization algorithm to iteratively update the design.

To verify the proposed method, it is applied to the compliance minimization problem of a clamped plate. The clamped plate example was previously studied by Sørensen and Lund [16]. The shape and the thickness optimization using the proposed method are shown in this example. The results show a comparable thickness distribution for a less compliant design.

The proposed method is then applied to the mass minimization problem of a torsion box rib where buckling is the constraint of the problem. The capability of the proposed method in prescribing topology in the design as well as thickness distribution is examined in this example where lightening holes emerge in the design.

Based on the results from this example, it can be concluded that the proposed method can be efficiently applied to the optimization problem of a composite structure where 
several design, manufacturing, and strength related guidelines are required.

\section{Acknowledgements}

The support of this research by partners in TAPAS2 project is gratefully acknowledged.

\section{Appendix A. The procedure of generation an SST}

A 2-step method was presented by the authors to generate an SST where the fiber orientations have to be selected from a limited set of angles [36]. The (modified) two step procedure is described in the following:

\section{Step 1: obtaining the optimized stiffness and thickness distri- bution (idealized design)}

To generate an SST, first the idealized stiffness and thickness distribution are obtained [41]. To this end, the smeared stiffness method [42] is used to estimate the extensional (A) and the bending (D) stiffness matrices without knowing the stacking sequence of a laminate. This is achieved by assuming a homogeneous section for the layups [42]. According to the classical laminate theory [43], the matrix $\mathbf{A}$ is defined as:

$$
\mathbf{A}=h\left(\sum_{k=1}^{N} \frac{\left(\bar{Q}_{i j}\right)_{k}}{N}\right), \quad i=j=1,2,6
$$

where $N$ and $h$ represent the total number of plies and the total thickness of the laminate, respectively. $\bar{Q}_{i j}$ represents the transformed plane stiffness [43].

Using the material homogeneity assumption [42], the bending stiffness matrix (D) can be obtained through:

$$
\mathbf{D} \approx \mathbf{A} h^{2} / 12
$$

Liu et al. [42] proposed solving the following optimization problem to obtain an estimation about the idealized thickness and stiffness distribution over the structure:

$$
\min f=\sum_{j=1}^{N_{\mathrm{p}}}\left(n_{0}^{j}+n_{45}^{j}+n_{90}^{j}\right) S_{j} t
$$

where $N_{\mathrm{p}}$ is the total number of panels, $S_{j}$ is the area of panel $j$, and $t$ is the ply thickness. The number of plies of each orientation in each panel (or a set of panels), $n_{0}^{j}, n_{45}^{j}, n_{90}^{j}, j=1, \ldots, N_{\mathrm{p}}$ are the design variables of this optimization problem. Due to the assumed balance guideline, the number of $+45^{\circ}$ plies has to be equal to the number of $-45^{\circ}$ plies. Therefore, $n_{45}^{j}$ is defined to be the sum of $+45^{\circ}$ and $-45^{\circ}$ plies. 
In the present work, the constraints of the optimization problem in Equation (15) are defined as:

$$
\begin{array}{cl}
g_{i} \leq 0 & i=1 \text { to } N_{\mathrm{c}} \\
n_{0}^{j} / N^{j} \geq 0.1 & j=1 \text { to } N_{\mathrm{p}} \\
n_{45}^{j} / N^{j} \geq 0.2 & j=1 \text { to } N_{\mathrm{p}} \\
n_{90}^{j} / N^{j} \geq 0.1 & j=1 \text { to } N_{\mathrm{p}}
\end{array}
$$

where $g_{i}$ is a buckling constraint (defined in Section 3.2), $N_{\mathrm{c}}$ represents the number of required buckling constraints, and $N^{j}=n_{0}^{j}+n_{45}^{j}+n_{90}^{j}$. In Equation (16), the percentage of the plies of each orientation is required to be $\geq 10 \%$ in each panel.

As a result of solving the optimization problem defined in Equation (15) an estimation about the 'idealized' stiffness and thickness distribution will be obtained. The output of this step is a table called SST-data. In an SST-data table, each thickness value appears once and a unique $\mathbf{A D}$ stiffness vector is assigned to every (rounded to the nearest integer) thickness value.

\section{Step 2: fitting the stacking sequences}

In the second step, the stacking sequence of laminates with different thickness values is generated using the SST-data table obtained in step 1. In the procedure of generating the stacking sequences, all required laminate design guidelines have to be satisfied.

All valid laminates (laminates that satisfy the strength related guidelines) with the thickness equal to that of the thinnest laminate obtained in the step 1 are generated (see Figure 24 for details on this procedure).

Among the set of the valid thinnest laminates, the one which has the closest stiffness values to those estimated for the same thickness laminate in step 1, is selected. A Root Mean Square Error (RMSE) of the components of the $\mathbf{A}$ and the $\mathbf{D}$ matrices was used to identify the laminate with the desired stiffness values.

To generate a thicker laminate, a ply (or two plies in case the fiber orientations are $-45^{\circ}$ and $+45^{\circ}$ ) has to be added to a thinner laminate. All valid laminates, according to the required guidelines, are generated. From the set of the newly built laminates the one with stiffness values closest to those of the laminate with the same thickness in the SST-data table is selected. This procedure continues until a laminate with the thickness equal to that of the thickest laminate in the SST-data table is reached.

It is possible that as a result of adding a ply to a thinner laminate, the thickness of the newly built laminates does not exist among the thickness values in the SST-data table. In this case, the stiffness values of the laminate with the closest thickness values in the SST-data table are (linearly) interpolated and used to select the fittest laminate. Figure 24 shows the flowchart of the 2 -step procedure of generating an SST. 


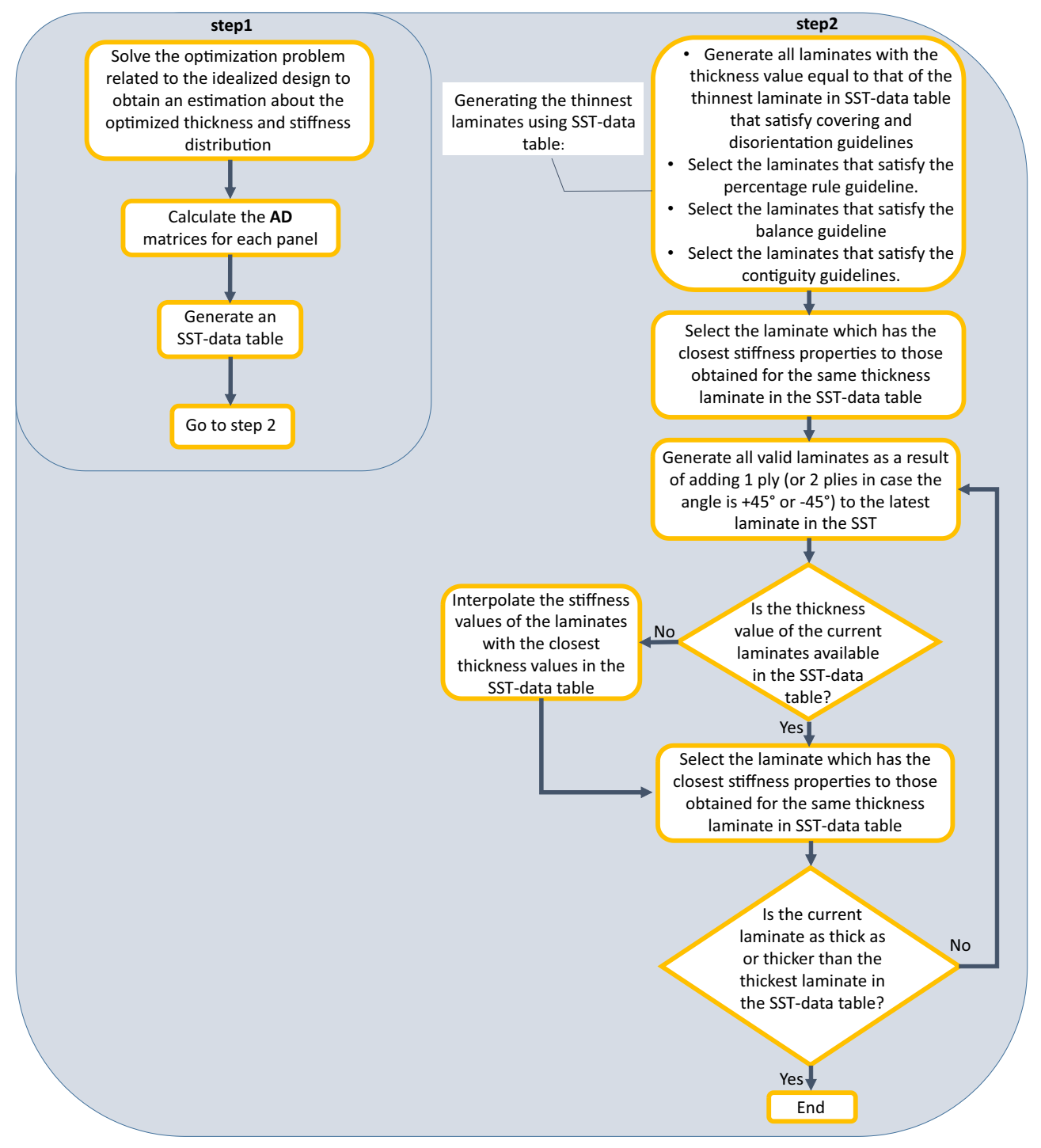

Figure 24: The flowchart of the 2-step procedure of generating an SST. 


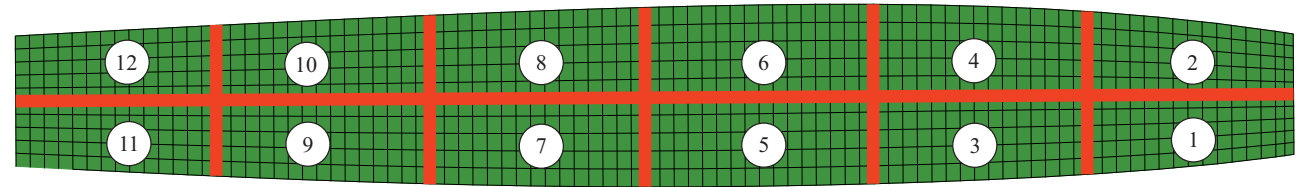

Figure 25: The discretization and the panel numbering of the rib studied in Example 2 in Section 4.

Table 6: The result of the optimization problem defined in Equation (15).

\begin{tabular}{ccccc}
\hline Panel & $n_{0}$ & $n_{45}$ & $n_{90}$ & $\begin{array}{c}\text { Idealized number of plies (rounded) } \\
2\left(n_{0}+n_{45}+n_{90}\right)\end{array}$ \\
\hline 1 & 1 & 2 & 1 & 8 \\
2 & 1 & 2 & 1 & 8 \\
3 & 1 & 2 & 1 & 8 \\
4 & 1 & 2 & 1 & 8 \\
5 & 1 & 2 & 1 & 8 \\
6 & 1 & 2 & 1 & 8 \\
7 & 1 & 2 & 3.75 & 14 \\
8 & 1 & 2 & 3.18 & 14 \\
9 & 1 & 2 & 3.98 & 14 \\
10 & 1 & 2 & 3.73 & 12 \\
11 & 1 & 2.17 & 2.48 & 10 \\
12 & 1 & 2 & 1.6 & \\
\hline
\end{tabular}

\section{Appendix B. Generating an SST for the rib presented in Example 2}

According to the step 1 procedure shown in Figure 24, the optimization problem defined in Equation (15) was solved using the MATLAB optimization toolbox. To solve this optimization problem, the rib was discretized into 12 panels. Figure 25 shows the discretization and the panel numbering.

The sensitivity of the objective function was calculated according to:

$$
\frac{\mathrm{d} f}{\mathrm{~d} n_{r}^{j}}=S_{j} t, \quad j=1 \text { to } N_{\mathrm{p}}, \quad r=0,45,90
$$

where $n_{r}^{j}$ represents the design variable related to the ply with fiber orientation $r$, for the $j^{\text {th }}$ panel. $S_{j}$ and $t$ represent the area of the $j^{\text {th }}$ panel and the ply thickness, respectively. $N_{\mathrm{p}}$ represents the total number of panels.

A numerical forward difference scheme (as defined in 3.2) was performed to calculate buckling constraint sensitivities defined in Equation (16).

Table 6 shows the result of the optimization problem defined in Equation (15).

As seen in Table 6, panels 7 to 10, which are under a larger compressive load (see Figure 16), obtained thicker idealized laminates. The majority of the plies in the laminates of panels 7 to 10 have $90^{\circ}$ fiber orientation. This is due to the fact that the compressive load is in the $y$ direction. Using the information obtained from solving 
Equation (15) and the fitting procedure described in Appendix A, the SST shown in Figure 1 is obtained.

The idealized design gives a constant thickness value per panel. Using the proposed level-set method, however, each panel may include various thickness values. The area weighted average of various thicknesses in each panel is expected to be close to the idealized thickness value obtained for each panel. Therefore, as a result of the level-set-based thickness optimization, laminates thicker and thinner than the idealized laminate are also expected in each panel. For this reason, the SST shown in Figure 1 includes thicker laminates compared to those obtained in the idealized design (see Table 6) to avoid unnecessarily restricting the design variables [36]. The AD stiffness values of the thickest and the thinnest laminates in the idealized design were extrapolated to estimate the stiffness values of laminates that are not suggested by the idealized design, but do exist in the SST. The extrapolated stiffness values were used in the selection procedure of the fittest laminate as discussed in Appendix A.

A seen in Table 6, a laminate with 14 plies is prescribed for panels 7, 9, and 10 . However, this thickness does not exist among the thickness values shown in 1 . The reason is that after generating a laminate with 12 plies, it was requires to add a $-45^{\circ}$ and $\mathrm{a}+45^{\circ}$ ply due to the percentage rule guideline. Therefore, in the SST shown in 1, the laminate thicker than 12 plies has 16 plies. 


\section{References}

[1] Military Handbook - MIL-HDBK-17-3F: Composite Materials Handbook, Volume 3 - Polymer Matrix Composites Materials Usage, Design, and Analysis. U.S. Department of Defense, 2002.

[2] J. A. Bailie, R. P. Ley, and A. Pasricha. A summary and review of composite laminate design guidelines. Technical report NASA, NAS1-1934\%. Northrop Grumman-Military Aircraft Systems Division, 1997.

[3] J. Yang, B. Song, X. Zhong, and P. Jin. Optimal design of blended composite laminate structures using ply drop sequence. Composite Structures, 135:30 - 37, 2016.

[4] Y. M. Meddaikar, F. X. Irisarri, and M. M. Abdalla. Laminate optimization of blended composite structures using a modified shepard's method and stacking sequence tables. Structural and Multidisciplinary Optimization, 55(2):535-546, 2017.

[5] G. Allaire and G. Delgado. Stacking sequence and shape optimization of laminated composite plates via a level-set method. Journal of the Mechanics and Physics of Solids, 97:168 - 196, 2016.

[6] M. P. Bendsøe. Optimal shape design as a material distribution problem. Structural optimization, 1(4):193-202, Dec 1989.

[7] M. Stolpe and K. Svanberg. An alternative interpolation scheme for minimum compliance topology optimization. Structural and Multidisciplinary Optimization, 22(2):116-124, Sep 2001.

[8] J. Stegmann and E. Lund. Discrete material optimization of general composite shell structures. International Journal for Numerical Methods in Engineering, 62(14):2009-2027, 2005.

[9] M. P. Bendsøe and N. Kikuchi. Generating optimal topologies in structural design using a homogenization method. Computer Methods in Applied Mechanics and Engineering, 71(2):197 - 224, 1988.

[10] G. I. N. Rozvany, M. Zhou, and T. Birker. Generalized shape optimization without homogenization. Structural optimization, 4(3):250-252, Sep 1992.

[11] O. Sigmund and S. Torquato. Design of materials with extreme thermal expansion using a three-phase topology optimization method. Journal of the Mechanics and Physics of Solids, 45(6):1037 - 1067, 1997.

[12] L. V. Gibiansky and O. Sigmund. Multiphase composites with extremal bulk modulus. Journal of the Mechanics and Physics of Solids, 48(3):461 - 498, 2000.

[13] J. Thomsen. Topology optimization of structures composed of one or two materials. Structural optimization, 5(1):108-115, Mar 1992. 
[14] C. F. Hvejsel and E. Lund. Material interpolation schemes for unified topology and multi-material optimization. Structural and Multidisciplinary Optimization, 43(6):811-825, Jun 2011.

[15] E. Lund and J. Stegmann. On structural optimization of composite shell structures using a discrete constitutive parametrization. Wind Energy, 8(1):109-124, 2005 .

[16] S. N. Sørensen and E. Lund. Topology and thickness optimization of laminated composites including manufacturing constraints. Structural and Multidisciplinary Optimization, 48(2):249-265, Aug 2013.

[17] S. N. Sørensen, R. Sørensen, and E. Lund. DMTO - a method for discrete material and thickness optimization of laminated composite structures. Structural and Multidisciplinary Optimization, 50(1):25-47, Jul 2014.

[18] E. Lund. Discrete material and thickness optimization of laminated composite structures including failure criteria. Structural and Multidisciplinary Optimization, pages 1-19, 2017.

[19] S. N. Sørensen and M. Stolpe. Global blending optimization of laminated composites with discrete material candidate selection and thickness variation. Structural and Multidisciplinary Optimization, 52(1):137-155, 2015.

[20] P. Duysinx and M. P. Bendsøe. Topology optimization of continuum structures with local stress constraints. International Journal for Numerical Methods in Engineering, 43(8):1453-1478, 1998.

[21] M. Stolpe and K. Svanberg. Modelling topology optimization problems as linear mixed 0-1 programs. International Journal for Numerical Methods in Engineering, 57(5):723-739, 2003.

[22] M. Bruggi. On an alternative approach to stress constraints relaxation in topology optimization. Structural and Multidisciplinary Optimization, 36(2):125-141, Aug 2008.

[23] N. P. Van Dijk, K. Maute, M. Langelaar, and F. Van Keulen. Level-set methods for structural topology optimization: A review. Structural and Multidisciplinary Optimization, 48(3):437-472, 2013.

[24] V. J. Challis. A discrete level-set topology optimization code written in matlab. Structural and Multidisciplinary Optimization, 41(3):453-464, 2010.

[25] J. Liu and Y. Ma. A new multi-material level set topology optimization method with the length scale control capability. Computer Methods in Applied Mechanics and Engineering, 329:444-463, 2018.

[26] L. A. Vese and T. F. Chan. A multiphase level set framework for image segmentation using the Mumford and Shah model. International Journal of Computer Vision, 50(3):271-293, 2002. 
[27] I. Baran, C. C. Tutum, and J. H. Hattel. Optimization of the thermosetting pultrusion process by using hybrid and mixed integer genetic algorithms. Applied Composite Materials, 20(4):449-463, Aug 2013.

[28] C. C. Tutum, I. Baran, and K. Deb. Optimum design of pultrusion process via evolutionary multi-objective optimization. The International Journal of Advanced Manufacturing Technology, 72(9):1205-1217, Jun 2014.

[29] B. Liu and R. T. Haftka. Composite wing structural design optimization with continuity constraints. In 19th AIAA Applied Aerodynamics Conference, March 2001.

[30] D. B. Adams, L. T. Watson, Z. Gürdal, and C. M. Anderson-Cook. Genetic algorithm optimization and blending of composite laminates by locally reducing laminate thickness. Advances in Engineering Software, 35(1):35-43, 2004.

[31] D. B. Adams, L. T. Watson, O. Seresta, and Z. Gürdal. Global/local iteration for blended composite laminate panel structure optimization subproblems. Mechanics of Advanced Materials and Structures, 14(2):139-150, 2007.

[32] N. Zehnder and P. Ermanni. A methodology for the global optimization of laminated composite structures. Composite Structures, 72(3):311 - 320, 2006.

[33] N. Zehnder and P. Ermanni. Optimizing the shape and placement of patches of reinforcement fibers. Composite Structures, 77(1):1 - 9, 2007.

[34] F. X. Irisarri, A. Lasseigne, F. H. Leroy, and R. Le Riche. Optimal design of laminated composite structures with ply drops using stacking sequence tables. Composite Structures, 107:559-569, 2014.

[35] A. Carpentier, L. Michel, S. Grihon, and J. J. Barrau. Buckling optimization of composite panels via lay-up tables. In 3rd European Conference on Computational Mechanics Solids, Structures and Coupled Problems in Engineering, Lisbon, Portugal, June 2006.

[36] F. Farzan Nasab, H. J. M. Geijselaers, I. Baran, and A. de Boer. Generating the best stacking sequence table for the design of blended composite structures. In Advances in Structural and Multidisciplinary Optimization: Proceedings of the 12th World Congress of Structural and Multidisciplinary Optimization (WCSMO12), pages 779-788, 2018.

[37] J. S. Arora. Introduction to optimum design. Academic Press, Boston, third edition, 2012.

[38] R. J. Allemang and D. L. Brown. A correlation coefficient for modal vector analysis. In 1st International Modal Analysis Conference, 1982.

[39] L. S. Cecchini and P. M. Weaver. Brazier effect in multibay airfoil sections. AIAA Journal, 43(10):2252-2258, 2005. 
[40] B. K. Stanford and P. D. Dunning. Optimal topology of aircraft rib and spar structures under aeroelastic loads. Journal of Aircraft, 52(4):1298-1311, 2015.

[41] F. X. Irisarri, D. M. J. Peeters, and M. M. Abdalla. Optimisation of ply drop order in variable stiffness laminates. Composite Structures, 152:791-799, 2016.

[42] D. Liu, V. V. Toroporov, O. M. Querin, and D. C. Barton. Bilevel optimization of blended composite wing panels. Journal of Aircraft, 48(1):107-118, 2011.

[43] J. N. Reddy. Mechanics of laminated composite plates : theory and analysis. Boca Raton : CRC Press, 1997. 



\section{Paper C}

\section{A gradient-based strategy for the optimization of stiffened composite structures subject to multiple load cases and multiple failure criteria}

This chapter is based on the paper 'A gradient-based strategy for the optimization of stiffened composite structures subject to multiple load cases and multiple failure criteria', presented at 'EngOpt 2018 Proceedings of the 6th International Conference on Engineering Optimization' (2018). 



\title{
A gradient-based strategy for the optimization of stiffened composite structures subject to multiple load cases and multiple failure criteria
}

\author{
F. Farzan Nasab ${ }^{1)}$, G.A. Duipmans ${ }^{1)}$, H.J.M. Geijselaers ${ }^{1)}$, A. de Boer ${ }^{1)}$
}

Presented at EngOpt 2018 Proceedings of the 6th International Conference on Engineering Optimization

${ }^{1)}$ Applied Mechanics, Engineering Technology, University of Twente, Enschede, The Netherlands

\begin{abstract}
This work aims at investigating the applicability of the level-set based thickness optimization method, earlier proposed by the authors, to a realistic structure. The design has to have sufficient stiffness and strength while the structural mass is minimized. The concerned composite structure is subjected to multiple load cases. The proposed method guarantees the fulfillment of the design guidelines, namely symmetry, covering ply, disorientation, percentage rule, balance, and contiguity of the layup. The stiffeners divide a composite structure into several smaller panels. The manufacturability of a resulting design is guaranteed as plies are continuous among adjacent panels (the design is blended). The proposed method is successfully applied to the mass minimization problem of the stiffened top and bottom skin of a wing torsion box. The structure, subject to two load cases, is optimized where local buckling and allowable strain are the constraints of the problem.
\end{abstract}




\section{Introduction}

A composite structure design procedure requires the determination of the total number of plies and the order of fiber orientation as design variables and satisfaction of several strength related guidelines as constraints. Due to the large number of design variables and constraints, the optimization of composite structures is a highly challenging problem. For large scale structures, stiffeners are added to enhance the structural performance in carrying loads. Stiffeners divide the structure into smaller panels. It is important that the plies are continuous (blended) in adjacent panels. This ensures manufacturability of the composite structure. Manufacturability of the composite structure is a particularly difficult constraint to deal with [1]. Significant manufacturing difficulties appear when panels are designed individually [2-4]. This is due to the considerable difference in the resulting stacking sequence of the laminates in adjacent panels.

A large amount of research has been dedicated to the optimization of composite structures considering blending (see e.g [4-7]). An efficient method to provide globally blended design, is the use of the Stacking Sequence Table (SST) introduced by Carpentier et al. [8]. In [8], the order of ply drop from a thickest laminate is determined. In [9] the genetic algorithm (GA) is used to optimize SSTs. Irissari et al. [10] investigates the application of topology optimization together with a GA-based generated SST to obtain a more accurate ply drop locations over the structure. However, the application of a GA for the optimization of a real-scale structure is not practical as a GA typically requires a large number of function evaluation [11].

A gradient based strategy has been introduced by the authors to provide a fully blended optimized structures [12]. To this end, first an SST has to be generated. The laminates in an SST have to fulfill the required guidelines related to the design of a composite structure. An extensive discussion about these guidelines can be found in $[13,14]$. A detailed description on optimizing an SST has been published by the authors [15]. Then, a convenient level-set based method has been proposed to prescribe the optimized thickness distribution based on the provided SST [12]. The level-set based thickness optimization is fully gradient based. This method aims at turning the discrete nature of the optimization problem (caused by integer laminate thickness values) into a continuous problem.

The goal of the current research is to investigate the capability of the level-set based method, previously introduced by the authors [12], in dealing with the thickness optimization of structures subject to multiple load cases and multiple failure criteria. In particular, The top and the bottom skin of a wing torsion box are the target structure for optimization where the wing is subject to 2 distinct load cases. Local buckling and maximum strain are considered as failure criteria. The rest of this paper is organized as follows: the optimization procedure is briefly introduced in Section 2. In Section 3, the optimization results are presented and discussed. The work is concluded in Section 4. 


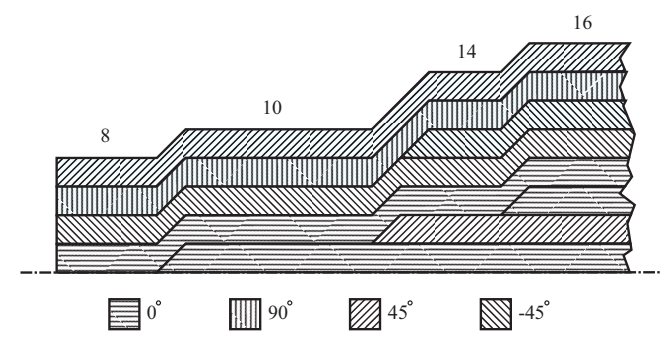

Figure 1: A blended structure where laminates are selected from the SST shown in Table 1.

\section{Optimization procedure}

\subsection{Stacking sequence table (SST)}

An SST prescribes the stacking sequence of a set of laminates. A thinner laminate in an SST is obtained by selectively dropping plies from a thicker one. This guarantees a blended final design as long as laminates are selected from the SST. Any laminate in an SST has to satisfy several guidelines related to the design of composite structures. These guidelines are mainly based on the industrial experience and help to avoid failure modes such as delamination. The following guidelines are considered in the current research [9]:

- Symmetry, the laminate should be symmetric with respect to its center line.

- Balance, the total number of plies with $+45^{\circ}$ orientation in a laminate is equal to the total number of plies with $-45^{\circ}$ orientation.

- Covering plies, the outermost ply has to have the orientation of either $+45^{\circ}$ or $-45^{\circ}$.

- Disorientation, the maximum orientation difference of each two adjacent plies is $45^{\circ}$.

- Contiguity, not more than a certain number of successive plies with a same orientation are allowed to stack together.

- Percentage rule, the number of plies of a certain orientation with respect to the total number of plies in a laminate must fall within a specified range.

Here, it is assumed that the density of ply fibers and the ply thickness are constant. Also the ply orientations are limited to be selected from the set $\left\{0^{\circ}, \pm 45^{\circ}\right.$, and $\left.90^{\circ}\right\}$ of angles. An SST, as an example, is shown in Table 1. In this table, each column represents the stacking sequence of a laminate. Note that all laminates are symmetric and only the upper half is shown in the table. Figure 1 shows a blended structure as a result of selecting the laminates from the SST shown in Table 1. 
Table 1: A sample SST. Due to symmetry, the stacking sequence of half laminates are shown.

\begin{tabular}{|c|c|c|c|c|c|c|c|c|c|c|}
\hline Ply number & 8 & 10 & 14 & 16 & 18 & 22 & 24 & 26 & 30 & 32 \\
\hline \multirow{9}{*}{ 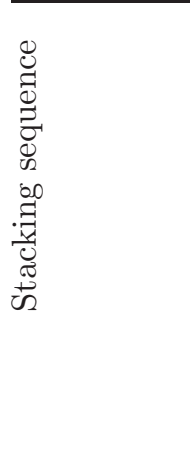 } & 45 & 45 & 45 & 45 & 45 & 45 & 45 & 45 & 45 & 45 \\
\hline & 90 & 90 & 90 & 90 & 90 & $\begin{array}{r}90 \\
-45\end{array}$ & $\begin{array}{r}90 \\
-45\end{array}$ & $\begin{array}{r}90 \\
-45\end{array}$ & $\begin{array}{r}90 \\
-45\end{array}$ & $\begin{array}{r}90 \\
-45\end{array}$ \\
\hline & & & -45 & -45 & -45 & -45 & -45 & -45 & -45 & -45 \\
\hline & & & & & 90 & 90 & 90 & 90 & $\begin{array}{r}90 \\
-45\end{array}$ & $\begin{array}{r}90 \\
-45\end{array}$ \\
\hline & -45 & -45 & -45 & -45 & -45 & -45 & -45 & -45 & -45 & $\begin{array}{r}90 \\
-45\end{array}$ \\
\hline & 0 & 0 & & 0 & 0 & 0 & 0 & & 0 & 0 \\
\hline & & & 45 & 45 & 45 & 45 & 45 & 45 & 45 & 45 \\
\hline & & 0 & 0 & 0 & 0 & 0 & 0 & 0 & 0 & 0 \\
\hline & & & & & & 45 & 45 & 45 & 45 & 45 \\
\hline
\end{tabular}

Generating an SST only based on the aforementioned design guidelines results in multiple SSTs. A method has been developed by the authors to generate a unique SST with optimized stacking sequences [15].

\subsection{Thickness optimization}

An optimization problem in the following form has to be solved to obtain the locations of the ply drops over the structure:

$$
\begin{aligned}
\min & f(X) \\
\text { subject to } & g_{i}<=0 \\
& x_{\min }<x<x_{\max }
\end{aligned} \quad i=1, \ldots, N_{\mathrm{c}}
$$

where $f$ and $g_{i}$ represent the objective and the $i^{\text {th }}$ constraint of the optimization problem, respectively. $X$ is the vector of design variables. $x_{\min }$ and $x_{\max }$ represent a lower bound and an upper bound for each design variable, respectively. The values of the design variables represent laminate thicknesses with stacking sequences from the optimized SST. The design variables form a level-set function which prescribes the ply drop locations over the structure [12].

In particular, local buckling and strain constraints are studied in this research. The following eigenvalue equation gives the buckling factors:

$$
\left(\mathbf{K}_{\mathrm{B}}-\lambda_{i} \mathbf{K}_{\mathrm{G}}\right) \cdot w_{i}=0
$$

where $\mathbf{K}_{\mathrm{B}}$ and $\mathbf{K}_{\mathrm{G}}$ represent the global bending stiffness matrix and the global geometric stiffness matrix, respectively. Solving Equation (2) gives a number of eigenvalues $\lambda$ equal to the rank of $\mathbf{K}_{\mathrm{G}}$. The vector $w_{i}$ is the mode shape corresponding to the $i^{\text {th }}$ buckling factor. As for the strain constraints, the in-plane principal strains are considered to be compared to their allowable values. Numerical forward difference scheme is used to calculate the sensitivity of the objective function and the constraints defined in Equation (1). 


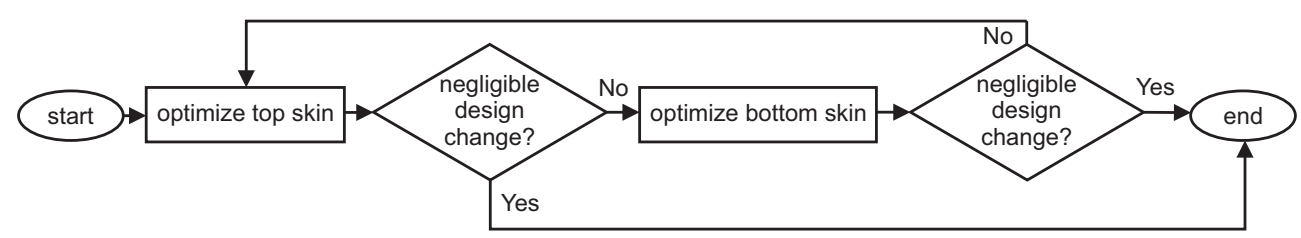

Figure 2: The two-level optimization work flow, adopted to optimize the top and the bottom skin of a wing.

\section{Results and discussion}

The target structure for optimization is the top and the bottom skin of a wing torsion box. Here, the following optimization problem is solved to obtain the thickness distribution over the structure:

$$
\begin{array}{rlrl}
\min & f(X)=W_{\text {top skin }}+W_{\text {bottom skin }} & \\
\text { subject to } & 1-\lambda_{i}^{k}<=0 & i=1, \ldots, N_{\lambda}, k=1,2 \\
& \varepsilon^{k}-\varepsilon_{\text {all }}<=0 & k=1,2 \\
& x_{\min }<x<x_{\max } &
\end{array}
$$

where $W_{\text {top skin }}$ and $W_{\text {bottom skin }}$ represent the weight of the top and the bottom skin, respectively. $\lambda_{i}^{k}$ represents the $i^{\text {th }}$ buckling factor of the structure (see Equation 2) when it is subjected to the $k^{\text {th }}$ load case, and $\varepsilon_{\text {all }}$ is the allowable strain.

To calculate the buckling factor and principal strains, ABAQUS 6.12 finite element package is used. The used finite element model returns the strains for each element. Therefore, the 2D principal strains are compared to the allowable strain for each finite element belonging to the either of the skins. The following definition is used for the strain constraints:

$$
\begin{aligned}
& \varepsilon_{\max }^{(j)} \leq \varepsilon_{\mathrm{T}}, \\
& \varepsilon_{\min }^{(j)} \geq \varepsilon_{\mathrm{C}},
\end{aligned}
$$

where:

$\varepsilon_{\max }^{(j)}$ the maximum principal strain in element $j$,

$\varepsilon_{\min }^{(j)}$ the minimum principal strain in element $j$,

$\varepsilon_{\mathrm{T}}$ the allowable tensile strain, and

$\varepsilon_{\mathrm{C}}$ the allowable compressive strain.

To optimize the top and the bottom skin, a two-level optimization procedure is adopted. In the first level the top skin, and in the second level the bottom skin is optimized. The design of the either of the skins affects the load applied to the other skin and thus affects the design of the other skin. Therefore, the two levels of the optimization procedure are related to each other through the loads applied to each skin. Figure 2 shows this two-level optimization procedure. 


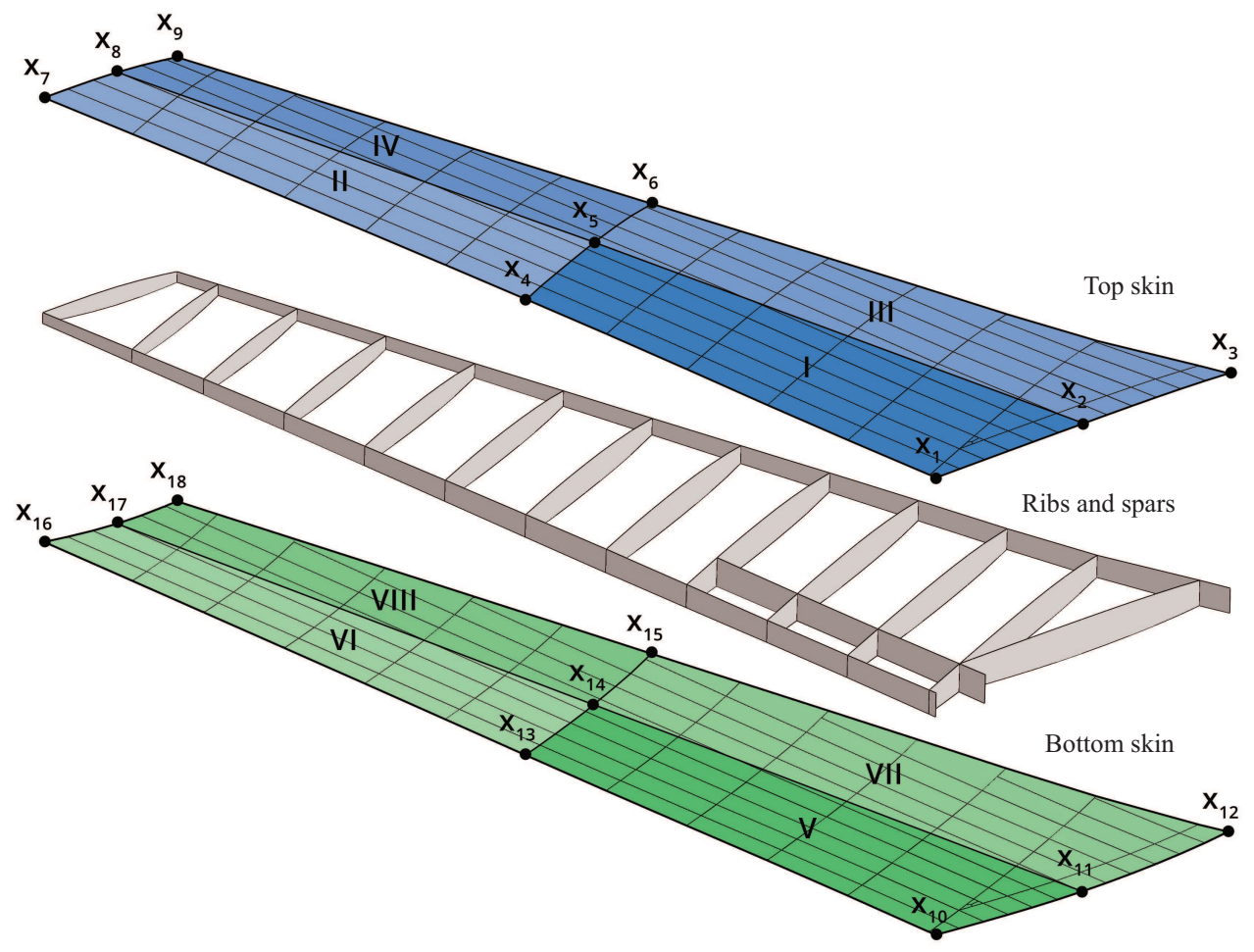

Figure 3: The distribution of the location of design variables. The interpolation areas are denoted by Roman numbers.

Here, 9 design variables are assigned to each skin. The distribution of the location of the design variables is shown in Figure 3. In this figure, the interpolation areas are denoted by Roman numbers.

The concerning wing is analyzed with 2 load cases. Figure 4 shows the deflection of the wing with respect to each load case when both skins are covered with 24 plies with stacking sequences from Table 1 . As it can be seen in the figure, load case 1 mainly causes upward bending while load case 2 results in a torsion in the wing. As a result of this analysis, the loads applied to each skin are extracted. Figure 5 shows the loads applied to the skins. Arrows in the figure represent shear load in the direction of the arrow.

The optimized contour plot of the top and the bottom skin is shown in Figure 6 . As it can be seen in the figure, the root of the top skins is covered with relatively thicker laminates. This is expected as the root is subjected to a larger compressive shear load compared to the tip.

The progress of the optimization objective is shown in Figure 7. In this figure, the part of the optimization related to the top skin is distinguished from that related to the optimization of the bottom skin. As it can be seen in Figure 7, it takes 52 


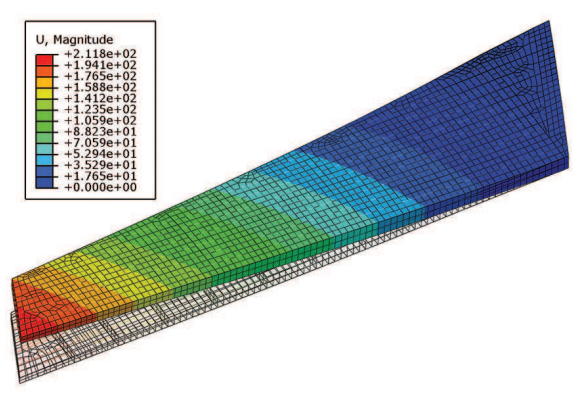

(a) Load case 1

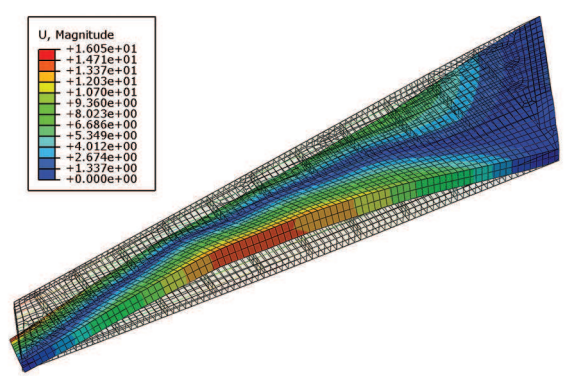

(b) Load case 2

Figure 4: The wing deflection as a result of 2 distinct load cases when both skins are covered with 24 plies with the stacking sequences from Table 1 . The deflections are plotted with a deformation scale of 2.6 for load case 1 , and 34.8 for load case 2 . The undeformed shape is shown in black and white.

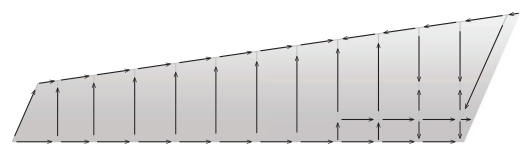

(a) Top skin, load case 1

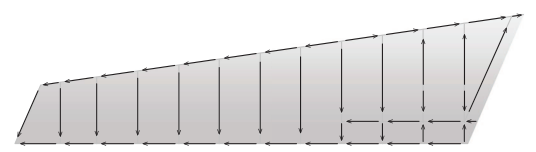

(c) Bottom skin, load case 1

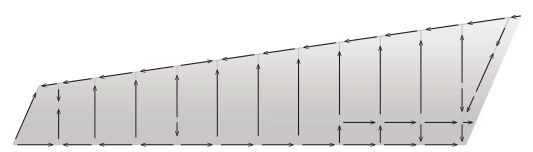

(b) Top skin, load case 2

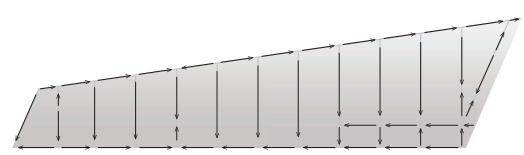

(d) Bottom skin, load case 2

Figure 5: The extracted shear loads applied to the wing skins as a result of the two load cases. 


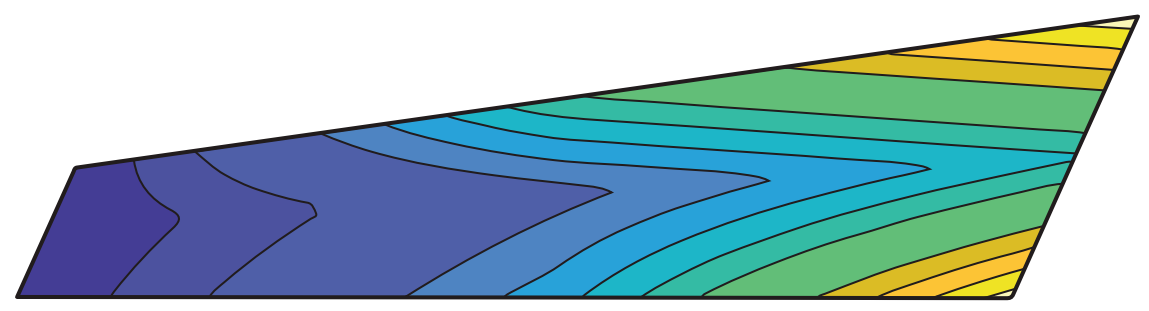

Top skin

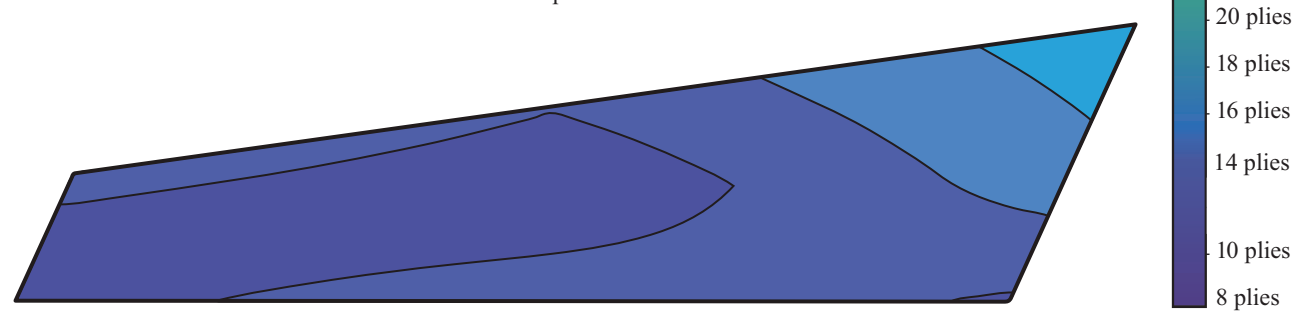

Bottom skin

Figure 6: The optimized contour plot of the top and the bottom skin where the wing is subjected to two load cases shown in Figure 5. The stacking sequence of the laminates is selected from Table 1 .

iterations before the optimization problem converges; however, after 27 iterations the objective progress is negligible.

The normalized maximum and minimum principal strains for each finite element at the optimized design is shown in Figure 8. As it can be seen in this figure, the maximum (tensile) strain constraint is at the critical value for the bottom skin as a result of load case 1 . This is expected as load case 1 causes upward bending in the wing, thus the bottom skin is subjected to tensile loads.

Figure 9 shows the buckling factors of the top and the bottom skin at the optimum design. As it can be seen in this figure, the buckling factor of the top skin is at the critical value for load case 1. From Figure 8 and Figure 9, it can be concluded that the design of the top skin is mainly driven by the buckling constraint while the bottom

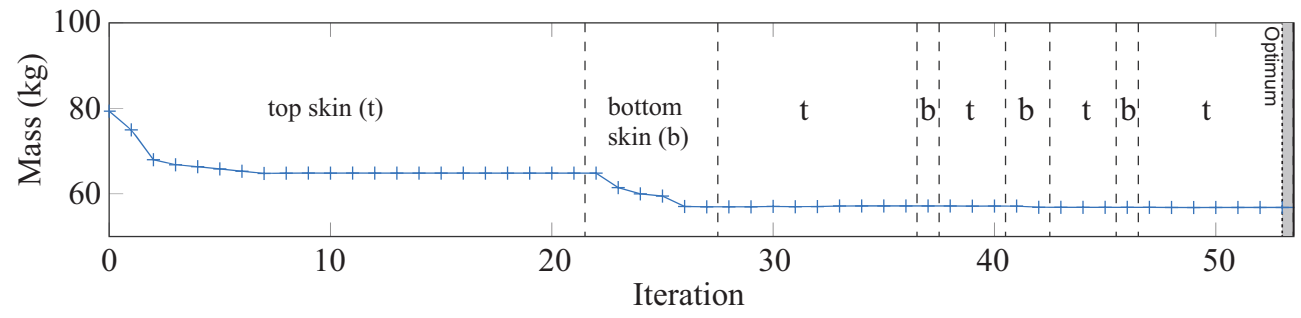

Figure 7: The progress of the two-level optimization objective. The part of the optimization related to the top skin is distinguished from that related to the optimization of the bottom skin. 


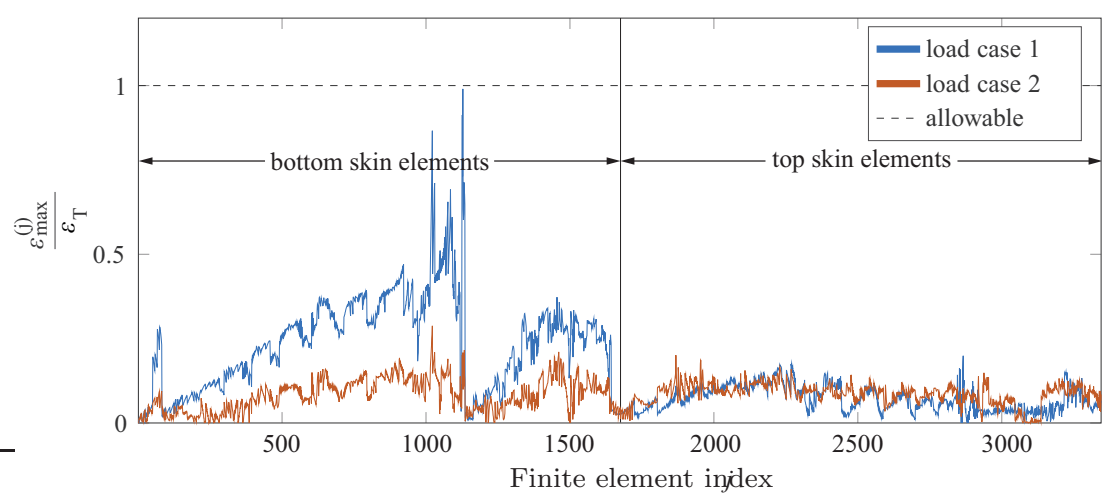

(a) Normalized maximum principal strains

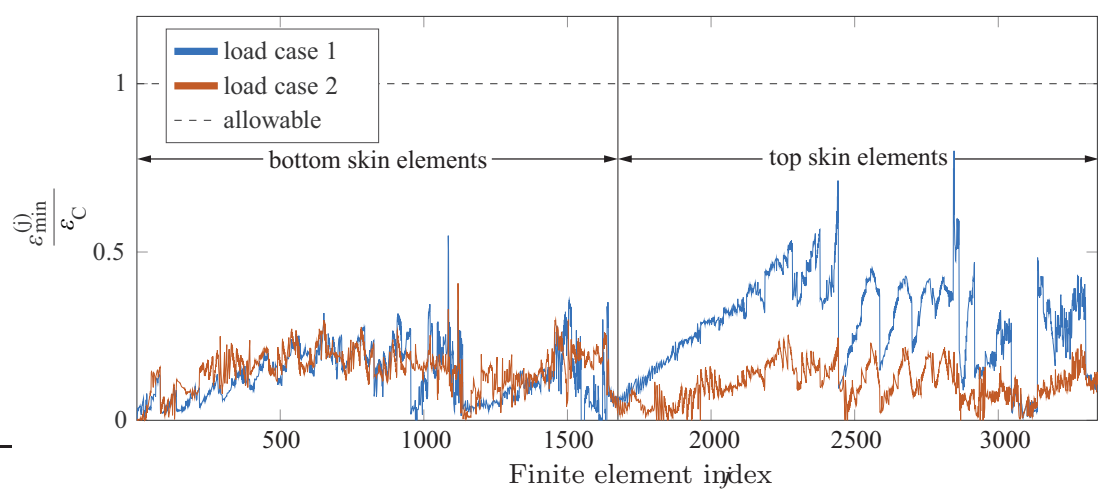

(b) Normalized minimum principal strains

Figure 8: The normalized maximum and minimum principal strains for each finite element at the optimized design. The horizontal axis shows the index for each finite element. 


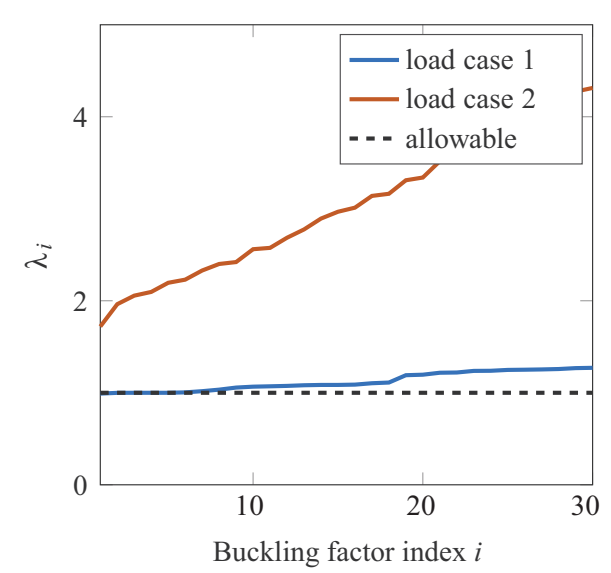

(a) Top skin buckling factor

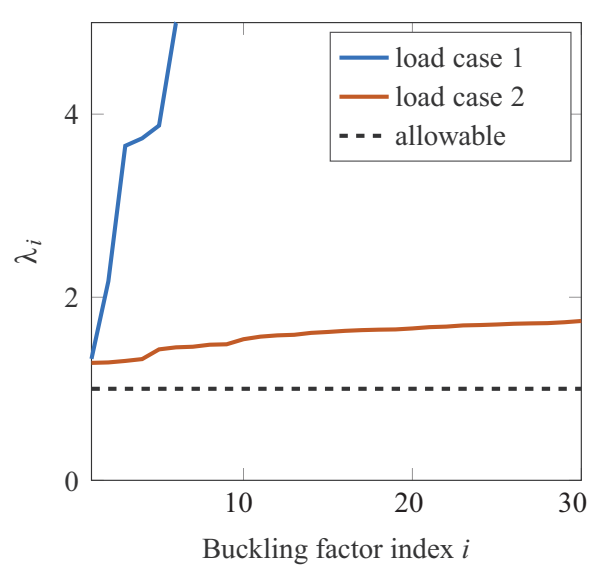

(b) Bottom skin buckling factor

Figure 9: The buckling factor for the top and the bottom skin at the optimized design. The horizontal axis shows the index of the buckling factor.

skin design is mainly driven by the strain constraint.

\section{Conclusion}

The present research aims at investigating the applicability of the level-set based thickness optimization method, proposed by the authors [12], to a realistic structure. Two load cases and two strength related failure criteria were considered. In particular, local buckling and strain were considered as the constraints of the optimization problem. The final design has to satisfy several other guidelines related to the design of the composite structures. To guarantee the manufacturability of the final design laminates have to be blended.

In this study, it is shown that the method proposed by the authors (which includes SST optimization [15], and thickness optimization [12]) can optimize the top and the bottom skin of a wing torsion box while the wing is subjected to two distinct load cases. The whole optimization process is performed at a limited computation cost. This makes the proposed method to be applicable in practice and stands in contrast to the expensive GAs widely used for the optimization of composite structures.

\section{Acknowledgements}

The support of this research by partners in TAPAS2 project is gratefully acknowledged. 


\section{References}

[1] G. Allaire and G. Delgado. Stacking sequence and shape optimization of laminated composite plates via a level-set method. Journal of the Mechanics and Physics of Solids, 97:168 - 196, 2016.

[2] M. T. McMahon and L. T. Watson. A distributed genetic algorithm with migration for the design of composite laminate structures. Parallel Algorithms and Applications, 14:329-362, 2000.

[3] G. Soremekun, Z. Gürdal, C. Kassapoglou, and D. Toni. Stacking sequence blending of multiple composite laminates using genetic algorithms. Composite Structures, 56(1):53-62, 2002.

[4] D. B. Adams, L. T. Watson, Z. Gürdal, and C. M. Anderson-Cook. Genetic algorithm optimization and blending of composite laminates by locally reducing laminate thickness. Advances in Engineering Software, 35(1):35-43, 2004.

[5] D. B. Adams, L. T. Watson, O. Seresta, and Z. Gürdal. Global/local iteration for blended composite laminate panel structure optimization subproblems. Mechanics of Advanced Materials and Structures, 14(2):139-150, 2007.

[6] N. Zehnder and P. Ermanni. A methodology for the global optimization of laminated composite structures. Composite Structures, 72(3):311 - 320, 2006.

[7] N. Zehnder and P. Ermanni. Optimizing the shape and placement of patches of reinforcement fibers. Composite Structures, 77(1):1 - 9, 2007.

[8] A. Carpentier, L. Michel, S. Grihon, and J. J. Barrau. Buckling optimization of composite panels via lay-up tables. In 3rd European Conference on Computational Mechanics Solids, Structures and Coupled Problems in Engineering, Lisbon, Portugal, June 2006.

[9] F. X. Irisarri, A. Lasseigne, F. H. Leroy, and R. Le Riche. Optimal design of laminated composite structures with ply drops using stacking sequence tables. Composite Structures, 107:559-569, 2014.

[10] F. X. Irisarri, D. M. J. Peeters, and M. M. Abdalla. Optimisation of ply drop order in variable stiffness laminates. Composite Structures, 152:791-799, 2016.

[11] Y. M. Meddaikar, F. X. Irisarri, and M. M. Abdalla. Laminate optimization of blended composite structures using a modified shepard's method and stacking sequence tables. Structural and Multidisciplinary Optimization, 55(2):535-546, 2017.

[12] F. Farzan Nasab, H. J. M. Geijselaers, and A. de Boer. A multi-level set gradient based algorithm for buckling optimization of blended composite structures. 5th Aircraft Structural Design Conference. Royal Aeronautical Society, Manchester, 2016. 
[13] J. A. Bailie, R. P. Ley, and A. Pasricha. A summary and review of composite laminate design guidelines. Technical report NASA, NAS1-19347. Northrop Grumman-Military Aircraft Systems Division, 1997.

[14] Military Handbook - MIL-HDBK-17-3F: Composite Materials Handbook, Volume 3 - Polymer Matrix Composites Materials Usage, Design, and Analysis. U.S. Department of Defense, 2002.

[15] F. Farzan Nasab, H. J. M. Geijselaers, I. Baran, and A. de Boer. Generating the best stacking sequence table for the design of blended composite structures. In Advances in Structural and Multidisciplinary Optimization: Proceedings of the 12th World Congress of Structural and Multidisciplinary Optimization (WCSMO12), pages 779-788, 2018. 


\section{Paper D}

Optimization of the interacting stiffened skins and ribs made of composite materials

This chapter is based on the paper 'Optimization of the interacting stiffened skins and ribs made of composite materials', submitted to 'AIAA Journal' (2018). 



\title{
Optimization of the interacting stiffened skins and ribs made of composite materials
}

\author{
F. Farzan Nasab ${ }^{1)}$, H.J.M. Geijselaers ${ }^{1)}$, I. Baran ${ }^{1)}$, R. Akkerman ${ }^{1)}$, A. \\ de Boer ${ }^{1)}$
}

Submitted to AIAA Journal

${ }^{1)}$ Applied Mechanics, Engineering Technology, University of Twente, Enschede, The Netherlands

\begin{abstract}
A decomposition strategy for the structural optimization of a fiber reinforced aircraft wing box is proposed. The proposed method decomposes the wing box optimization into two levels: a system level and a subsystem level optimization. The ribs are the subsystems of the problem. Each rib has a local set of design variables and constraints. The loads on the ribs are the crushing loads caused by the bending of the wing. At the system level, the wing box skins are optimized while accounting for the effect of the skin design on the loads applied to the ribs. The sensitivity of the rib mass to the applied loads is evaluated using the Lagrange multipliers of the optimized rib design. To enhance the numerical efficiency of the two level optimization, the changes of the loads on the ribs are subjected to a reduction by Principal Component Analysis (PCA). In both the wing-level and the rib-level optimization problem, the level-set strategy for the optimization of composite structures, previously introduced by the authors, is employed. This method permits an advantageous use of coarse and fine finite element models employing a standard commercial finite element code. The proposed method is applied to the design of a composite horizontal tail plane. The accuracy of and the computational time savings by the proposed PCA-based reduction scheme are quantified.
\end{abstract}




\section{Introduction}

The structural optimization of an aircraft wing consisting of laminated fiber reinforced polymer composite skins, ribs, spars, and stringers is a highly complex problem. This is due to the large number of variables as well as structural and aerodynamic constraints influencing the design of panels and stiffeners. In the following, the generic approaches for solving a high dimensional engineering optimization problem such as an aircraft wing are discussed. Then the goal of developing the present research is described.

\subsection{Strategies for solving the optimization of complex systems}

Strategies aiming at solving a complex optimization problem can be divided into two major categories: monolithic architectures and distributed architectures [1]. Monolithic approaches aim at solving the optimization problem all-at-once [1, 2]. This means that all design variables and constraints are implemented in a single optimization problem. Formulating such an optimization problem is straightforward. However, treating a large number of design variables and constraints together with a complex (wing) model, severely affects the efficiency of the optimization method [3].

The distributed approaches aim at decomposing the entire optimization problem into several smaller subproblems (subsystems) while preserving the couplings among these subproblems [4]. In distributed approaches, the subsystems can be optimized in parallel to further improve the efficiency of the entire optimization method.

A large amount of research has been dedicated to the optimization of large-scale systems composed of interacting subsystems. See, for example, the Schmit and Ramanathan's [5] and Schmit and Mehrinfar's [6] multilevel structural decomposition approach, or Sobieski's concurrent subspace optimization approach [7]. The application of collaborative optimization to an aircraft design problem is studied in Sobieski and Kroo [8] and Braun et al. [9]. Collaborative optimization allows the subsystems to be optimized in parallel [3]. However, the strategies focusing on decomposing a problem into several interacting subsystems may fail to converge to the optimum of the original problem $[4,10,11]$. These strategies require a more complex algorithm to account for the interacting subsystems.

The global/local framework based on a general decomposition method (developed by Haftka and Watson $[12,13]$ ) has been applied to the optimization of a wing by Liu et al [3]. The optimization is divided into the global wing-level optimization and a local panel optimization. On the global level, constraints such as stress, displacement, flutter speed and buckling were applied. The spars and ribs are parametrized on the global level. On the local level, the panels between spars and ribs are optimized for buckling performance. A drawback for this method is that, on the local level, the optimization of the panels cannot be performed separately. The design of each panel influences the design of the rest of the panels via the load redistribution.

A type of decomposition technique called quasi-separable [12], breaks the large-scale problem into a system level and several subsystem optimizations where the subsystems 
are independent from each other. In this approach, the design of a subsystem is described by the local design variables and the global system variables, but no variable from the other subsystems [3]. This approach plays a major role in the optimization of large-scale engineering problems. Haftka and Watson [13] demonstrated that the quasi-separable decomposition technique can allow for the global optimization where most of the optimization procedure is performed on lower dimensional subsystems.

\subsection{Motivation and aim of the present research}

The computational cost of an optimization problem increases with the number of design variables [4]. The large number of design variables related to the optimization of a composite structure will exponentially add to the complexity and cost of a high dimensional wing optimization problem.

The ribs are subject to crushing loads (or Brazier loads) [14, 15] caused by the bending deformation of the wing [14]. The design of the skins affects the crushing load applied to the ribs by changing the bending stiffness of the wing. Moreover, the design of either the top or the bottom skin affects the design of the skin on the opposite side through changing the loads applied to the opposite skin. Consequently, the design of the primary parts of an aircraft wing, in particular skins and ribs, has to be looked at as a coupled problem.

Optimizing the interacting primary parts of an aircraft wing, in particular skins and ribs considering the crushing load, has attracted little attention in literature. This motivated the authors to investigate a methodology that is capable of efficiently optimizing the primary parts of an aircraft wing (here, skins and ribs) where fiber reinforced composite materials are used.

In the present research, a quasi-separable decomposition for the optimization of interacting skins and ribs of an aircraft wing box is proposed. At the system level (here called wing-level), the wing box skins are optimized while the effect of the skins design on the loads applied to the ribs is taken into account. The weight minimization of the skins is the goal of the wing-level optimization. The ribs are the subsystems (here called rib-level) of the problem. Each rib has a local set of design variables and constraints. The load on the ribs is influenced by the skin design variables, but is assumed not to be influenced by the design variables from other ribs (other subsystems). This allows to optimize the ribs in parallel. The skin-level and the rib-level optimization are coupled through the loads on the ribs. A coupling sensitivity calculation is thus required to account for this. To enhance the numerical efficiency of the proposed method, first, the use of Lagrange multipliers is suggested in the procedure of obtaining the sensitivity of the optimized weight of the ribs to skin design variables [16]. Secondly, a scheme based on Principal Component Analysis (PCA) is introduced and applied when computing the coupling sensitivities. The applicability of the proposed decomposition method is examined by application to a wing box optimization problem.

The majority of the research in the field of optimization of composite structures is fully or partially dependent on evolutionary algorithms, in particular a GA [17]. The 
reason is that a GA is a search technique well suited for optimization problems where discrete design variables are involved $([18,19])$ and the gradient of the objective or constraint function cannot be calculated. However, a GA is in general more time consuming than gradient-based algorithms [20]. In practice, often a finite element analysis is required to evaluate the objective and constraints of the optimization problem which makes the function evaluation computationally heavy. This makes a GA impractical for application to a real problem as it requires a large number of function evaluations. Gradient-based algorithms are required to have an efficient and fast optimization of complex structures. A fully gradient-based method has been proposed by the authors [17]. This method aims at optimizing the stacking sequences and thickness distribution of large-scale composite structures considering the design rules and manufacturing guidelines. In [21] it is shown that this proposed method can be successfully applied to the optimization problem of the skins of an aircraft wing where multiple load cases and constraints (such as buckling and strain) are applied. In the present research, both the wing-level and the rib-level optimization problem employ the level-set strategy for the optimization of composite structures, previously introduced in [17].

The rest of this paper is organized as follows: in Section 2, the method for the optimization of the stacking sequences and the thickness distribution is summarized. Section 3 discusses the quasi-separable method for the optimization of the skins and ribs as a coupled problem. In Section 4, the PCA model reduction scheme for the coupling sensitivity calculation is discussed. In Section 5, the result and the discussion of implementing the proposed method to the optimization problem of a wing are provided. The paper is concluded in Section 6 .

\section{Stacking sequence and thickness optimization}

A method has been recently proposed by the authors for the optimization of variable stiffness composite structures [17]. The method separates the optimization of the stacking sequences from the optimization of the thickness distribution. The optimized stacking sequences are obtained with respect to the loads applied to the structure and are stored in a reference table called Stacking Sequence Table (SST) [22, 23]. The laminates in the design of the structure are only allowed to be selected from the SST. The stacking sequence of a thicker laminate in an SST is obtained by adding plies to a thinner laminate. This results in the continuity of plies in adjacent panels and guarantees the manufacturability of the final design. Every laminate in an SST is required to satisfy several rules suggested for the design of a composite structure. The following design rules are enforced $[22,24]$ :

- Ply orientation, the ply orientation is restricted to the set: $\left\{0^{\circ},+45^{\circ},-45^{\circ}\right.$, and $90^{\circ}$ \}.

- Symmetry, the laminate should be symmetric with respect to its center line.

- Covering plies, the outermost ply has to have the orientation of either $+45^{\circ}$ or $-45^{\circ}$. 
- Disorientation, the maximum difference in the orientation of each two adjacent plies is $45^{\circ}$.

- Percentage rule, at least $10 \%$ of the total number of plies in each laminate has to be assigned to each orientation from the set $\left\{0^{\circ},+45^{\circ},-45^{\circ}\right.$, and $\left.90^{\circ}\right\}$.

- Balance, the total number of plies with $+45^{\circ}$ orientation is equal to the total number of plies with $-45^{\circ}$ orientation in a laminate.

- Contiguity, not more than 4 successive plies with a same orientation are allowed to stack together.

When an SST is used, it is guaranteed that the design rules and the ply continuity are fulfilled without adding an extra constraint to the optimization problem. A sample SST is shown in Figure 8.

Next, an auxiliary level-set function (LSF) is introduced to be used with an SST to prescribe the optimized thickness distribution over the structure. The level-set function $(\Phi)$ is discretized by interpolation among a limited set of $N_{\phi}$ design nodes with locations $X_{\phi}$. The design nodes are distributed over the structure. The values $\Phi\left(X_{\phi}\right)$ are the design variables in the optimization problem. The value of $\Phi$ at any location $X$ is obtained by the interpolation

$$
\Phi(X)=\sum_{n=1}^{N_{\Phi}} \Gamma^{n}(X) \Phi^{n}
$$

where $\Gamma^{n}(X)$ are interpolation functions and $\Phi^{n}$ represents $\Phi\left(X_{\phi}^{n}\right)$. In the present study, bilinear interpolation functions on quadrilateral domains, spanning multiple finite elements are used.

In the proposed method, the level-set function is used to select a ply stack from the SST. The number of plies $N_{\text {plies }}$ of the laminate at any point $X$ in the design domain $\Omega$ is determined through the level-set function by:

$$
N_{\text {plies }}(X)= \begin{cases}\max L V_{i} \mid L V_{i} \leq \Phi(X), & \text { if } \Phi(X) \geq \min L V_{i}, L V_{i} \in \mathrm{LV} \\ 0, & \text { otherwise }\end{cases}
$$

where LV represents the set of all ply stack values in the SST. According to Equation (2) the highest ply stack value below the level-set function at point $X$ gives the number of plies of the laminate covering point $X$.

Each finite element node obtains a level-set function value based on interpolation from the design node values. Within a finite element, the interpolation of the value of the element nodes specifies the level-set function.

Using the method summarized above, the continuous change of a design variable changes the thickness distribution in the design. Thus a change in a design variable changes the element stiffness matrices. This is the basis for the sensitivity analysis to iteratively update the design using a gradient based algorithm.

Detailed information on the use of the level-set based thickness optimization can be found in [17]. 


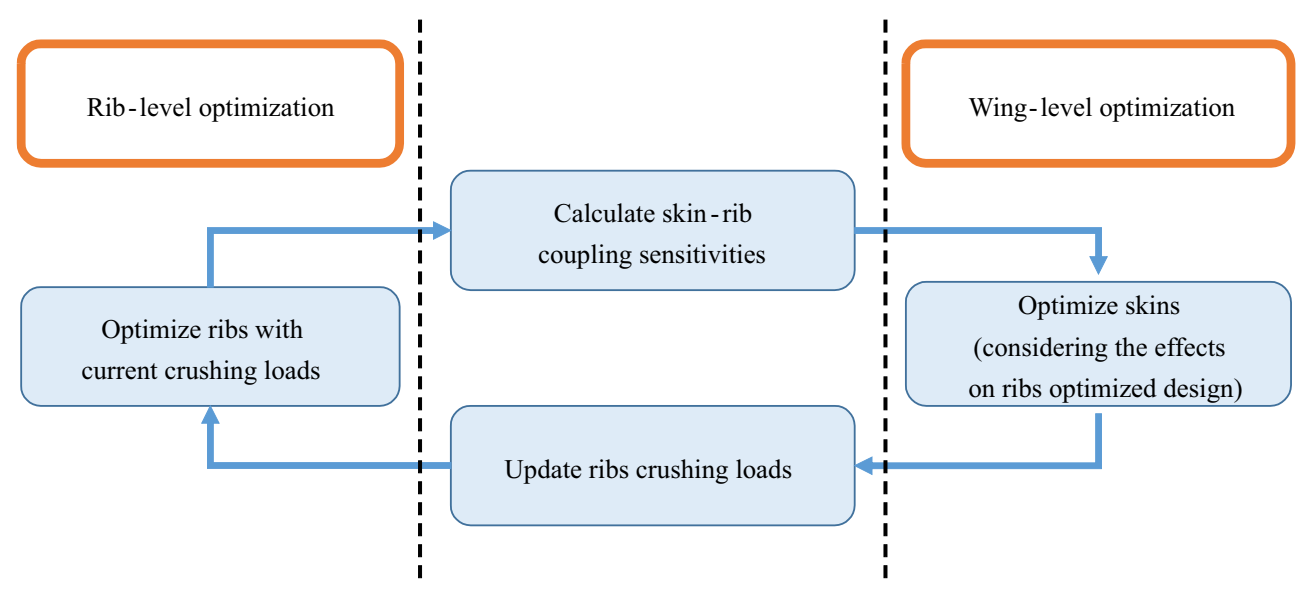

Figure 1: one full iteration of the proposed skin-rib optimization method.

\section{Quasi-separable decomposition strategy for the skin-rib coupled optimization}

Each full iteration of the optimization problem is divided into the skin-level and the rib-level optimization. The rib-level and the wing-level optimization are coupled via the crushing loads applied to the ribs from the skins. Figure 1 schematically shows one full iteration of the proposed skin-rib optimization method. In reality the rib design variables also affect the skins design through the load applied from the ribs to the skins. In the current research, however, this coupling is neglected. The crushing load applied to the ribs is caused by the wing box bending. The skin design affects the bending stiffness of the wing box and thus affects the crushing load applied to the ribs. The crushing load applied to the ribs is calculated with a nonlinear calculation and a coarse finite element model of the wing box. The optimization of top skin, bottom skin and ribs is done using separate fine finite element models. To each of these, the free body diagram loads obtained from the simulations by the coarse model are applied. In the following, the rib and the wing-level optimization as well as an efficient way to calculate the skin-rib coupling sensitivities are described in detail.

\subsection{Rib-level optimization}

The rib-level optimization is formulated as

$$
\begin{aligned}
\min & f_{\text {rib-level }}=W_{\text {rib }}\left(\Phi_{\text {rib }}^{N}\right) \\
\text { subject to } & g_{\text {rib }}^{i}\left(\Phi_{\text {rib }}^{N} ; \mathbf{L}_{\text {rib }}\right) \leq 0 \quad i=1 \text { to } m_{\text {rib }} \\
& \Phi_{\text {min }} \leq \Phi_{\text {rib }}^{n} \leq \Phi_{\max }
\end{aligned}
$$

where $W_{\text {rib }}$ represents the weight of the rib as a function of rib design variables vector $\Phi_{\mathrm{rib}}^{N} \cdot g_{\mathrm{rib}}^{i}$ represents the $i^{\text {th }}$ constraint applied to the optimization problem of the 
rib, $\mathbf{L}_{\text {rib }}$ represents the load vector applied to the rib, and $m_{\text {rib }}$ is the number of rib constraints. $\Phi_{\min }$ and $\Phi_{\max }$ denote the lower bound and the upper bound for the values at the design nodes, respectively.

In the rib-level optimization the load applied to each rib is kept constant. Since the rib load influences the constraints, the optimized design of a rib is a function of this load.

\subsection{Skin-rib coupling}

As mentioned earlier, the design of the skins influences the optimized design of the ribs through the crushing load. Therefore, after a rib-level optimization, the sensitivity of the optimized weight of a rib $\left(W_{\text {rib }}^{*}\right)$ to the skin design variables needs to be calculated.

As the optimized weight of a rib cannot be analytically defined as a function of skins design variables, this coupling sensitivity has to be obtained using a numerical derivative calculation scheme such as the forward difference method. Using the numerical forward difference scheme, for every perturbed skin design variable, the rib has to be optimized with the updated load vector so that the coupling sensitivities can be calculated. This requires a large number of computations. Here, the following chain rule is used:

$$
\frac{\partial W_{\text {rib }}^{*}}{\partial \Phi_{\text {skin }}^{N}}=\frac{\partial W_{\text {rib }}^{*}}{\partial \mathrm{g}_{\text {rib }}^{*}} \frac{\partial \mathrm{g}_{\text {rib }}^{*}}{\partial \Phi_{\text {skin }}^{N}}
$$

where $\Phi_{\text {skin }}^{N}$ is the vector of skins' design variables, and $g_{\text {rib }}^{*}$ represents the vector of rib active constraints at the optimized design. The first term in the right hand side of Equation (4) is the sensitivity of the optimized weight of the rib to active constraints of the rib. This term is calculated through [16]:

$$
\frac{\partial W_{\text {rib }}^{*}}{\partial \mathrm{g}_{\text {rib }}^{*}}=\boldsymbol{\mu}
$$

where $\boldsymbol{\mu}$ represents the vector of Lagrange multipliers of the rib active constraints at the optimized design. As the Lagrange multipliers of the rib active constraints are provided by the rib optimization routine, the term $\partial W_{\text {rib }}^{*} / \partial g_{\text {rib }}^{*}$ can be obtained cheaply.

The second term in the right hand side of Equation (4) can be obtained through:

$$
\frac{\partial \mathrm{g}_{\mathrm{rib}}^{*}}{\partial \Phi_{\mathrm{skin}}^{N}}=\frac{\partial \mathrm{g}_{\mathrm{rib}}^{*}}{\partial \mathbf{L}_{\mathrm{rib}}} \frac{\partial \mathbf{L}_{\mathrm{rib}}}{\partial \Phi_{\mathrm{skin}}^{N}}
$$

To calculate the term $\partial g_{\text {rib }}^{*} / \partial \mathbf{L}_{\text {rib }}$ in Equation (6), finite element analyses related to the rib active constraints have to be performed for every perturbed load vector applied to the rib. To calculate the term $\partial \mathbf{L}_{\text {rib }} / \partial \Phi_{\text {skin }}^{N}$, geometrically non-linear analyses equal to the number of skin design variables have to be performed.

In the Section 4, a model reduction scheme is introduced to calculate the coupling sensitivities of Equation (6) efficiently. 


\subsection{Wing-level optimization}

$\Phi_{\text {skin }}^{N}$ is composed of the top and bottom skin design variables vector, $\left(\Phi_{\text {Tskin }}^{N}\right)$ and $\left(\Phi_{\text {Bskin }}^{N}\right)$, respectively: $\Phi_{\text {skin }}^{N}=\left[\begin{array}{l}\Phi_{\text {Tskin }}^{N} \\ \Phi_{\text {Bskin }}^{N}\end{array}\right]$. The wing-level optimization is formulated
as:

$$
\begin{array}{lll}
\min & f_{\text {wing-level }}=W_{\text {Tskin }}\left(\Phi_{\text {Tskin }}^{N}\right)+W_{\text {Bskin }}\left(\Phi_{\text {Bskin }}^{N}\right)+\sum_{i=1}^{n_{\text {rib }}} W_{\text {rib }}^{*}\left(\mathbf{L}_{\text {rib }}\left(\Phi_{\text {skin }}^{N}\right)\right) \\
\text { s.t. } & g_{\text {Tskin }}^{i}\left(\Phi_{\text {Tskin }}^{N}, \mathbf{L}_{\text {Tskin }}\right) \leq 0 & i=1 \text { to } m_{\text {Tskin }} \\
& g_{\text {Bskin }}^{i}\left(\Phi_{\text {Bskin }}^{N}, \mathbf{L}_{\text {Bskin }}\right) \leq 0 & i=1 \text { to } m_{\text {Bskin }} \\
& \Phi_{\min } \leq \Phi_{\text {rib }}^{n} \leq \Phi_{\max }
\end{array}
$$

where $W_{\text {Tskin }}$, and $W_{\text {Bskin }}$ represent the weight of the top and the bottom skin, respectively. $W_{\mathrm{rib}^{i}}$ represents the weight of the $i^{\text {th }}$ rib. The top skin and the bottom skin constraints are represented by $g_{\text {Tskin }}$ and $g_{\text {Bskin }}$, respectively. $\mathbf{L}_{\text {Tskin }}$ and $\mathbf{L}_{\text {Bskin }}$ represent the load vector applied to the top and the bottom skin, respectively. The number of the top and the bottom skin constraints is denoted by $m_{\text {Tskin }}$, and $m_{\text {Bskin }}$, respectively.

The top and the bottom skin are also coupled through the loads. In the wing-level optimization, the effect of this coupling is implemented in the constraints of the optimization problem. Therefore, in Equation (7), the weight of either of the skins is only a function of its own design variables while the constraints of either of the skins are functions of both skins' design variables.

The sensitivity of the objective function in the wing-level is calculated through:

$$
\frac{\partial f_{\text {wing-level }}}{\partial \Phi_{\text {skin }}^{N}}=\frac{\partial W_{\text {Tskin }}}{\partial \Phi_{\text {Tskin }}^{N}}+\frac{\partial W_{\text {Bskin }}}{\partial \Phi_{\text {Bskin }}^{N}}+\sum_{i=1}^{n_{\text {rib }}} \frac{\partial W_{\text {rib }^{i}}^{*}}{\partial \Phi_{\text {skin }}^{N}}
$$

The last term in the right hand side of Equation $(8),\left(\partial W_{\text {rib }}^{*} / \partial \Phi_{\text {skin }}^{N}\right)$, is the skin-rib coupling term and is calculated as described in Section 3.2.

To calculate the term $\partial W_{\text {Tskin }} / \partial \Phi_{\text {Tskin }}^{N}$, a numerical forward difference scheme is used:

$$
\frac{\partial W_{\text {Tskin }}}{\partial \Phi_{\text {Tskin }}^{i}}=\frac{W_{\text {Tskin }}\left(\Phi_{\text {Tskin }}^{N}+\delta \Phi_{\text {Tskin }}^{i}\right)-W_{\text {Tskin }}\left(\Phi_{\text {Tskin }}^{N}\right)}{\delta \Phi_{\text {Tskin }}^{i}}
$$

The term $\partial W_{\text {Bskin }} / \partial \Phi_{\text {Bskin }}^{N}$ in Equation (8) is calculated in the same fashion as described in Equation (9).

Once the design of a skin has changed, the loads applied to both skins will change. Therefore, the top and the bottom skin designs are coupled through the loads. The 
constraint sensitivities in the wing-level optimization are defined as:

$$
\begin{gathered}
\frac{\partial g_{\text {Tskin }}}{\partial \Phi_{\text {skin }}^{N}}=\left[\begin{array}{l}
\partial g_{\text {Tskin }} / \partial \Phi_{\text {Tskin }}^{N} \\
\partial g_{\text {Tskin }} / \partial \Phi_{\text {Bskin }}^{N}
\end{array}\right] \\
\frac{\partial g_{\text {Bskin }}}{\partial \Phi_{\text {skin }}^{N}}=\left[\begin{array}{l}
\partial g_{\text {Bskin }} / \partial \Phi_{\text {Tskin }}^{N} \\
\partial g_{\text {Bskin }} / \partial \Phi_{\text {Bskin }}^{N}
\end{array}\right]
\end{gathered}
$$

As the $\partial g_{\text {Tskin }} / \partial \Phi_{\text {skin }}^{N}$ and $\partial g_{\text {Bskin }} / \partial \Phi_{\text {skin }}^{N}$ are obtained in the same fashion, here, we only describe the way $\partial g_{\text {Tskin }} / \partial \Phi_{\text {skin }}^{N}$ is calculated.

A top skin design variable affects the constraints on the top skin through both changing the load applied to the top skin and changing the top skin thickness distribution. Therefore, to calculate $\partial g_{\text {Tskin }} / \partial \Phi_{\text {Tskin }}^{N}$, using the numerical forward difference scheme, for every perturbed top skin design variable, the constraints have to be calculated with the updated loads and the updated thickness distribution.

To calculate $\partial g_{\text {Tskin }} / \partial \Phi_{\text {Bskin }}^{N}$, one can write:

$$
\frac{\partial g_{\text {Tskin }}}{\partial \Phi_{\text {Bskin }}^{N}}=\frac{\partial g_{\text {Tskin }}}{\partial \mathbf{L}_{\text {Tskin }}} \frac{\partial \mathbf{L}_{\text {Tskin }}}{\partial \Phi_{\text {Bskin }}^{N}}
$$

The two terms in the right hand side of Equation (11) are calculated in the same fashion as discussed for Equation (6).

Note that to calculate the coupling sensitivities (see Equations. (11), and (6)) in each full iteration of the optimization problem, the sensitivity of the load vector applied to the top skin, bottom skin, and each rib, to the skins' design variables is required. This, in total requires a number of geometric non-linear analyses equal to the number of skins' design variables in each full iteration of the optimization problem.

The flowchart of the proposed two-level method is shown in Figure 2.

\section{A model reduction scheme for coupling sensitivi- ties}

Calculation of the coupling sensitivities requires performing geometric nonlinear analysis (to obtain load derivatives) as well as a constraint related finite element analysis (such as buckling or compliance), equal to the number of skins' design variables. The analyses related to the coupling terms have to be performed in each full iteration (including rib-level and wing-level) of the optimization problem.

Here, a Principal Component Analysis (PCA) based model reduction scheme is introduced to calculate the coupling sensitivities in each full iteration of the optimization problem. The motivation is to reduce the number of finite element analyses required for calculating the coupling sensitivities. 


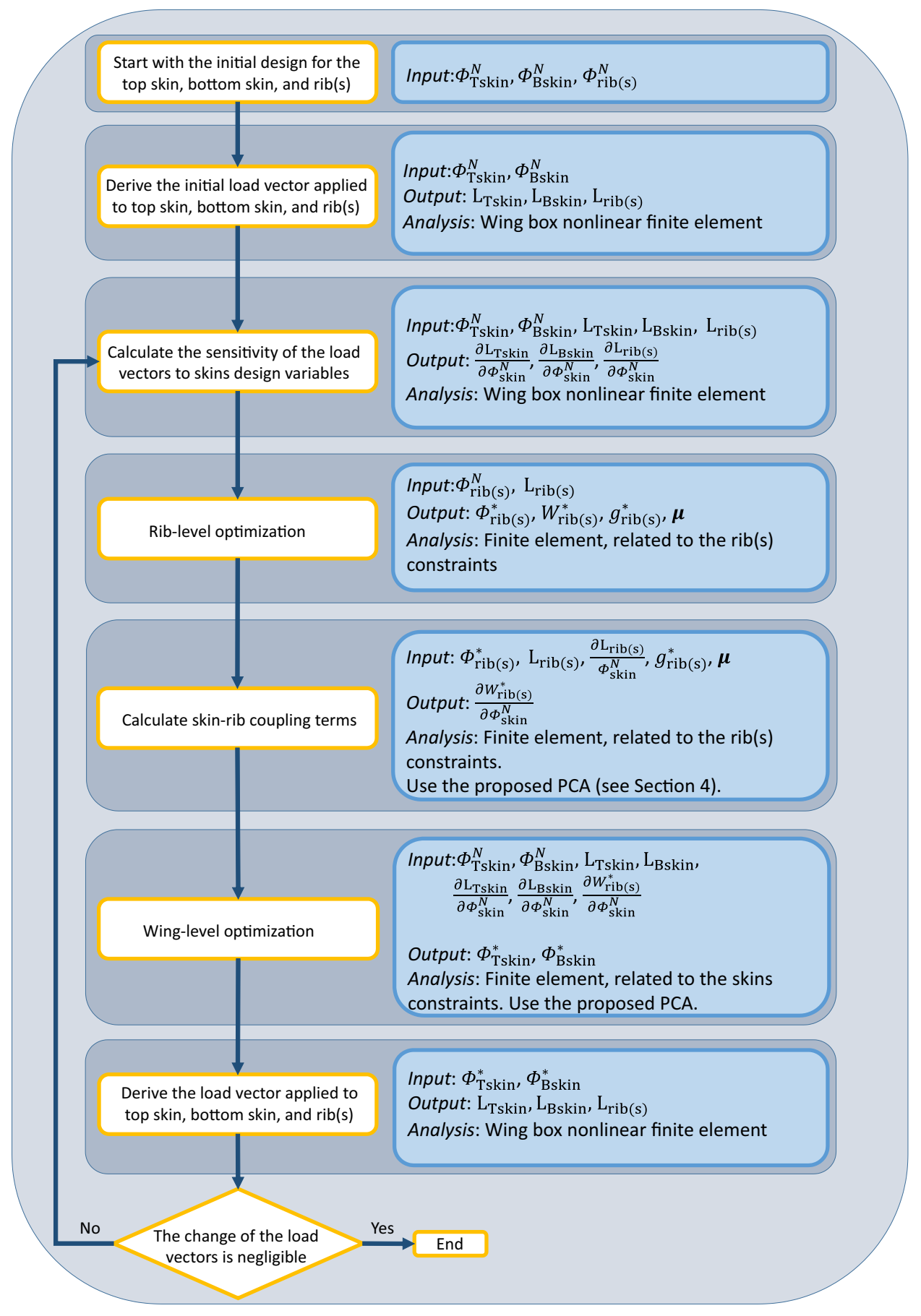

Figure 2: The flowchart of the proposed two-level skin-rib optimization method. $\Phi^{*}$ represents the vector of optimized design variables. 
We define the derivative of the (internal) load vector (applied to skins or ribs) to the skins' design variables by matrix $\mathbf{L}_{\Phi}$ :

$$
\mathbf{L}_{\Phi}=\frac{\partial \mathbf{L}}{\partial \Phi_{\text {skin }}^{N}}
$$

The PCA results in a subspace $\boldsymbol{\Psi}$ defined by orthonormal basis vectors $\psi_{i}$. The subspace $\boldsymbol{\Psi}$ for the matrix $\mathbf{L}_{\Phi}$ can be obtained by performing an eigenvalue decomposition of the correlation matrix $\mathbf{C}$ :

$$
\mathbf{C}=\mathbf{L}_{\Phi}^{T} \mathbf{L}_{\Phi}
$$

For the square matrix $\mathbf{C}$ the eigenvector matrix $\mathbf{V}$ can be calculated such that:

$$
\mathrm{CV}=\Lambda \mathrm{V}
$$

where $\mathbf{V}$ is the matrix of normalized eigenvectors and $\boldsymbol{\Lambda}$ is a diagonal matrix storing eigenvalues of matrix $\mathbf{C}$. The eigenvector matrix $\mathbf{V}$ is an orthonormal matrix: $\mathbf{V} \mathbf{V}^{T}=$ I.

The eigenvectors of matrix $\mathbf{C}$ in Equation (13) define the subspace $\boldsymbol{\Psi}$ for the load derivative matrix $\mathbf{L}_{\Phi}$. The following expression maps the load derivative matrix from the space defined by design variables $(\Phi)$ to the subspace $\boldsymbol{\Psi}$ defined by the eigenvectors V obtained from Equation (14):

$$
\mathbf{L}_{\Psi}=\mathbf{L}_{\Phi} \mathbf{V}
$$

The matrix $\mathbf{L}_{\Phi}$ can be defined in terms of the matrix $\mathbf{L}_{\Psi}$ through post multiplying Equation (15) by $\mathbf{V}^{T}$ :

$$
\mathbf{L}_{\Phi}=\mathbf{L}_{\Psi} \mathbf{V}^{T}
$$

By sorting eigenvectors based on the descending eigenvalues and removing the eigenvectors with the lowest eigenvalues, $\mathbf{L}_{\Phi}$ is approximated by:

$$
\mathbf{L}_{\Phi} \approx \mathbf{L}_{\widetilde{\mathbf{\Psi}}} \widetilde{\mathbf{V}}^{T}
$$

where $\mathbf{L}_{\widetilde{\mathbf{\Psi}}}$ and $\widetilde{\mathbf{V}}$ represent the load derivative in the reduced subspace and the reduced eigenvector matrix, respectively.

Consider the skin-rib coupling sensitivity $\partial \mathrm{g}_{\text {rib }}^{*} / \partial \Phi_{\text {skin }}^{N}$ defined in Equation (6). With the definition given in Equation (12), Equation (6) can be rewritten as:

$$
\frac{\partial \mathrm{g}_{\mathrm{rib}}^{*}}{\partial \Phi_{\mathrm{skin}}^{N}}=\frac{\partial \mathrm{g}_{\mathrm{rib}}^{*}}{\partial \mathbf{L}_{\mathrm{rib}}} \mathbf{L}_{\Phi}^{\mathrm{rib}}
$$

where $\mathbf{L}_{\Phi}^{\text {rib }}$ represents the derivative of the load vector applied to a rib with respect to skins' design variables. Substituting Equation (17) into Equation (18), gives:

$$
\frac{\partial \mathrm{g}_{\text {rib }}^{*}}{\partial \Phi_{\text {skin }}^{N}} \approx \frac{\partial \mathrm{g}_{\text {rib }}^{*}}{\partial \mathbf{L}_{\text {rib }}} \mathbf{L}_{\widetilde{\Psi}}^{\mathrm{rib}} \widetilde{\mathbf{V}}_{\text {rib }}^{T}
$$


We define the term $\partial g_{\text {rib }}^{*} / \partial \widetilde{\mathbf{\Psi}}$ as:

$$
\frac{\partial g_{\mathrm{rib}}^{*}}{\partial \widetilde{\Psi}}=\frac{\partial g_{\mathrm{rib}}^{*}}{\partial \mathbf{L}_{\mathrm{rib}}} \mathbf{L}_{\widetilde{\Psi}}^{\mathrm{rib}}
$$

where $\partial g_{\text {rib }}^{*} / \partial \widetilde{\Psi}$ is the derivative of the rib active constraints mapped into the reduced subspace $\widetilde{\boldsymbol{\Psi}}$. Substituting Equation (20) into Equation (19) gives:

$$
\frac{\partial \mathrm{g}_{\mathrm{rib}}^{*}}{\partial \Phi_{\mathrm{skin}}^{N}} \approx \frac{\partial g_{\mathrm{rib}}^{*}}{\partial \widetilde{\boldsymbol{\Psi}}} \widetilde{\mathbf{V}}_{\mathrm{rib}}^{T}
$$

To calculate the term $\partial g_{\text {rib }}^{*} / \partial \widetilde{\boldsymbol{\Psi}}$ one can write:

$$
\frac{\partial g_{\mathrm{rib}}^{*}}{\partial \widetilde{\psi}^{i}}=\frac{g_{\mathrm{rib}}^{*}\left(\mathbf{L}_{\mathrm{rib}}+\delta \cdot \mathbf{L}_{\widetilde{\mathbf{\Psi}}^{i}}^{\mathrm{rib}}\right)-g_{\mathrm{rib}}^{*}\left(\mathbf{L}_{\mathrm{rib}}\right)}{\delta} \quad i=1 \text { to } N_{\widetilde{\Psi}}
$$

where $\mathbf{L}_{\widetilde{\Psi}^{i}}^{\text {rib }}$ represents the $i^{\text {th }}$ column of the matrix $\mathbf{L}_{\widetilde{\Psi}}^{\text {rib }}$, and $N_{\widetilde{\Psi}}$ is the dimension of the reduced subspace $\widetilde{\boldsymbol{\Psi}}$.

Normally, calculating the term $\partial \mathrm{g}_{\text {rib }}^{*} / \partial \Phi_{\text {skin }}^{N}$ requires performing finite element analyses of the rib equal to the number of skin design variables. Using the proposed PCA scheme, the number of these finite element analyses is equal to the dimensions of the reduced subspace. As the number of dimensions of the reduced subspace is smaller than the number of skin design variables, fewer finite element analyses are required in calculating the coupling sensitivities using the proposed model reduction scheme. Moreover, the proposed method mathematically justifies the couplings that can be neglected.

Here, the proposed PCA is described for the derivatives of the skin-rib coupling terms. The same procedure is used to calculate the derivatives of the top skin-bottom skin coupling terms.

\section{Application, results, and discussion}

The proposed method is applied to the skin-rib optimization problem of a wing box. In this section, first the wing box finite element model is introduced. Then, the used SSTs and the definition of the optimization problem for this specific example are described. Finally, the results of the proposed decomposition are shown and discussed.

\subsection{Finite element model of the wing box}

The finite element model of the wing box is shown in Figure 3. The wing box consists of two skins, 13 ribs, a front and an aft spar, and 11 stringers in each skin.

The dimensions of the wing box are shown in Figure 4. 

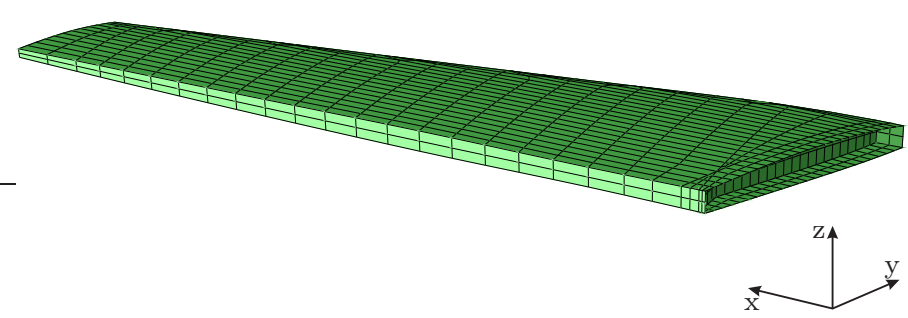

(a) Wing box finite element model.

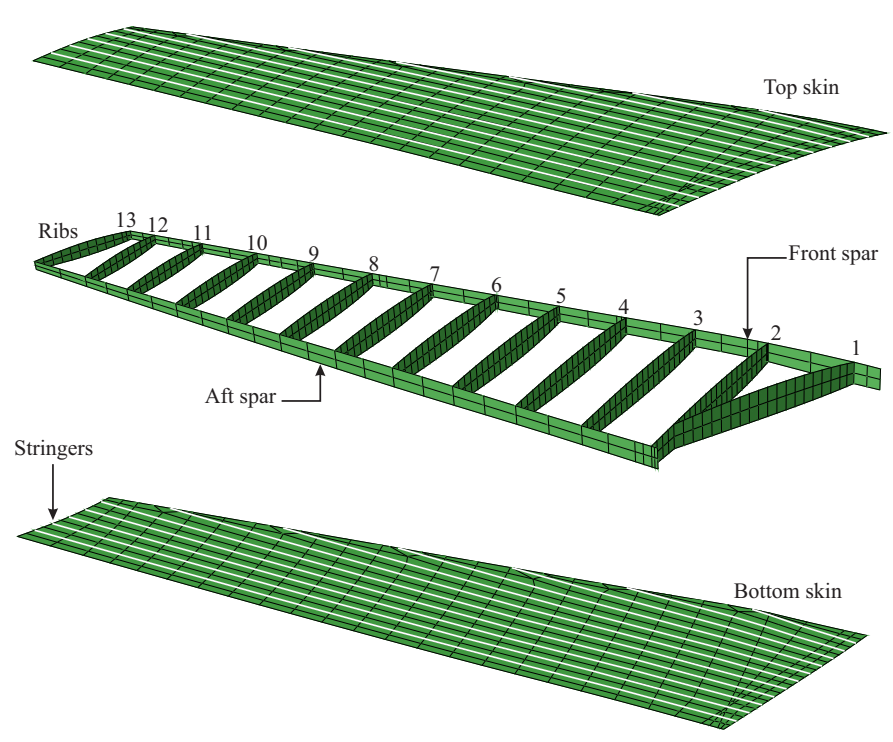

(b) The disassembled finite element model of the wing box. The rib numbering is shown next to each rib.

Figure 3: The coarse finite element model of the wing box.

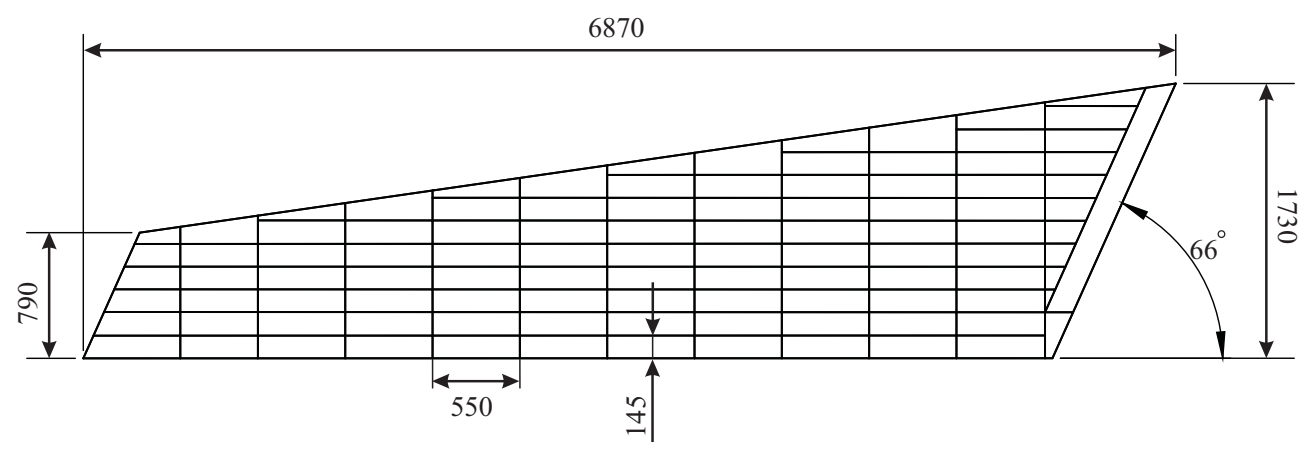

Figure 4: The dimensions of the wing box. All dimensions are given in millimeters. 


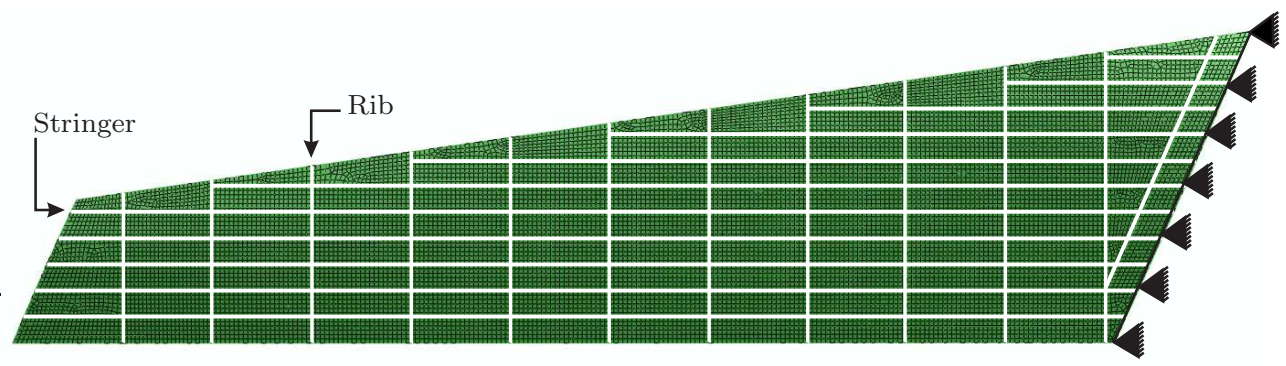

Figure 5: The fine model of the top skin. The skin is simply supported on the right edge and the out of plane translational degree of freedom for all edges and stiffeners is set to be zero.

The finite element model shown in Figure 3 is discretized by relatively coarse elements. As mentioned earlier, the loads applied to the ribs result from bending of the wing box. As the magnitude of wing box bending is determined by its bending stiffness, relatively coarse elements can be used to derive the rib loads. The level-set function can prescribe the same thickness distribution on both a coarse and a fine finite element model [17]. To lower the computation cost, the coarse model of the wing box is used to derive the rib loads and their derivatives and a fine model is used to analyze the constraints such as buckling and strain.

Here, three ribs are considered in the optimization. These are ribs 2, 7, and 11, as shown in Figure 3. The purpose of this choice was to evaluate the design of the rib at three different (span wise) locations of the wing box: close to the root, middle, and close to the tip.

The fine model of the top skin and the corresponding boundary conditions for the wing-level optimization are shown in Figure 5. A similar model and boundary conditions were used for the bottom skin in the wing-level optimization. As seen in Figure 5 , the stiffeners (ribs and stringers) divide the skin into multiple smaller panels. The skins are simply supported on the root edge and (since only the local buckling is considered) the out of plane translational degree of freedom for all edges and stiffeners is set to be zero. In both fine and coarse models the skins, ribs and spars are modeled with 4-node shell elements and the stringers are modeled with 2-node beam elements.

Figure 6 shows the fine model of the ribs and the corresponding boundary conditions used for the rib-level optimization. As seen in Figure 6, the ribs are simply supported and the out of plane translational degree of freedom of all boundary nodes is suppressed.

Table 1 provides detailed information about both the coarse and the fine models.

The wing box is subject to an upward bending load case as shown in Figure 7. This load remains constant during the optimization procedure. However, as discussed in Section 3 , the internal loads applied to the skins and ribs change as a result of changes in the skins design. 

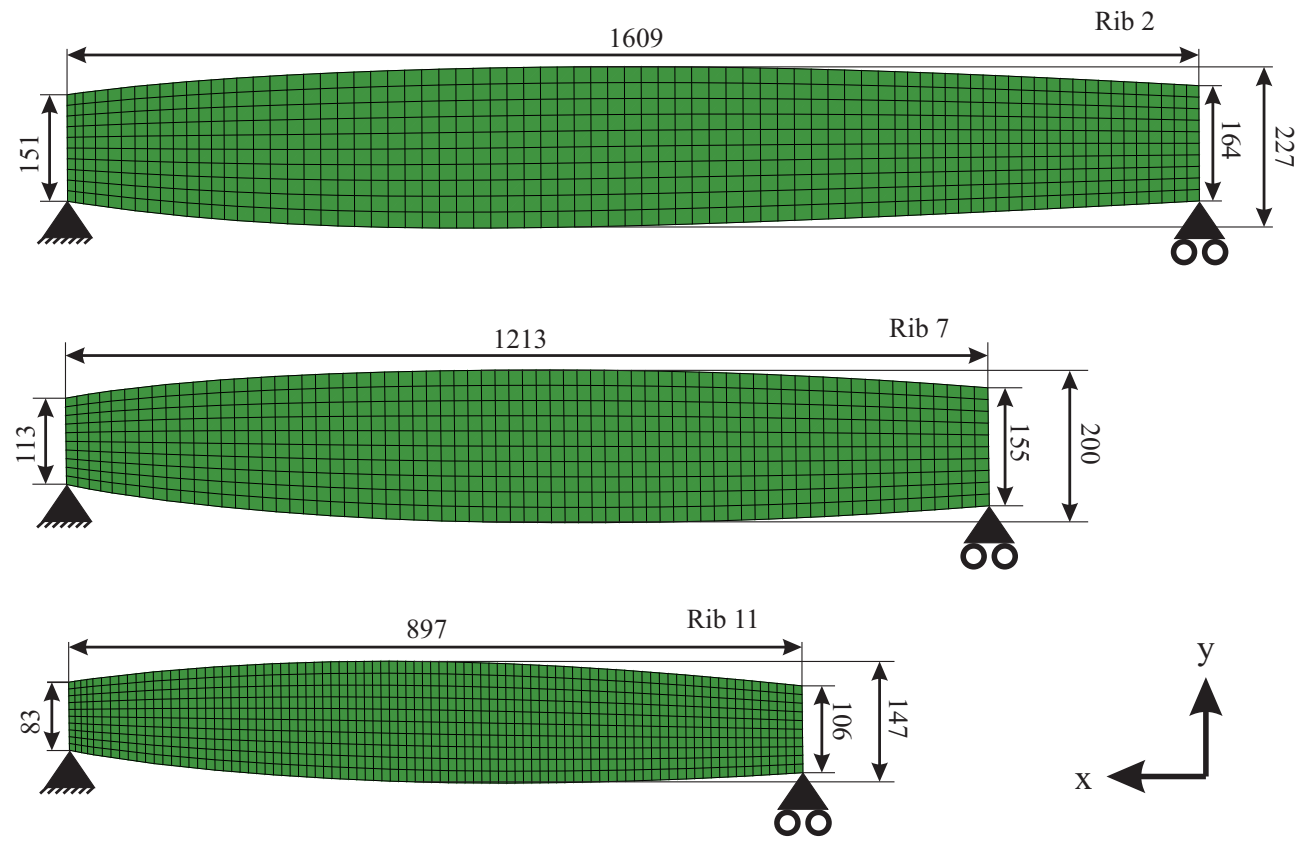

Figure 6: The fine model of ribs 2, 7, and 11 (see Figure 3 for rib numbering), used for optimizations. The ribs are simply supported and the dimensions are given in millimeter.

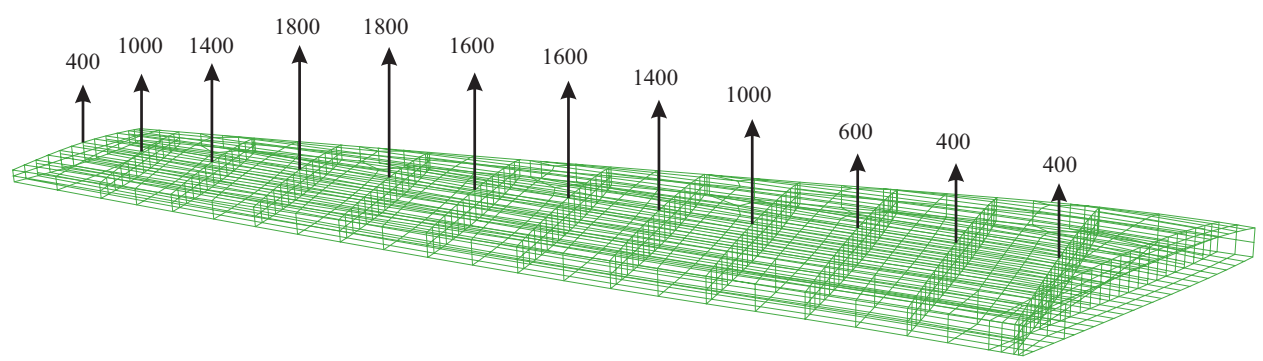

Figure 7: The upward bending load case applied to the wing box. The loads are given in $[\mathrm{N}]$. 
Table 1: Detailed information about the used finite element models. All dimensions are given in millimeters. DoF: Degree of Freedom.

\begin{tabular}{lccccc} 
& Wing box & Top skin/Bottom skin & Rib 2 & Rib 7 & Rib 11 \\
\hline Model type & Coarse & Fine & Fine & Fine & Fine \\
$\begin{array}{l}\text { 4-node shell element } \\
\text { (6 DOF per node) }\end{array}$ & 1572 & 13311 & 770 & 680 & 880 \\
$\begin{array}{l}2 \text {-node beam element } \\
\text { (3 DOF per node) }\end{array}$ & 358 & 3195 & - & - & - \\
$\begin{array}{l}\text { Typical element size } \\
\text { for shell elements }\end{array}$ & $\begin{array}{l}\text { skin: } 275 \times 72 \\
\text { spar: } 275 \times 77 \\
\text { ribm) } 113 \times 71\end{array}$ & $24 \times 24$ & $24 \times 24$ & $18 \times 18$ & $14 \times 10$ \\
$\begin{array}{l}\text { Typical element size } \\
\text { for beam elements } \\
(\text { mm) }\end{array}$ & stringer: 275 & & & & \\
\hline
\end{tabular}




\subsection{Used SSTs and optimization problem definition}

As described in Section 2, an optimized SST has to be generated with respect to the applied loads. This means that during the optimization procedure, the optimized SST related to each part has to be updated each time the loads are updated. This would result in an extremely expensive problem to be solved. To have an acceptable amount of computation, here, the optimization problem starts with an SST as shown in Figure 8. This SST satisfies the composite design rules mentioned in Section 2, but the stacking sequences are not optimized. When the entire skin-rib optimization problem using this SST has been solved, the loads applied to the top skin, bottom skin, and the ribs are extracted. Using these loads and the procedure developed by the authors [17], an SST with improved fiber orientations was generated for each part. As the loads applied to each rib differ, a unique SST for each rib is expected. Here, for the simplicity, the optimized SST generated for rib 2, is also used for ribs 7 and 11. During the optimization procedure, the SST for each part will remain constant. Figures 9, 10, and 11 show the optimized SST for the top skin, bottom skin, and ribs, respectively. The laminates in the optimized SST of the bottom skin have more $0^{\circ}$ fibers compared to those in the optimized SST of the top skin. For instance, a laminate with 40 plies from the optimized SST of the bottom skin, has 10 plies with $0^{\circ}$ fibers while the laminate with the same thickness from the optimized SST of the top skin has 6 plies with $0^{\circ}$ fibers. The reason is that the bottom skin (shown in Figure 3 ) is primarily subject to tensile loads along the $x$ direction. The bottom skin is subject to strain constraints in the optimization problem (this will be discussed later in this section). To improve the in-plane (extensional) stiffness of the laminates in the $x$ direction, plies with $0^{\circ}$ fiber orientation are required.

For the level-set based optimization, 9 design nodes are assigned to each skin and 21 design nodes are assigned to each rib. The number of design nodes assigned to the ribs is more than that assigned to the skins. The reason is that lightening holes are expected in the design of the ribs. Therefore, more design nodes are supplied to provide a more detailed design. Figure 12 shows the distribution of the design nodes on each part. As the distribution and numbering of the design variables follow the same fashion for all the studied ribs, only rib 2 is shown in Figure 12.

Here, the rib-level optimization is defined as:

$$
\begin{aligned}
\min & W_{\text {rib }} \\
\text { subject to } & \left(1-\lambda_{\text {rib }}^{i}\right) / \lambda_{\text {rib }}^{0} \leq 0 \quad i=1 \text { to } 50 \\
& C / C_{\text {all }}-1 \leq 0 \\
& \Phi_{\min } \leq \Phi^{n} \leq \Phi_{\max }
\end{aligned}
$$

where $\lambda_{\text {rib }}^{i}$ represents the $i^{\text {th }}$ buckling factor of the rib. The term $1-\lambda_{\text {rib }}^{i}$ is divided by $\lambda_{\text {rib }}^{0}$, which is defined as the initial buckling factor of the rib and is used to scale the buckling constraints to have an equal weight as the compliance constraint in the solution process. As seen in Equation (23), 50 buckling factors are considered as buckling constraints for each rib. $C$ and $C_{\text {all }}$ represent the compliance and an allowable for the rib compliance, respectively. The compliance constraint is imposed 


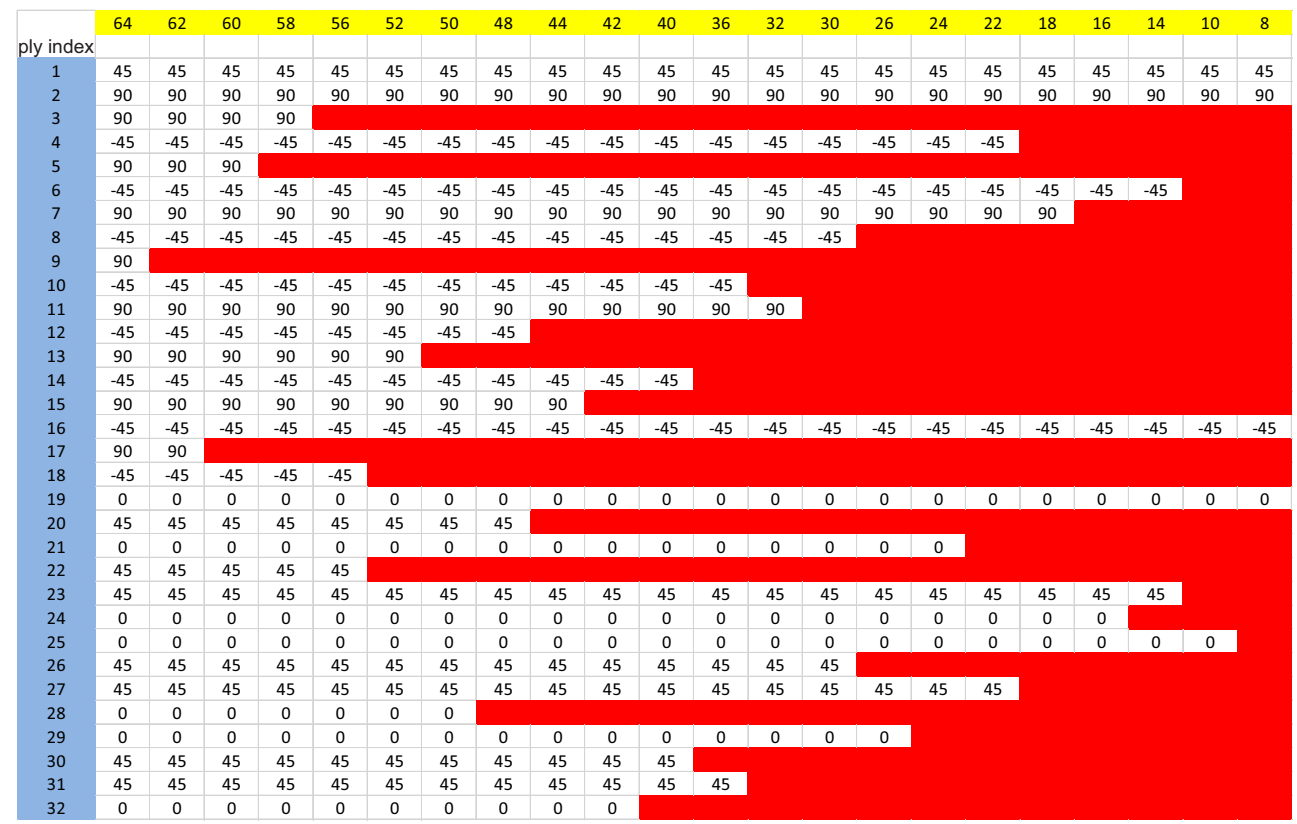

Figure 8: An SST with valid but not optimized stacking sequences. Fields marked red indicate dropped plies. Due to symmetry, only the stacking sequences of halflaminates are shown.

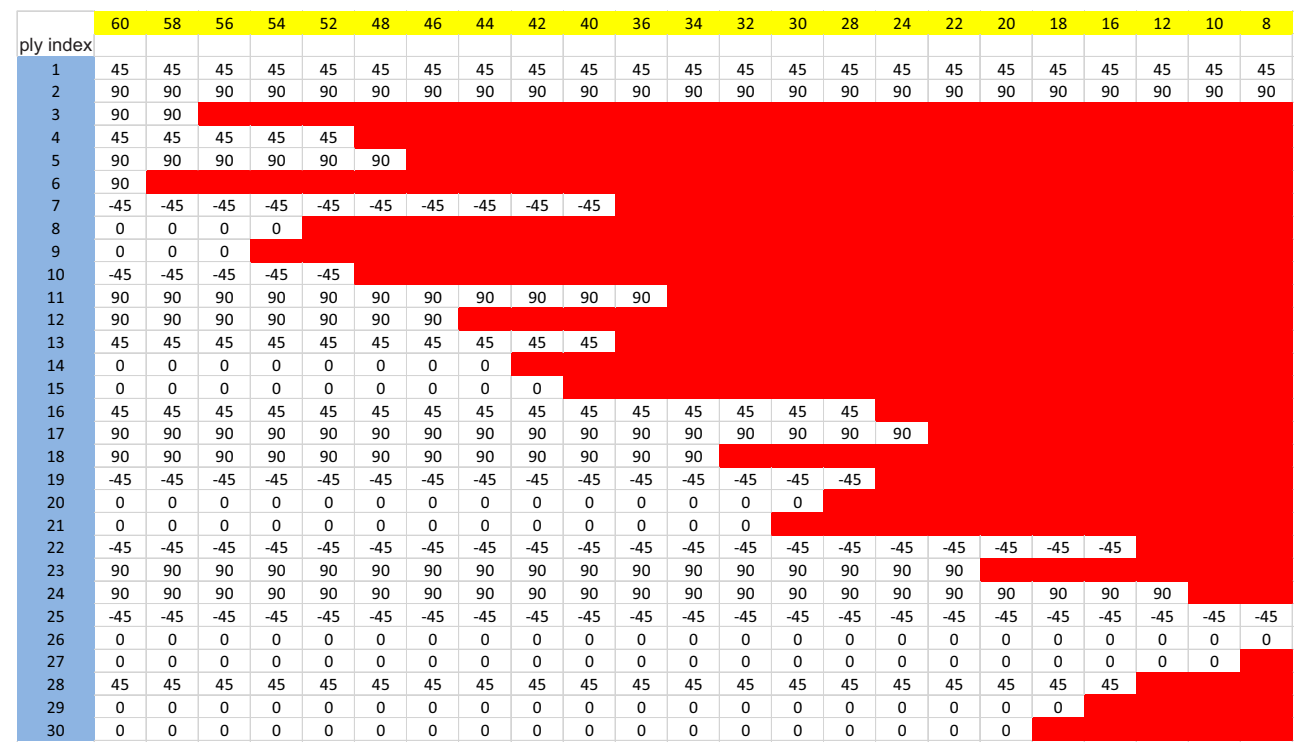

Figure 9: The optimized SST for the top skin. 


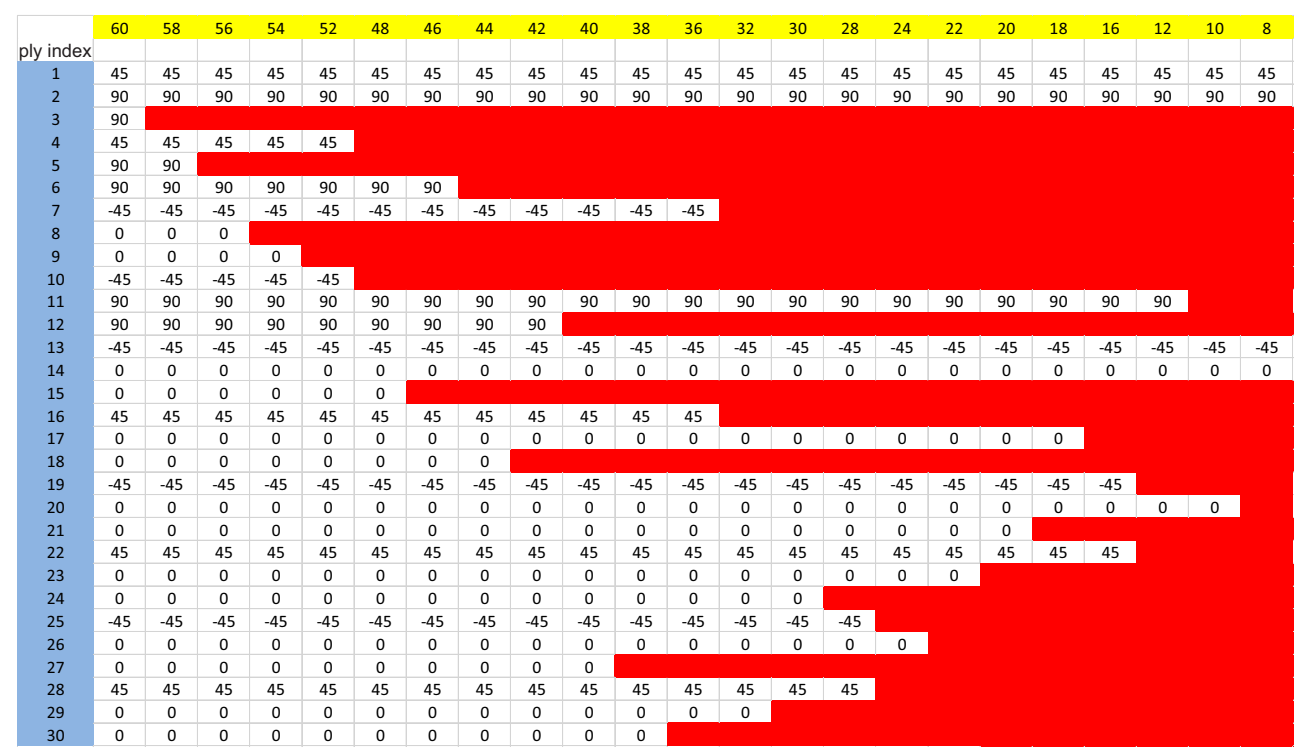

Figure 10: The optimized SST for the bottom skin.

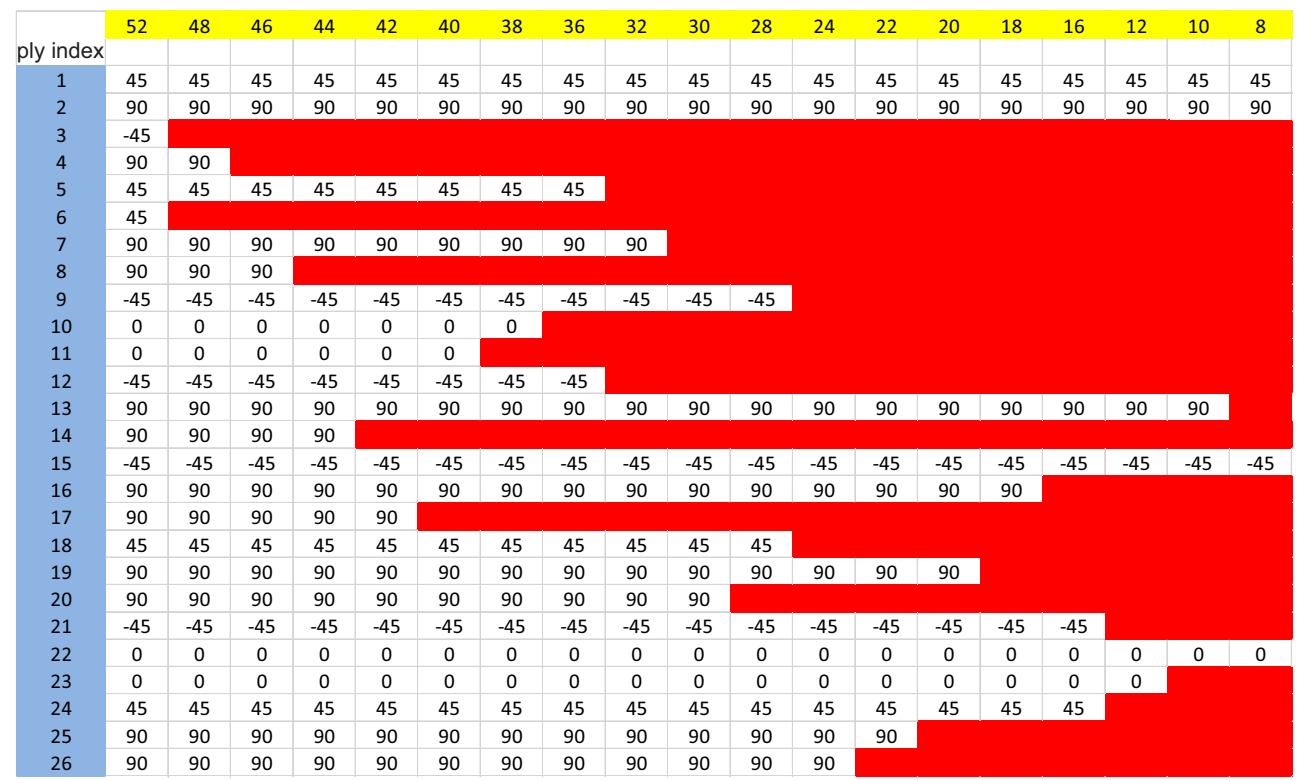

Figure 11: The optimized SST for the ribs. 


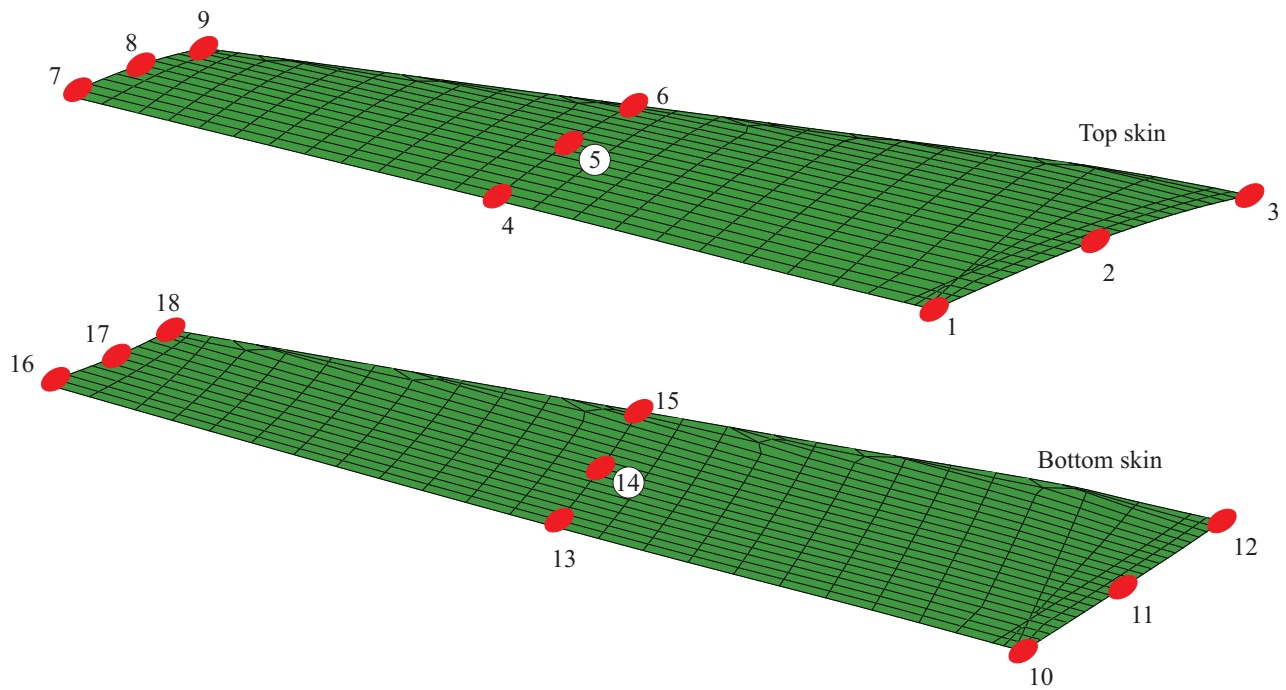

(a) Skins design variables and numbering.

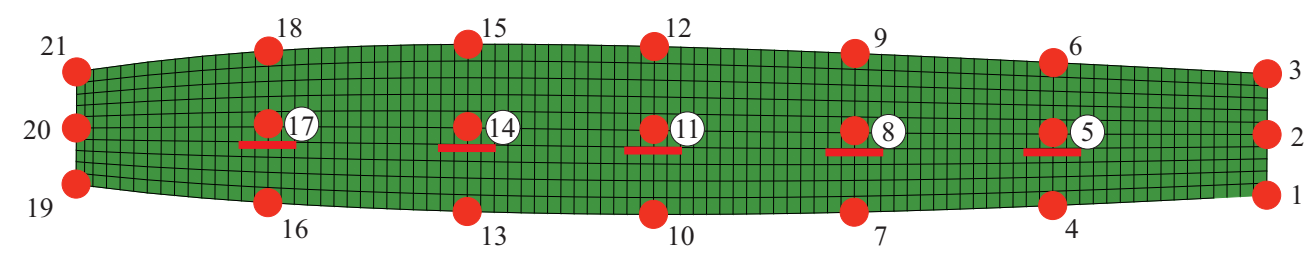

(b) Rib 2 design variables and numbering. The design variables that are allowed to fall below the first level to prescribe voids, are underlined.

Figure 12: The distribution and the numbering of design variables. 
Table 2: The allowable compliance value for each rib.

\begin{tabular}{cccc}
\hline & Rib 2 & Rib 7 & Rib 11 \\
Compliance (N.m) & 6 & 0.2 & 0.08 \\
\hline
\end{tabular}

to ensure that the ribs have a minimum required stiffness. The loads applied to the ribs located in different regions of the wing box are noticeably different. Correspondingly, an allowable for the compliance of each rib is defined as shown in Table 2:

$\Phi_{\min }$ is set to be 8 plies for the design nodes on the boundary of the ribs and zero for the rest of the design nodes. $\Phi_{\max }$ is set to be 52 plies (corresponding to the number of plies in the thickest laminate of the optimized SST for the ribs shown in Figure 11).

The wing-level optimization is defined as:

$$
\begin{array}{rlrl}
\min & W_{\text {Tskin }}+W_{\text {Bskin }}+\sum_{i=1}^{n_{\text {rib }}} W_{\text {rib }^{i}}^{*} & \\
\text { subject to } & \left(1-\lambda_{\text {Tskin }}^{i}\right) / \lambda_{\text {skin }}^{0} \leq 0 & & i=1 \text { to } 80 \\
& \left(\varepsilon_{\text {max_Bskin }}^{j} / \varepsilon_{\mathrm{T}}\right)-1 \leq 0 & j=1 \text { to } N_{\mathrm{P}} \\
& 1-\left(\varepsilon_{\text {min_Bskin }}^{j} / \varepsilon_{\mathrm{C}}\right) \leq 0 & j=1 \text { to } N_{\mathrm{P}} \\
& \Phi_{\min } \leq \Phi^{n} \leq \Phi_{\max } &
\end{array}
$$

where $\lambda_{\text {Tskin }}^{i}$ and $\lambda_{\text {skin }}^{0}$ represent the $i^{\text {th }}$ buckling factor and an initial buckling factor of the top skin, respectively. As seen in Equation (24), 80 buckling factors are considered as buckling constraints for the top skin. The bottom skin is subject to a strain constraint. An (central) element in each panel (skin panels are shown in Figure 5) of the bottom skin is evaluated for strain constraint. $\varepsilon_{\text {max_Bskin }}^{j}$ and $\varepsilon_{\text {min_Bskin }}^{j}$ represent the maximum and the minimum principal strains in the central element of the $j^{\text {th }}$ panel, respectively. $\varepsilon_{\mathrm{T}}$ and $\varepsilon_{\mathrm{C}}$ represent the allowable tensile and compressive strain, respectively. Here, $\varepsilon_{\mathrm{T}}=3.2 \mathrm{e}-4$ and $\varepsilon_{\mathrm{C}}=-1.8 \mathrm{e}-4$, were chosen. $N_{\mathrm{P}}$ is the number of panels which is equal to 99 . For the skins, $\Phi_{\min }$ and $\Phi_{\max }$ are set to be 8 and 60 plies, respectively.

\subsection{Results and discussion}

Figure 13 shows the contour plot of the thickness distribution for the top and the bottom skin at the optimum design. As seen in Figure 13, the front root of the top skin and the aft root of the bottom skin are covered with relatively thick laminates.

Figure 14 shows the contour plot of the thickness distribution for the ribs at the optimum point. As seen in Figure 14, an extended (narrow) void in the central region of each rib appears in the optimized design of each rib.

The evolution of the mass of the top and the bottom skin is shown in Figure 15. As seen in Figure 15, it takes 4 full iterations to reach a converged solution of the optimization problem. Figure 16 shows the progress of the mass of the ribs towards 


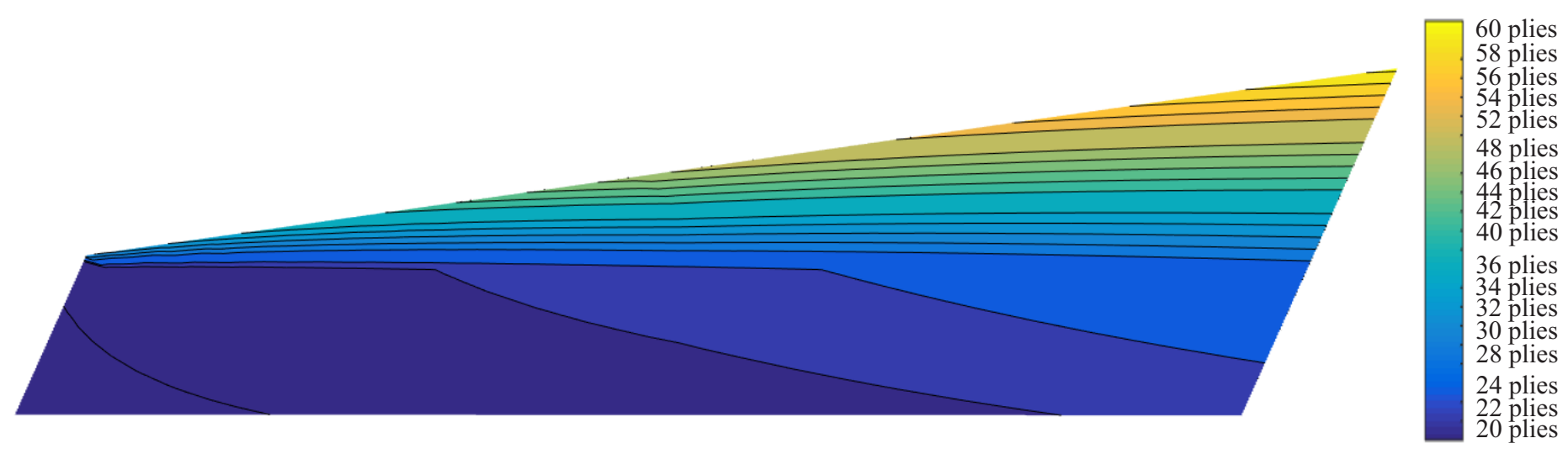

(a) The top skin thickness distribution.

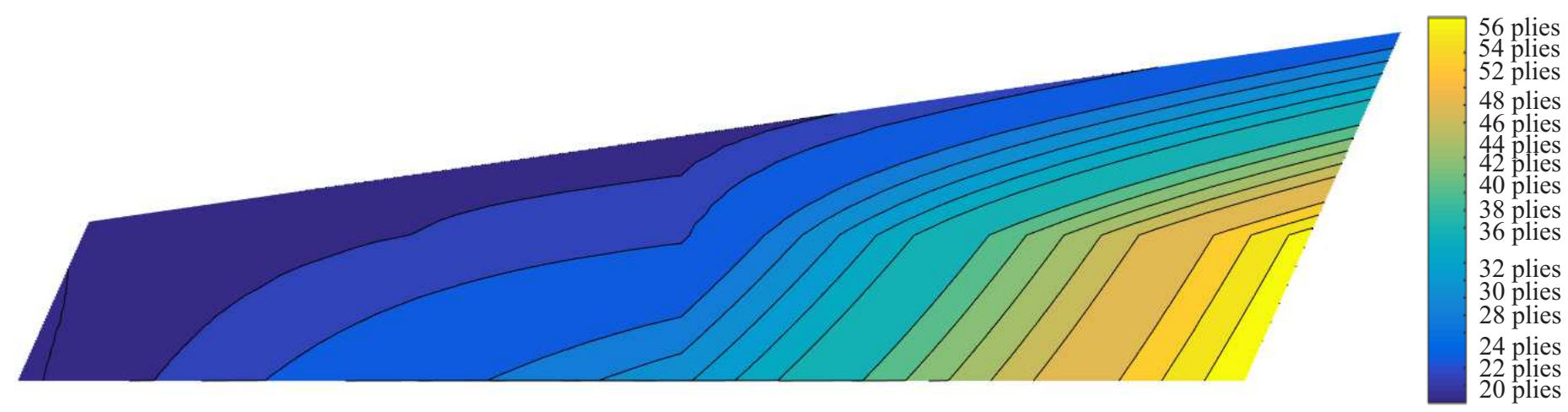

(b) The bottom skin thickness distribution

Figure 13: Contour plot of the thickness distribution of the top and the bottom skin at the point of optimum. 


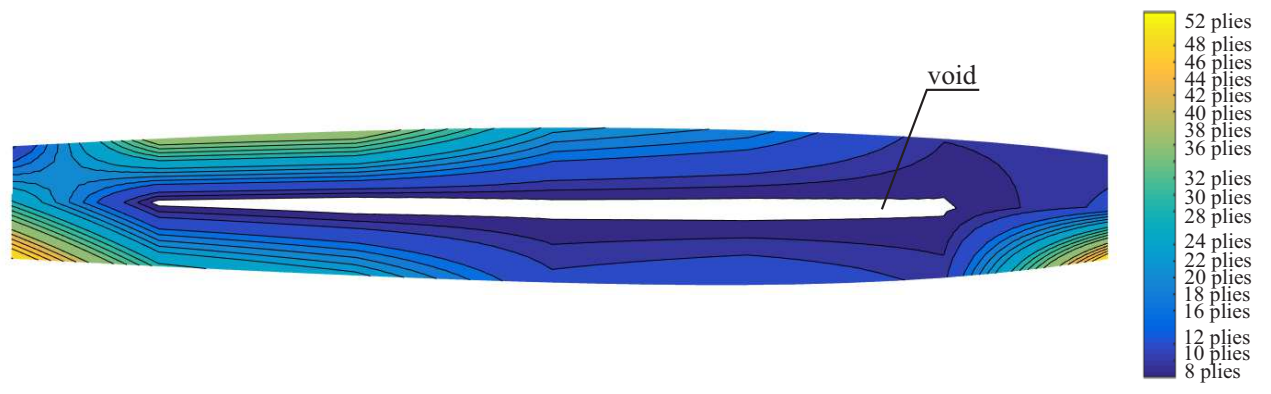

(a) Rib 2 thickness distribution.

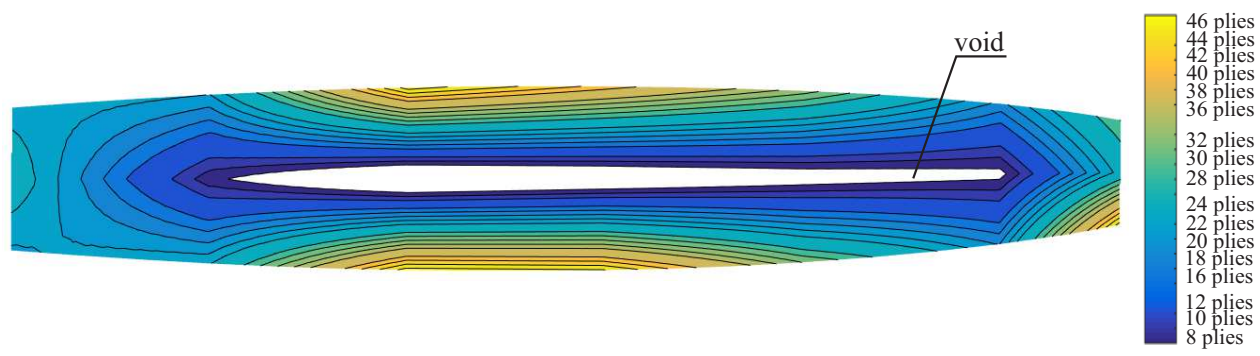

(b) Rib 7 thickness distribution.

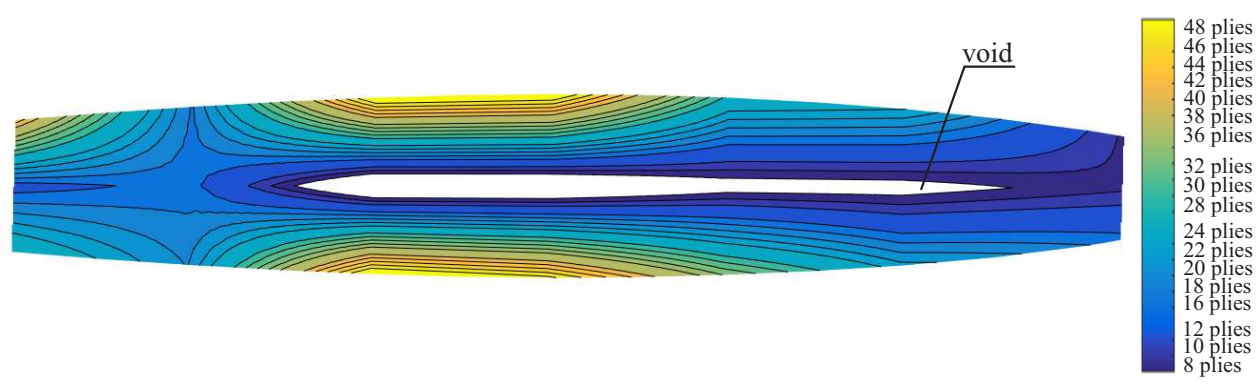

(c) Rib 11 thickness distribution.

Figure 14: Contour plot of the thickness distribution of the ribs at the point of optimum. 


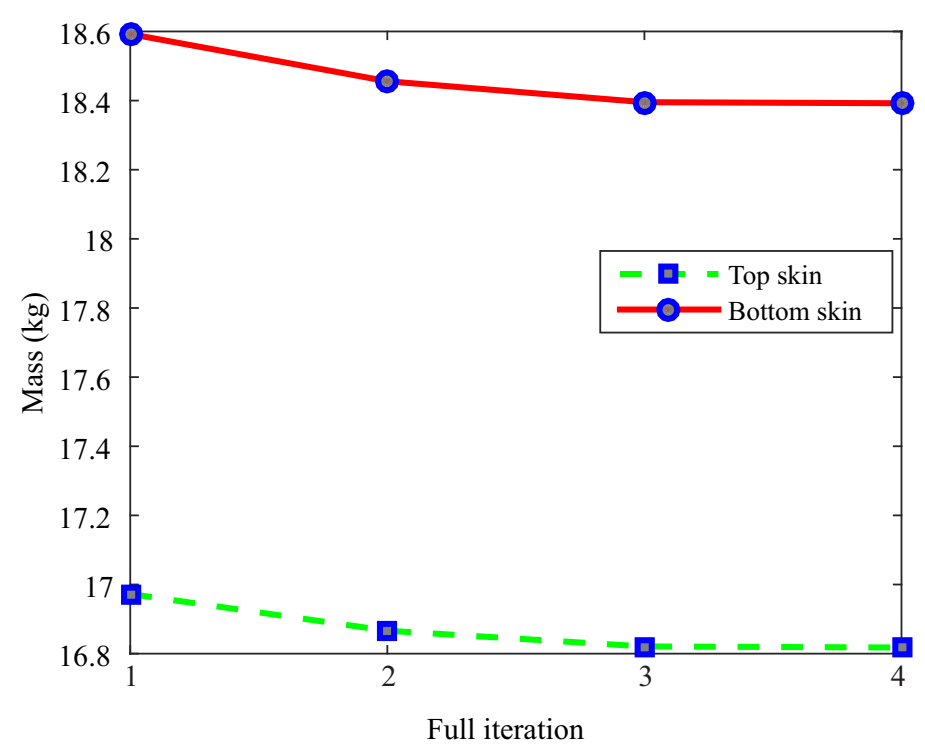

Figure 15: The evolution of the mass of the top and the bottom skin from the first full iteration of the optimization problem towards the optimized design. The mass of the top and the bottom skin initial design is $30.33 \mathrm{~kg}$.

optimum. As seen in Figure 16, the weight of the ribs increases from the first towards the fourth iteration. The reason is that the initial design of the skins is (considerably) thicker than the optimum design. This results in less wing box bending and thus smaller values of crushing loads applied to the ribs at the first iteration. The smaller values of the crushing loads allow a lower optimized weight for the ribs at the riblevel optimization of the first iteration. At the wing-level optimization of the first iteration, the weight of the skins decreases. This results in larger values of crushing loads applied to the ribs in the second iteration compared to the first iteration. Thus in the rib-level optimization of the second iteration, the weight of the ribs slightly increases.

Figure 17 shows the evolution of the weight of the wing box towards the optimum design. As seen in Figure 17, the weight of the wing box decreases regardless of the slight gradual increase of the ribs' weight. The reason is that the decrease in the skins weight dominates the slight increase of the rib weight.

Figure 18 shows the status of the top skin buckling constraints at the optimum point. The critical buckling constraint has the value of -0.67 where $\lambda_{\text {skin }}^{0}$ is 21.46. Figure 19 shows the status of the bottom skin strain constraints at the optimum point. As appears, the buckling constraint is not active while the the maximum principal strain is active at the optimum design.

Table 3 shows the status of the ribs' constraints at the optimum design. As seen in this table, the compliance constraint is the active constraint for the three studied ribs. Note that the small violation of the ribs' compliance constraint, is within the 


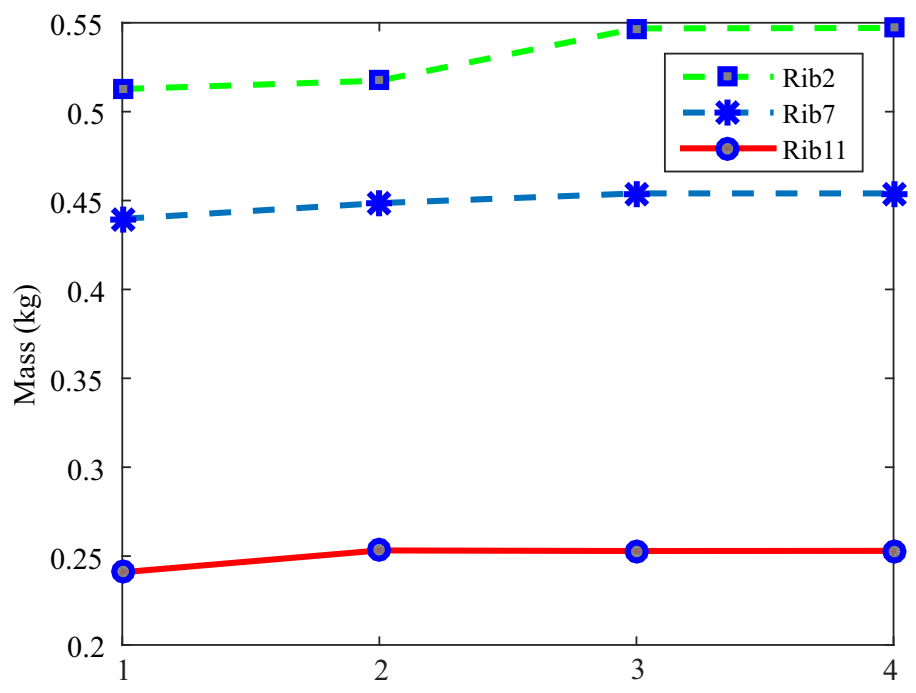

Full iteration

Figure 16: The evolution of the mass of ribs 2, 7, and 11 from the first full iteration of the optimization problem towards the optimized design.

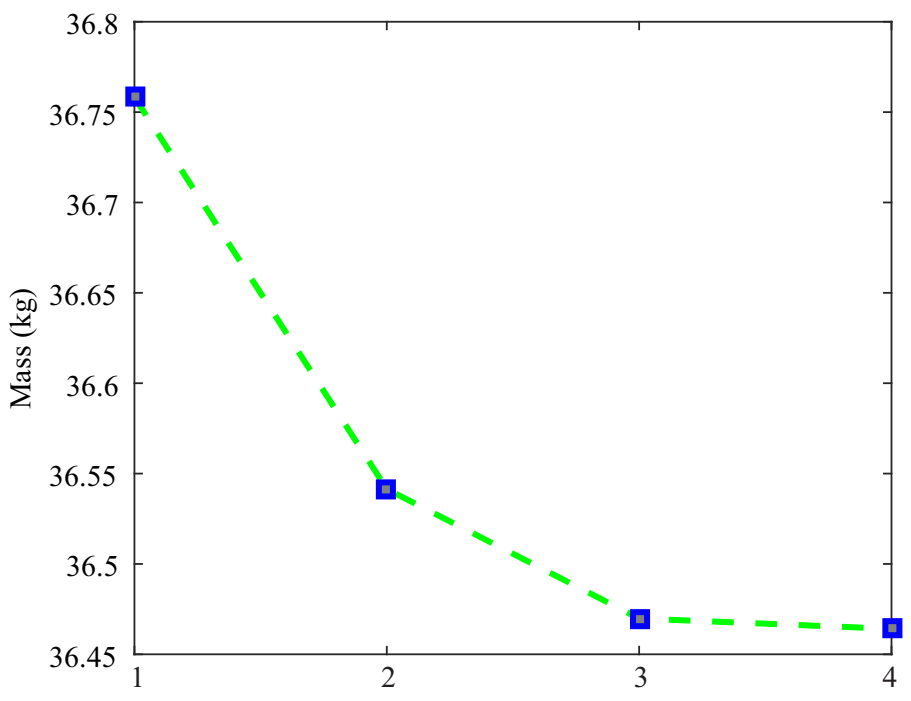

Full iteration

Figure 17: The evolution of the mass of the wing box (including the top skin, the bottom skin, and ribs 2, 7, and 11) from the first full iteration of the optimization problem towards the optimized design. The initial mass of the wing box is $63.26 \mathrm{~kg}$. 


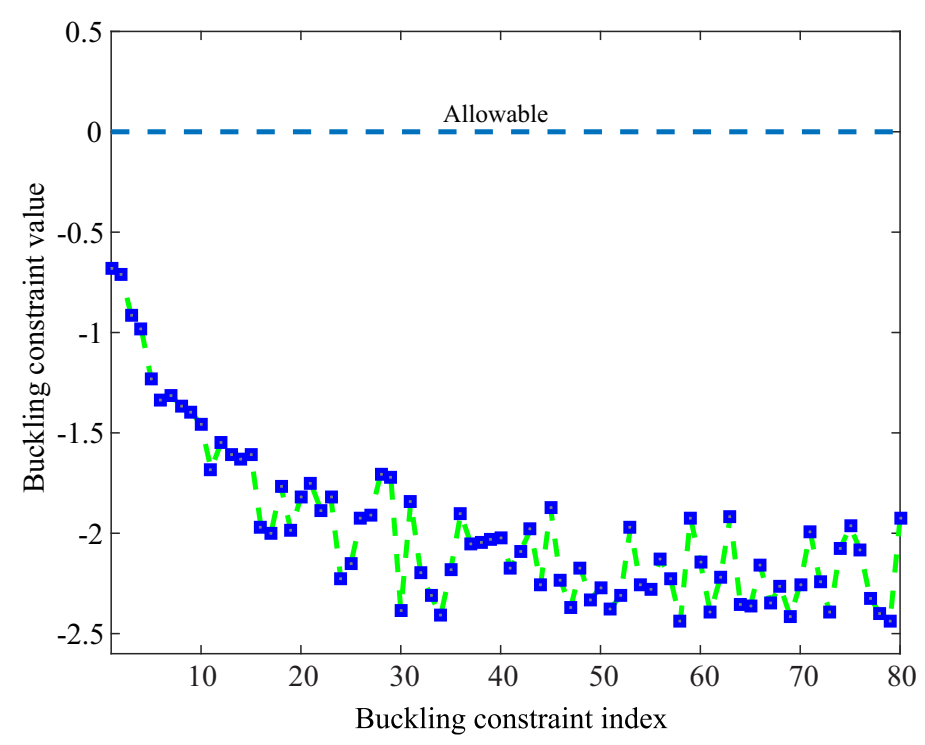

Figure 18: The value of the top skin buckling constraints $\left(\left(1-\lambda_{\text {Tskin }}^{i}\right) / \lambda_{\text {skin }}^{0}\right)$ where $\lambda_{\text {skin }}^{0}$ is 21.46. 80 buckling constraints are considered for the top skin.

Table 3: The status of the ribs' constraints at the optimum design.

\begin{tabular}{cccc}
\hline & Rib 2 & Rib 7 & Rib 11 \\
Critical buckling constraint & -0.07 & -0.16 & -0.18 \\
Compliance constraint & $1.3 \mathrm{e}-4$ & $6.2 \mathrm{e}-4$ & $2.1 \mathrm{e}-4$ \\
\hline
\end{tabular}

acceptable tolerance for constraint violation (the acceptable tolerance is 0.001 in the present study).

Table 4 provides detailed information on the number of iterations performed in the rib-level and wing-level optimization of each full iteration.

In each iteration of a rib-level optimization, a number of finite element analyses equal to the number of design variables on the rib $(+1)$ has to be performed. These finite element analyses are related to both buckling and compliance constraints. In each iteration of a wing-level optimization, the number of required buckling analyses is equal to the number of top skin design variables $(+1)$ and the number of strain analyses is equal to the number of bottom skin design variables $(+1)$. To calculate the coupling sensitivities in each full iteration, a number of nonlinear finite element analyses related to the wing box bending equal to the number of skins design variables is required. The average computation time for each type of analysis on a regular PC (CPU: $2.6 \mathrm{GHz}, \mathrm{RAM}: 8 \mathrm{~GB}$ ) is shown in Table 5.

A PCA-based model reduction scheme was proposed for calculation of the coupling sensitivities. To check the quality of the obtained sensitivities, Table 5.3 compares the sensitivity values obtained with and without using the proposed reduction scheme. 


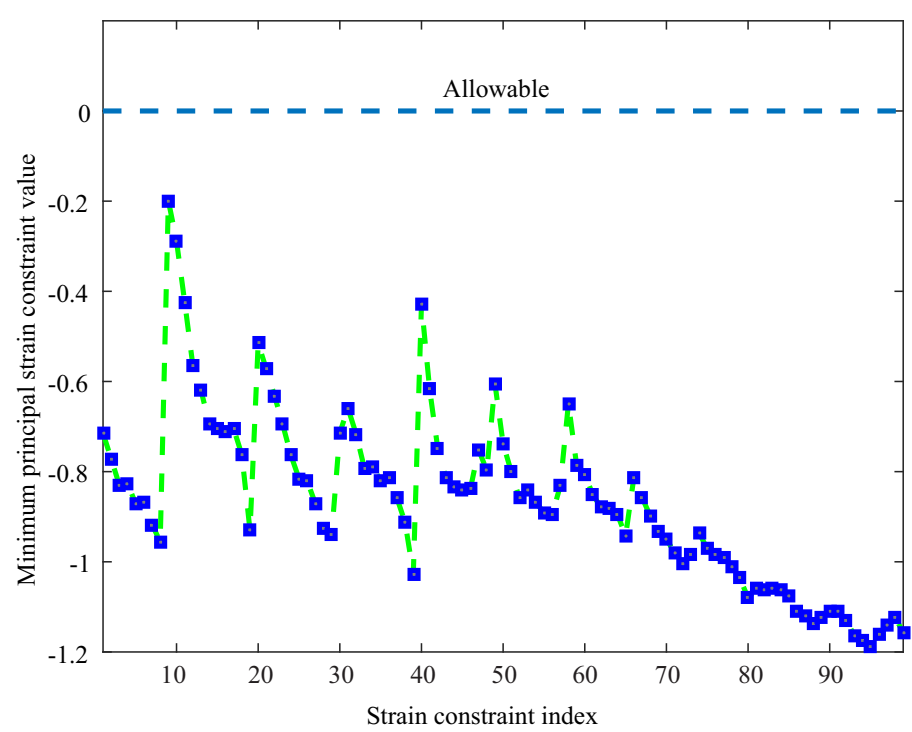

(a) The minimum principal strain value.

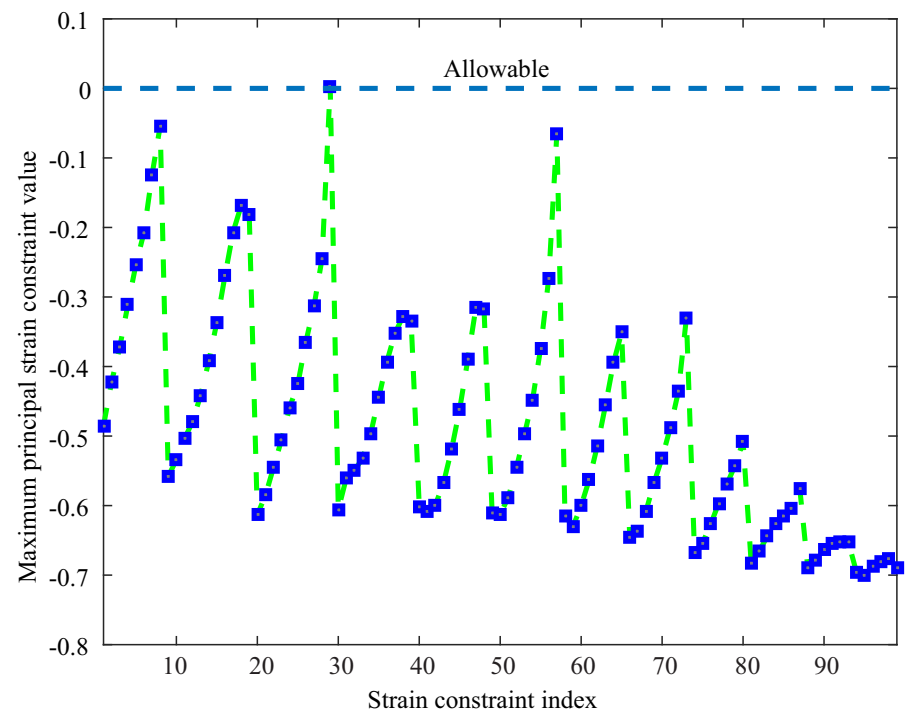

(b) The maximum principal strain value.

Figure 19: The value of the bottom skin strain constraint (for a central element) in each panel shown in Figure 5. 
Table 4: Detailed information about the number of iterations performed in the riblevel and wing-level optimization of each full iteration.

\begin{tabular}{cccccc}
\hline & & \multicolumn{4}{c}{ Full iteration } \\
Optimization level & Part & 1 & 2 & 3 & 4 \\
\hline Rib-level & Rib 2 & 20 & 18 & 4 & 1 \\
& Rib 7 & 23 & 16 & 5 & 2 \\
Wing-level & Rib 11 & 18 & 12 & 4 & 1 \\
& Top and bottom skin & 15 & 9 & 3 & 1 \\
\hline
\end{tabular}

Table 5: The (average) computation time required for each type of finite element analysis.

\begin{tabular}{lc}
\hline Analysis type & Time $(\mathrm{s})$ \\
\hline Rib buckling & 8 \\
Rib compliance & 6 \\
Skin buckling & 180 \\
Skin strain & 40 \\
Wing box bending & 50 \\
\hline
\end{tabular}

As seen in Table. 5.3, the sensitivity values with a relatively higher magnitude obtained using the PCA-based scheme, are in a good agreement with those obtained without using the proposed reduction scheme. According to Table. 5.3, the sensitivity of rib 2 active constraint to design variables $7,8,9,16,17$, and 18 is zero. The reason is that these design variables are located at the tip of the wing (see Figure 12) and their perturbed value has no effect on the loads applied to the rib which is located at the root of the wing. Table 7 shows the eigenvalues of the correlation matrix of the derivatives of the load vectors applied to rib $2\left(\partial \mathrm{L}_{\mathrm{rib} 2} / \partial \Phi_{\text {skin }}^{N}\right)$ as defined in Equation (13).

As seen in Table 7, the first 6 eigenvalues of the correlation matrix are (almost) zero, while the rest have considerable values. A reduced eigenvector matrix can be obtained by removing the eigenvectors corresponding to negligible eigenvalues. As suggested in the proposed model reduction scheme, the load derivative matrix $\left(\mathbf{L}_{\Phi}\right)$ can then be approximated by the reduced eigenvector matrix (see Equation (17)).

Using the proposed model reduction scheme, the number of finite element analyses related to the active constraint of the rib reduces from 18 to 12 (33\% reduction). These finite element analyses were required to calculate the skin-rib coupling terms and have to be calculated in every full iteration of the two-level optimization problem.

Note that if there were more design variables close to design variables $7,8,9,16$, 17, 18 (see Figure 12), a similar reduced space as obtained here could have been obtained. That is, in general, the percentage of reduction in the computation of coupling sensitivities may be increased when more design variables are assigned to each skin. 
Table 6: A comparison between the sensitivity values obtained with and without using the proposed model reduction scheme.

The sensitivity of the active compliance constraint of Rib 2 to skin design variables: $\partial \mathrm{g}_{\mathrm{rib} 2}^{*} / \partial \Phi_{\mathrm{skin}}^{N}$

\begin{tabular}{ccccccccccccccccccc}
\hline \multicolumn{11}{c}{ Design variable index (see Figure 12) } \\
\hline $\begin{array}{l}\text { design } \\
\text { variable } \\
\text { index }\end{array}$ & 1 & 2 & 3 & 4 & 5 & 6 & 7 & 8 & 9 & 10 & 11 & 12 & 13 & 14 & 15 & 16 & 17 & 18 \\
\hline no PCA & 0.39 & -12.08 & -2.37 & 0.86 & -3.19 & -0.37 & 0 & 0 & 0 & 0.95 & -0.24 & -1.36 & 0.67 & 2.72 & -1.95 & 0 & 0 & 0 \\
\hline PCA & -0.48 & -12.52 & -2.84 & 0.44 & -3.24 & -0.40 & 0 & 0 & 0 & 0.65 & -0.29 & -0.62 & -0.33 & 2.38 & -2.38 & 0 & 0 & 0 \\
\hline
\end{tabular}

The sensitivity of the active compliance constraint of Rib 7 to skin design variables: $\partial \mathrm{g}_{\text {rib7 }}^{*} / \partial \Phi_{\text {skin }}^{N}$

\begin{tabular}{|c|c|c|c|c|c|c|c|c|c|c|c|c|c|c|c|c|c|c|}
\hline & & & & & & Des & sign & variabl & le inde & x (see & Figur & re 12$)$ & & & & & & \\
\hline $\begin{array}{c}\text { design } \\
\text { variable } \\
\text { index }\end{array}$ & 1 & 2 & 3 & 4 & 5 & 6 & 7 & 8 & 9 & 10 & 11 & 12 & 13 & 14 & 15 & 16 & 17 & 18 \\
\hline no PCA & -0.27 & -3.59 & -0.79 & -14.36 & -44.90 & -22.43 & 1.51 & -5.51 & -2.31 & -1.74 & -6.92 & -6.15 & -0.43 & -26.82 & -6.38 & 1.35 & 3.61 & 0.42 \\
\hline PCA & 0.06 & -3.44 & -0.80 & -14.24 & -44.80 & -22.34 & 1.55 & -4.99 & -2.32 & -1.50 & -6.67 & -5.63 & -0.27 & -27.08 & -6.38 & 31.60 & 3.42 & 0.81 \\
\hline
\end{tabular}

The sensitivity of the active compliance constraint of Rib 11 to skin design variables: $\partial \mathrm{g}_{\text {rib11 }}^{*} / \partial \Phi_{\text {skin }}^{N}$

\begin{tabular}{|c|c|c|c|c|c|c|c|c|c|c|c|c|c|c|c|c|c|c|}
\hline \multicolumn{19}{|c|}{ Design variable index (see Figure 12) } \\
\hline $\begin{array}{l}\text { design } \\
\text { variable } \\
\text { index }\end{array}$ & 1 & 2 & 3 & 4 & 5 & 6 & 7 & 8 & 9 & 10 & 11 & 12 & 13 & 14 & 15 & 16 & 17 & 18 \\
\hline no PCA & -0.88 & -0.02 & 1.11 & -7.43 & -9.16 & -1.31 & 5.01 & -20.76 & -4.38 & -0.82 & -0.02 & -0.80 & 4.23 & -9.74 & -4.69 & 5.37 & -17.12 & -3.39 \\
\hline PCA & -0.88 & 0.05 & 1.14 & -7.39 & -9.55 & -1.25 & 4.86 & -21.49 & -4.34 & -0.82 & -0.01 & -0.74 & 4.15 & -9.75 & -4.66 & 5.36 & -17.24 & -3.32 \\
\hline
\end{tabular}


Table 7: The eigenvalues (in the ascending order) of the correlation matrix of the derivatives of the load vectors applied to rib $2\left(\partial \mathbf{L}_{\text {rib2 }} / \partial \Phi_{\text {skin }}^{N}\right)$ as defined in Eq (13).

\begin{tabular}{cccccc}
\hline index & eigenvalue & index & eigenvalue & index & eigenvalue \\
\hline 1 & $-4.1305 \mathrm{e}-10$ & 7 & $4.1690 \mathrm{e} 6$ & 13 & $3.3268 \mathrm{e} 7$ \\
2 & 0 & 8 & $5.6564 \mathrm{e} 6$ & 14 & $3.8184 \mathrm{e} 7$ \\
3 & 0 & 9 & $1.0833 \mathrm{e} 7$ & 15 & $6.2412 \mathrm{e} 7$ \\
4 & 0 & 10 & $1.2426 \mathrm{e} 7$ & 16 & $1.3125 \mathrm{e} 8$ \\
5 & $1.5933 \mathrm{e}-9$ & 11 & $1.6045 \mathrm{e} 7$ & 17 & $3.8062 \mathrm{e} 8$ \\
6 & $4.9036 \mathrm{e}-9$ & 12 & $2.2122 \mathrm{e} 7$ & 18 & $5.9194 \mathrm{e} 8$ \\
\hline
\end{tabular}

\section{Summary and conclusions}

A two-level procedure for the optimization of the stiffened composite skins and ribs of a wing box was presented. The optimization of the ribs is coupled with the optimization of the skins through the crushing loads $[14,15]$ applied to the ribs.

In the rib-level optimization, the crushing loads applied to the ribs are kept constant. The skin-rib coupling sensitivities are calculated at the end of a rib-level optimization. This coupling sensitivity describes the sensitivity of the optimized weight of the ribs to skins design variables. The skin-rib coupling sensitivities are used to calculate the gradient of the objective function in the wing-level optimization. In the wing-level optimization, the top and the bottom skins are also coupled through the loads applied to them.

A model reduction scheme based on the Principal Component Analysis (PCA) is introduced to reduce the number of finite element analyses required to calculate the coupling sensitivities.

The proposed method was applied to a wing box optimization problem where the weight of the top and the bottom skin as well as 3 ribs is the target for optimization. The results show that such a complex problem can be optimized at a reasonable computation cost using the proposed two-level approach.

It was shown that the proposed model reduction scheme provides coupling sensitivity information which is in good agreement with that obtained without such a scheme. In the presented example, the proposed model reduction scheme reduced the number of finite element analyzes related to one of the studied ribs (rib 2) active constraint by $33 \%$. This amount of reduction in the computations is gained in every full iteration of the optimization problem.

As discussed in Section 5, the weight of the ribs increases from the first towards the last iteration of the optimization. The reason is that the skins weight decreases throughout the optimization procedure and thus the crushing loads on the ribs increase. As the decrease in the skins weight outweighs the increase in the ribs weight, the overall weight of the wing box gradually decreases from the first towards the last iteration.

Only three ribs were considered for optimization in this work. Therefore, the skins' weight has a larger influence on the optimum design. If all ribs were considered 
for optimization, the design would have been biased towards the ribs' weight (see Equation 24).

Following the results, it can be concluded that for the presented example, the design of the wing-level optimization is governed by the maximum principal strain of the bottom skin as well as the crushing loads applied to the ribs.

The proposed method allows for the optimization of the ribs in parallel. Parallel computing may be used when all ribs are aimed to be optimized.

In the present study the effect of the ribs design on the skins design is neglected. This effect has to be considered in a future work.

The optimization of the number and the location of the spars, the material of the spars and the number and the location of the ribs is the subject for future research.

\section{Acknowledgements}

The support of this research by partners in TAPAS2 project is gratefully acknowledged. 


\section{References}

[1] R. A. Martins and A. B. Lambe. Multidisciplinary design optimization: A survey of architectures. AIAA Journal, 51(9):2049-2075, 2013.

[2] J. Sobieszczanski-Sobieski and R. T. Haftka. Multidisciplinary aerospace design optimization: Survey of recent developments. Structural Optimization, 14(1):123, 1997.

[3] Q. Liu, M. Jrad, S. B. Mulani, and R. K. Kapania. Global/local optimization of aircraft wing using parallel processing. AIAA Journal, 54(11):3338-3348, 2016.

[4] B. Liu, R. T. Haftka, and L. T. Watson. Global-local structural optimization using response surfaces of local optimization margins. Structural and Multidisciplinary Optimization, 27(5):352-359, 2004.

[5] L. A. Schmit and R. K. Ramanathan. Multilevel approach to minimum weight design including buckling constraints. AIAA Journal, 16(2):97-104, 1978.

[6] L. A. Schmit and M. Mehrinfar. Multilevel optimum design of structures with fiber-composite stiffened-panel components. AIA A Journal, 20(1):138-147, 1982.

[7] J. Sobieszczanski-Sobieski, B. B. James, and A. R. Dovi. Structural optimization by multilevel decomposition. AIAA Journal, 23(11):1775-1782, 1985.

[8] I. Sobieski and I. Kroo. Aircraft design using collaborative optimization. In 34th Aerospace Sciences Meeting and Exhibit, Aerospace Sciences Meetings, 1996.

[9] R. Braun, P. Gage, I. Kroo, and I. Sobieski. Implementation and performance issues in collaborative optimization. In 6th Symposium on Multidisciplinary Analysis and Optimization, Multidisciplinary Analysis Optimization Conferences, pages 295-305, 1996.

[10] J. Shankar, R. T. Haftka, and L. T. Watson. Computational study of a nonhierarchical decomposition algorithm. Computational Optimization and Applications, 2(3):273-293, 1993.

[11] N. M. Alexandrov and R. M. Lewis. Analytical and computational aspects of collaborative optimization for multidisciplinary design. AIAA Journal, 40(2):301309, 2002.

[12] R. T. Haftka and L. T. Watson. Decomposition theory for multidisciplinary design optimization problems with mixed integer quasiseparable subsystems. Optimization and Engineering, 7(2):135-149, 2006.

[13] R. T. Haftka and L. T. Watson. Multidisciplinary design optimization with quasiseparable subsystems. Optimization and Engineering, 6(1):9-20, Mar 2005.

[14] L. S. Cecchini and P. M. Weaver. Brazier effect in multibay airfoil sections. AIAA Journal, 43(10):2252-2258, 2005. 
[15] B. K. Stanford and P. D. Dunning. Optimal topology of aircraft rib and spar structures under aeroelastic loads. Journal of Aircraft, 52(4):1298-1311, 2015.

[16] J. S. Arora. Introduction to optimum design. Academic Press, Boston, third edition, 2012.

[17] F. Farzan Nasab, H. J. M. Geijselaers, I. Baran, R. Akkerman, and A. de Boer. A level-set-based strategy for thickness optimization of blended composite structures. Composite Structures, 206:903 - 920, 2018.

[18] I. Baran, C. C. Tutum, and J. H. Hattel. Optimization of the thermosetting pultrusion process by using hybrid and mixed integer genetic algorithms. Applied Composite Materials, 20(4):449-463, Aug 2013.

[19] C. C. Tutum, I. Baran, and K. Deb. Optimum design of pultrusion process via evolutionary multi-objective optimization. The International Journal of Advanced Manufacturing Technology, 72(9):1205-1217, Jun 2014.

[20] Y. M. Meddaikar, F. X. Irisarri, and M. M. Abdalla. Laminate optimization of blended composite structures using a modified shepard's method and stacking sequence tables. Structural and Multidisciplinary Optimization, 55(2):535-546, 2017.

[21] F. Farzan Nasab, G. A. Duipmans, H. J. M. Geijselaers, and A. de Boer. A gradient-based strategy for the optimization of stiffened composite structures subject to multiple load cases and multiple failure criteria. In EngOpt 2018 Proceedings of the 6th International Conference on Engineering Optimization, pages 1045-1054. Springer International Publishing, 2019.

[22] F. X. Irisarri, A. Lasseigne, F. H. Leroy, and R. Le Riche. Optimal design of laminated composite structures with ply drops using stacking sequence tables. Composite Structures, 107:559-569, 2014.

[23] F. Farzan Nasab, H. J. M. Geijselaers, I. Baran, and A. de Boer. Generating the best stacking sequence table for the design of blended composite structures. In Advances in Structural and Multidisciplinary Optimization: Proceedings of the 12th World Congress of Structural and Multidisciplinary Optimization (WCSMO12), pages 779-788, 2018.

[24] D. Liu, V. V. Toroporov, O. M. Querin, and D. C. Barton. Bilevel optimization of blended composite wing panels. Journal of Aircraft, 48(1):107-118, 2011. 



\section{Part III}

Appendices 



\section{Constrained steepest-descent method}

\section{Constrained steepest-descent algorithm}

Constrained steepest-descent (CSD) is a (close to) linear optimization algorithm for solving constrained optimization problems. One of the advantages of this method is that it is proved to converge to a local minimum starting from any point [1]. In the present research, the CSD algorithm is used to iteratively improve an initial design towards an optimize design. In the following the CSD algorithm is described in a step-by-step form:

1. Initialize the optimization variables. This includes the iteration number $k(=0)$, design variables vector $\mathbf{x}^{(0)}$, initial penalty parameter $R_{0}$ (typically 1 ), permissible constraint violation $\epsilon_{1}$, and convergence parameter $\epsilon_{2}$.

2. Calculate the value of the objective $(F)$ and constraint functions and their gradients at $\mathbf{x}^{(k)}$. Determine the maximum constraint violation $V_{k}=V\left(\mathbf{x}^{(k)}\right)$ through:

$$
V_{k}=\max \left\{0, g_{1}, g_{2}, \ldots, g_{m}\right\}
$$

where $g_{i}\left(\mathbf{x}^{(k)}\right)$ represents the constraints value at $\mathbf{x}^{(k)}$.

3. Use the values and the gradients of objective and constraint functions to form and solve a quadratic programming (QP) subproblem (discussed in 1.1). This yields a search direction $\mathbf{d}^{(k)}$ and the Lagrange multiplier vector $\boldsymbol{\mu}^{(k)}$.

4. Check for the stopping criteria $\left\|\mathbf{d}^{(k)}\right\| \leq \varepsilon_{2}$ and the maximum constraint violation $V_{k} \leq \varepsilon_{1}$. If these criteria are satisfied, stop. Otherwise, continue.

5. Update the penalty parameter $R_{k+1}$ (which is used for calculating the step size) through:

$$
R_{k+1}=\max \left\{R_{k}, r_{k}\right\} .
$$




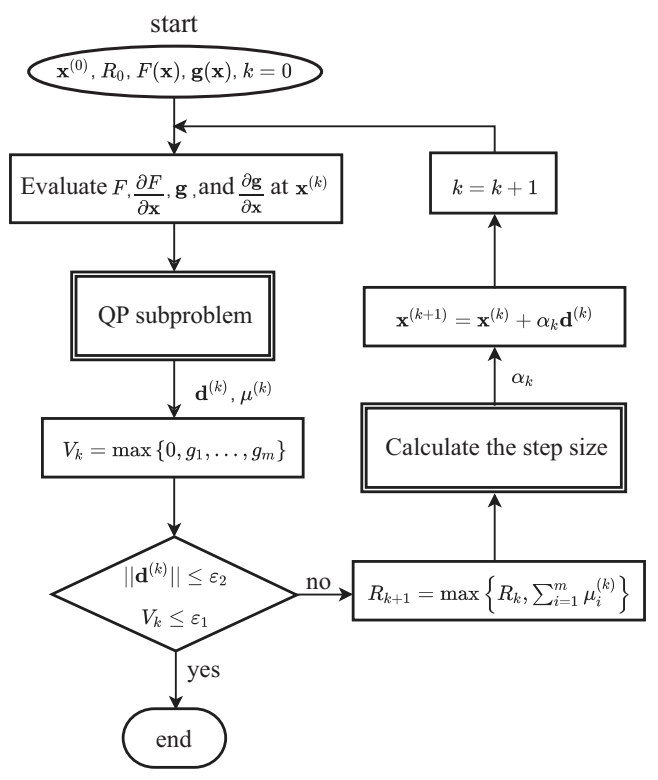

Figure 1: The flowchart of the CSD optimization algorithm.

where $r_{k}$ is the sum of the Lagrange multipliers:

$$
r_{k}=\sum_{i=1}^{m} \mu_{i}^{(k)}
$$

6. Obtain a proper step size $\alpha_{k}$ as explained in Section 1.2. Update the design through:

$$
\mathbf{x}^{(k+1)}=\mathbf{x}^{(k)}+\alpha_{k} \mathbf{d}^{(k)}
$$

7. Save the current penalty parameter and update the iteration counter as $k=$ $k+1$. Go to step 2 .

The flowchart of the CSD optimization algorithm is shown in Figure 1. 


\subsection{The Quadratic Programming subproblem}

The quadratic programming (QP) subproblem is used to determine the (descent) search direction and the Lagrange multipliers for the active constraints. The search direction together with a proper step size are used to update the design. The Lagrange multipliers are used in the procedure of finding a proper step size as will be explained in Section 1.2.

The QP subproblem is formed by linearizing the objective and the constraint functions. The linearized subproblem can be bounded if the length of the search direction $\|\mathbf{d}\|$ is required to be minimized in addition to the minimization of the linearized objective function. This is achieved by combining the two objectives. The objective function of the QP subproblem is defined as:

$$
\min \quad \bar{f}=\mathbf{c}^{T} \mathbf{d}+\frac{1}{2} \mathbf{d}^{T} \mathbf{d}
$$

where $\bar{f}$ represents the objective function of the QP subproblem, and $\mathbf{c}$ represents the gradient of the objective function at the current design: $c_{i}=\frac{\partial F\left(\mathbf{x}^{(k)}\right)}{\partial x_{i}}$.

The constraint of the QP subproblem is defined as:

$$
\mathbf{A d} \leq \mathbf{b}
$$

where $\mathbf{A}$ represents the gradients of the inequality constraints at the current design: $a_{j i}=\frac{\partial g_{j}\left(\mathbf{x}^{(k)}\right)}{\partial x_{i}}$, and $\mathbf{b}$ represents the negatives of the inequality constraint function values.

Substantial research effort has been dedicated to solving the QP subproblem efficiently. If the problem is simple, the KKT conditions of optimality (see [1]) can be used to solve the problem. The Simplex method [1] (to solve a linear programming problem) is extended to solve a general QP subproblem. The details can be found in $[1]$.

\subsection{Step size calculation}

A proper step size along the search direction has to be calculated. This is achieved by minimizing a descent function along the search direction. Here, the Pshenichny's descent function [1] is used. This descent function is defined as:

$$
\Theta_{k}=F\left(\mathbf{x}^{(k)}\right)+R_{k} V_{k}
$$

where $\Theta$ is the Pshenichny's descent function, $F$ is the objective function of the original problem, $R$ is the penalty parameter, and $V$ is the maximum constraint violation.

The descent function can be minimized using inexact line search. Following this method, different step size values are tried until the condition of sufficient reduction in the descent function (defined in Equation 10) is satisfied. 
The trial step size $\alpha_{j}$ for (sub)iteration $j$ is given by:

$$
\alpha_{j}=(\delta)^{j}, \quad j=0,1,2, \ldots,
$$

where $\alpha_{j}$ is the $j^{\text {th }}$ trial step size. $\delta$ is constant which is typically 0.5 . The trial design is then computed using:

$$
\mathbf{x}^{(k+1, j)}=\mathbf{x}^{(k)}+\alpha_{j} \mathbf{d}^{(k)}
$$

where $\mathbf{x}^{(k+1, j)}$ is the $j^{\text {th }}$ trial design at iteration $k$.

A trial step size is accepted if the following descent condition is satisfied:

$$
\Theta_{k+1, j} \leq \Theta_{k}-\alpha_{j} \gamma\left\|\mathbf{d}^{(k)}\right\|^{2}
$$

where $\Theta_{k+1, j}$ is defined as:

$$
\Theta_{k+1, j}=\Theta\left(x^{(k+1, j)}\right)=F_{k+1, j}+R V_{k+1, j} .
$$

and $\gamma$ is a specified constant between 0 and 1 .

\section{References}

[1] J. S. Arora. Introduction to optimum design. Academic Press, Boston, third edition, 2012. 


\section{Horizontal stabilizer skin optimization}

One target of the present research is to optimize the skin of a Horizontal Stabilizer (HS) of an aircraft. Unlike a common HS that is composed of two separate left and right sides, the structure concerned here is a single part as shown in Figure 1. The aim is to manufacture this structure as a single part to enhance the strength of the HS.

The $0^{\circ}$ axis of the local coordinate system on either side of the HS is aligned with the rear spar of that side. The local coordinate systems and the sweep angle of the HS are shown schematically in Figure 2.

Because of the sweep angle of the HS, the plies with standard orientations $\left(0^{\circ}, \pm 45^{\circ}\right.$, and $90^{\circ}$ ) of fiber angles with respect to the local coordinate system on either side of the HS appear to have non-standard orientations with respect to the non-local coordinate system. For example, a ply with $0^{\circ}$ of fiber orientation on the right side of the HS, gets $50^{\circ}$ fiber orientation with respect to the left side coordinates system, when the fibers are extended from the right side to the left side of the HS. Table 1 shows the standard fiber orientations and the orientations the fibers obtain when they are looked at from a non-local coordinate system (mutual orientations).

Table 1: Standard fiber orientations and the orientations they obtain when they are looked at from a non-local coordinate system.

\begin{tabular}{cc}
\hline $\begin{array}{c}\text { Standard orientation } \\
\text { (local coordinate system) }\end{array}$ & $\begin{array}{c}\text { Orientations with respect to the } \\
\text { non-local coordinate system }\end{array}$ \\
\hline $0^{\circ}$ & $50^{\circ}$ \\
$45^{\circ}$ & $5^{\circ}$ \\
$90^{\circ}$ & $-40^{\circ}$ \\
$-45^{\circ}$ & $-85^{\circ}$ \\
\hline
\end{tabular}




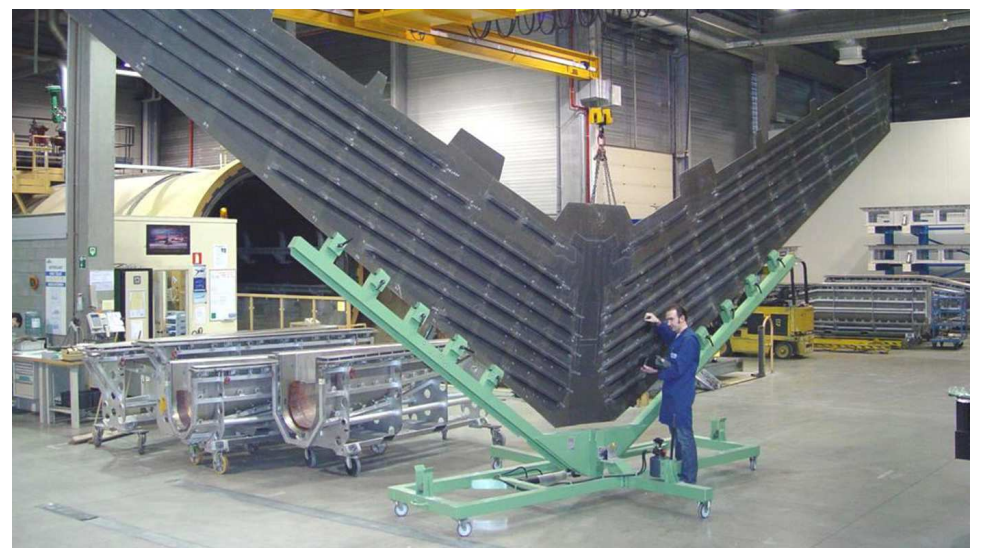

Figure 1: The horizontal stabilizer of an aircraft as a single part. Google image.

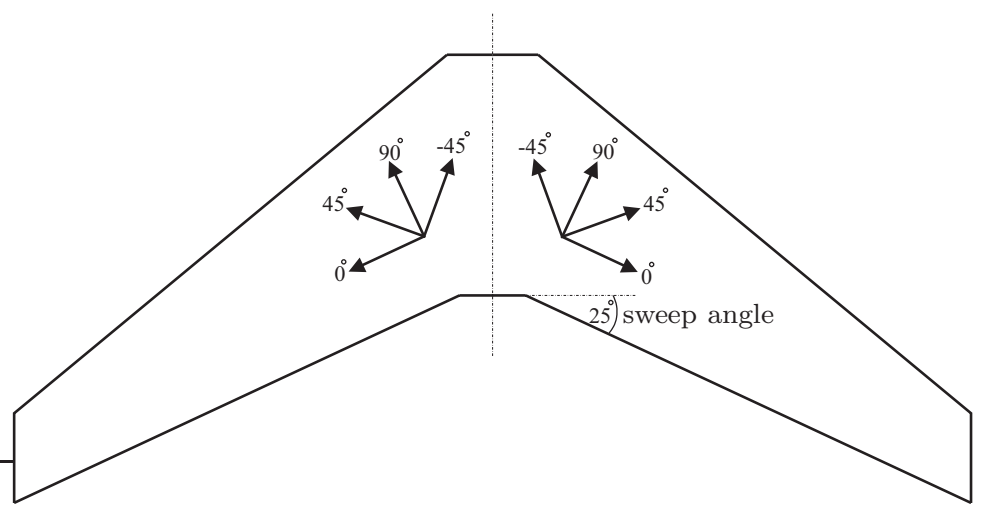

Figure 2: The local coordinate systems and the sweep angle of the horizontal stabilizer. 
Here, to fulfill the design guidelines (such as balance, disorientation, percentage rule, and contiguity), the non-standard orientations are approximated with the closest standard orientations, i.e.: $50^{\circ} \approx 45^{\circ}, 5^{\circ} \approx 0^{\circ},-40^{\circ} \approx-45^{\circ}$, and $-85^{\circ} \approx 90^{\circ}$

An SST has to be generated for laminates on both the left and the right side of the HS. Figure 3 shows this SST. The laminates with the same thickness on either side of the HS have to have (close to) similar stacking sequences. Moreover, every two laminates with the same thickness from either side of the SST have to be continuous (blended) with respect to each other. This requires further considerations when dropping plies. As an example, Figure 4 shows two laminates with the same thickness value from both sides, placed next to each other. As seen in Figure 4, in each row either the orientations are the same or they are mutual orientations. This means that if these two laminates are placed on the left and the right side of the HS, plies are continuous. Likewise, the continuity (blending) between the left and the right side of the HS is guaranteed when any arbitrary laminate is prescribed by the optimizer from the SST shown in Figure 3.

As a part of the present $\mathrm{PhD}$ research, a program was developed to generate SSTs for both the left and the right side of the HS. Fulfilling the design and the manufacturability guidelines as well as the left-right symmetric laminates (specifically for the HS problem), results in a large number of valid SSTs (thousands of SSTs when the thickest laminate has 64 plies). The method proposed in Paper A must be employed to generate the optimized SST with respect to the problem. 
left side

right side

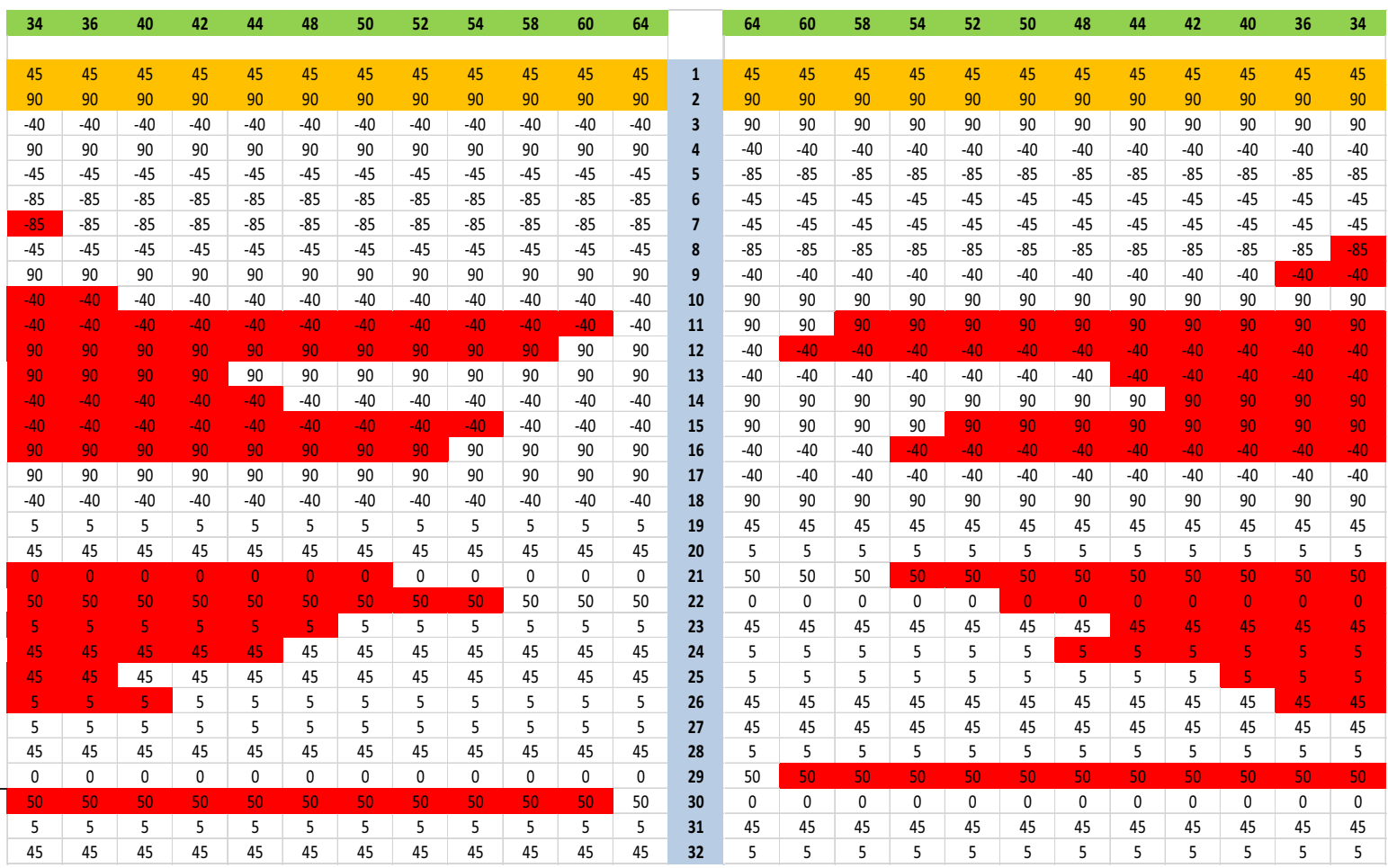

Figure 3: An SST for the left and the right side of the horizontal stabilizer. The first two plies in each laminate are the covering plies and the red color indicates dropped plies. 


\begin{tabular}{|c|c|}
\hline 34 & 34 \\
\hline 45 & 45 \\
\hline 90 & 90 \\
\hline-40 & 90 \\
\hline 90 & -40 \\
\hline-45 & -85 \\
\hline-85 & -45 \\
\hline-45 & -45 \\
\hline 90 & 90 \\
\hline 90 & -40 \\
\hline-40 & 90 \\
\hline 5 & 45 \\
\hline 45 & 5 \\
\hline 5 & 45 \\
\hline 45 & 5 \\
\hline 0 & 0 \\
\hline 5 & 45 \\
\hline 45 & 5 \\
\hline
\end{tabular}

Figure 4: Every two laminates with the same thickness from either side of the SST have to be continuous (blended) with respect to each other. Here, laminates with 34 plies are shown. 



\section{Nomenclature}

\section{Abbreviations}

$\begin{array}{ll}\text { AFP } & \text { Automated Fiber Placement } \\ \text { ATL } & \text { Automated Tape Laying } \\ \text { CSD } & \text { Constrained steepest-descent } \\ \text { DMO } & \text { Discrete Material Optimization } \\ \text { DMTO } & \text { Discrete Material and Thickness Optimization } \\ \text { GA } & \text { Genetic Algorithm } \\ \text { GFRP } & \text { Glass Fiber Reinforced Polymer } \\ \text { HS } & \text { Horizontal Stabilizer } \\ \text { KKT } & \text { Karush-Kuhn-Tucker } \\ \text { LSF } & \text { Level-set function } \\ \text { PCA } & \text { Principal Component Analysis } \\ \text { QP } & \text { Quadratic Programming } \\ \text { RAMP } & \text { Rational Approximation of Material Properties } \\ \text { RMSE } & \text { Root Mean Square Error } \\ \text { SIMP } & \text { Solid Isotropic Material with Penalization } \\ \text { SQP } & \text { Sequential Quadratic Programming } \\ \text { SST } & \text { Stacking Sequence Table } \\ \text { TAPAS } & \text { Thermoplastic Affordable Primary Aircraft Structures }\end{array}$




\section{Roman symbol}

A Extensional stiffness matrix

B Bending-Extension coupling matrix

C Correlation matrix

C Compliance of the structure

D Bending stiffness matrix

d Search direction vector

$f \quad$ Objective function

$g \quad$ Constraint function

I Identity matrix

K Structural stiffness matrix

$\mathbf{K}_{\mathrm{B}} \quad$ Structural bending stiffness matrix

$\mathbf{K}_{\mathrm{G}} \quad$ Structural stress stiffness matrix

L Load vector

$t \quad$ Ply thickness

V Eigenvector matrix

$V \quad$ Maximum constraint violation

$W \quad$ Structural weight

$w_{i} \quad$ The $i^{\text {th }}$ eigenvector of an eigenvalue problem

$X \quad$ Spatial coordinate

\section{Greek symbol}

$\begin{array}{ll}\alpha & \text { Trial step size } \\ \Gamma & \text { Interpolation function } \\ \varepsilon_{\mathrm{C}} & \text { Allowable compressive strain } \\ \varepsilon_{\max } & \text { Maximum principal strain } \\ \varepsilon_{\min } & \text { Minimum principal strain } \\ \varepsilon_{\mathrm{T}} & \text { Allowable tensile strain } \\ \Theta & \text { Pshenichny's descent function } \\ \boldsymbol{\Lambda} & \text { Eigenvalue matrix } \\ \lambda_{i} & \text { The } i^{\text {th }} \text { eigenvalue of a problem } \\ \boldsymbol{\mu} & \text { Lagrange multipliers vector } \\ \rho & \text { Ply density } \\ \Phi & \text { Level-set function } \\ \boldsymbol{\Psi} & \text { Principal components basis } \\ \Omega & \text { Structural domain }\end{array}$




\section{Publications}

\section{Journal papers}

1. F. Farzan Nasab, H. J. M. Geijselaers, I. Baran, R. Akkerman, and A. de Boer. A levelset-based strategy for thickness optimization of blended composite structures. Composite Structures, 206:903 - 920, 2018.

2. F. Farzan Nasab, H. J. M. Geijselaers, I. Baran, R. Akkerman, and A. de Boer. Topology, shape, and thickness optimization of composite structures considering design, manufacturing, and strength related guidelines. Submitted to Structural and Multidisciplinary Optimization. February 2019.

3. F. Farzan Nasab, H. J. M. Geijselaers, I. Baran, R. Akkerman, and A. de Boer. Optimization of the interacting stiffened skins and ribs made of composite materials. Submitted to AIAA Journal. February 2019.

\section{Conference papers}

1. F. Farzan Nasab, H. J. M. Geijselaers, and A. de Boer. A multi-level set gradient based algorithm for buckling optimization of blended composite structures. 5th Aircraft Structural Design Conference. Royal Aeronautical Society, Manchester, 2016.

2. F. Farzan Nasab, H. J. M. Geijselaers, I. Baran, and A. de Boer. Generating the best stacking sequence table for the design of blended composite structures. In Advances in Structural and Multidisciplinary Optimization: Proceedings of the 12th World Congress of Structural and Multidisciplinary Optimization (WCSMO12), pages 779-788, 2018.

3. F. Farzan Nasab, G. A. Duipmans, H. J. M. Geijselaers, and A. de Boer. A gradient-based strategy for the optimization of stiffened composite structures subject to multiple load cases and multiple failure criteria. In EngOpt 2018 Proceedings of the 6th International Conference on Engineering Optimization, pages 1045-1054. Springer International Publishing, 2019. 


\section{Supervision}

1. Optimization of a horizontal tailplane made of blended composites. G.A. Duipmans, MSc thesis, University of Twente, 2018.

2. Semi-analytical buckling sensitivity calculation for the optimization of composite structures. W. Korbee, MSc thesis, University of Twente, 2018.

3. Evolutionary algorithm-based optimization of multi-panel composite structures. B. Nijhuis, Capita Selecta, Applied Mechanics, University of Twente, 2018. 


\section{Acknowledgements}

I would like to acknowledge all the people who either directly helped me with my $\mathrm{PhD}$ project or through the years shaped my life so that I ended up doing a $\mathrm{PhD}$.

Knowledge and creativity are the two essentials to successfully finish a $\mathrm{PhD}$ project. In this four-year challenging journey, it is a sense of relief when there is a companion who can always add to what you know and you can always trust what he says. A guide who can shape your creativity towards the right path is highly valued. With this introduction, I would like to gratefully acknowledge the care and the support of my supervisor Bert Geijselaers throughout my PhD project. Bert, in the past few years I learned a lot from you. This project could have not been as smooth as it proceeded without your support. Many thanks for that.

I would like to express my deepest appreciation to the advisors of my $\mathrm{PhD}$ project André de Boer, Remko Akkerman, and Ismet Baran. Your valuable support throughout this research period was a significant help towards achieving the project goals. Many thanks to Ton van den Boogaard for providing me with the opportunity to attend DIEKA meetings and discuss my research progress there. I would like to thank Semih Perdahcioğlu and Javad Hazrati for their supports and comments during the DIEKA meetings.

I am extremely grateful to the members of the graduation committee Prof. dr. C. Bisagni, Prof. dr. ing. A. Schumacher, Prof. dr. ir. D.M. Brouwer, Prof. dr. I. Gibson for reading my thesis and showing interest in my work.

I kindly acknowledge the support of partners of TAPAS project. I would like to specially thank Pieter Lantermans, Ton van der Laan, and Arnt Offringa for their valuable technical support and providing FE models required for my work.

I very much appreciate the care and the support of Debbie Zimmerman van Woesik in the past four years. Dear Debbie, thank you for being so kind and supportive. Thanks should also go to Nico van Vliet and Axel Lok for their helps on preparing software and server. Many thanks to all my colleagues in Applied Mechanics for providing me with unforgettable memories and special thanks to my officemates Neda, Erkan, and Annemieke. I really enjoyed my time next to you in the past years. Ali and Mieke, thank you for helping me specially upon my arrival to the Netherlands. Jurnan, you added so much fun to my PhD life through all the amusement parks, Avatarz, and 
cooking events. Thanks for your company during the ladies nights! Boukje, thank you for motivating us to join and enjoy your Bootcamp sessions. This is what I will definitely miss in the future. Antonella, thank you for all nice Italian foods and cookies. I hope we arrange more events in the future. Thank you Frans for translating my thesis summary to Dutch. I will miss the lunch times we had together. Sikander, I really enjoyed all those spicy Chicken Biryanies and thanks for bringing mangoes all the way from Pakistan. It is an honor to grow muscles next to you. Omid, thank you for arranging BBQs and football events. Laura, the Highland games event was an amazing experience. Thank you for arranging it. Melissa, I think if some posters are replaced with your paintings, the corridor becomes a more attractive place. Thanks for your care about the cover design of my thesis. Jos, thank you for helping me with my MATLAB questions and also helping me with all the Dutch letters at the time that I could not read Dutch. I would like to thank my paranymphs Frans and Jurnan for supporting me during my defense session. Thanks also go to www.freepik.com for providing the background of this book cover.

I would like to thank the MSc students for their interest to do their final assignments and a course assignment in the direction of my project: Gerard Duipmans, Wouter Korbee, and Björn Nijhuis.

I would also like to extend my gratitude to our friends Mitra and Hassan. Thank you so much for all the invitations and all the super delicious foods you made. I am looking forward to organizing a lot of legendary events and trips next to you lovely people.

A special thanks to my friend Reza Jafari. It has been 14 years that I know you. We went to the same universities and we worked together. We shared our moments of worries and happiness. I wish our friendship stays on forever. I wish you and Mina a very happy and successful life.

I am extremely grateful to my parents Majid and Giti for their countless supports. Thank you for always motivating me to achieve high goals and standing next to me in all difficulties. I specially thank my sister Mahshad for her moral supports. Mahshad, I am proud of you and I encourage to be ambitious for highest goals. I am also very grateful to my uncle Bahram and my grand mother Akram for their moral supports in all past years.

I would like to express my deepest gratitude to my beloved wife Farnaz. During my work as a PhD, I was blessed to be your colleague besides being your life partner. You were next to me in the moments of disappointment and supported me morally. Every day we cycled together, we attended Dutch course together and we did sports together. I enjoyed every single moment of my past years next to you. You helped a lot with my thesis arrangements in latex despite being busy with your own work. I am deeply grateful to all theses. Thank you for treating me with patience and love.

Farshad Farzan Nasab, February 2019 

